This item was submitted to Loughborough's Research Repository by the author.

Items in Figshare are protected by copyright, with all rights reserved, unless otherwise indicated.

\title{
Extraordinary magnetoresistance in hybrid semiconductor-metal systems
}

PLEASE CITE THE PUBLISHED VERSION

PUBLISHER

(C) Thomas Hewett

LICENCE

CC BY-NC-ND 4.0

\section{REPOSITORY RECORD}

Hewett, Thomas H.. 2019. "Extraordinary Magnetoresistance in Hybrid Semiconductor-metal Systems". figshare. https://hdl.handle.net/2134/10231. 
This item was submitted to Loughborough's Institutional Repository (https://dspace.lboro.ac.uk/) by the author and is made available under the following Creative Commons Licence conditions.

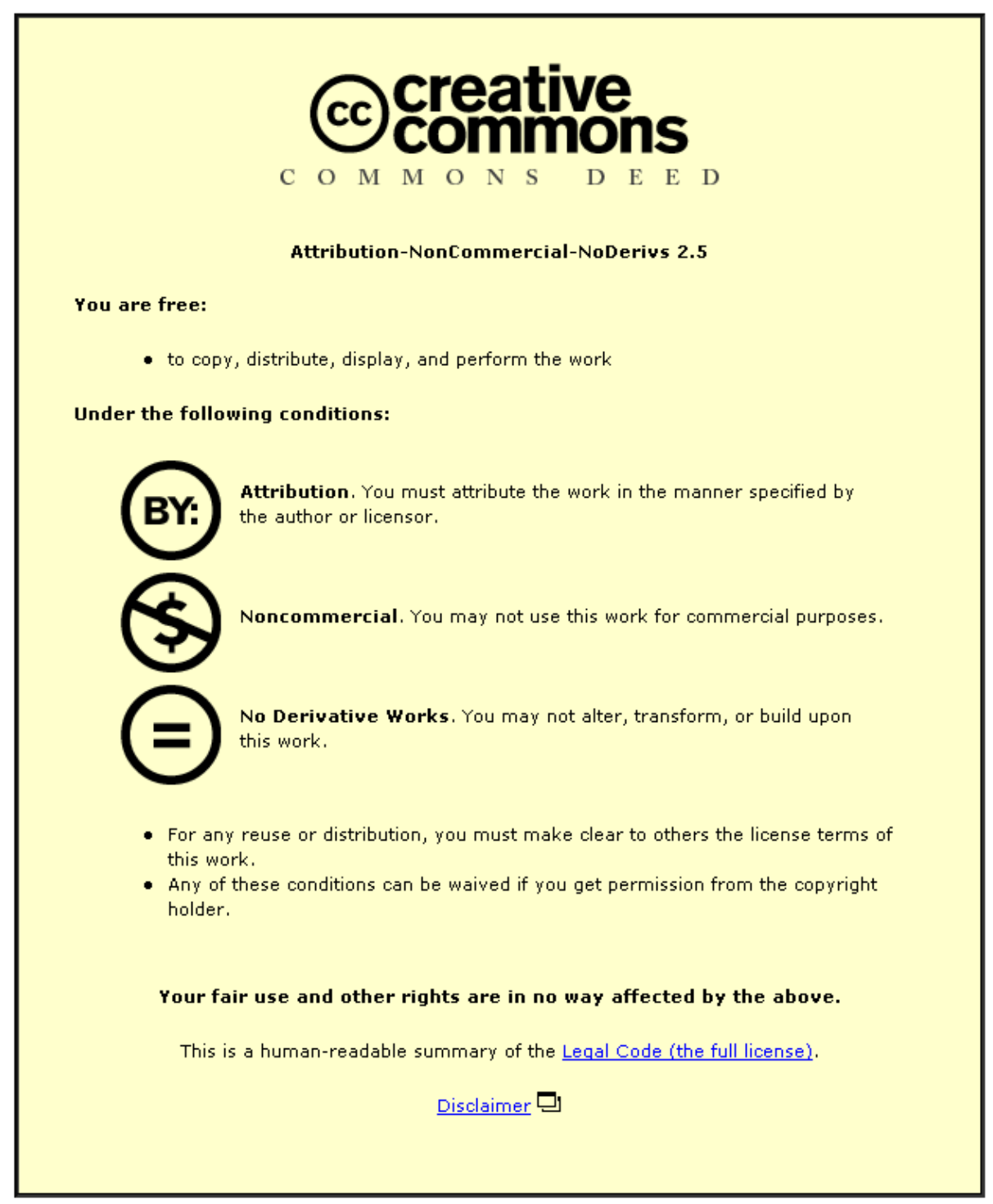

For the full text of this licence, please go to: http://creativecommons.org/licenses/by-nc-nd/2.5/ 


\title{
Extraordinary Magnetoresistance in Hybrid Semiconductor-Metal Systems
}

\author{
by \\ Thomas Hewett \\ A Doctoral Thesis \\ submitted in partial fulfilment of the requirements for the award \\ of \\ Doctor of Philosophy \\ in the \\ Faculty of Science \\ Department of Physics
}

August 2012

(C) by Thomas Hewett (2012) 


\section{Publications}

- T. H. Hewett and F. V. Kusmartsev. Extraordinary Magnetoresistance In Hybrid Semiconductor-Metal Systems. International Journal of Modern Physics B, 23(2021):4158, August 2009. Accessible via http://arxiv.org/abs/1007.5452.

- T. H. Hewett and F. V. Kusmartsev. Geometrically Enhanced Extraordinary Magnetoresistance In Semiconductor-Metal Hybrids. Physical Review B, 82(21):212404, December 2010.

- T. H. Hewett and F. V. Kusmartsev. Extraordinary Magnetoresistance: Sensing The Future. Central European Journal of Physics, DOI: 10.2478/s11534-012-0015-1, February 2012.

\section{Oral Presentations}

- TeraMat09, Benasque, Spain, December 2009.

- Physics Seminar, Loughborough University, UK, June 2010.

- UK Semiconductors 2010, Sheffield, UK, July 2010.

- Invited Seminar, The University of Nottingham, UK, September 2010.

- SigmaPhi 2011, Larnaca, Cyprus, July 2011.

- Materials Research Workshop, Loughborough University, UK, November 2011.

\section{Poster Presentations}

- TeraMat09, Benasque, Spain, December 2009.

- Graduate School Poster Competition, Loughborough University, UK, May 2010.

- Physics by the Lake Summer School, University of Cumbria, UK, July 2010.

- Science Poster Competition, Loughborough University, UK, April 2011.

(Awarded Commendation Prize)

- NES 2011, Bath, UK, May 2011.

- SigmaPhi 2011, Larnaca, Cyprus, July 2011. 


\section{Acknowledgements}

This Thesis would not have been possible without the support of my supervisor Prof. Feo Kusmartsev. Both from an academic and personal point of view, he has never failed to offer his expert guidance. I will never forget my first 'conference'; thanks for the skiing lessons!

I cannot put into words how much my parents and sister have helped me, without their unwavering support I would not have been able to reach this point. I sincerely appreciate everything you have done for me, thank you.

Loughborough Physics department has been my home away from home for the past six years. I would like to thank everyone in the department for their kindness especially Dr John Samson, Maureen McKenzie and Victoria Webster.

I would also like to thank numerous conference participants for their comments and insightful conversations that have ultimately guided my research, especially Prof. Bryan Gallagher who made me feel very welcome when invited for a discussion at the University of Nottingham.

Finally, I would like to thank all of the friends I have made here over the years. Without you I would not have made it; you know who you are.

"We choose to go to the moon in this decade and do the other things, not because they are easy, but because they are hard, because that goal will serve to organize and measure the best of our energies and skills, because that challenge is one that we are willing to accept, one we are unwilling to postpone, and one we intend to win."

John F. Kennedy.

"I think it was John Lennon who said: "Life is what happens when you're making other plans", and that's how I feel. Although he also said: "I am the Walrus I am the eggman" so I don't know what to believe."

Tim, The Office. 


\section{LOUGHBOROUGH UNIVERSITY}

\section{Abstract}

Faculty of Science

Department of Physics

Doctor of Philosophy

by Thomas Hewett

Systems that exhibit the extraordinary magnetoresistance (EMR) effect and other more disordered semiconductor-metal hybrid structures have been investigated numerically with the use of the finite element method (FEM). Initially, modelling focused on circular geometry EMR devices where a single metallic droplet is embedded concentrically into a larger semiconducting disk. The dependence of the magnetoresistance of such systems on the transverse magnetic field $(0-5 \mathrm{~T})$ and filling factor $(1 / 16-15 / 16)$ are reported and generally show a very good agreement with existing experimental data. The influence of the geometry of the conducting region of these EMR systems was then investigated. The EMR effect was found to be highly sensitive to the shape of the conducting region with a multi-branched geometry producing a four order of magnitude enhancement of the magnetoresistance over a circular geometry device of the same filling factor. Conformal mapping has previously been shown to transform a circular EMR device into an equivalent linear geometry. Such a linear EMR device has been modelled with the EMR mechanism clearly observed. The magnetoresistive response of a circular EMR device upon changes to: the mobility of the semiconducting region; the ratio of metal to semiconductor conductivity; and the introduction of a finite resistance at the semiconductor-metal interface, have also been investigated. In order for a large EMR effect to be observed the system requires: the semiconductor mobility to be large; the conductivity of the metal to be greater than two orders of magnitude larger than that of the semiconductor; and a very low interface resistance. This modelling procedure has been extended to include inhomogeneous semiconductor-metal hybrids with a more complex and disordered structure. Two models are presented, both based upon the random distribution of a small proportion of metal inside a semiconducting material. The resultant magnetoresistance in each case is found to have a quasi-linear dependence on magnetic field, similar to that observed in the silver chalcogenides. 


\section{Contents}

Publications i

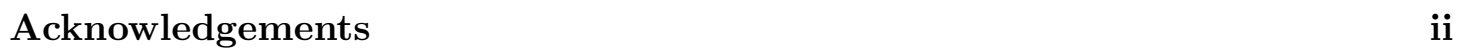

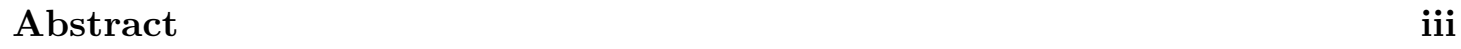

Contents iv

List of Figures vi vi vis

List of Tables $\quad$ xvi

$\begin{array}{lll}1 & \text { Introduction } & 1\end{array}$

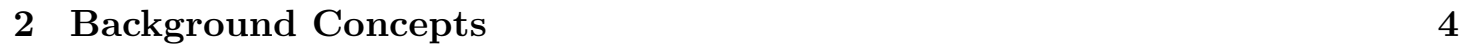

$2.1 \quad$ Magnetoresistance . . . . . . . . . . . . . . . . . . . . 4

2.1 .1 Ordinary Magnetoresistance (OMR) . . . . . . . . . . . . . . . . . . . . 4

2.1 .2 Anisotropic Magnetoresistance (AMR) $\ldots \ldots \ldots$. . . . . . . . . . 6

$2.1 .3 \quad$ Giant Magnetoresistance (GMR) $\ldots \ldots \ldots$. . . . . . . . . . . . . . . . 7

2.1.4 Tunnelling Magnetoresistance (TMR) . . . . . . . . . . . . . . . . . 9

2.1 .5 Colossal Magnetoresistance (CMR) . . . . . . . . . . . . . . . . . . . . . . . . . . . . 10

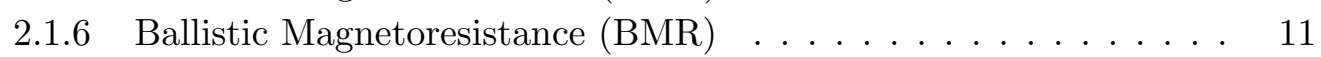

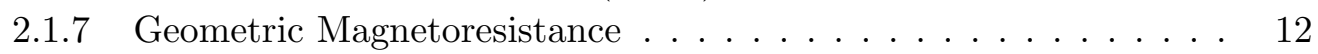

$2.1 .8 \quad$ Extraordinary Magnetoresistance (EMR) . . . . . . . . . . . . . . . 13

2.2 Hall Effect . . . . . . . . . . . . . . . . . . . . . . . . . . 15

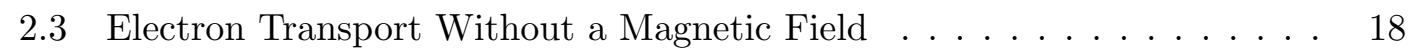

2.4 Electron Transport With a Magnetic Field . . . . . . . . . . . . . . . . . . . . . . . 20

$2.5 \quad$ Finite Element Method and Modelling . . . . . . . . . . . . . . . . . . 22

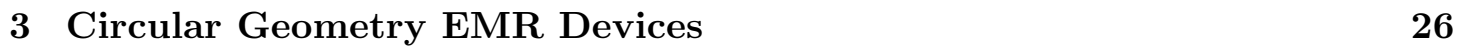

$3.1 \quad$ Previous Results in a Circular System Geometry . . . . . . . . . . . . . . . 26

$3.1 .1 \quad$ Experimental Data . . . . . . . . . . . . . . . . . . 26

$3.1 .2 \quad$ FEM Modelling . . . . . . . . . . . . . . . . . . . . . . . 35

3.2 Modelling Procedure . . . . . . . . . . . . . . . . . . . . . . . . . . . . . . 37

3.3 Modelling in a Circular System Geometry . . . . . . . . . . . . . . . . . . 39 
\begin{tabular}{|ll|}
4 & Influence of System Geometry \\
\hline
\end{tabular}

4.1 Previous Geometrical Results . . . . . . . . . . . . . . . . . . . 55

4.2 Geometrically Enhanced Magnetoresistance . . . . . . . . . . . . . . . . . 62

4.3 Linear EMR Device Geometry . . . . . . . . . . . . . . . . . . . . . . . . 72

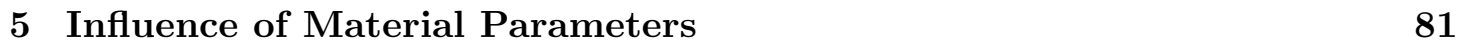

5.1 Previous Investigations of Material Parameters . . . . . . . . . . . . . . . . 81

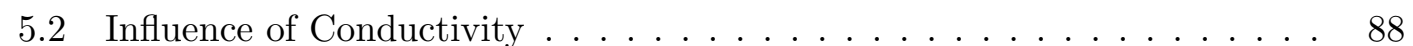

$5.3 \quad$ Influence of Mobility . . . . . . . . . . . . . . . . . . . . . 90

5.4 Influence of Interface Resistance . . . . . . . . . . . . . . . . . . . . . 93

$\begin{array}{lll}6 & \text { Magnetoresistance in Disordered Systems } & 99\end{array}$

6.1 Magnetoresistance in the Silver Chalcogenides . . . . . . . . . . . . . . . . 99

$6.1 .1 \quad$ Experimental Data . . . . . . . . . . . . . . . . . . . . . . 99

6.1 .2 Previous Theoretical Approaches . . . . . . . . . . . . . . . . . . . 102

6.2 Modelling in a Disordered System Geometry . . . . . . . . . . . . . . . . 104

\begin{tabular}{lll}
\hline 7 & Conclusion & 122
\end{tabular}

\begin{tabular}{lr}
\hline Appendices & 125
\end{tabular}

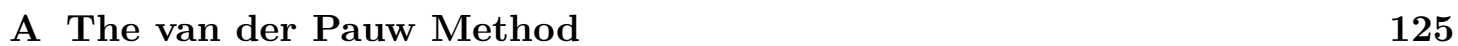

\begin{tabular}{lr}
\hline B Conformal Mapping & 129
\end{tabular}

\begin{tabular}{ll}
\hline C Research Poster & 135
\end{tabular}

\begin{tabular}{ll}
\hline D Comsol Multiphysics Code & 137
\end{tabular}

\begin{tabular}{ll}
\hline Bibliography & 141
\end{tabular} 


\section{List of Figures}

2.1 Magnetoresistance of copper at room temperature (297K) as a function of magnetic field (given in kilogauss, $1 \mathrm{~T}=10$ kilogauss). The experimental points are represented by the open circles while the solid line is a fitted function. The magnetoresistance reaches approximately $0.25 \%$ in a magnetic field of 10T. (Reproduced from reference 1). . . . . . . . . . 5

2.2 Representation of the anisotropic magnetoresistance in Permalloy, showing the difference in resistivity when the current is aligned perpendicular and parallel to the magnetic field. (Reproduced from reference 2). . . . . . . . 6

2.3 Illustration of the change in scattering cross-section when the current is aligned parallel with (high resistance) and perpendicular to (low resistance) the magnetic field. Here, the green ovals represent the scattering crosssection of bound electron orbits around each nucleus of the crystal lattice. 7

2.4 The initial discovery of GMR produced a maximum magnetoresistance value of approximately $50 \%$ at $4.2 \mathrm{~K}$ in a magnetic field of $2 \mathrm{~T}$. Here, a chromium (Cr) layer of varying thickness is positioned between layers of iron $(\mathrm{Fe})$. (Reproduced from reference 3 ). . . . . . . . . . . . 8

2.5 In GMR materials, applying a magnetic field changes the magnetisation of adjacent ferromagnetic (FM) layers from antiparallel to parallel. This results in different scattering processes of conduction electrons and thus a change in resistance $\left.{ }^{4}\right] \ldots \ldots \ldots \ldots \ldots$

2.6 Illustration showing tunnelling between two ferromagnetic (FM) layers separated by a thin insulating (I) layer for two configurations, parallel and antiparallel magnetisations of adjacent ferromagnetic layers $\left.{ }^{5}\right] \ldots 9$

2.7 Plot of resistivity against temperature for $\mathrm{La}_{0.75} \mathrm{Ca}_{0.25} \mathrm{MnO}_{3}$, at various applied magnetic fields. We see that the resistivity is suppressed with increasing magnetic field. (Adapted from reference 6). . . . . . . . . . . 10

2.8 Magnetoresistance results for a nickel $(\mathrm{Ni})$ nanocontact as a function of magnetic field. The inset diagrams show a plausible model of domain shifting with the application of a magnetic field in an attempt to describe the experimental data. (Reproduced from reference 7). . . . . . . . . . . . 11

2.9 A rectangular semiconducting slab of length $\mathrm{L}$ and width $\mathrm{W}$ with a current (I) forced to flow between the left and right contacts. A magnetic field (B) is applied along the $\mathrm{z}$ axis, with the resultant Lorentz force creating a Hall angle $\left(\theta_{\mathrm{H}}\right)$ to arise between the electric field (E) and the current (I). Geometrical magnetoresistance arises for such a system with $\mathrm{L} \leq \mathrm{W}$. (Adapted from reference 8$). \ldots \ldots \ldots \ldots \ldots \ldots$ 
2.10 A circular geometry EMR device containing an outer disk of semiconducting material (InSb of radius $r_{b}$ ) and an inner embedded metallic region $\left(\mathrm{Au}\right.$ of radius $\left.r_{a}\right)$. Four contacts are situated equidistant around the disk perimeter, two contacts input and output the current while the other two measure the potential difference. The magnetic field is applied perpendicular to the plane of the $\left.\operatorname{disk}^{9}\right] \ldots \ldots \ldots \ldots$. . . . . . . . 14

2.11 Visualisation of the underlying principle responsible for the EMR effect. A low resistance regime is produced without magnetic field, with the application of a magnetic field producing a high resistance state. The current redistribution between these two states arises due to the Hall angle approaching $90^{\circ}$ for large magnetic fields. . . . . . . . . . . . . . 15

2.12 The Hall effect: here $w$ and $d$ are the width and depth of the sample respectively $(A=w \times d) ; l$ is the length of the sample; $V_{\mathrm{H}}$ is the Hall voltage; $E_{x}$ is the applied electric field producing $J_{x}$ the current density $(J=I / A) ; E_{y}$ is the transverse Hall electric field; $v$ is the velocity of the charge carriers; and $F_{L}$ is the Lorentz force. In this illustration the charge carriers are assumed to be negatively charged (electrons). . . . . . . . . 16

3.1 Six circular geometry experimental EMR devices produced by Solin et al. each with a different value of filling factor $(\alpha)$. Here, the outer semiconducting disk $\left(r_{b}=0.5 \mathrm{~mm}\right)$ contains an embedded metallic inhomogeneity including four electrical contacts $\left.{ }^{9}\right] \ldots \ldots \ldots \ldots \ldots \ldots$

3.2 Experimental magnetoresistance data produced by Solin et al., where the magnetoresistance is plotted as a function of applied magnetic field for various values of filling factor $(\alpha=0 / 16 \square, 8 / 16 \nabla, 9 / 16 \nabla, 12 / 16 \bigcirc$, $13 / 16 \bullet, 14 / 16 \diamond, 15 / 16 \diamond)$. (Reproduced from reference 9). . . . . . . 28

3.3 Experimental magnetoresistance data produced by Solin et al., where the magnetoresistance is plotted as a function of filling factor $(\alpha)$ in various applied magnetic fields $(\mathrm{H}=0.05 \mathrm{~T} \bullet, 0.1 \mathrm{~T} \triangle, 0.25 \mathrm{~T} \boldsymbol{\nabla}, 1.0 \mathrm{~T} \boldsymbol{\Delta}$ and $5 \mathrm{~T}$

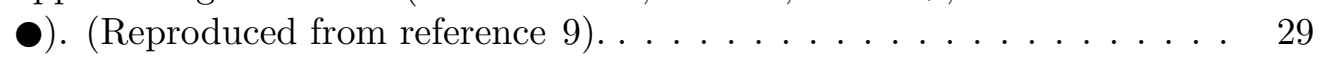

3.4 Experimental magnetoresistance results from Suh et al., where the magnetoresistance is plotted as a function of applied magnetic field for five values of filling factor $(\alpha=0.2,0.3,0.45,0.6$ and 0.7$)$. (Reproduced from reference 10) . . . . . . . . . . . . . . . . . . 30

3.5 EMR system geometry used by Suh et al., where the disk diameter is $0.1 \mathrm{~mm}$ and the filling factor is $\alpha=0.7$. The system is comprised of an outer InSb disk with an embedded Au droplet, with four contacts placed around the perimeter of the disk. (Reproduced from reference [10). . . . . 32

3.6 Magnetoresistance as a function of magnetic field for a circular geometry graphene based EMR device containing a Ti/Au metallic inclusion for various values of gate voltage (shown in the key in units of volts). The magnetoresistance measurements were carried out at $4.2 \mathrm{~K}$ and we see a maximum value of $600 \%$ in a magnetic field of $12 \mathrm{~T}$. (Reproduced from reference 11). . . . . . . . . . . . . . . . . . . . . 33

3.7 Scanning electron microscope image of the experimental EMR systems of Lu et al. indicating the four contacts placed around the perimeter of a graphene disk with a central embedded palladium conducting inclusion (indicated by red lines). Here, the metallic inclusion is not centred exactly in the graphene disk. (Reproduced from reference 12). $\ldots . . . . . . .34$ 
3.8 Magnetoresistance as a function of magnetic field for EMR systems of Lu et al. with the system geometry as given in Figure 3.7 | with filling factor of 3/4. Results are given for three values of gate voltage: $-8 \mathrm{~V}$ (black); $-6 \mathrm{~V}$ (red); and -3V (green). Here, the experimental data is represented by the solid lines with the empty circles representing simulations. The asymmetric results are due to the metallic region being misaligned from the centre of the graphene disk. (Reproduced from reference 12). . . . . . 35

3.9 System resistance as a function of magnetic field for various values of filling factor: (left) the experimental results from Solin et al. where the filling factors were: $16 \alpha=0(\square) ; 6(\mathbf{\square}) ; 8(\nabla) ; 9(\mathbf{\nabla}) ; 10(\triangle) ; 11(\mathbf{\Delta}) ; 12$ $(\bigcirc) ; 13(\bullet) ; 14(\diamond)$; and $15(\diamond)$. (Right) the results obtained from the FEM model (here the numbers represent values of $16 \alpha$ ). (Adapted from

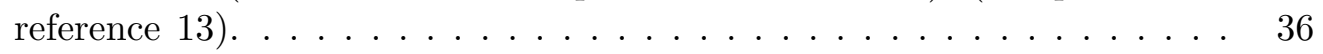

3.10 The current flow in a modified van der Pauw disk geometry, the outer disk consisting of InSb with a concentric Au metallic inclusion: (a) zero magnetic field and (b) magnetic field of 1T. The lengths of the arrows are not to scale. (Adapted from reference 13). . . . . . . . . . . . . 36

3.11 Simulated EMR disk geometry based on the systems of Solin et al. showing the four point contacts placed equidistant around the perimeter of a semiconducting disk (of radius $r_{b}$ ). The metallic droplet (of radius $r_{a}$ ) is embedded into the centre of the semiconductor. The current is input into the system through contact $\mathrm{A}$ and output at contact $\mathrm{B}$. The resulting potential difference across contacts $\mathrm{C}$ and $\mathrm{D}$ is then measured. This system shows a filling factor of $12 / 16 . \ldots \ldots \ldots \ldots$. . . . . . . . . . . . . . .

3.12 A typical mesh used for simulations for a system with a filling factor of $12 / 16$. Here, the mesh is more refined around the system perimeter and at the semiconductor-metal interface. The mesh consists of approximately 60,000 triangular mesh elements and has been refined around the current contacts (A and B) where the variation in potential is greatest. . . . . . . 38

3.13 Magnetoresistance as a function of magnetic field for simulations based on circular EMR systems, for filling factors from $1 / 16$ to $7 / 16$. . . . . . . 40

3.14 Magnetoresistance as a function of magnetic field for simulations based on circular EMR systems, for filling factors from 8/16 to 15/16. . . . . . . 41

3.15 Magnetoresistance as a function of filling factor for a circular EMR device for five values of magnetic field: 0.05T $(\bullet)$; 0.1T $(\triangle)$; 0.25T $(\boldsymbol{\nabla})$; $1 \mathrm{~T}(\mathbf{\Delta})$; and $5 \mathrm{~T}(\bullet)$. The experimental results of Solin et al. for these systems are presented in Figure 3.3 for comparision. . . . . . . . . . . . . . . . . . 42

3.16 Visualisation of the current flow and the distribution of electrical potential throughout a modified van der Pauw disk with circular conducting region $(\alpha=12 / 16)$, produced via modelling in Comsol Multiphysics. Here, the background colour represents the distribution of electrical potential with the coloured bar showing the corresponding voltages (in units of volts). The current density is represented by both the green streamlines and the black arrows. The streamlines connect regions with the same current density, while the arrows show the magnitude and direction of the current flow at various points throughout the system. The six images in this figure represent different applied magnetic fields: (a) 0T; (b) 0.5T; (c) 1T; (d)

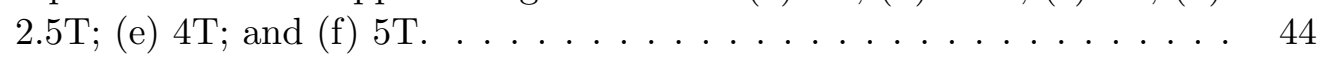


3.17 Current cross-section of a circular EMR system with a magnetic field of 0T and 5T. Here, the diameter shown in green on the diagram indicates the cross-section that was taken. This line corresponds to the $\mathrm{x}$ axis of the plot with specific points of interest highlighted by the numbers 1 to 4. The current density (normalised by the largest value in each case) is plotted along this cross-section for magnetic fields of 0T and 5T. . . . . . 48

3.18 Visualisation of the current flow and the electric field lines throughout a modified van der Pauw disk with circular conducting region $(\alpha=12 / 16)$, produced via modelling in Comsol Multiphysics. Here, the electric field lines are shown in blue, while the red arrows represent the magnitude and direction of the current flow at various points throughout the system. The three images in this figure represent different applied magnetic fields: (a) 0T; (b) 0.5T; and (c) 5T. This figure shows how the Hall angle arises in such a system. At zero magnetic field the current and the electric field are aligned parallel (zero Hall angle). At an intermediate field of 0.5T we see a non-zero Hall angle, here measured as $66^{\circ}$. Finally, with a large magnetic field of $5 \mathrm{~T}$ we see the current and the electric field are aligned almost perpendicular $\left(\right.$ Hall angle $\left.\approx 90^{\circ}\right) . \ldots \ldots \ldots$. . . . . . . . 49

3.19 Magnetoresistance as a function of magnetic field for the FEM model and the corresponding experimental data for the systems of Suh et al. respectively. The system responsible for these results was an order of magnitude smaller than the systems of Solin et al. $\left(r_{b}=0.05 \mathrm{~mm}\right)$ with a filling factor of 0.6. The device was created using semiconducting InSb and metallic $\mathrm{Au}$ with the magnetoresistance measured from 0T to 5T. The material parameters used for simulation were taken from the experimental data and can be seen in Table $3.2 . \ldots \ldots \ldots \ldots$. . . . . . . . . . . 51

3.20 Magnetoresistance as a function of magnetic field for the FEM model and the corresponding experimental data for the systems of $\mathrm{Lu}$ et al. respectively. The system responsible for these results has a filling factor of $3 / 4$. The device was created using semiconducting graphene and metallic $\mathrm{Pd}$ with the magnetoresistance measured from 0T to 9T. The material parameters used for simulation were taken from the experimental data and can be seen in Table|3.3]. . . . . . . . . . . . . . . . . 52

4.1 Diagram outlining the transformation between a circular and linear EMR device. A more detailed analysis of this transformation can be found in Appendix $|\mathrm{B}|$ (Adapted from reference 14). . . . . . . . . . . . . . . 55

4.2 A schematic diagram of a linear geometry EMR hybrid configured for a four probe measurement. I and V refer to current and voltage probes respectively. (Reproduced from reference 15). . . . . . . . . . . . . . . 56

4.3 An electron micrograph of a linear geometry EMR hybrid. The semiconducting (InSb) region is capped by $\mathrm{Si}_{3} \mathrm{~N}_{4}$ with a gold metallic shunt and four contacts. (Reproduced from reference 15). . . . . . . . . . . . . . . 56

4.4 The magnetoresistance of a linear EMR device with symmetric contact configuration for various values of magnetic field: (a) as a function of electron mobility with $\mathrm{W} / \mathrm{L}$ fixed at 0.1 (b) as a function of relative semiconductor width $(\mathrm{W} / \mathrm{L})$ for a fixed mobility of $2.09 \mathrm{~m}^{2} /$ Vs. Experimental results are depicted by the open diamonds $(\diamond)$ with the lines representing data from a FEM model. (Reproduced from reference 16). . . . . . . . . 57 
4.5 Here, the current flow in a linear EMR device ( $m$ and sc correspond to the metallic and semiconducting regions respectively) is indicated at four values of magnetic field. The white lines connect points with the same current density between the two indicated current contacts. The mobility of the semiconducting material in this device was $40,200 \mathrm{~cm}^{2} /$ Vs. (Adapted

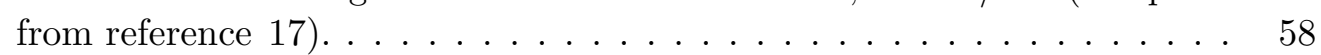

4.6 A sketch of a linear semiconductor-metal hybrid. Here, the current contacts are labelled I+ and I- while four positions of voltage probes are shown. Measuring the voltage with probes V2 and V4 constitutes a symmetric IVVI configuration. Measuring the voltage with probes V2 and V3 is an example of an asymmetric IVVI configuration. Measuring the voltage with probes V1 and V2 constitutes a VIVI configuration. (Reproduced from reference 18) . . . . . . . . . . . . . . . . . . . . . 60 60

4.7 Resistance as a function of magnetic field (theoretical and experimental data) for a linear semiconductor-metal hybrid device with two contact configurations. The labels have been altered to be consistent with Figure 4.6. The configuration labelled V2-V4 is a symmetric IVVI contact configuration, while the configuration labelled V3-V4 is an asymmetric IVVI contact configuration. $\mathrm{B}_{\text {bias }}$ is the field at which the minimum resistance occurs. Here, $\mathrm{B}_{\text {bias }}=40 \mathrm{mT}$. (Adapted from reference [17).] . . 61

4.8 EMR system geometries where the shape of the metallic region has been varied while keeping the filling factor fixed $(\alpha=8 / 16)$. These geometries dramatically influence the magnetoresistance values produced in such

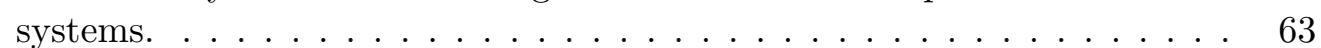

4.9 The magnetoresistance as a function of applied magnetic field for 9 EMR devices (as seen in Figure 4.8) each with a different shaped metallic region for a filling factor of $\alpha=8 / 16$. It is apparent that the shape of the conducting region has a dramatic impact on the magnetoresistance produced in each case. . . . . . . . . . . . . . . . . . . . 64

4.10 Normalised magnetoresistance as a function of applied magnetic field for 9 EMR devices (as seen in Figure 4.8) each with a different shaped metallic region for a filling factor of $\alpha=8 / 16$. Here, the functional form of the magnetoresistance is found to be dependent on the system geometry. . . 65

4.11 The factor of geometrical enhancement of the magnetoresistance between an EMR device with a branched metallic region over the circular metallic region (see Figure 4.8 (a) and (i)) as a function of magnetic field. The geometrical enhancement is huge at large magnetic fields reaching over four orders of magnitude. . . . . . . . . . . . . . . 66

4.12 Visualisation of the current flow and the distribution of electrical potential throughout a modified van der Pauw disk with a multi-branched conducting region $(\alpha=8 / 16)$, produced via modelling in Comsol Multiphysics. Here, the background colour represents the distribution of electrical potential with the coloured bar showing the corresponding voltages (in units of volts). The current density is represented by both the green streamlines and the black arrows. The streamlines connect regions with the same current density, while the arrows show the magnitude and direction of the current flow at various points throughout the system. The six images in this figure represent different applied magnetic fields: (a) 0T; (b) 0.5T; (c)

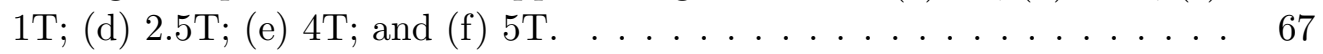


4.13 A cross-section showing the variation in current density (normalised) along the vertical line throughout the system for a multi-branched EMR device (as seen in Figure 4.8(i)). The points 1 to 10 on the cross-section correspond to points 1 to 10 on the $\mathrm{x}$ axis of the plot. . . . . . . . . 71

4.14 System geometry of a linear EMR device. Conformal mapping has shown that equivalent linear devices can be produced from circular geometry devices. This device has similar features to those of the circular devices as seen in Figure 4.8 (a). Here, we see a semiconducting region with four contacts placed along the outside of the device. In this geometry the metallic region is said to be external as opposed to internal in the circular

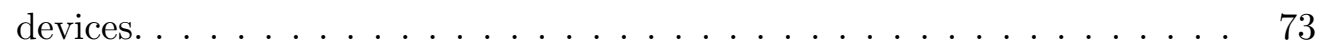

4.15 Modelled magnetoresistance as a function of applied magnetic field for a linear EMR device. The system responsible for these results has a filling factor of 0.866 (see Figure 4.14) and material parameters given in Table 3.1 . 74

4.16 Visualisation of the current flow and the distribution of electrical potential throughout a linear EMR device $(\alpha=0.866)$, produced via modelling in Comsol Multiphysics. Here, the background colour represents the distribution of electrical potential with the coloured bar showing the corresponding voltages (in units of volts). The current density is represented by both the green streamlines and the black arrows. The streamlines connect regions with the same current density, while the arrows show the magnitude and direction of the current flow at various points throughout the system. The six images in this figure represent different applied magnetic fields: (a) 0T; (b) 0.5T; (c) 1T; (d) 2.5T; (e) 4T; and (f) 5T. . . . . . . . . . . 75

4.17 A cross-section showing the variation in current density (normalised) along the vertical line throughout the system for a linear geometry EMR device with filling factor $\alpha=0.866$ (as seen in Figure 4.14). The points 1 to 3 on the cross-section correspond to points 1 to 3 on the $\mathrm{x}$ axis of the plot. . . 79

5.1 The influence of the metal resistivity on the resistance of a linear EMR device (FEM model) for two values of magnetic field, 25mT (red solid line) and 50mT (green dashed line). (Reproduced from reference 19). . . . 82

5.2 Magnetoresistance as a function of the conductivity ratio $\left(\sigma_{M} / \sigma_{S}\right)$ for a linear EMR device (FEM model with $\alpha=0.91)$ for two values of magnetic field, 0.05T (filled squares) and 5T (open circles). (Reproduced from

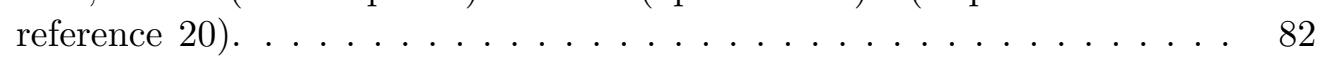

5.3 The resistance as a function of applied magnetic field for three values of semiconductor mobility: $12.4 \mathrm{~m}^{2} / \mathrm{Vs}$ (red solid line); $24.8 \mathrm{~m}^{2} / \mathrm{Vs}$ (green dashed line); and $49.6 \mathrm{~m}^{2} / \mathrm{Vs}$ (blue dashed line). These results were created from a FEM model with a constant carrier density for a linear geometry EMR device. (Reproduced from reference 19). . . . . . . . . . . . . . 84

5.4 Magnetoresistance as a function of semiconductor mobility at four values of magnetic field $(100 \mathrm{mT}, 250 \mathrm{mT}, 500 \mathrm{mT}$ and $1 \mathrm{~T})$ showing the comparison to experimental data. These results were created from a FEM model with a constant carrier density for an EMR device of a linear geometry. (Reproduced from reference 16). . . . . . . . . . . . . . . . . 84 
5.5 Magnetoresistance as a function of semiconductor mobility for two values of magnetic field, 0.05T (filled squares) and 5T (open circles) for a FEM model of a linear EMR system. We see a quadratic dependence of the magnetoresistance with mobility at low field and signs of saturation for large mobilities at high fields. (Reproduced from reference 20). . . . . . . 85

5.6 The introduction of an intermediate layer in order to model the contact resistance at the semiconductor-metal interface in a linear EMR system. (Reproduced from reference 19). . . . . . . . . . . . . . . . . . 86

5.7 Resistance of a linear EMR device as a function of magnetic field for various values of contact resistance, compared to experimental data. The largest value of contact resistance dramatically increases the system resistance at all magnetic fields. (Reproduced from reference 19). . . . . . . . . . . . 87

5.8 Magnetoresistance as a function of contact resistivity (shown in blue) for a FEM model of a linear geometry EMR device, at an applied magnetic field of 1T. We see that large values of contact resistivity dramatically reduce the EMR effect. (Reproduced from reference 21 ). . . . . . . . . . 87

5.9 Magnetoresistance as a function of applied magnetic field for various values of conductivity ratio $\left(\sigma_{M} / \sigma_{S}\right.$, see legend) for a modified van der Pauw disk with filling factor of $8 / 16$. We see that the greatest magnetoresistance values arise for large values of conductivity ratio (when the conductivity of the metal is significantly greater than that of the semiconductor). The magnetoresistance for the two largest values of conductivity ratio are almost indistinguishable from one another on this plot. . . . . . . . . . 88

5.10 Magnetoresistance against conductivity ratio $\left(\sigma_{M} / \sigma_{S}\right)$ for three values of magnetic field: 1T (red); 2T (green); and 5T (blue). These results are for the same system as in Figure $|5.9|$ with filling factor of $8 / 16$. In order for a significant magnetoresistance to arise the conductivity ratio is required to be larger than two orders of magnitude. The systems studied by Solin et al. have a conductivity ratio of 2,430 and are therefore well within this

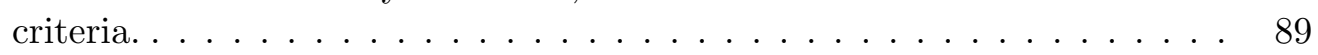

5.11 Magnetoresistance as a function of applied magnetic field for various values of semiconductor mobility for a modified van der Pauw disk with filling factor of 8/16. Here, the mobility of the metallic region was fixed at $\mu_{M}$ $=50 \mathrm{~cm}^{2} / \mathrm{Vs}$ (the same as that used in Solin et al. experiments). We see that larger values of semiconductor mobility result in the magnetoresistance saturating at a smaller magnetic field and thus producing larger magnetoresistance values at a given field (below the saturation point). The semiconductor mobility in the systems of Solin et al. was: $\mu_{S}=$

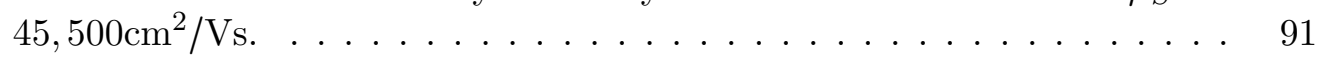

5.12 Magnetoresistance against semiconductor mobility for two values of magnetic field, 0.05T (red filled squares) and 5T (blue open circles). The system responsible for these results is the same as in Figure 5.11 with a filling factor of $8 / 16$. The lines have been fitted in order to visualise the trend in each case. At 0.05T we see a quadratic dependence of the magnetoresistance with semiconductor mobility. . . . . . . . . . . . 92 
5.13 Magnetoresistance against dimensionless magnetic field $(\mu B)$ for a modified van der Pauw disk with filling factor of 8/16: (a) and (b) present the low and high magnetic field cases. Here, the results for the nine values of semiconductor mobility presented in Figure [5.11 are found to overlap when plotted against the dimensionless magnetic field, resulting in a single curve. In (a), at low values of $\mu B$, we see the magnetoresistance increase quadratically, while saturation of the magnetoresistance can be observed in (b) for high magnetic fields. . . . . . . . . . . . . . . . . . . 93

5.14 The geometry of a modified van der Pauw disk used to model the effect of a contact resistance at the semiconductor-metal interface. The system consists of an outer semiconducting disk (1mm diameter) with a concentric embedded metallic inclusion (filling factor of 13/16). A thin intermediate layer (thickness of $5 \mu \mathrm{m}$ ) is introduced at the semiconductor-metal interface, with four contacts placed equidistant around the disks perimeter. . . . . . 9 94

5.15 Magnetoresistance as a function of applied magnetic field for various values of semiconductor-metal contact resistivity. The system responsible for these results (see Figure 5.14) has a filling factor of 13/16 and contains an intermediate contact layer with thickness of $5 \mu \mathrm{m}$. The experimental data (extracted) is shown in red and the corresponding system without the intermediate interface is shown in blue. We see that, consistent with the mechanism for the effect, larger values of contact resistivity reduce the magnetoresistance. We find that an interface resistivity of $1.55 \times 10^{-4} \Omega \mathrm{m}$ was required to fit the model to the experimental data, thus giving an overestimate of the system's interface resistivity. . . . . . . . . . . . 95

5.16 Here, the current flow is presented for four circular modified van der Pauw disks (all with filling factor of 13/16): (a) and (b) represent a system with contact resistivity of $1.55 \times 10^{-4} \Omega \mathrm{m}$ at magnetic fields of 0 and $5 \mathrm{~T}$ respectively. While (c) and (d) represent a system with contact resistivity of $0.1 \Omega \mathrm{m}$ at magnetic fields of 0 and 5T respectively. We see that the EMR mechanism still holds for small values of interface resistance while large values cause the current to flow through the semiconducting disk in both the high (5T) and low (0T) field regimes. This explains the drop in magnetoresistance with increased interface resistance as observed in

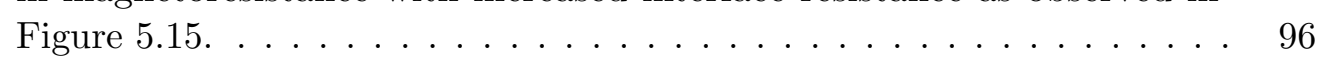

6.1 Magnetoresistance for $\mathrm{Ag}_{2+\delta} \mathrm{Se}(\delta=0.01)$ as a function of applied magnetic field $(1 \mathrm{~T}=10 \mathrm{kOe})$ for various values of temperature in the range of 4.5 to $300 \mathrm{~K}$. The insert shows the linear field dependence continuing down to fields of a few Oe. (Reproduced from reference 22). . . . . . . . . . . . . 100

6.2 Magnetoresistance for $\mathrm{Ag}_{2+\delta} \mathrm{Se}\left(\delta=10^{-4}\right)$ as a function of applied magnetic field (up to $55 \mathrm{~T}$ ) for various values of temperature in the range of $1.5 \mathrm{~K}$ to 290K. (Reproduced from reference 23). . . . . . . . . . . . . . . . 101

6.3 The microstructure of a silver chalcogenide compound showing the coexistence of silver nanoparticles and silver paths. (Reproduced from reference

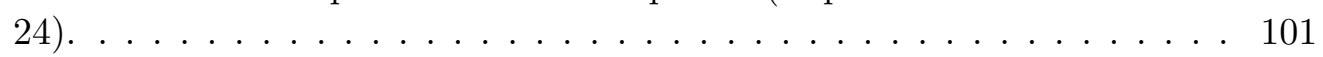

6.4 Two-dimensional four terminal resistor network of Parish and Littlewood. Showing (a) a four terminal resistor consisting of a homogeneous disk with four equally spaced contacts on the perimeter and (b) the resultant four terminal resistor network. (Adapted from reference 25). . . . . . . . . 103 
6.5 The system geometry of the inhomogeneous random branch and droplet model (RBDM) with filling factor $\alpha=0.32$ (10\% of the system area is metallic). An equal area of silver metal is used to produce randomly sized, positioned and orientated branches with the other half forming randomly sized and positioned droplets in a semiconducting $\mathrm{Ag}_{2}$ Se disk (diameter

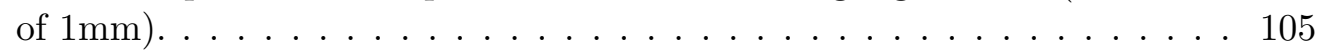

6.6 The system geometry of the inhomogeneous $10 \times 10$ grid model $(0.5 \mathrm{~mm}$ square) with filling factor $\alpha=0.32$. Here, 10 of the 100 squares are randomly assigned as silver metal with the remaining squares semiconducting $\mathrm{Ag}_{2} \mathrm{Se} .106$

6.7 Magnetoresistance as a function of magnetic field for: the RBDM (with filling factor of $\alpha=0.32$ as seen in Figure 6.5); the 10x10 grid model (with filling factor of $\alpha=0.32$ as seen in Figure 6.6 ; ; and experimental data for

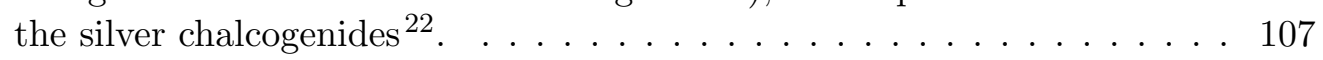

6.8 Visualisation of the current flow throughout a RBDM system (with filling factor of $\alpha=0.32$ as seen in Figure 6.5 ) produced via modelling in Comsol Multiphysics. The background colour represents the two phases, silver metal (pink) and semiconducting $\mathrm{Ag}_{2} \mathrm{Se}$ (cyan). The current density is represented by both the blue streamlines and black arrows. The streamlines connect regions with the same current density, while the arrows show the magnitude and direction of the current flow at various points throughout the system. The four images in this figure represent different applied magnetic fields: (a) 0T; (b) 3T; (c) 5.5T; and (d) 50T. . . . . . . . . . . 109

6.9 Visualisation of the current flow throughout a 10x10 grid model system (with filling factor of $\alpha=0.32$ as seen in Figure 6.6 produced via modelling in Comsol Multiphysics. The background colour represents the two phases, silver metal (pink) and semiconducting $\mathrm{Ag}_{2} \mathrm{Se}$ (cyan). The current density is represented by both the blue streamlines and black arrows. The streamlines connect regions with the same current density, while the arrows show the magnitude and direction of the current flow at various points throughout the system. The four images in this figure represent different applied magnetic fields: (a) 0T; (b) 3T; (c) 5.5T; and (d) 50T. . . . . . . 113

6.10 The total current density (normalised) along a small cross-section of a RBDM system (as seen in Figure 6.5) for two values of magnetic field, 0T and 50T. Here, points 1 to 4 on the vertical cross-section correspond to points 1 to 4 on the $\mathrm{x}$ axis of the plot. . . . . . . . . . . 118

6.11 The total current density (normalised) along a small cross-section of a 10x10 grid model system (as seen in Figure 6.5) for two values of magnetic field, 0T and 50T. Here, points 1 to 4 on the vertical crosssection correspond to points 1 to 4 on the x axis of the plot. . . . . . . . 119

6.12 Multiple configurations of the RBDM (see (a) - (d)) and the 10x10 grid model (see (e) - (h)) presented alongside the corresponding magnetoresistance for each case as a function of magnetic field (see (i) and (j) for the RBDM and 10x10 grid model respectively). Configurations (a) and (e) are the ones that have been investigated in more detail in this chapter, as seen in Figures 6.5 and $\mid 6.6$. All configurations have a filling factor of $\alpha=0.32$ and the same material parameters as given in Table 6.1$] \ldots . .121$ 
A.1 (a) An arbitrary shaped disk: the van der Pauw method requires two resistance measurements in order to determine the sheet resistivity. (b) A disk containing a line of symmetry: this simplification allows the sheet resistivity to be obtained with only one resistance measurement 26 27 $]$. . . 127

A.2 The van der Pauw method for a circular disk can be used to determine the resistivity of the material with the need for only one resistance measurement. Here, the current streamlines (continuous lines) and equipotentials (dashed lines) in the system are presented. (Adapted from reference 28). 127

A.3 The three cases of non-ideal electrical contacts where the resultant errors were considered by van der Pauw 27$]$. . . . . . . . . . . . . . . 128

B.1 Mapping of a unit circle (R1) centred at the origin and various points of interest from the complex t-plane to the complex z-plane with the use of the bilinear transformation. Points inside the circle correspond to points in the positive half of the complex z-plane. . . . . . . . . . . . . 130

B.2 The addition of a line with $\operatorname{Im}(\mathrm{z})=\mathrm{y} 2$ in the z-plane eliminates all values of $\mathrm{z}$ with $\operatorname{Im}(\mathrm{z})>\mathrm{y} 2$ thus making the device finite in the $\operatorname{Im}(\mathrm{z})$ direction. This line corresponds to a circle in the t-plane (R2). . . . . . . . . . 132

B.3 The addition of two vertical lines in the $\mathrm{z}$-plane $(\operatorname{Re}(\mathrm{z})=\mathrm{x} 3$ and $\operatorname{Re}(\mathrm{z})=\mathrm{x} 4)$ eliminate all values of $\mathrm{z}$ with $\operatorname{Re}(\mathrm{z})>\mathrm{x} 3$ and $\operatorname{Re}(\mathrm{z})<\mathrm{x} 4$, producing a finite sized rectangular device in the z-plane. These two lines correspond to two

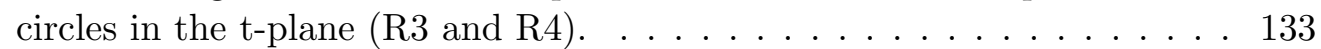

B.4 Introducing a second horizontal line in the $\mathrm{z}$-plane at $\operatorname{Im}(\mathrm{z})=\mathrm{y} 5$ produces a rectangular device with two separate regions. This line corresponds to the circle R5 in the t-plane and thus showing how a circular geometry can be mapped into a linear rectangular one. . . . . . . . . . . . . . . . 134 


\section{List of Tables}

3.1 Material parameters of the EMR systems of Solin et al. at zero magnetic

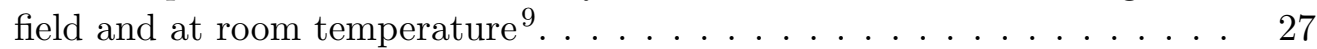

3.2 Material parameters of the EMR systems of Suh et al. at zero magnetic field and at room temperature $10 \ldots \ldots \ldots \ldots \ldots \ldots \ldots$

3.3 Material parameters of the EMR systems of Lu et al. at zero magnetic field and at room temperature $12 \ldots \ldots \ldots \ldots$. . . . . . . . . 34

6.1 Material parameters of the silver chalcogenide materials at zero magnetic field and at room temperature $22 \ldots \ldots \ldots \ldots$. . . . . . . . . . . . .

A.1 Approximate values of the function $f$, which is required to measure the resistivity of a sample of arbitrary shape via the van der Pauw method ${ }^{26 \mid 27 / 29}$. 126

A.2 Summary of the error in the resistivity originating from three specific cases of non-ideal contacts within the van der Pauw method 27$] \ldots$. . . . 128

B.1 The real and imaginary components of corresponding points in the $\mathrm{t}$ and z-planes as seen in Figure|B.1 . . . . . . . . . . . . . . . . 131 


\section{Chapter 1}

\section{Introduction}

Magnetoresistance is a measure of the change in electrical resistance of a material when a magnetic field is applied ${ }^{30}$. This phenomonen was first discovered by William Thompson (more commonly known as Lord Kelvin) in 1857 when he was investigating how the electrical resistance of iron and nickel were influenced by the application of a magnetic field:

"I found that iron, when subjected to magnetic force, acquires an increase of resistance to the conduction of electricity along, and a diminution of resistance to the conduction of electricity across, the lines of magnetisation ... I have ascertained that the electric conductivity of nickel is similarly influenced by magnetism." "31

In general the effect is small in pure metals with the magnetoresistance of copper reaching only approximately $0.25 \%$ in a large magnetic field of $10 \mathrm{~T}$ at room temperature ${ }^{1}$. At low temperatures the effect is enhanced; at $4.2 \mathrm{~K}$ copper produces a magnetoresistance of $1,307 \%$ in a magnetic field of $10.1 \mathrm{~T}^{1}$. The effect is generally not as small in semiconductors and in the semi-metal Bismuth $\underline{32}$.

Many larger magnetoresistance effects (often orders of magnitude larger than the effect discovered by Thompson) have been discovered in more complex systems. Typically, a certain aspect of a specifically designed hybrid system is exploited in order to produce a large magnetoresistance effect. Such magnetoresistance effects include: Giant (GMR); Colossal (CMR); Tunnelling (TMR); Ballistic (BMR)*; and Geometrical magnetoresistance. These effects have stimulated a great deal of interest in magnetoresistive systems

${ }^{*}$ It is now believed that the BMR effect is in fact an artefact due to motion on the atomic scale and not a reproducible magnetoresistance effect, see Chapter 2 
since the original discovery by Thompson (including speculation surrounding the name of the next effect!). The quest to discover new magnetoresistance effects is not only driven by an academic desire to understand the underlying mechanism but also due to the huge possibilities for practical applications.

Traditionally, magnetoresistive materials have been used as magnetic field sensors which have a vast number of applications spanning many fields, such as: digital information storage; medical devices; automotive control systems; and consumer electronics 14 . One of the most well known of these applications is the use of the GMR effect in the read heads of the computer hard disk drives that are found almost ubiquitously in the modern world. For the discovery of the GMR effect Albert Fert and Peter Grünberg shared the 2007 Nobel Prize in Physics. Practical magnetic field sensors require materials that produce a notable magnetoresistance in a modest magnetic field at room temperature. Therefore the different systems and mechanisms responsible for the various magnetoresistance effects discovered to date, make certain effects more suitable for specific applications ${ }^{22}$.

In 2000, another large magnetoresistance effect was discovered by Solin et al. which was named extraordinary magnetoresistance (EMR) ${ }^{9}$. The EMR effect extended the geometrical magnetoresistance effect to semiconductor-metal hybrids, resulting in extremely large magnetoresistance values of over a million percent in a $5 \mathrm{~T}$ magnetic field at room temperature ${ }^{14}$. These values are orders of magnitude larger than those found in other previously discovered magnetoresistance effects and therefore sparked further research into the phenomenon. The EMR effect was discovered in composite van der Pauw disks consisting of two non-magnetic components; a conducting droplet embedded inside a semiconducting disk. These systems were subjected to a transverse magnetic field with the system resistance measured using a four probe (van der Pauw) method. The EMR effect was particularly exciting due to its great potential for applications, especially in the field of hard drive read heads.

In 1997, Xu et al. discovered a large magnetoresistance (comparable in magnitude to that of the CMR materials) in the non-magnetic silver-rich silver chalcogenides; $A g_{2+\delta} S e$ and $A g_{2+\delta} T e^{22}$. The addition of a small excess amount $(\delta)$ of silver atoms into the structure of the semiconductors $\mathrm{Ag}_{2} \mathrm{Se}$ and $\mathrm{Ag}_{2} \mathrm{Te}$ (that have no appreciable magnetoresistance themselves) caused a large magnetoresistance to be observed. This magnetoresistance was unusual not only for the magnitude of the effect but for its linear dependence on applied magnetic field which remained, even at very low magnetic fields ${ }^{22}$. At room temperature and in a magnetic field of 5.5T the magnetoresistance reached $200 \%$ with no sign of saturation. Subsequently, the microstructure of these materials has been investigated. The excess silver was found to form a branched conducting network along the grain boundaries with silver droplets also interspersed inside the semiconducting 
matrix 2433134 . This two-phase nature draws parallels with the EMR effect. Both systems contain two non-magnetic phases: one a semiconducting phase and the other conducting. Many models have since been proposed in an attempt to describe the magnetoresistance in similar disordered two-phase systems $25135,53$.

This Thesis aims to be a comprehensive investigation into systems that exhibit the EMR effect by the use of the finite element method (FEM). Chapter 2 serves to describe the background behind the EMR effect. This includes: a brief overview of previously discovered magnetoresistance effects; fundamentals of the electron transport in the presence of a magnetic field; and aspects of the system that are considered in the FEM modelling process. Chapter 3 focuses on the EMR effect in circular geometry EMR devices. This includes: a review of experimental results reported in such systems; the procedure of creating a model of a specific device; and the results produced by these models in comparison to experimental data. Chapter 4 investigates the effect of the system geometry on the magnetoresistance. Initially, previous geometrical modifications of systems that exhibit the EMR effect are reviewed. This is followed by the results of FEM models that have been produced in order to investigate how the EMR effect is influenced by the shape of the metallic region. The results of modelling an alternative linear geometry EMR device are then presented. Chapter 5 extends the investigation to include the effect on the magnetoresistance of varying the material parameters of EMR systems. This begins by reviewing previous works before presenting results of three specific changes to the material parameters: the ratio of metallic to semiconducting conductivity; the mobility of the semiconducting material; and the introduction of a finite resistance at the semiconductor-metal interface. The results are presented in context with previous studies. Chapter 6 progresses from EMR devices to more disordered inhomogeneous semiconductor-metal systems. Here, the unusual magnetoresistance of the silver chalcogenides has been considered. Two separate models are then presented which are based on a randomly distributed two-phase microstructure with the results compared to those of the silver chalcogenides. Finally, conclusions are drawn in Chapter 7 and appendices and bibliography presented. 


\section{Chapter 2}

\section{Background Concepts}

\subsection{Magnetoresistance}

Since the initial discovery of magnetoresistance in iron and nickel by Lord Kelvin in 1857 many different magnetoresistance effects have been reported in a variety of systems. Magnetoresistance is a dimensionless quantity which can either be positive or negative. The standard definition of magnetoresistance is given in Equation 2.1 with values normally quoted as a percentage.

$$
\text { Magnetoresistance }=\frac{R(\mathbf{B})-R(0)}{R(0)}=\frac{\Delta R}{R_{0}} .
$$

These magnetoresistance effects are utilised in a number of practical applications. Differences in system construction and the resulting magnetoresistance effect make certain magnetoresistance effects better suited to specific applications.

The ideas introduced in this Chapter provide a background knowledge into magnetoresistance effects. Initially, previously discovered magnetoresistance effects are considered in order to place the EMR effect in context. A brief description of the Hall effect is then presented followed by an overview of electron transport in a conductor, with and without a magnetic field. Finally, the finite element method and the general steps to produce a FEM model in Comsol Multiphysics are outlined.

\subsubsection{Ordinary Magnetoresistance (OMR)}

In non-magnetic metals the magnetoresistance is generally small (of the order of a few percent) at room temperature with larger values found at low temperatures or in 


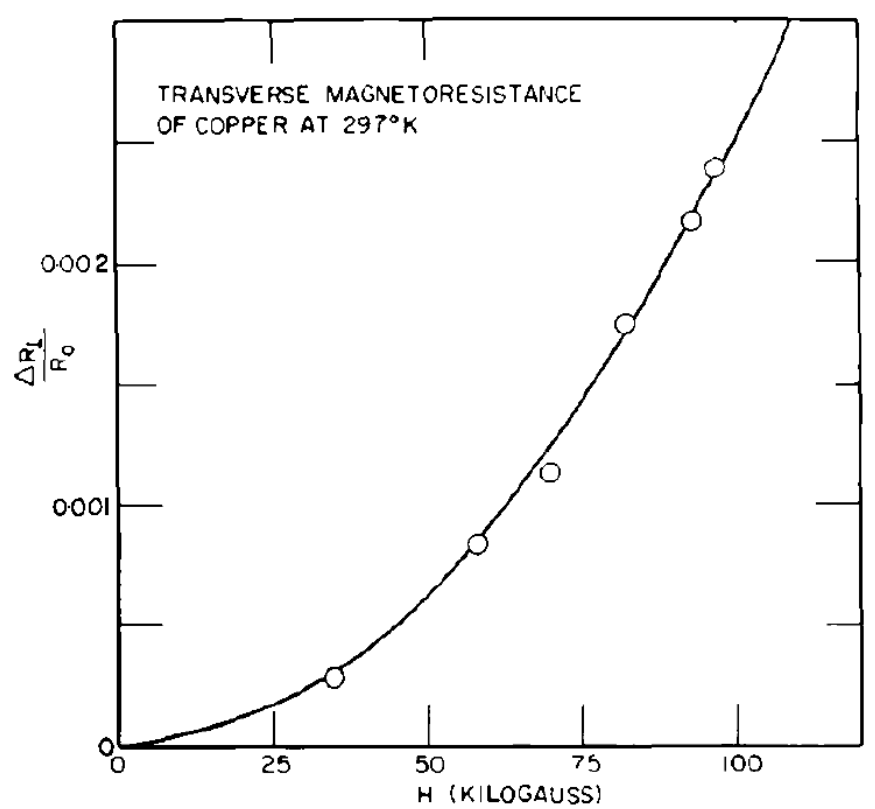

FiguRE 2.1: Magnetoresistance of copper at room temperature (297K) as a function of magnetic field (given in kilogauss, $1 \mathrm{~T}=10$ kilogauss). The experimental points are represented by the open circles while the solid line is a fitted function. The magnetoresistance reaches approximately $0.25 \%$ in a magnetic field of $10 \mathrm{~T}$. (Reproduced from reference 1).

semiconductors and the semimetal bismuth ${ }^{32}$. Figure 2.1 shows the magnetoresistance of copper at room temperature in a magnetic field of up to $10 \mathrm{~T}$. The largest value of magnetoresistance achieved is approximately $0.25 \% 1$. Without an applied magnetic field electrons in a metal travel along straight trajectories between scattering events ${ }^{32}$. The application of a magnetic field causes the Lorentz force to act on the electrons perpendicular to their direction of motion. This causes the electron trajectories to become curved or even helical between collisions. A measure of how much the electron trajectories are influenced by the magnetic field is the parameter $\omega_{c} \tau$, where $\omega_{c}$ is the cyclotron frequency and $\tau$ is the time between scattering events. Large values of $\omega_{c} \tau$ produce tight helicies allowing many turns between collisions thus affecting the conduction process considerably and increasing system resistance. The longitudinal (B \|I) and transverse $(\mathbf{B} \perp \mathrm{I})$ magnetoresistances are both positive which are proportional to $\mathrm{B}^{2}$ in weak fields $\left(\omega_{c} \tau<1\right)^{54}$. In very strong magnetic fields the magnetoresistance may do one of three things:

- The magnetoresistance saturates for all orientations of the crystal relative to the magnetic field. These materials have closed Fermi surfaces e.g. In, Al, Na and Li;

- The magnetoresistance may continue to increase up to the highest fields studied. These materials have an equal number of electrons to holes e.g. Bi, Sb, W and Mo; or 
- The magnetoresistance may saturate in some crystal directions but not in others. These materials have Fermi surfaces containing open orbits in some crystal directions e.g. $\mathrm{Cu}, \mathrm{Ag}, \mathrm{Au}, \mathrm{Mg}, \mathrm{Zn}, \mathrm{Cd}, \mathrm{Tl}, \mathrm{Sn}, \mathrm{Pb}$ and $\mathrm{Pt} 2 \frac{230}{\text {. }}$

When discussing experimental magnetoresistance results, Kohler's rule has proven to be useful. This states that magnetoresistance is a function of the ratio $\mathrm{B} / \rho_{0}$, where $\mathrm{B}$ is the magnitude of the applied magnetic field and $\rho_{0}$ is the zero field resistivity. This is generally found to be in agreement with experimental results 54 .

\subsubsection{Anisotropic Magnetoresistance (AMR)}

In ferromagnetic materials the magnetoresistance is similarly small in low magnetic fields as in OMR (order of a few percent). However, unlike OMR the effect is anisotropic, with the resistance of the system dependent on the direction of current flow relative to the magnetisation of the sample. This so-called anisotropic magnetoresistance effect was discovered in 1857 by Lord Kelvin whilst investigating the influence of a magnetic field on the electrical conductivity of nickel and iron 31 .

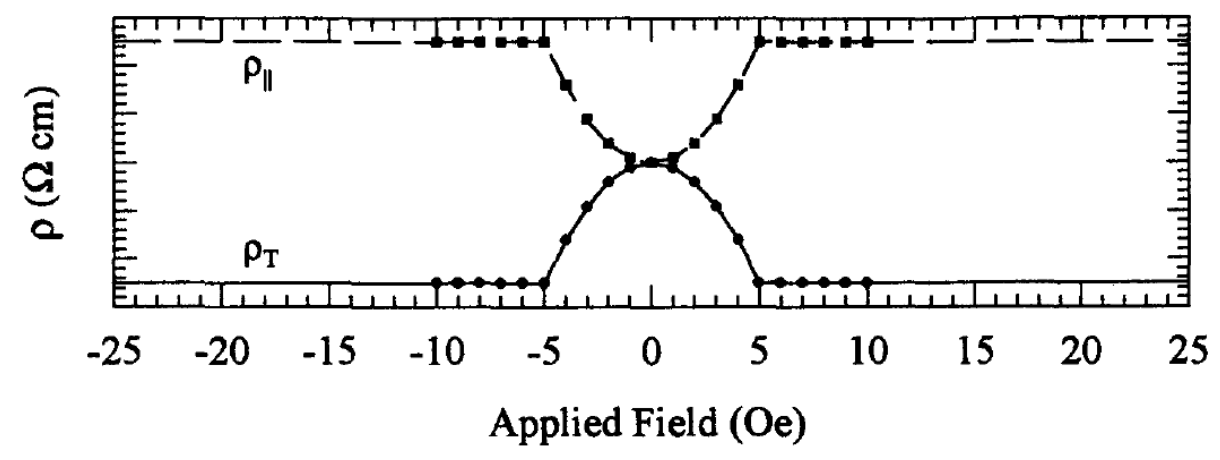

Figure 2.2: Representation of the anisotropic magnetoresistance in Permalloy, showing the difference in resistivity when the current is aligned perpendicular and parallel to the magnetic field. (Reproduced from reference 2).

The AMR effect arises as a result of spin orbit coupling. When the magnetisation of the sample is rotated (via an external magnetic field) the electron cloud around each nucleus deforms. This deformation changes the scattering cross-section of the conduction electrons thus changing the materials resistance. The sign of the AMR effect is dependent on the form of the spin orbit coupling $\sqrt[55]{ }$. However, commonly the resistance of the system is largest when the current and magnetic field are aligned parallel, as depicted in Figure 2.2. By aligning the magnetic field parallel to the direction of current flow, the bound electron orbits (around each nucleus in the crystal lattice) are in the plane perpendicular to the current flow. This results in a large scattering cross-section and a high system resistance. However, if the magnetic field and current are perpendicular, 
the orbits of the electrons are in the same plane as the current flow. This results in a low scattering cross-section, therefore a lower resistance state ${ }^{2}$. A simple visualisation of this idea is given in Figure 2.3. In fact the spin orbit origin of the AMR effect is not trivial and the sign of the effect can be opposite to that represented in Figure 2.3. The AMR effect is utilised in magnetic recording heads made from Permalloy (NiFe) which has magnetoresistance values of approximately $2 \%{ }^{2}$.
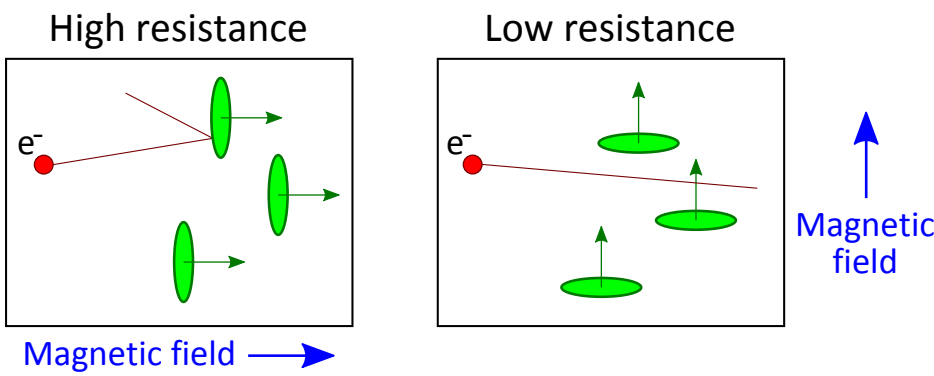

FIGURE 2.3: Illustration of the change in scattering cross-section when the current is aligned parallel with (high resistance) and perpendicular to (low resistance) the magnetic field. Here, the green ovals represent the scattering cross-section of bound electron orbits around each nucleus of the crystal lattice.

\subsubsection{Giant Magnetoresistance (GMR)}

Giant magnetoresistance (GMR) was discovered in 1988 by Fert and Grünberg (independently) for which they shared the 2007 Nobel Prize in Physics 356 . The effect was discovered in a system composed of alternating layers (typical thickness of the order of nanometers) of ferromagnetic (iron) and non-magnetic (chromium) materials. In general the effect is large and negative, typically hundreds of percent at low temperatures and an order of magnitude at room temperature ${ }^{3}$. Figure 2.4 shows the change in resistance with magnetic field for three systems, each with a different thickness of non-magnetic (Cr) layer.

The GMR effect relies upon spin dependent scattering, a process in which the scattering for one spin orientation is stronger than for the other orientation ${ }^{2}$. An electrical current contains an equal amount of spin up and spin down electrons. When electrons of both spin orientations approach a magnetised ferromagnetic layer the orientation aligned antiparallel to the magnetisation of this layer experience more effective scattering than those aligned in a parallel orientation. In a GMR material the coupling of the ferromagnetic layers can be manipulated (due to the RKKY interaction) by varying the thickness of the non-magnetic layer. With no applied magnetic field the magnetisation of adjacent ferromagnetic layers align antiparallel. This is the high resistance regime of a GMR material since both spin orientations of electrons will experience strong scattering in one 
or other adjacent ferromagnetic layers. However, the application of a large magnetic field causes parallel alignment of the magnetisations of adjacent ferromagnetic layers. This constitutes a regime of low system resistance. Here, electrons that align parallel to the magnetisation of the ferromagnetic layers will only undergo weak scattering in both layers. Therefore, only the electrons aligned antiparallel to the magnetisation of the ferromagnetic layers will undergo strong scattering. This reduces the overall resistance of the material. Figure 2.5 illustrates how this mechanism arises. The GMR effect led to a vast amount of research in the field referred to as spintronics. Since its discovery the GMR effect has been observed in many different multi-layered systems. The read heads of computer hard disk drives employ the GMR effect to read bits of information from the disk ${ }^{14}$.

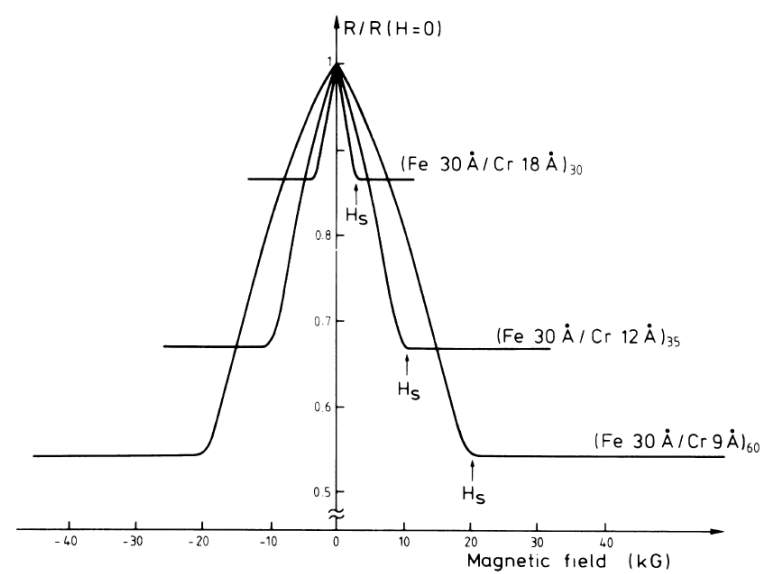

FIGURE 2.4: The initial discovery of GMR produced a maximum magnetoresistance value of approximately $50 \%$ at $4.2 \mathrm{~K}$ in a magnetic field of $2 \mathrm{~T}$. Here, a chromium $(\mathrm{Cr})$ layer of varying thickness is positioned between layers of iron $(\mathrm{Fe})$. (Reproduced from reference [3).

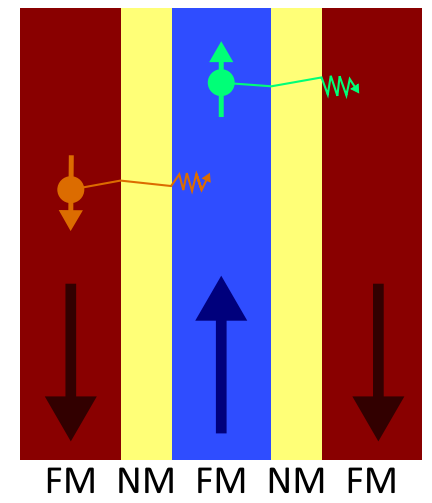

High Resistance $(B=0)$

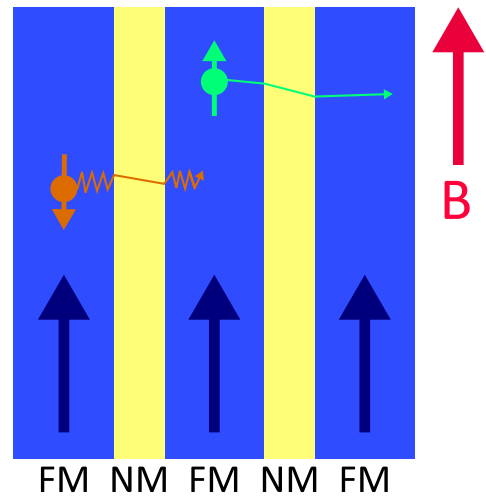

Low Resistance

$(\mathrm{B}>0)$

FiguRE 2.5: In GMR materials, applying a magnetic field changes the magnetisation of adjacent ferromagnetic (FM) layers from antiparallel to parallel. This results in different scattering processes of conduction electrons and thus a change in resistance ${ }^{4}$. 


\subsubsection{Tunnelling Magnetoresistance (TMR)}

Tunnelling magnetoresistance (TMR) is found in similar systems to that of the GMR effect. However, the non-magnetic metallic layer separating two ferromagnetic layers is replaced by a thin insulating layer (only a few atomic layers thick) creating a magnetic tunnel junction (MTJ). The effect can be very large (typically up to an order of magnitude higher than the GMR effect) with a magnetoresistance of $604 \%$ observed at $300 K^{57}$. The TMR effect was first discovered in 1975 by Julliere with a renewal of interest in the early $1990 \mathrm{~s}^{14158}$. The current flow across a MTJ is due to quantum mechanical tunnelling of electrons through the insulating layer.

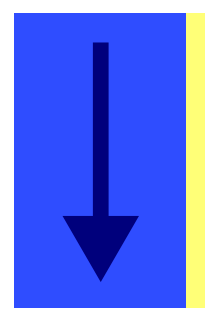

FM

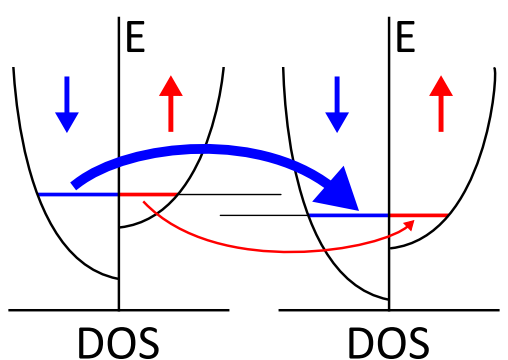

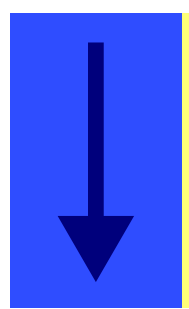

FM

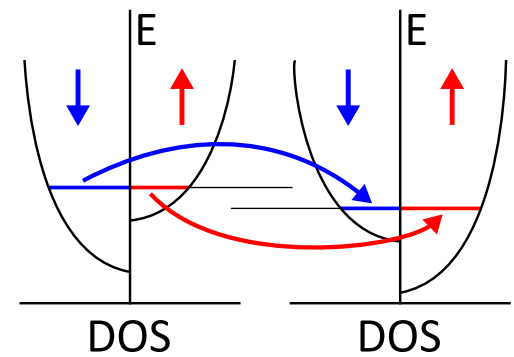

FigURE 2.6: Illustration showing tunnelling between two ferromagnetic (FM) layers separated by a thin insulating (I) layer for two configurations, parallel and antiparallel magnetisations of adjacent ferromagnetic layers ${ }^{5}$.

A magnetised ferromagnetic material has a different density of states for spin up and spin down electrons thus creating the magnetisation. Therefore, more states are available for one spin orientation than the other. When a voltage is applied across a MTJ the probability of the electron tunnelling through the insulating barrier depends on the availability of free states of the same spin orientation on the other side of the barrier. When the MTJ is in a parallel configuration the majority spin orientation (the electron spin that is aligned parallel with the magnetisation of the ferromagnetic layers) will have many available states of the same orientation on the other side of the barrier, thus a large tunnelling current flows producing a low resistance state. However, when the MTJ is in an antiparallel configuration the majority spin orientation on one side of the insulating barrier will have few states of the same orientation on the other side of 
the barrier. The minority spin orientation will have many states available on the other side of the barrier but this is not significant due to the low number of original states. Therefore, this antiparallel configuration produces a lower tunnelling current and a higher overall resistance ${ }^{4}$. An external magnetic field can be used to switch between the two configurations, therefore producing a magnetoresistance effect. Figure 2.6 illustrates how this mechanism arises. This type of magnetoresistance is a potential source of next generation read heads in future hard disk drives ${ }^{14}$.

\subsubsection{Colossal Magnetoresistance (CMR)}

Colossal magnetoresistance (CMR) occurs in manganese perovskites. These materials are very similar to those of the cuprate superconductors, except here manganese replaces copper resulting in very different electrical properties. A typical structure of a CMR material is $\mathrm{RE}_{1-x} \mathrm{AE}_{x} \mathrm{MnO}_{3}$ where $\mathrm{RE}$ is a trivalent rare earth metal (e.g. La, Pr, Nd, $\mathrm{Sm}$ ) and AE represents a divalent alkaline earth metal (e.g. Ca, Sr, Ba ${ }^{59}$. The CMR effect was first observed in 1950 by Jonker and van Santen. There has been a resurgence in interest since 1993 due to the large negative magnetoresistance effect found in thin films ${ }^{60}$. Magnetoresistance values of $99.9 \%$ (when normalised to the zero field value) at $77 \mathrm{~K}$ and in a magnetic field of $6 \mathrm{~T}$ have been reported $\underline{61}$.

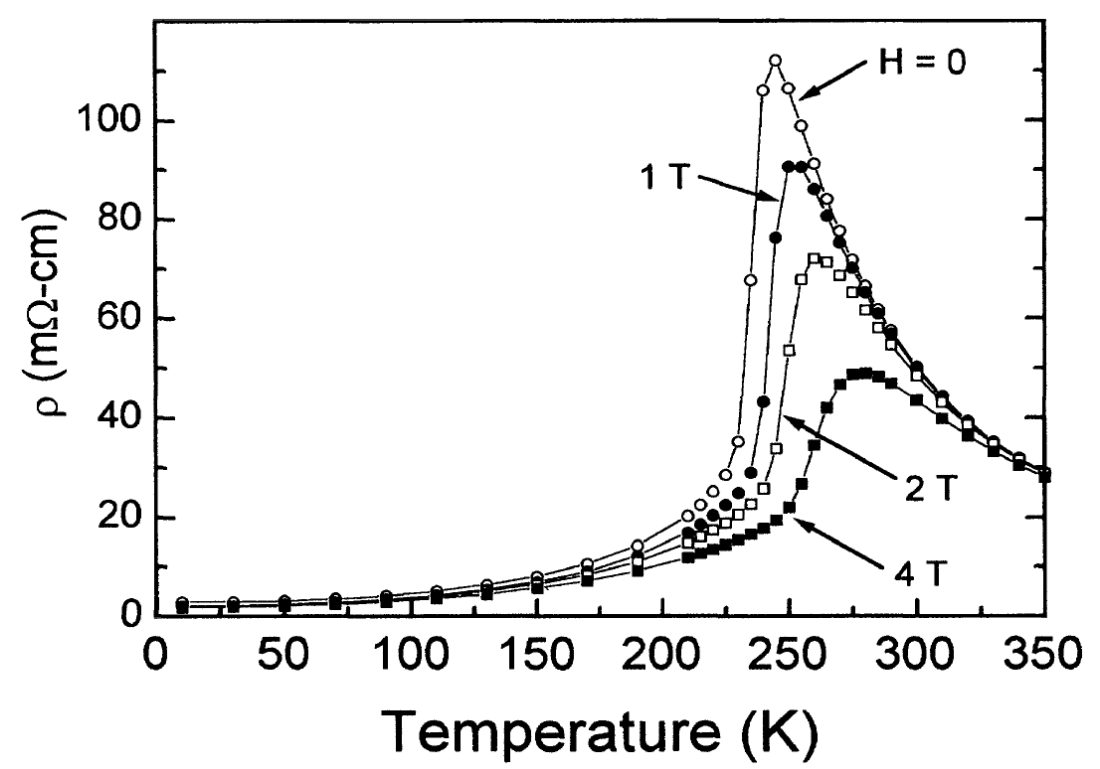

Figure 2.7: Plot of resistivity against temperature for $\mathrm{La}_{0.75} \mathrm{Ca}_{0.25} \mathrm{MnO}_{3}$, at various applied magnetic fields. We see that the resistivity is suppressed with increasing magnetic field. (Adapted from reference [6).

The magnetoresistance effect occurs due to a paramagnetic (insulating) to ferromagnetic (metallic) phase transition. Figure 2.7 shows the resistivity as a function of temperature for various values of magnetic field. Here, the resistivity at zero field shows the phase 
transition from ferromagnetic below the Curie temperature to paramagnetic above. The application of a magnetic field suppresses resistivity and shifts the Curie temperature to a higher temperature, thus the magnetic field causes the switch between the low and high resistance states. This behaviour is usually explained via the double exchange mechanism ${ }^{6}$. Here, the electrical conduction occurs due to indirect hopping of electrons between $\mathrm{Mn}^{3+}$ and $\mathrm{Mn}^{4+}$ ions via the $\mathrm{O}^{2-}$ ion and not from metallic conduction ${ }^{262}$.

\subsubsection{Ballistic Magnetoresistance (BMR)}

Ballistic magnetoresistance (BMR) was a term coined in 1999 when García, Muñoz \& Zhao reported an extremely large magnetoresistance effect of $280 \%$ at room temperature in a magnetic field of $0.01 \mathrm{~T}^{63}$. Subsequently, much larger magnetoresistance values of up to $100,000 \%$ have also been attributed to the BMR effect $\underline{6465}$. The systems in which the BMR effect has been reported are ferromagnetic nanocontacts. Here, a needle and a wire are connected via a nanoscale contact and conduction is of a ballistic nature. This is where the mean free path of electrons is larger than the dimensions of the nanocontact, thus electrons can pass through the device without scattering from the lattice. For a spin-polarised current the spin dependent scattering is more pronounced when the magnetisation state of the nanocontact is antiferromagnetic. Magnetisation of the nanocontacts depends on the locations of the magnetic domains. A plausible model for the shifting of these domains from a ferromagnetic to an antiferromagnetic configuration under an applied magnetic field was published and can be seen in Figure $2.8^{17}$.

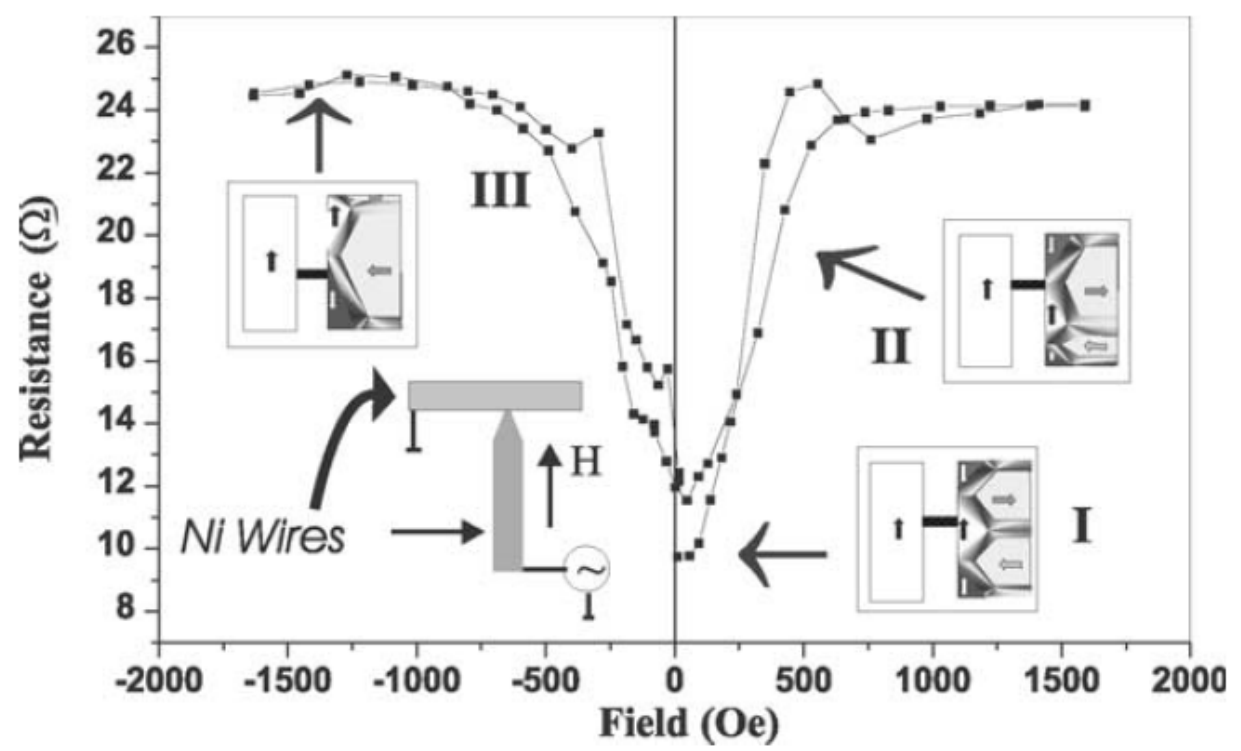

FIGURE 2.8: Magnetoresistance results for a nickel (Ni) nanocontact as a function of magnetic field. The inset diagrams show a plausible model of domain shifting with the application of a magnetic field in an attempt to describe the experimental data.

(Reproduced from reference 7). 
The BMR effect attracted much attention in the years after its discovery due to its potential for a new generation of spintronic devices. However, when trying to reproduce the experimental results several research groups encountered difficulties, raising questions concerning the validity of the BMR effect ${ }^{66}$. In 2004 a consensus emerged from the 'Symposium on the Controversy Over Ballistic Magnetoresistance' criticising reported BMR results due to the lack of stability on the atomic scale causing the nanocontact to be distorted and thereby changing its electrical resistance ${ }^{66 / 67}$. The conclusions of the symposium are listed below.

- "Many previous attempts to observe a BMR effect have been subject to serious artifacts that can mimic BMR.

- Experiments carefully designed to avoid these artifacts do not provide evidence for a real BMR effect.

- It is entirely possible that there is no real BMR effect of any significant magnitude in any previously published data. " 68

\subsubsection{Geometric Magnetoresistance}

The geometrical magnetoresistance effect is a special case of the Hall effect where the geometry of the system acts to short circuit the Hall voltage ${ }^{69}$. The magnetoresistance arises as a result of the Lorentz force acting on the charge carriers, influencing the current flow and potential distribution in the system. Consider a rectangular slab of semiconducting material of length $\mathrm{L}$ and width $\mathrm{W}$, as seen in Figure 2.9.

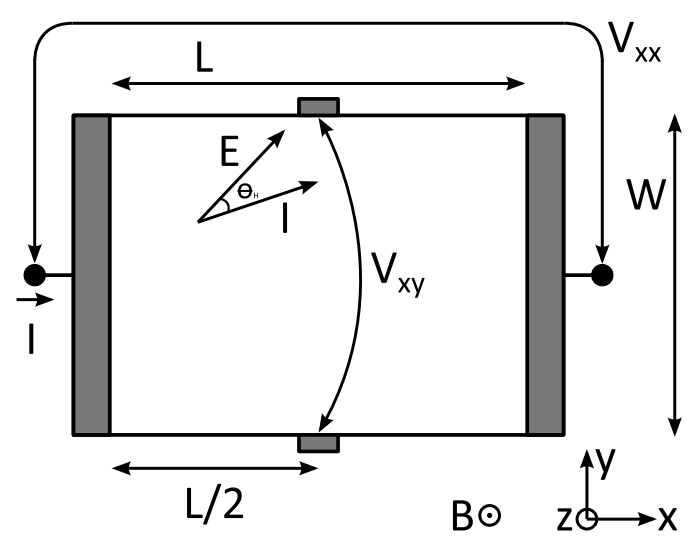

Figure 2.9: A rectangular semiconducting slab of length $\mathrm{L}$ and width $\mathrm{W}$ with a current (I) forced to flow between the left and right contacts. A magnetic field (B) is applied along the $\mathrm{z}$ axis, with the resultant Lorentz force creating a Hall angle $\left(\theta_{\mathrm{H}}\right)$ to arise between the electric field (E) and the current (I). Geometrical magnetoresistance arises for such a system with $\mathrm{L} \leq \mathrm{W}$. (Adapted from reference $[$ ). 
Here, the application of a voltage $\mathrm{V}_{x x}$ forces current (I) to flow through the system, with a magnetic field (B) applied in the $\mathrm{z}$ direction. The Lorentz force deflects the current flow towards one face of the slab creating a charge separation in the y direction. This establishes a transverse electric (Hall) field and therefore the Hall voltage $\left(\mathrm{V}_{x y}\right)$. For a system with $\mathrm{L} \gg \mathrm{W}$, the Lorentz force and the Hall field balance and the current flows in the direction of the $\mathrm{x}$ axis. In this case (Hall effect) there is no geometrical magnetoresistance and any magnetoresistive response would be attributed to the presence of a physical OMR effect. However, for a system where $\mathrm{L} \leq \mathrm{W}$, the Hall field is shorted out by the contacts. The Lorentz force is no longer compensated for and the current flows at an angle $\left(\theta_{\mathrm{H}}\right)$ relative to the electric field. This results in an increase in the path length of the current flow and therefore the system resistance. This geometrical magnetoresistance effect is proportional to $\mathrm{B}^{2}$ at small magnetic fields ${ }^{70}$. Conformal mapping can show that a Corbino disk geometry is equivalent to an infinitely wide or short rectangular element. In this case the Hall voltage is shorted completely and the geometrical magnetoresistance is maximal ${ }^{8}$. In GaAs the geometrical magnetoresistance was found to reach $50 \%$ in a magnetic field of $1 \mathrm{~T}$ at room temperature ${ }^{70}$.

\subsubsection{Extraordinary Magnetoresistance (EMR)}

Extraordinary magnetoresistance (EMR) was discovered in 2000 by Solin et al. following research into the properties of a semiconductor superlattice and is the basis of the majority of work presented in this Thesis ${ }^{914}$. EMR is an enhanced geometric magnetoresistance effect where the semiconductor-metal hybrid systems result in magnetoresistance values orders of magnitude larger than any previously discovered effects. Extremely large magnetoresistance values of $100 \%, 750,000 \%$ and over 1,000,000\% have been observed at room temperature in applied magnetic fields of $0.05 \mathrm{~T}, 4 \mathrm{~T}$ and $5 \mathrm{~T}$ respectively ${ }^{9}$. The effect was discovered in non-magnetic semiconductor-metal hybrids where a circular metallic $(\mathrm{Au})$ disk (of radius $r_{a}$ ) is embedded concentrically inside a larger semiconducting (InSb) disk (of radius $r_{b}$ ). The system contains four contacts placed equidistant around the perimeter of the semiconducting disk; two of which input and output the current while the remaining two measure the potential difference. This geometry is seen in Figure 2.10. The filling factor $(\alpha)$ can be defined in order to quantify the relative proportion of the system that is metallic as opposed to semiconducting,

$$
\alpha=\frac{r_{a}}{r_{b}}=\sqrt{\frac{\text { Area of metallic region }}{\text { Total system area }}} .
$$

The EMR effect has generated significant interest since its initial discovery. The large number of potential practical applications, in particular, the improvement to future read 


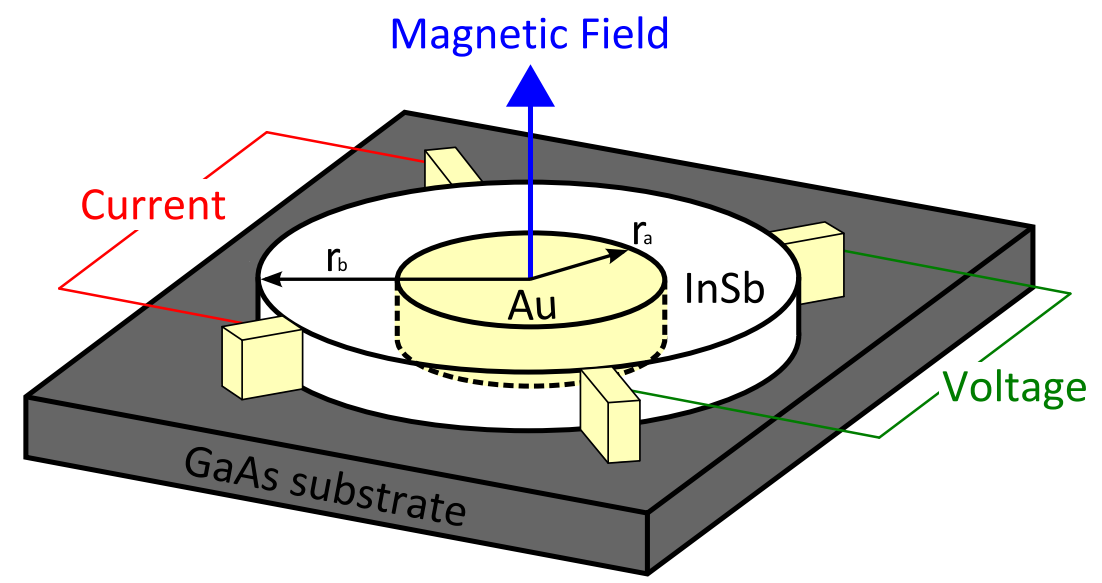

Figure 2.10: A circular geometry EMR device containing an outer disk of semiconducting material ( $\mathrm{InSb}$ of radius $r_{b}$ ) and an inner embedded metallic region (Au of radius $r_{a}$ ). Four contacts are situated equidistant around the disk perimeter, two contacts input and output the current while the other two measure the potential difference. The magnetic field is applied perpendicular to the plane of the $\operatorname{disk}^{9}$.

heads of hard disk drives, is a key driving factor of such attention $\underline{1471}$ 173. Here, magnetic field sensors that can detect small changes in magnetic field at room temperature are required. Current hard disk drives have a data storage density of 20 gigabits per square inch $\left(\mathrm{Gb} / \mathrm{in}^{2}\right)$; future hard drives strive to reach densities of $1,000 \mathrm{~Gb} / \mathrm{in}^{2}$. In order to achieve this the read heads of such devices need to be smaller in size whilst still producing a significant magnetoresistance effect in small magnetic fields (of the order of $0.05 \mathrm{~T}$ ). Existing read heads utilise the GMR effect, however such devices made from magnetic materials may be limited by magnetic noise when they are miniaturised. The EMR effect, similar to ordinary and geometric magnetoresistance and unlike all other previously discovered magnetoresistance effects, arises in systems made from exclusively non-magnetic materials thus giving the potential for a much larger signal to noise ratio 14 .

The EMR effect arises from the current redistribution in the system when a magnetic field is applied. The conductivity of gold is over 2,000 times that of the semiconducting indium antimonide. In zero or small magnetic fields the electric field lines are perpendicular to the boundary of the conducting disk with the electric current flowing along these lines (zero Hall angle). Therefore, the majority of the current flows through the metallic region, creating a short circuit (low resistance path). However, the application of a magnetic field perpendicular to the surface of the disk causes the Hall angle between the electric field lines and the current flow to become non-zero, approaching $90^{\circ}$ for large fields. Consequently, the current flow becomes tangential to the metallic surface and current is forced to flow through the semiconductor; around the metallic region as if it were not present 14 . This creates an open circuit (high resistance path) resulting in huge values of magnetoresistance. Figure 2.11 illustrates how this mechanism gives rise to two 
distinct situations with significantly different resistances. A more in-depth review of the EMR effect is provided in Chapter 3.

\section{Low Resistance $(B=0)$}

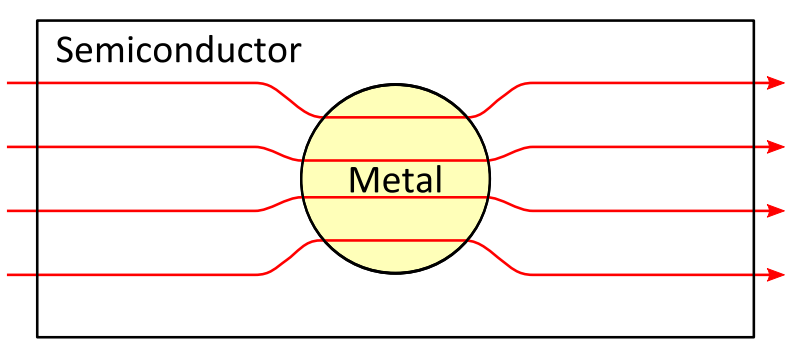

Electric Current Flow

\section{High Resistance $(\mathrm{B}>0)$}

$\odot$ Magnetic Field

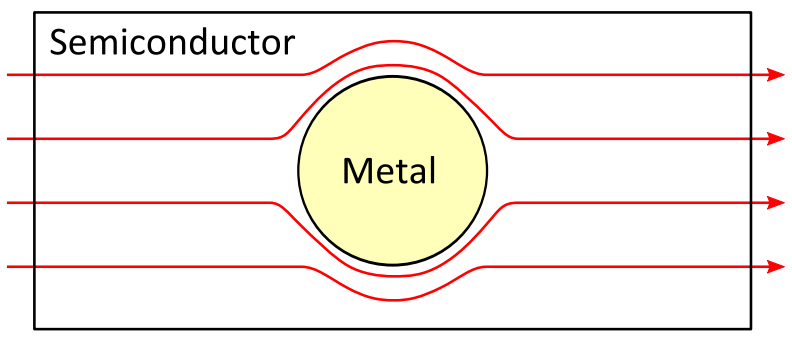

Electric Current Flow

FIGURE 2.11: Visualisation of the underlying principle responsible for the EMR effect. A low resistance regime is produced without magnetic field, with the application of a magnetic field producing a high resistance state. The current redistribution between these two states arises due to the Hall angle approaching $90^{\circ}$ for large magnetic fields.

\subsection{Hall Effect}

The Hall effect, discovered in 1879 by Hall ${ }^{74}$, considers a system where a current carrying conductor is subjected to a magnetic field perpendicular to the direction of the current flow* The charge carriers themselves experience a force deflecting their motion to one side of the conductor, namely the Lorentz force (see Figure 2.12 75

$$
\mathbf{F}_{L}=-e(\mathbf{v} \times \mathbf{B}) .
$$

${ }^{*}$ By convention the direction of current flow is opposite to that of electrons 
Here, $\mathbf{B}$ is the applied magnetic field ( $\mathbf{z}$ direction), $\mathbf{v}$ the velocity of the charge carriers and $\mathrm{e}$ is the elementary charge $\left(e=1.6 \times 10^{-19} \mathrm{C}\right)$. The effect of the deflection of the charge carriers in the sample is the creation of a surface charge density, due to the excess charge build-up on one side of the conductor compared to the other. This means a transverse electric field is established in the y direction, namely the Hall field $\left(E_{y}\right)$. This produces the Hall voltage $\left(V_{\mathrm{H}}\right)$, measured across faces of the sample in the $\mathrm{y}$ direction. The Hall voltage takes the same sign as the charge of the charge carriers; negative for electrons and positive for holes. In equilibrium the Hall field balances the Lorentz force on the charge carriers and current flows only in the $\mathrm{x}$ direction ${ }^{75}$.

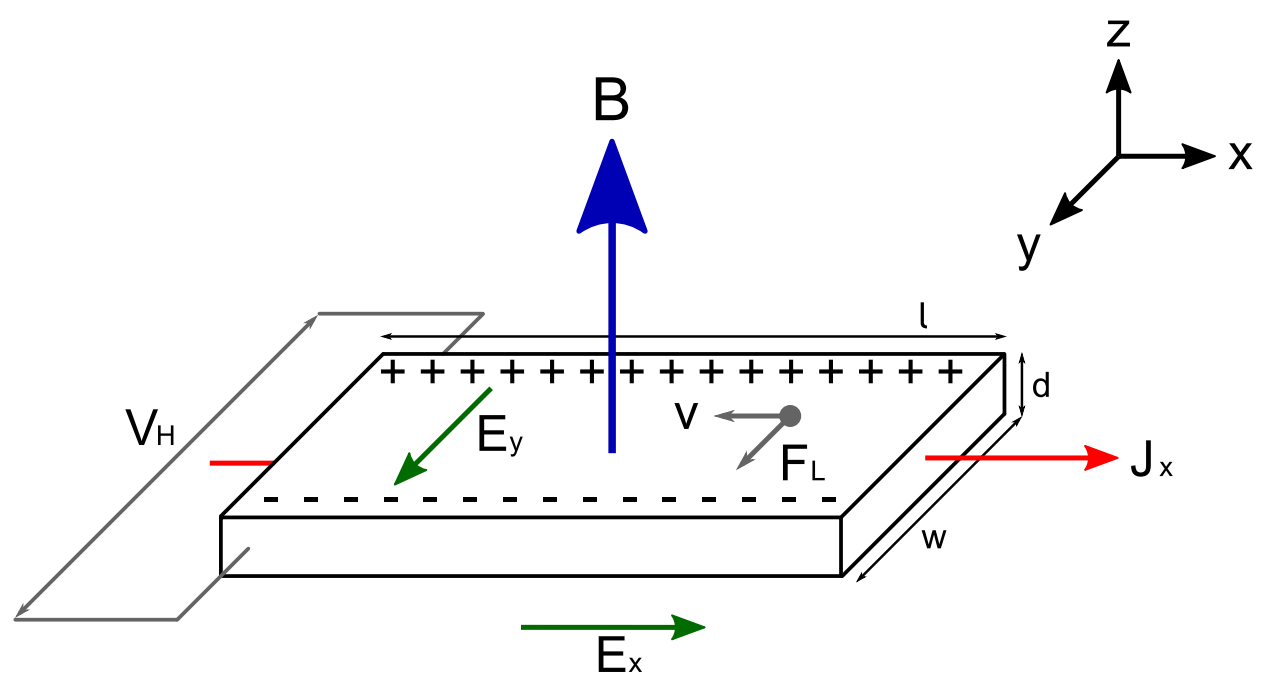

FIGURE 2.12: The Hall effect: here $w$ and $d$ are the width and depth of the sample respectively $(A=w \times d) ; l$ is the length of the sample; $V_{\mathrm{H}}$ is the Hall voltage; $E_{x}$ is the applied electric field producing $J_{x}$ the current density $(J=I / A) ; E_{y}$ is the transverse Hall electric field; $v$ is the velocity of the charge carriers; and $F_{L}$ is the Lorentz force. In this illustration the charge carriers are assumed to be negatively charged (electrons).

The total force on the charge carrier is then a combination of the Lorentz force (magnetic) and the electric force (created by the presence of the electric field) which can be seen in the following expression

$$
\mathbf{F}=-e(\mathbf{E}+\mathbf{v} \times \mathbf{B})
$$

With the magnetic field directed along the $\mathrm{z}$ axis, the current driven along the $\mathrm{x}$ axis, and the electric field in the plane of the solid, we have 


$$
\begin{gathered}
\mathbf{B}=\left(\begin{array}{c}
0 \\
0 \\
B_{z}
\end{array}\right), \\
\mathbf{E}=\left(\begin{array}{c}
E_{x} \\
E_{y} \\
0
\end{array}\right), \\
\mathbf{J}=\left(\begin{array}{c}
J_{x} \\
0 \\
0
\end{array}\right) .
\end{gathered}
$$

In general, the angle the electric field $(\mathbf{E})$ makes with the current density $(\mathbf{J})$ is called the Hall angle $\left(\theta_{\mathrm{H}}\right)$. This can be expressed as follows in Equation $2.8^{29}$

$$
\tan \theta_{\mathrm{H}}=\mu B_{z}=\omega_{c} \tau
$$

Another important parameter is the Hall coefficient $\left(R_{\mathrm{H}}\right)^{76}$

$$
R_{\mathrm{H}}=\frac{E y}{J x B_{z}}=-\frac{1}{n e}
$$

Similarly to the Hall voltage, the Hall coefficient is negative for electrons (as in Equation 2.9p and positive for holes. Therefore, the Hall effect can be used to determine the charge and number of charge carriers present in a sample, from which other material specific parameters can be calculated (such as the carriers drift velocity). Equation 2.9 is determined using the Drude theory of conductivity and suggests that the Hall coefficient is independent of both applied magnetic field and temperature. Experimentally this is not always the case. The Hall coefficient of a sample can be measured using the van der Pauw method (see Appendix A.

The quantum Hall effect (QHE) is a quantum manifestation of the Hall effect that generally occurs in two-dimensional electron systems, at low temperatures, in the presence of strong magnetic fields. Under these conditions the Hall conductivity is quantised, exhibiting plateaus at multiples of $e^{2} / h$

$$
\sigma_{x y}=\frac{\nu e^{2}}{h} .
$$


Here, $\mathrm{h}$ is Planck's constant, e is the elementary charge and $\nu$ can either be an integer (integer quantum Hall effect, IQHE) or a simple rational fraction (fractional quantum Hall effect, FQHE). The quantisation of the Hall effect was realised by Klaus von Klitzing in 1980 for which he was awarded the 1985 Nobel Prize in Physics 77.

\subsection{Electron Transport Without a Magnetic Field}

In order to describe the motion of electrons in an external electric field we use the classical equation of motion*

$$
m^{*}\left(\frac{d^{2} \mathbf{r}}{d t^{2}}+\frac{1}{\tau} \frac{d \mathbf{r}}{d t}\right)=-e \mathbf{E}
$$

The force acting in this case is equal to $-e \mathbf{E}$, e is the elementary charge $\left(e=1.6 \times 10^{-19} \mathrm{C}\right)$, $\mathbf{E}$ is the applied electric field, $m^{*}$ is the effective mass of the electron, $\mathbf{r}$ is the electron position and $\tau$ is the relaxation time (average time between scattering events). In a

steady state (acceleration of electrons equal to zero) the electron velocity $\left(\mathbf{v}=\frac{d \mathbf{r}}{d t}\right)$ is known as the drift velocity of electrons $\left(\mathbf{v}_{d}\right)$

$$
\mathbf{v}_{d}=\frac{-e \mathbf{E} \tau}{m^{*}} .
$$

As is convention, the direction of motion of the electrons is opposite to that of the electric field. The current density $(\mathbf{J}=I / A)$ is related to the drift velocity as follows

$$
\mathbf{J}=-n e \mathbf{v}_{d}
$$

Here, $\mathrm{n}$ is the carrier concentration (number of charge carriers per unit volume). Combining Equations 2.12 and 2.13 gives

$$
\mathbf{J}=\frac{n e^{2} \tau}{m^{*}} \mathbf{E}
$$

From the following expression of Ohm's Law we can define the conductivity tensor $(\hat{\sigma})$

$$
\mathbf{J}=\hat{\sigma} \mathbf{E}
$$

\footnotetext{
${ }^{*}$ The work in this section follows that contained in the book Fundamentals of Semiconductors ${ }^{78}$
} 
where

$$
\hat{\sigma}=\left(\begin{array}{ccc}
\sigma_{x x} & \sigma_{x y} & \sigma_{x z} \\
\sigma_{y x} & \sigma_{y y} & \sigma_{y z} \\
\sigma_{z x} & \sigma_{z y} & \sigma_{z z}
\end{array}\right)=\left(\begin{array}{ccc}
\sigma_{0} & 0 & 0 \\
0 & \sigma_{0} & 0 \\
0 & 0 & \sigma_{0}
\end{array}\right) .
$$

Therefore the zero field conductivity is given by

$$
\sigma_{0}=\frac{n e^{2} \tau}{m^{*}}
$$

The carrier mobility, $\mu$, is defined by

$$
\mathbf{v}_{d}=\mu \mathbf{E}
$$

From Equation 2.12 we obtain

$$
\mathbf{v}_{d}=-\frac{e \tau}{m^{*}} \mathbf{E}=-\mu \mathbf{E}
$$

Therefore the mobility of electrons is

$$
\mu=\frac{e \tau}{m^{*}}
$$

In a semiconductor containing both free electrons and holes the zero field conductivity can be defined as

$$
\sigma_{0}=e\left(n_{e} \mu_{e}+n_{h} \mu_{h}\right) .
$$

Where $n_{e}$ and $n_{h}$ are the carrier concentrations of the electrons and holes and $\mu_{e}$ and $\mu_{h}$ are the mobilities of electrons and holes respectively. Since the resistivity is the inverse of the conductivity the zero field resistivity $\left(\rho_{0}\right)$ is given as

$$
\rho_{0}=\frac{1}{\sigma_{0}}=\frac{m^{*}}{n e^{2} \tau} .
$$




\subsection{Electron Transport With a Magnetic Field}

The application of a magnetic field (in the $\mathrm{z}$ direction) along with an external electric field (as in Section 2.3) gives rise to the following equation of motion for electrons:

$$
m^{*}\left(\frac{d^{2} \mathbf{r}}{d t^{2}}+\frac{1}{\tau} \frac{d \mathbf{r}}{d t}\right)=-e[\mathbf{E}+(\mathbf{v} \times \mathbf{B})]
$$

Here, the Lorentz force now also acts on the charge carrier along with the force exerted by the presence of the electric field, as in the previous section. In a steady state (acceleration of electrons equal to zero) the electron velocity $\left(\mathbf{v}=\frac{d \mathbf{r}}{d t}\right)$ is known as the drift velocity of electrons $\left(\mathbf{v}_{d}\right)$ giving

$$
\left(\frac{m^{*}}{\tau}\right) \mathbf{v}_{d}=-e\left[\mathbf{E}+\left(\mathbf{v}_{d} \times \mathbf{B}\right)\right]
$$

This can be written in terms of its components along the $\mathrm{x}, \mathrm{y}$ and $\mathrm{z}$ axes

$$
\begin{gathered}
\left(\frac{m^{*}}{\tau}\right) \mathbf{v}_{d x}=-e\left[E_{x}+\left(v_{d y} B_{z}\right)\right], \\
\left(\frac{m^{*}}{\tau}\right) \mathbf{v}_{d y}=-e\left[E_{y}+\left(v_{d x} B_{z}\right)\right], \\
\left(\frac{m^{*}}{\tau}\right) \mathbf{v}_{d z}=-e E_{z} .
\end{gathered}
$$

From Equation 2.13 we can obtain expressions for the current density by multiplying these equations by $-n e$

$$
\begin{gathered}
J_{x}=\left(\frac{n e^{2} \tau}{m^{*}}\right) E_{x}-\left(\frac{e B_{z}}{m^{*}}\right) \tau J_{y}, \\
J_{y}=\left(\frac{n e^{2} \tau}{m^{*}}\right) E_{y}+\left(\frac{e B_{z}}{m^{*}}\right) \tau J_{x}, \\
J_{z}=\left(\frac{n e^{2} \tau}{m^{*}}\right) E_{z} .
\end{gathered}
$$

\footnotetext{
${ }^{*}$ The work in this section follows that contained in the books Fundamentals of Semiconductors ${ }^{78}$ and Physics of Semiconductors in High Magnetic Fields ${ }^{[79}$
} 
The classical cyclotron frequency $\left(\omega_{c}\right)$ can now be introduced as

$$
\omega_{c}=\frac{e B_{z}}{m^{*}} .
$$

Given Equations 2.17 and 2.31 we can write

$$
\begin{gathered}
J_{x}=\sigma_{0} E_{x}-\omega_{c} \tau J_{y}, \\
J_{y}=\sigma_{0} E_{y}+\omega_{c} \tau J_{x}, \\
J_{z}=\sigma_{0} E_{z} .
\end{gathered}
$$

Solving these equations results in the three components of the current density:

$$
\begin{gathered}
J_{x}=\frac{1}{1+\left(\omega_{c} \tau\right)^{2}} \sigma_{0}\left(E_{x}-\omega_{c} \tau E_{y}\right), \\
J_{y}=\frac{1}{1+\left(\omega_{c} \tau\right)^{2}} \sigma_{0}\left(E_{y}+\omega_{c} \tau E_{x}\right), \\
J_{z}=\sigma_{0} E_{z} .
\end{gathered}
$$

We can then define a generalised magneto-conductivity tensor, $\hat{\sigma}(\mathbf{B})$, as

$$
\hat{\sigma}(\mathbf{B})=\left(\begin{array}{ccc}
\sigma_{x x} & \sigma_{x y} & \sigma_{x z} \\
\sigma_{y x} & \sigma_{y y} & \sigma_{y z} \\
\sigma_{z x} & \sigma_{z y} & \sigma_{z z}
\end{array}\right)=\frac{\sigma_{0}}{1+\left(\omega_{c} \tau\right)^{2}}\left(\begin{array}{ccc}
1 & -\omega_{c} \tau & 0 \\
\omega_{c} \tau & 1 & 0 \\
0 & 0 & 1+\left(\omega_{c} \tau\right)^{2}
\end{array}\right) .
$$

In a magnetic field the conductivity tensor has non-diagonal components due to the presence of the Lorentz force acting on the charge carriers. By defining the parameter $\beta$ (the dimensionless magnetic field) as

$$
\beta=\mu B_{z}=\frac{e B_{z} \tau}{m^{*}}=\omega_{c} \tau
$$

We can write 


$$
\hat{\sigma}(\mathbf{B})=\frac{\sigma_{0}}{1+\beta^{2}}\left(\begin{array}{ccc}
1 & -\beta & 0 \\
\beta & 1 & 0 \\
0 & 0 & 1+\beta^{2}
\end{array}\right) .
$$

In two-dimensions this reduces to

$$
\hat{\sigma}(\mathbf{B})=\frac{\sigma_{0}}{1+\beta^{2}}\left(\begin{array}{cc}
1 & -\beta \\
\beta & 1
\end{array}\right) .
$$

When the magnetic field equals zero we see that Equation 2.40 reduces to Equation 2.16 in the previous section. The resistivity tensor is the inverse of the conductivity tensor and is defined by

$$
\mathbf{E}=\hat{\rho} \mathbf{J} .
$$

Therefore, the resistivity tensor is:

$$
\hat{\rho}(\mathbf{B})=\rho_{0}\left(\begin{array}{ccc}
1 & \beta & 0 \\
-\beta & 1 & 0 \\
0 & 0 & 1
\end{array}\right) .
$$

This is the resistivity that is measured experimentally. The van der Pauw method for measuring the resistivity of a thin film can be used to measure the magnetoresistance of a sample. This method was used in the experiments of Solin et al. and is discussed in more detail in Appendix $A^{9}$.

\subsection{Finite Element Method and Modelling}

The finite element method (FEM) is a numerical technique for obtaining approximate solutions to problems described by partial differential equations (PDEs) that are difficult or impossible to solve analytically ${ }^{80}$. Finite element analysis essentially converts a continuous problem into a discrete problem that can be solved ${ }^{81}$. This is achieved by dividing a system's domain into many discrete elements of a finite size comprised of interconnecting nodes (discretisation). By determining the behaviour of each individual element (a system of linear equations is produced) and assembling their effects (the nodes

\footnotetext{
${ }^{*}$ The free electron model does not include the physical component of the magnetoresistance
} 
are coupled similar to that of a spring-mass system) a solution for the entire domain can be obtained 82 . Increasing the number of elements in a domain improves the solution of the model at the cost of an increased solution time. Since two-dimensional models require much fewer elements than three-dimensional models it is often desirable to reduce the problem to two-dimensions when possible. The great advantages of the FEM are the ability to model complex system geometries and to solve problems covering more than one area of Physics (multiphysics problems).

The FEM simulations in this work were performed utilising the Comsol Multiphysics software package (Version 3.4). There are many things to be considered when simulating a physical system using Comsol Multiphysics. The main points considered during simulations are outlined below.

\section{Selecting an appropriate application module}

The first thing to consider when using Comsol Multiphysics is the choice of module, application mode and model dimension. Comsol Multiphysics allows for twoor three-dimensional models to be created. The advantages of modelling in twodimensions are a reduction in both computation time and computing power required in order to achieve a solution to the problem. The choice of application mode is based on the underlying PDE to be solved in the model (if none of the built-in application modes are suitable, any PDE can be input manually). For multiphysics problems multiple application modes can be combined. For the simulations in this Thesis the conductive media DC application mode inside the AC/DC module was used due to its ability to handle electrostatic problems. The conductive media DC application mode is based on the following equations ${ }^{83}$. Firstly, consider the point form of Ohm's Law and the equation of continuity, where $\mathbf{J}^{e}$ is an externally generated current density

$$
\begin{gathered}
\mathbf{J}=\sigma \mathbf{E}+\mathbf{J}^{e}, \\
\nabla \cdot \mathbf{J}=0 .
\end{gathered}
$$

By combining Equations 2.44 and 2.45 we get

$$
\nabla \cdot \mathbf{J}=\nabla \cdot\left(\sigma \mathbf{E}+\mathbf{J}^{e}\right)=0
$$

Since, $\mathbf{E}=-\nabla V$, we obtain

$$
\nabla \cdot \mathbf{J}=-\nabla \cdot\left(\sigma \nabla V-\mathbf{J}^{e}\right)=0
$$


This expression can then be generalised for current sources $\left(Q_{j}\right)$, giving Equation 2.48 that is the basis of the conductive media DC application mode ${ }^{83}$

$$
-\nabla \cdot\left(\sigma \nabla V-\mathbf{J}^{e}\right)=Q_{j}
$$

The AC/DC module allows for the electric field, electrostatic potential and the current density (among other parameters) to be output as solutions post-processing.

\section{Creating the desired geometry}

Comsol Multiphysics allows for the physical geometry of the problem to be created directly in the user interface. Alternatively, a specific geometry can be imported directly from a computer-aided design (CAD) file. This geometry includes the boundaries of the system and the point contacts.

\section{Constants defined}

Here, any constants that may be required in later steps in order to fully define the system (that are not already defined as part of the selected application mode) can be input directly or loaded from a previously saved text file. These constants could include values of current, magnetic field or specific material parameters.

\section{Input sub-domain settings}

The parameters defining each sub-domain region of the system are defined at this stage. This can be done by loading material parameters from the Comsol Multiphysics in-built material library. Alternatively, specific material parameters can be manually input allowing greater control. In the conductive media DC application mode the most important property to define for each sub-domain is the electrical conductivity. Comsol Multiphysics gives the ability to define a full anisotropic conductivity tensor (as in Equation 2.38).

\section{Input boundary conditions}

Since the FEM is implemented to provide a solution to PDEs, the system's boundary conditions are required to be specified in order for a single solution to be obtained. Many different types of boundary conditions can be selected in Comsol Multiphisics. The models in this Thesis use two:

- Electric Insulation: $\mathbf{n} \cdot \mathbf{J}=0$.

Here, the current density normal to the boundary is equal to zero, therefore there is no current flowing across the boundary.

- Continuity: $\mathbf{n} \cdot\left(\mathbf{J}_{1}-\mathbf{J}_{2}\right)=0$.

Here, the normal component of the electrical current is continuous across the interior boundary. 


\section{Input point settings}

Specific settings can be defined for certain points in the system. In the conductive media DC application mode this allowed for a point current source $\left(Q_{j}\right)$ to be applied (positive values represent an electric current flowing outwards).

\section{Set mesh parameters}

The FEM requires the discretisation of the system domain. This is achieved in Comsol Multiphysics by creating a mesh which splits the domain into a number of triangular elements connected by nodes. This mesh has a large bearing on the quality of the solution produced. Comsol Multiphysics allows for the mesh density to be controlled by varying certain mesh parameters, such as the overall mesh refinement and the number of elements on a specified boundary. A very fine mesh (with many small elements) produces a solution with high precision but requires a lot of computing power and time to achieve. On the other hand, a coarse mesh (with few large elements) requires less computational power and reaches a solution rapidly but can produce solutions that are unphysical and incorrect. Therefore, a compromise is required in order to obtain a mesh that yields reliable solutions in a sensible time frame with the computing power available. Finding the optimal mesh was an iterative process. A model with the finest mesh possible (for the given computer resources) was solved regardless of the time frame, then the mesh was reduced, resolved and compared. If the two solutions were very similar the reduced mesh was deemed satisfactory.

\section{Solve problem}

Once the separate components of the model were specified, the problem could be solved. In the conductive media DC application mode, solutions of the model result in the electric potential $(\mathrm{V})$. This allowed predefined quantities of current density $(\mathbf{J})$ and electric field (E) to be calculated. The solver speed and computational power required are dependent on the complexity of the model and the refinement of the mesh in the system. In order to run a file for a range of values of a single parameter the parametric solver could be utilised.

\section{Plot solutions}

The post-processing of the solutions could also be handled by Comsol Multiphysics. A range of various output plots are possible including streamline, arrow and surface plots. These outputs can be created into a video file to illustrate the variation of a solution with a change in a specific parameter. Post-processing also allows for values of certain parameters to be recorded at specific points in the system.

In Chapter 3 the details presented above are used to create models of the systems in which the EMR effect was discovered. 


\section{Chapter 3}

\section{Circular Geometry EMR Devices}

In this chapter, experimental EMR results based on systems of a circular geometry (as seen in Figure 2.10) are reviewed. These results provide the basis of the initial work presented in this Thesis. Firstly, the discovery of the EMR effect by Solin et al. is outlined, followed by discussion of later experimental results that use the same materials in the disk's construction $10 \mid 84$. Next, experimental results obtained for systems with the same circular geometry but with different materials are considered. These systems utilise graphene in place of the conventional semiconducting indium antimonide ${ }^{11 \mid 12}$. Finally, such circular geometry EMR devices are modelled using the finite element method. In this instance the mechanism behind the EMR effect was investigated and the magnetoresistance results compared to those found in experiments.

\subsection{Previous Results in a Circular System Geometry}

\subsubsection{Experimental Data}

The experimental discovery of the EMR effect by Solin et al. produced the first results in circular geometry devices (modified van der Pauw disks). The magnetoresistance results from these systems remain the largest EMR values reported in any system to date ${ }^{9}$. The magnetoresistance results, as a function of magnetic field and then filling fraction $(\alpha)$, are presented in Figures 3.2 and 3.3 . Here, the magnetoresistance reaches over 1,000,000\% in a $5 \mathrm{~T}$ applied magnetic field for a disk with a filling fraction of $\alpha=13 / 16$.

The system responsible for the results in Figures 3.2 and 3.3 consisted of a gold $(\mathrm{Au})$ droplet embedded concentrically inside an outer disk made from the high mobility narrow gap semiconductor indium antimonide (InSb). Four electrical contacts, located equidistant around the disks perimeter, allowed for the magnetoresistance measurements 


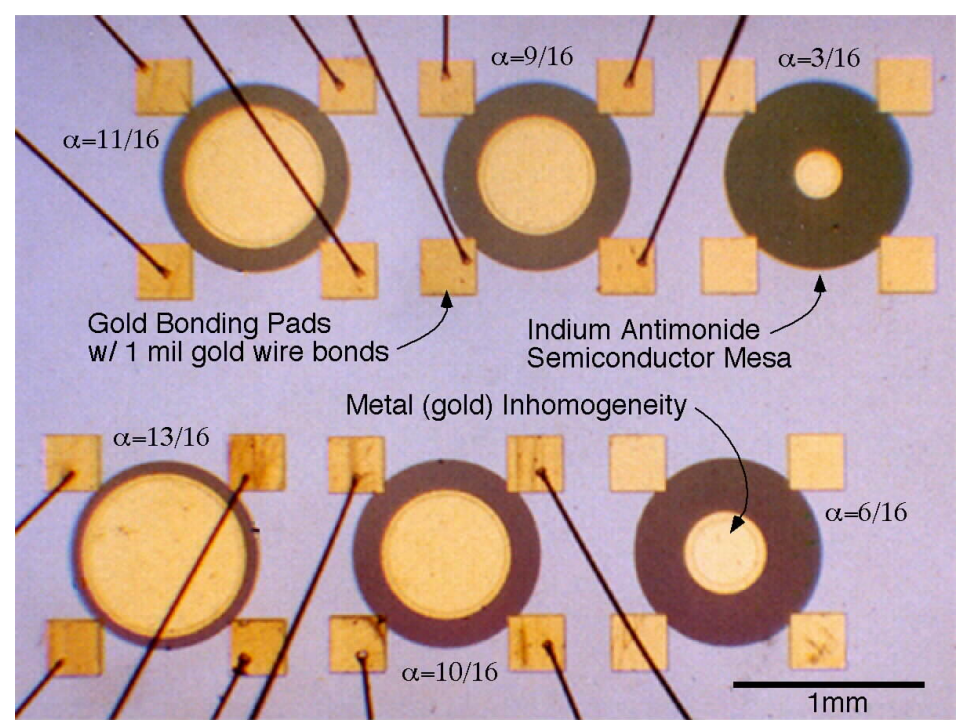

FIGURE 3.1: Six circular geometry experimental EMR devices produced by Solin et $a l$. each with a different value of filling factor $(\alpha)$. Here, the outer semiconducting disk $\left(r_{b}=0.5 \mathrm{~mm}\right)$ contains an embedded metallic inhomogeneity including four electrical $\operatorname{contacts}^{9}$.

to be made using the van der Pauw technique. Here, two of the contacts input and output current while the potential difference is measured across the remaining contacts (see Appendix A for more details). The radius of the semiconducting disk was $0.5 \mathrm{~mm}$ for all of the experimental systems, with the radius of the metallic droplet governed by the value of filling factor with the following values measured: $\alpha=0,2 / 16,3 / 16,4 / 16,6 / 16$, $8 / 16,9 / 16,10 / 16,11 / 16,12 / 16,13 / 16,14 / 16$ and 15/16. The magnetoresistance results were observed at room temperature and in a transverse magnetic field of up to $5 \mathrm{~T}$. The material parameters for these systems (at zero magnetic field and at room temperature) are summarised in Table 3.1.

\begin{tabular}{lccc}
\hline & Conductivity & Mobility & Carrier Conc. \\
& $\sigma(\Omega \mathrm{m})^{-1}$ & $\mu\left(\mathrm{cm}^{2} / \mathrm{Vs}\right)$ & $\mathrm{n}\left(\mathrm{m}^{-3}\right)$ \\
\hline $\mathrm{InSb}$ & $1.86 \times 10^{4}$ & 45,500 & $2.55 \times 10^{22}$ \\
$\mathrm{Au}$ & $4.52 \times 10^{7}$ & 50 & $5.65 \times 10^{28}$ \\
\hline
\end{tabular}

TABLE 3.1: Material parameters of the EMR systems of Solin et al. at zero magnetic field and at room temperature ${ }^{9}$.

The samples consisted of metal organic vapour phase epitaxy-grown epilayers of tellurium (Te) doped n-type InSb. The systems were grown on a 4-inch semi-insulating (100) gallium arsenide (GaAs) substrate $\left(\rho>1 \times 10^{15} \Omega \mathrm{m}\right)$. On this substrate a buffer layer of undoped InSb (thickness 200nm) was grown. The active layer of InSb (thickness $1.3 \mu \mathrm{m}$ ) was deposited on top. The active layer was then capped by a $50 \mu \mathrm{m}$ InSb contacting layer. Finally, this whole sequence was passivated by a $200 \mathrm{~nm}$ layer of silicon nitride $\left(\mathrm{Si}_{3} \mathrm{~N}_{4}\right)$. The buffer layer and thin capping layer do not present parallel conduction channels. The 


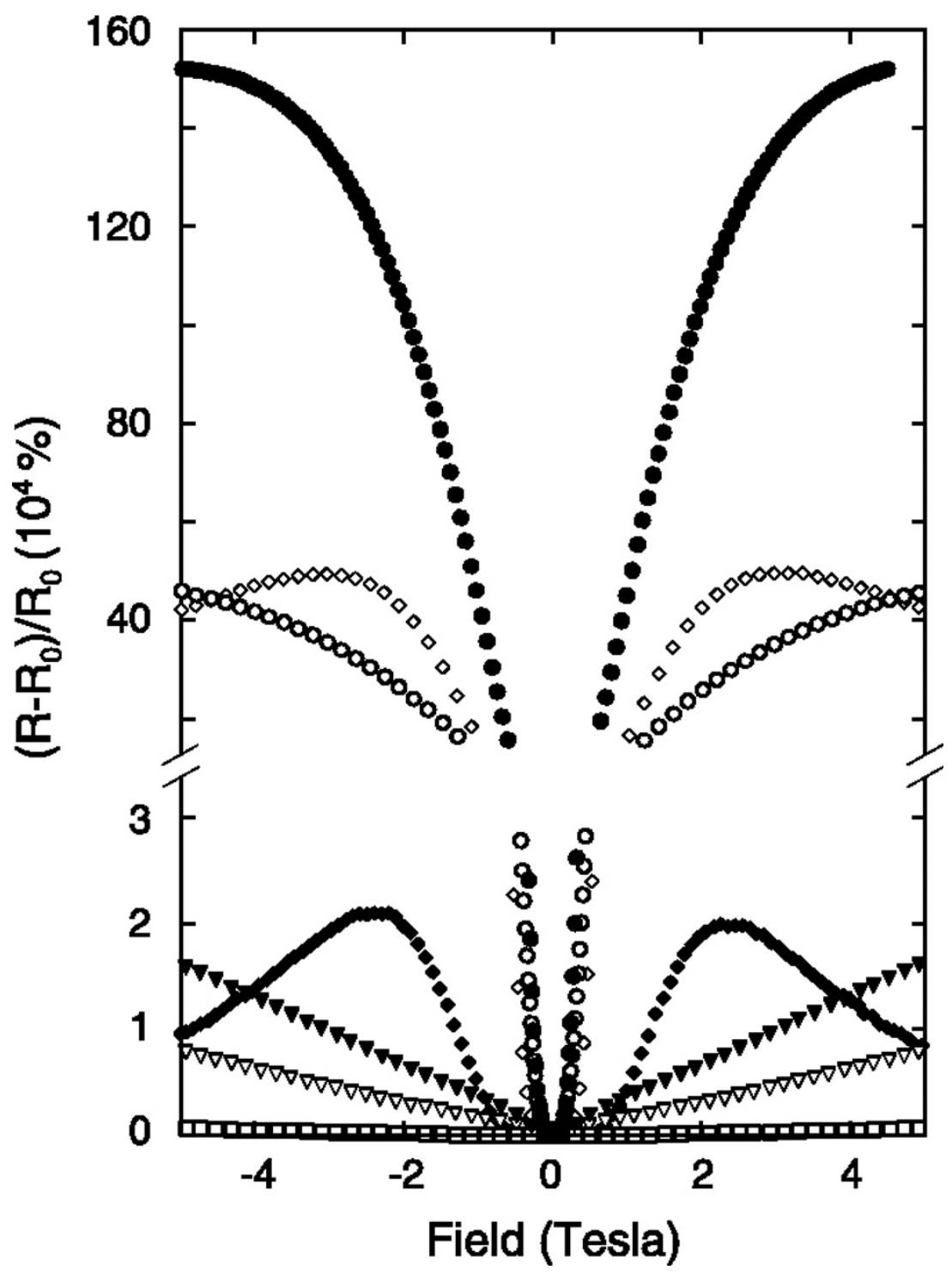

Figure 3.2: Experimental magnetoresistance data produced by Solin et al., where the magnetoresistance is plotted as a function of applied magnetic field for various values of filling factor $(\alpha=0 / 16 \square, 8 / 16 \nabla, 9 / 16 \nabla, 12 / 16 \bigcirc, 13 / 16 \bullet, 14 / 16 \diamond, 15 / 16 \diamond)$.

(Reproduced from reference 9).

wafers were photolithographically patterned into disks with diameter of $1 \mathrm{~mm}$. The disks contained four contact pads and a concentric hole created by reactive ion etching. The side walls of the hole were noted as being smooth but tapered outwards by an average angle of $19^{\circ}$. The hole and contact pads were metallised with a $\mathrm{Ti} / \mathrm{Pt} / \mathrm{Au}$ stack (with gold being the dominant component) thus forming the modified van der Pauw disk that is illustrated in Figure 2.10. The contacts were created by wire bonding 9 . Figure 3.1 shows six typical systems produced in this way each with varying values of filling factor.

The magnetoresistance as a function of magnetic field for these devices can be seen in Figure 3.2. Here, the huge magnitude of the effect is apparent with the magnetoresistance reaching a maximum of $113 \%$ at $0.05 \mathrm{~T}$ and over $1,000,000 \%$ at $5 \mathrm{~T}$. For all values of 


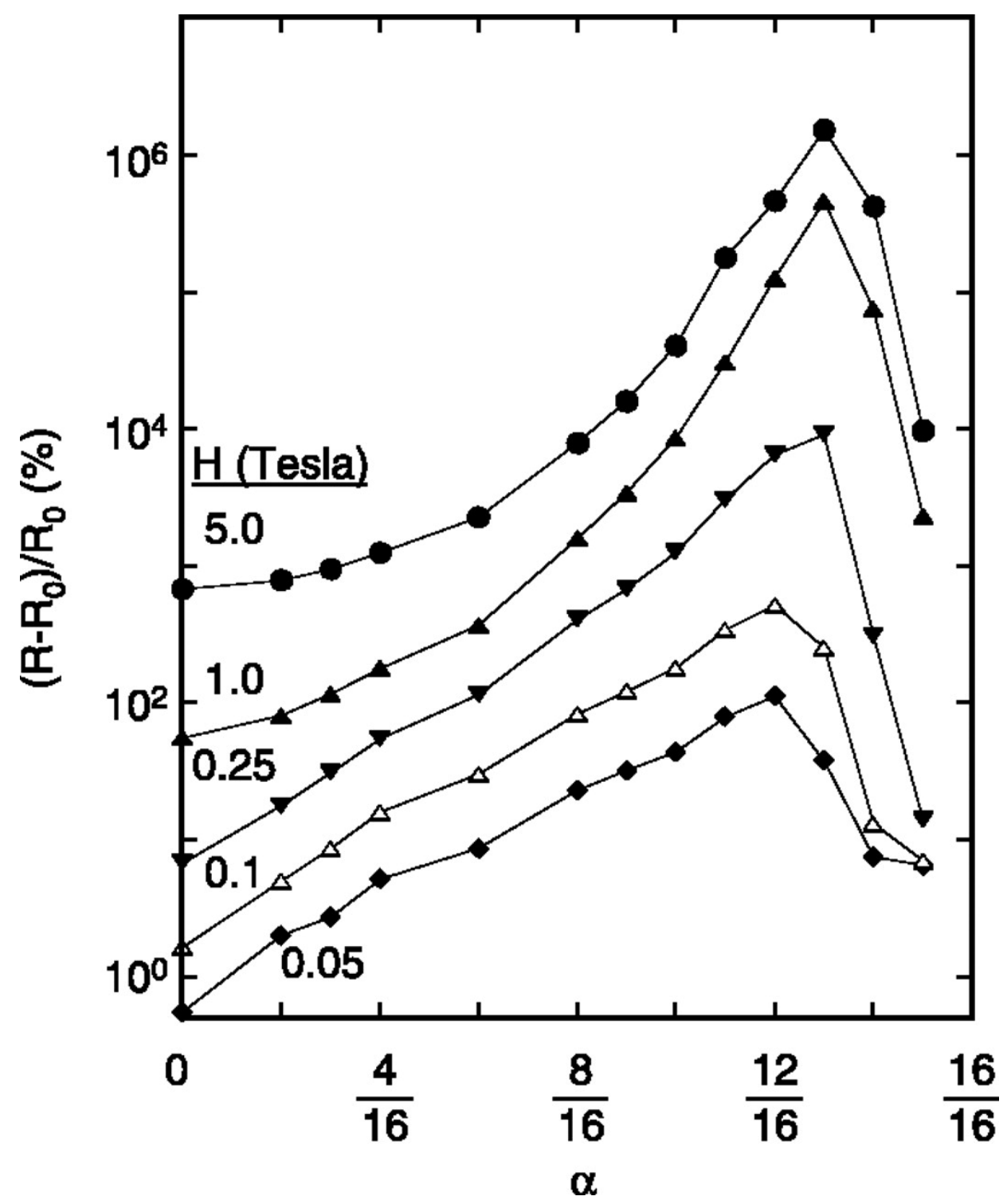

FiguRE 3.3: Experimental magnetoresistance data produced by Solin et al., where the magnetoresistance is plotted as a function of filling factor $(\alpha)$ in various applied magnetic fields $(\mathrm{H}=0.05 \mathrm{~T} \bullet, 0.1 \mathrm{~T} \triangle, 0.25 \mathrm{~T} \boldsymbol{\nabla}, 1.0 \mathrm{~T} \boldsymbol{\Delta}$ and $5 \mathrm{~T} \boldsymbol{\bullet})$. (Reproduced from reference [9).

filling factor of 13/16 and below we see a monotonic increase in the magnetoresistance with magnetic field. However, the two values of filling factor above 13/16 do not follow this pattern and instead peak at a field of approximately $2 \mathrm{~T}$ with the magnitude of the magnetoresistance effect smaller than that found for $\alpha=13 / 16$. The magnetoresistance shows signs of saturation at large magnetic fields especially for the curve with filling factor 13/16. It is apparent the filling factor of the system has a strong influence on the resultant magnetoresistance. This can be seen more clearly in Figure 3.3 where the magnetoresistance is plotted as a function of filling factor for five values of magnetic field. Generally, the magnetoresistance increases for larger values of filling factor until the magnetoresistance peaks. Here, the magnetoresistance peaks for filling factors of 
$12 / 16$ at magnetic fields of $0.05 \mathrm{~T}$ and $0.1 \mathrm{~T}$ (low fields) and 13/16 at magnetic fields of $0.25 \mathrm{~T}, 1 \mathrm{~T}$ and $5 \mathrm{~T}$ (high fields).

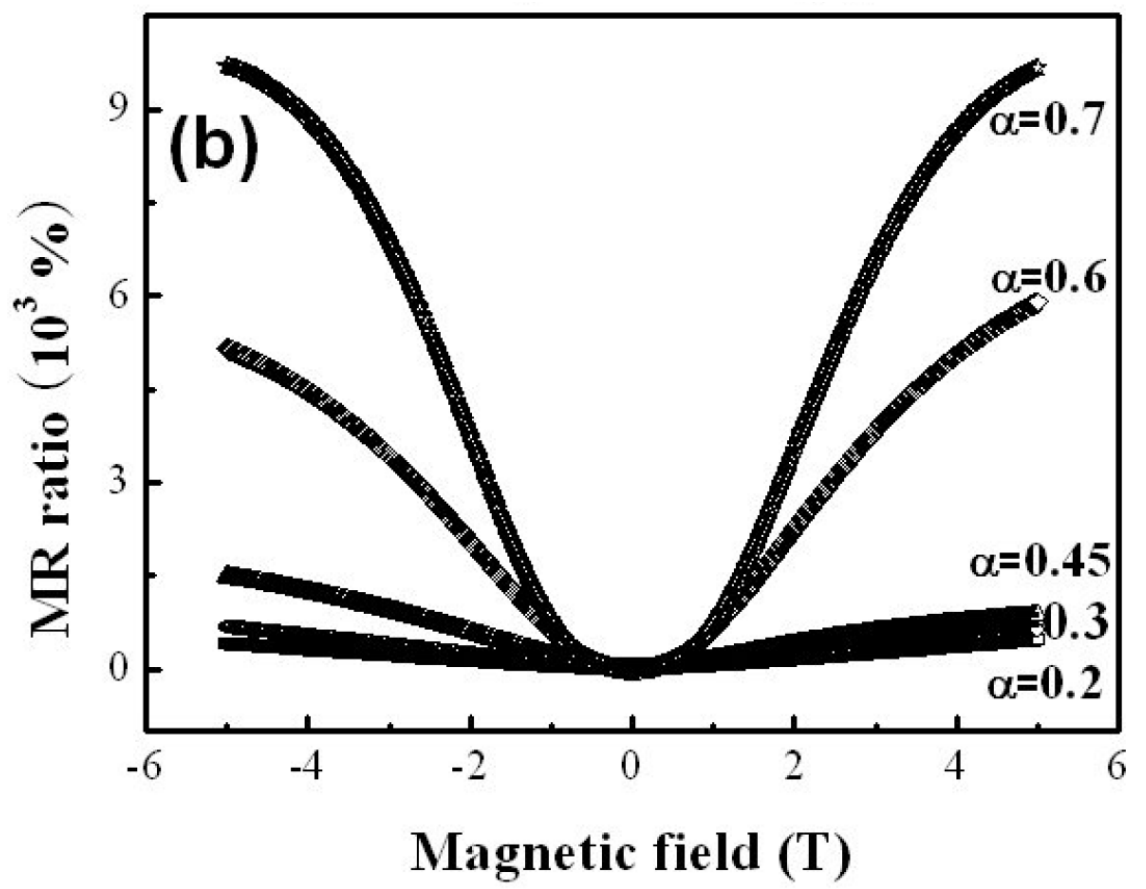

Figure 3.4: Experimental magnetoresistance results from Suh et al., where the magnetoresistance is plotted as a function of applied magnetic field for five values of filling factor $(\alpha=0.2,0.3,0.45,0.6$ and 0.7$)$. (Reproduced from reference 10).

To understand the mechanism for the EMR effect we first need to acknowledge that all magnetoresistance effects are a combination of a physical and a geometrical component 14 . The EMR effect is unusual in that the geometrical component is larger than the physical one. The physical component arises from the orbital motion of the charge carriers caused by the Lorentz force which is reliant on the material parameters. The geometrical contribution is attributed to a field dependent deflection of current around the metallic region, which is in turn dependent on the geometry of the system and the placement of the electrical contacts 10114 .

The EMR effect relies on the switching between a state of low resistance at zero magnetic field to a state of high resistance for large magnetic fields. This redistribution of current in circular geometry systems arises since the electric field lines align perpendicular to the semiconductor-metal interface as the metallic region is an equipotential surface. With no magnetic field the conductivity tensor is diagonal (Equation 2.16) and the current flows along the electric field lines into the metal (short circuit). With the application of a large magnetic field the off-diagonal elements of the conductivity tensor become dominant and the current flows perpendicular to the electric field, i.e. the Hall angle approaches $90^{\circ}$. This forces the current to avoid the conducting region and flow through 
the semiconducting outer disk (open circuit). This transition produces a geometrical magnetoresistance effect even if the physical contribution is zero $\underline{9}$.

The magnetoresistance of such circular modified van der Pauw geometries has also been measured by Suh et al. with the magnetoresistance results presented in Figure 3.4. Here, the magnetoresistance values are smaller in magnitude than those of Solin et al. but still dramatic in comparison to other previously discovered magnetoresistance effects. Room temperature magnetoresistance values of $614 \%$ and $9,900 \%$ were reported for applied magnetic fields of $1 \mathrm{~T}$ and $5 \mathrm{~T}$ respectively in a system with filling factor of $0.7^{10}$. We see from Figure 3.4 that the magnetoresistance values increase rapidly above a filling factor of 0.45 . Below this, the magnetoresistance is not higher than $1,500 \%$ in a $5 \mathrm{~T}$ magnetic field. Saturation of the magnetoresistance is particularly evident at large filling factors for high magnetic fields. The magnetoresistance was found to increase monotonically with filling factor for all values of magnetic field.

The systems measured by Suh et al. are very similar to those of Solin et al. in that they are of the same circular disk geometry and are created from the same materials (InSb and $\mathrm{Au}$ ). However, the specific material parameters (determined by Hall measurements) do vary and are summarised in Table 3.2 . Here, we notice the mobility of indium antimonide is much lower than that in Table 3.1, due to the quality of the thin films in each case. The diameter of the semiconducting disk is $0.1 \mathrm{~mm}$ (the systems are 10 times smaller than those of Solin et al.), with the following values of filling factor investigated: $\alpha=$ $0.2,0.3,0.45,0.6$ and 0.7. The magnetoresistance measurements were carried out with the same van der Pauw contact method as used by Solin et al. in a magnetic field of up to $5 \mathrm{~T}$.

\begin{tabular}{lccc}
\hline & $\begin{array}{c}\text { Conductivity } \\
\sigma(\Omega \mathrm{m})^{-1}\end{array}$ & $\begin{array}{c}\text { Mobility } \\
\mu\left(\mathrm{cm}^{2} / \mathrm{Vs}\right)\end{array}$ & $\begin{array}{c}\text { Carrier Conc. } \\
\mathrm{n}\left(\mathrm{m}^{-3}\right)\end{array}$ \\
\hline $\mathrm{InSb}$ & 81.3 & 12,230 & $4.15 \times 10^{20}$ \\
$\mathrm{Au}$ & $4.10 \times 10^{7}$ & 50 & $5.13 \times 10^{28}$ \\
\hline
\end{tabular}

TABLE 3.2: Material parameters of the EMR systems of Suh et al. at zero magnetic field and at room temperature ${ }^{10}$

The samples were grown on an oxidised (100) silicon (Si) substrate. Initially, a 200nm insulating $\mathrm{SiO}_{2}$ layer was deposited via plasma enhanced chemical vapour deposition. The InSb film (thickness of $1 \mu \mathrm{m}$ ) was thermally evaporated on top of the insulating layer with the microstructure of the film examined via X-ray diffraction and scanning electron microscopy. The systems were then patterned into the circular van der Pauw disk geometry using photolithography, with induced coupled plasma reactive ion etching used to create the concentric hole of varying filling factors. The holes were metallised with a $\mathrm{Ti} / \mathrm{Au}$ stack (gold being the dominant component) and the contact pads finally 
deposited via DC magnetron sputtering and fabricated via a lift off process ${ }^{10}$. Figure 3.5 shows a typical system produced in this way.

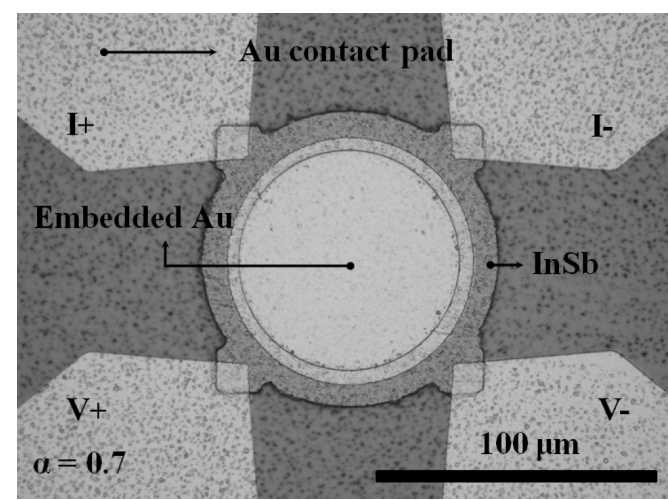

FiguRE 3.5: EMR system geometry used by Suh et al., where the disk diameter is $0.1 \mathrm{~mm}$ and the filling factor is $\alpha=0.7$. The system is comprised of an outer InSb disk with an embedded $\mathrm{Au}$ droplet, with four contacts placed around the perimeter of the disk. (Reproduced from reference 10).

The magnetoresistance of circular geometry EMR devices comprised of InSb and Au have also been reported by Terra et al., however the magnetoresistance values are much smaller than those previously mentioned in this section $\underline{84}$. These systems are significantly large (18mm diameter) and the material parameters for the systems are not given. The systems construction is somewhat unclear, thus making it hard to reproduce the results using FEM analysis. The small magnetoresistance values in these systems may be due to low mobility thin film semiconductors or another aspect of the systems construction.

The systems described until now have been composed of semiconducting InSb containing a circular Au droplet. However, systems with the same geometry but with different materials have been produced. Work by Friedman et al. and Lu et al. are examples of two such systems, both using graphene as the semiconducting material ${ }^{11112}$.

Friedman et al. have reported magnetoresistance values of up to $600 \%$ in a magnetic field of $12 \mathrm{~T}$ at $4.2 \mathrm{~K}^{11}$. The system responsible for these results is formed from monolayer graphene films grown by chemical vapour deposition on copper foils. The graphene films were transferred to a thermally grown $\mathrm{SiO}_{2} / \mathrm{Si}$ substrate layer of thickness 300nm. The metallic shunts were fabricated using photolithography with the deposition of $\mathrm{Ti} / \mathrm{Au}$ via electron-beam assisted deposition. Magnetoresistance measurements were carried out at $4.2 \mathrm{~K}$ using a current of $10 \mu \mathrm{A}$. Unshunted devices (purely graphene) were reported to have a mobility of $2,500 \mathrm{~cm}^{2} / \mathrm{Vs}$ with the resulting magnetoresistance values much lower than the shunted devices (magnetoresistance of $20 \%$ with a $12 \mathrm{~T}$ magnetic field at $4.2 \mathrm{~K}$ ). Raman spectroscopy was used in order to verify the presence of monolayer graphene. It was noted that conventional semiconductors such as InSb are not ideal when the device is reduced in size below $100 \mathrm{~nm}$ as the mobility is rapidly reduced at these length scales, 
which is not the case for graphene. Figure 3.6 shows the magnetoresistance as a function of magnetic field reported by Friedman et al. for various values of gate voltage.

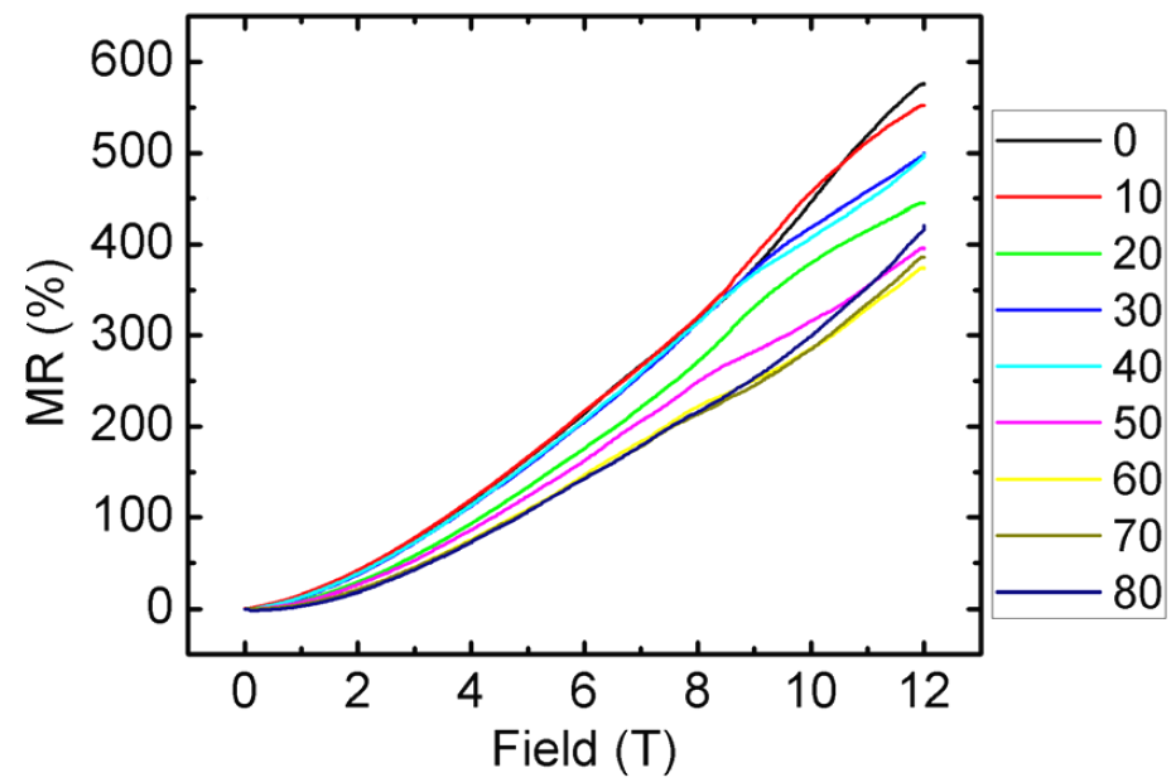

FIGURE 3.6: Magnetoresistance as a function of magnetic field for a circular geometry graphene based EMR device containing a Ti/Au metallic inclusion for various values of gate voltage (shown in the key in units of volts). The magnetoresistance measurements were carried out at $4.2 \mathrm{~K}$ and we see a maximum value of $600 \%$ in a magnetic field of 12T. (Reproduced from reference 11).

Lu et al. have reported a magnetoresistance of up to $55,000 \%$ in a $9 \mathrm{~T}$ applied magnetic field at $300 \mathrm{~K}^{12}$. The system responsible for this large effect, like the one of Friedman et al., uses graphene as the semiconducting material in the same circular disk geometry as seen in Figure 2.10. However, the metallic inclusion is formed from palladium. Figure 3.7 presents a scanning electron microscope image of the system showing the outer graphene ring with four contacts and a central metallic palladium inclusion. With a filling factor of 1 (a purely palladium disk) the magnetoresistance was measured to reach less than $5 \%$ in a $9 \mathrm{~T}$ magnetic field. While for a filling factor of 0 (graphene disk) the magnetoresistance was measured to lie between $300 \%$ and $500 \%$ for various graphene flakes in a magnetic field of 9T.

The graphene films in such systems were produced by mechanical exfoliation from natural graphite with the formation of monolayers, again verified with Raman spectroscopy. The circular van der Pauw geometry was etched into the graphene by oxygen plasma. The electrodes and the concentric metallic disk were created by electron-beam lithography with a $60 \mathrm{~nm}$ metallic film of palladium formed by electron beam evaporation. The mobility of the graphene used in these systems was measured as between 0.4 and $0.7 \mathrm{~m}^{2} / \mathrm{Vs}$. The exact material parameters for this system are not explicitly stated but can be estimated and are summarised in Table 3.3 (the values given are for a gate voltage of $-8 \mathrm{~V}$ ). 


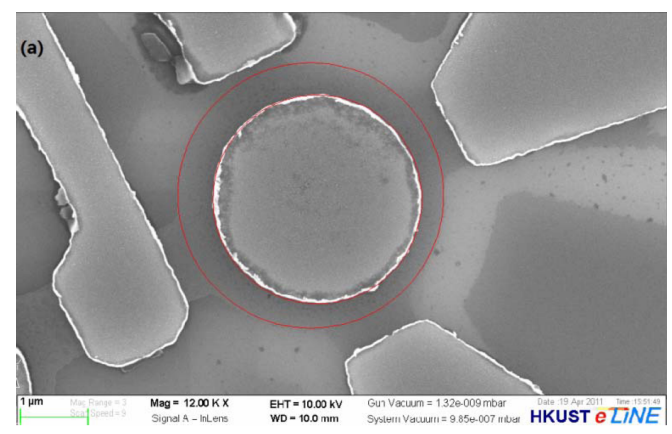

FIGURE 3.7: Scanning electron microscope image of the experimental EMR systems of $\mathrm{Lu}$ et al. indicating the four contacts placed around the perimeter of a graphene disk with a central embedded palladium conducting inclusion (indicated by red lines). Here, the metallic inclusion is not centred exactly in the graphene disk. (Reproduced from reference (12).

\begin{tabular}{lccc}
\hline & Conductivity & Mobility \\
$\sigma(\Omega \mathrm{m})^{-1}$ & $\mu\left(\mathrm{cm}^{2} / \mathrm{Vs}\right)$ & $\begin{array}{c}\text { Carrier Conc. } \\
\mathrm{n}\left(\mathrm{m}^{-3}\right)\end{array}$ \\
\hline Graphene & $3.8 \times 10^{4}$ & 5,000 & $4.75 \times 10^{23}$ \\
$\mathrm{Pd}$ & $1 \times 10^{7}$ & 9.2 & $6.79 \times 10^{28}$ \\
\hline
\end{tabular}

TABLE 3.3: Material parameters of the EMR systems of Lu et al. at zero magnetic field and at room temperature ${ }^{12}$

Four probe measurements were carried out at $300 \mathrm{~K}$ to eliminate the effect of contact resistance. Figure 3.8 shows the magnetoresistance results for these systems with a filling factor of $3 / 4$ for three values of gate voltage $(-8 \mathrm{~V},-6 \mathrm{~V}$ and $-3 \mathrm{~V})$. The experimental results are represented by the solid lines while the points represent the results from their independent FEM simulations. Here, we see a very large magnetoresistance effect of up to $55,000 \%$ in a $9 \mathrm{~T}$ applied magnetic field at $300 \mathrm{~K}$. The magnetoresistance results are asymmetric with respect to the applied magnetic field. This has been attributed to the alignment error of the central metallic region, which is not perfectly central in the graphene disk. This was due to imperfection in the electron beam lithography and etching and has been verified by their simulations. It is noted that a much higher magnetoresistance could be achieved in such a system with the increase in the mobility of graphene (to approximately $1 \mathrm{~m}^{2} / \mathrm{Vs}$ ). The effect of changing the filling factor was also investigated. This was achieved by producing other systems with gold as the material for the central metallic disk (60nm thickness with a $3 \mathrm{~nm}$ Ti adhesion layer). In general, the largest values of magnetoresistance at a given magnetic field were found in systems with large filling factors. This is not strictly true at low magnetic fields (below 1T) where the magnetoresistance was found to peak for filling factors between 0.7 and 0.8 . 


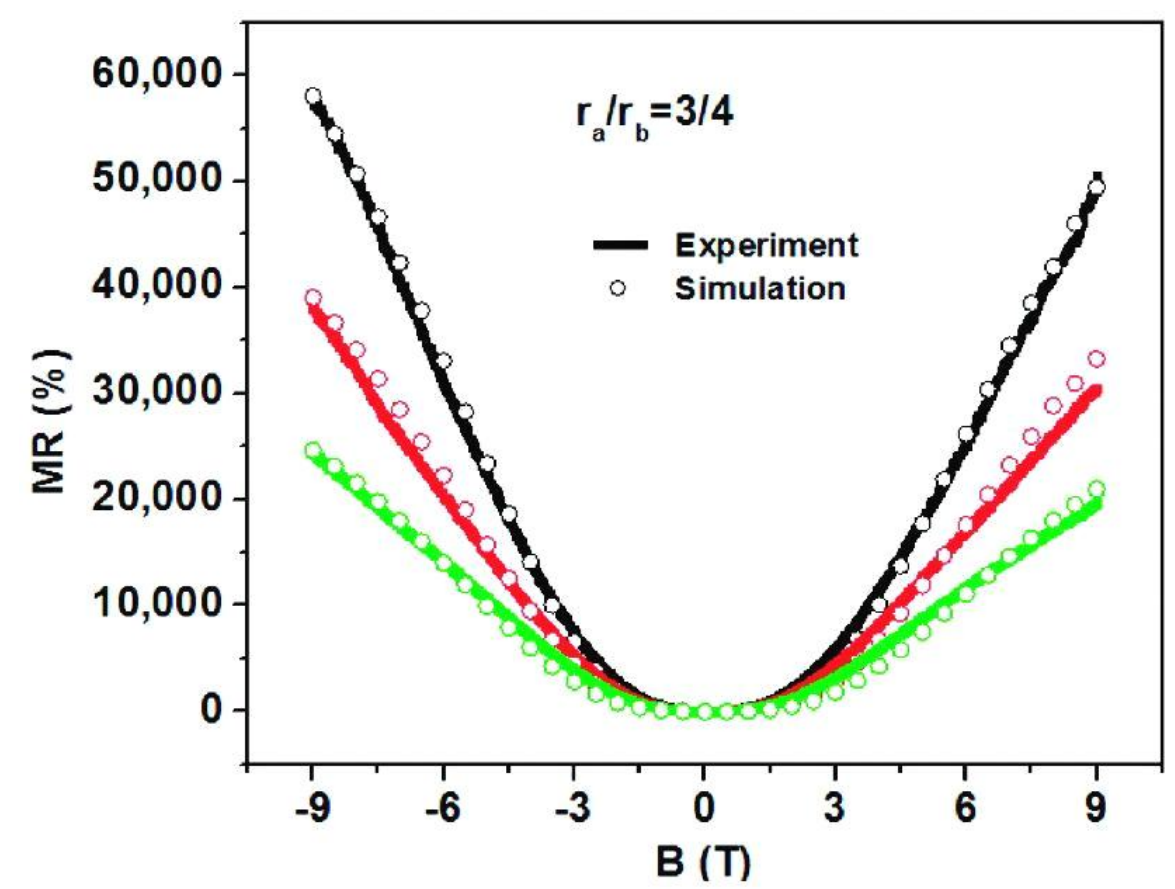

FiguRE 3.8: Magnetoresistance as a function of magnetic field for EMR systems of Lu et al. with the system geometry as given in Figure 3.7 with filling factor of 3/4. Results are given for three values of gate voltage: $-8 \mathrm{~V}$ (black); $-6 \mathrm{~V}$ (red); and $-3 \mathrm{~V}$ (green). Here, the experimental data is represented by the solid lines with the empty circles representing simulations. The asymmetric results are due to the metallic region being misaligned from the centre of the graphene disk. (Reproduced from reference 12).

\subsubsection{FEM Modelling}

The investigation of the EMR effect with the use of the FEM has been considered in literature for both circular and linear geometry devices. Here, the results for circular geometry devices are summarised. In 2001, Moussa et al. reported the modelling of EMR disks with the use of the FEM ${ }^{13}$. Their system was simplified in that a $2 \mathrm{D}$ model was adopted and the contact resistance between the semiconductor and the metal was not included in calculation. The material parameters were the same as those given in experiments and can be seen in Table 3.1 .

The material parameters were used to create the components of the conductivity tensor as given in Equation 2.41. The system contained four contacts placed equidistant around the disks perimeter each with a width of $8^{\circ}$. As in the experimental systems of Solin et al. two adjacent contacts were used for current input and output while the remaining contacts were used to measure the change in potential. The boundary conditions used were electrical insulation on the disk perimeter $(\mathbf{J}=0)$ and continuity at the semiconductor metal interface $\left(\mathbf{n} \cdot \mathbf{J}_{1}=\mathbf{n} \cdot \mathbf{J}_{2}\right)$. The system was described by Equation 2.48 with solutions for the potential produced for the following filling fractions: $16 \alpha=0,6,8,9,10,11,12$, 13, 14 and 15. The mesh used for simulation contained 6,000 nodes with the formation 

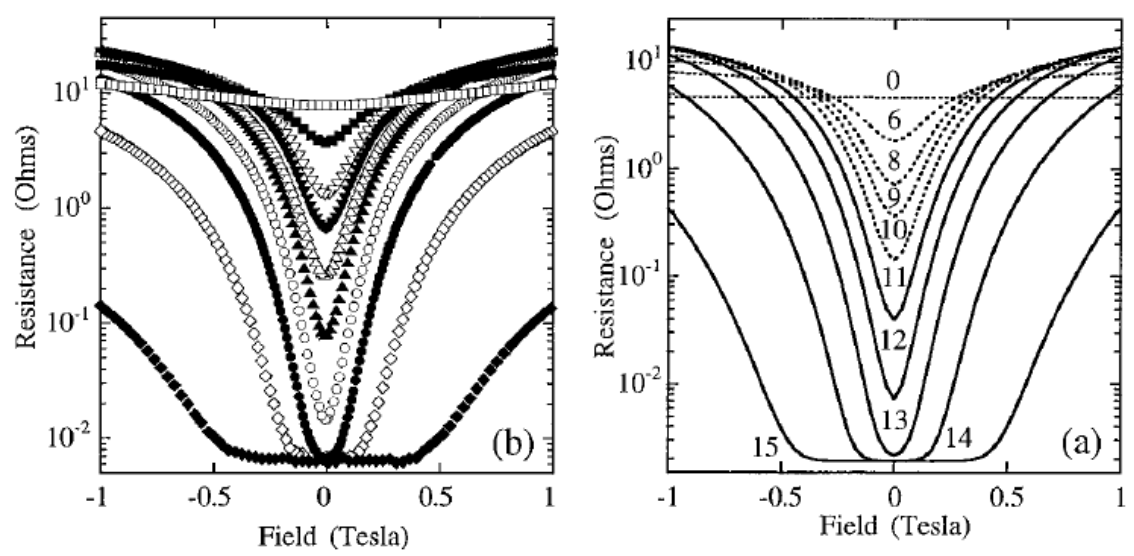

FIGURE 3.9: System resistance as a function of magnetic field for various values of filling factor: (left) the experimental results from Solin et al. where the filling factors were: $16 \alpha=0(\square) ; 6(\bullet) ; 8(\nabla) ; 9(\nabla) ; 10(\triangle) ; 11(\Delta) ; 12(\bigcirc) ; 13(\bullet) ; 14(\diamond) ;$ and 15 $(\bullet)$. (Right) the results obtained from the FEM model (here the numbers represent values of 16 $\alpha$ ). (Adapted from reference 13).

of triangular elements. An excellent agreement between the finite element simulations and experimental results was found (with no adjustable parameters). This can be seen in Figure 3.9 where the systems resistance is given for both the FEM model and an equivalent experimental system. From Figure 3.9 the presence of a magnetoresistance effect is apparent however no magnetoresistance results are explicitly presented in the literature. Figure 3.10 shows the current flow throughout the system for applied magnetic fields of $0 \mathrm{~T}$ and $1 \mathrm{~T}$. Here, the current flow in the system for two values of magnetic field (0T and $1 \mathrm{~T})$ are shown.
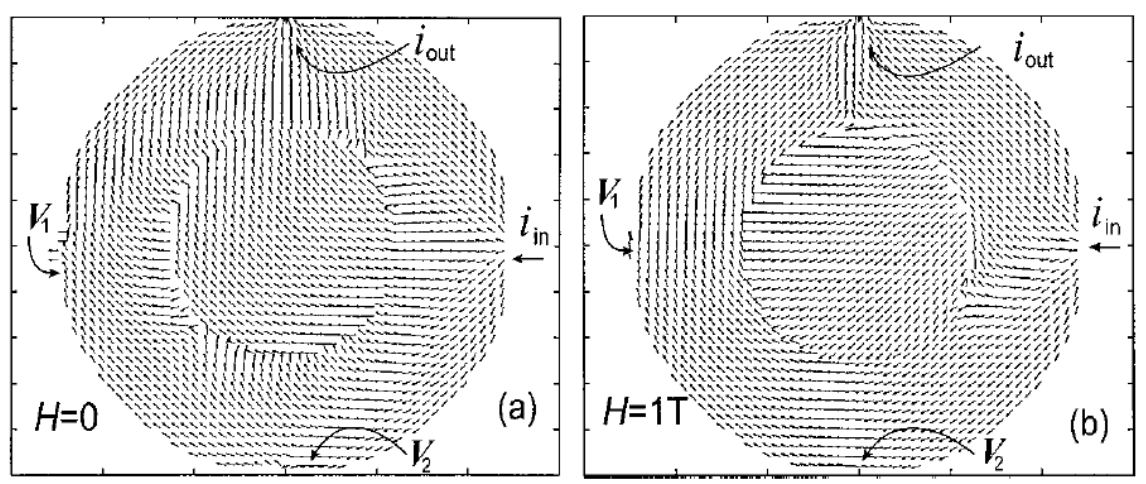

Figure 3.10: The current flow in a modified van der Pauw disk geometry, the outer disk consisting of InSb with a concentric Au metallic inclusion: (a) zero magnetic field and (b) magnetic field of 1T. The lengths of the arrows are not to scale. (Adapted from reference 13).

The model does not account for the physical component of the magnetoresistance (which should be small) or the contact resistance at the semiconductor-metal interface. Despite this it was concluded that the FEM can accurately reproduce experimental EMR results. It was noted that due to the large change in potential near the current contacts that 
EMR results would be very sensitive to the contacts positions. Also, the use of FEM models is suggested for future exploration of systems with more complex geometries for increased performance ${ }^{13}$.

\subsection{Modelling Procedure}

In order to model circular geometry EMR systems, as discovered by Solin et al., the system geometry was created in Comsol Multiphysics. The geometry mimicked that of the experimental system (see Figure 3.1) with some simplifications. These simplifications meant that only the active layers of the device were considered in a $2 \mathrm{D}$ model. The additional thin film layers required experimentally were excluded. The experimental systems can be considered in two dimensions since the thickness of the active layer is much smaller than the diameter of the system and the Lorentz force acts in the x-y plane.

The contacts were modelled as point contacts placed equidistant around the disks perimeter. This means that the effective size of the contacts is equal to the size of the mesh elements connected to a single node in the mesh. In this Thesis the effective size of the contacts varies between approximately $0.1 \%$ and $0.5 \%$ of the systems perimeter

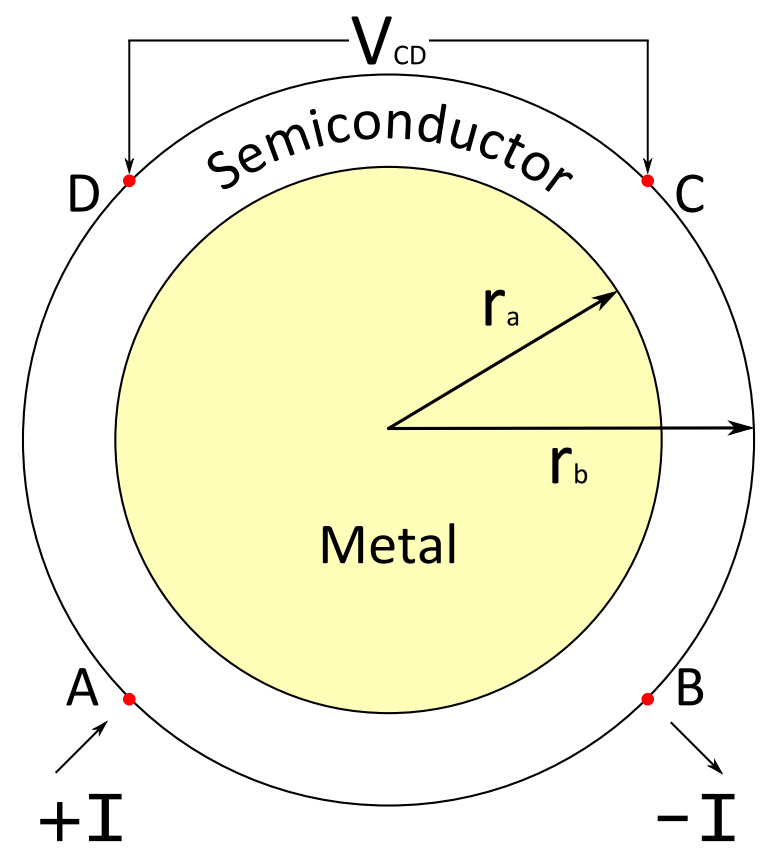

FIGURE 3.11: Simulated EMR disk geometry based on the systems of Solin et al. showing the four point contacts placed equidistant around the perimeter of a semiconducting disk (of radius $r_{b}$ ). The metallic droplet (of radius $r_{a}$ ) is embedded into the centre of the semiconductor. The current is input into the system through contact A and output at contact $\mathrm{B}$. The resulting potential difference across contacts $\mathrm{C}$ and $\mathrm{D}$ is then measured.

This system shows a filling factor of $12 / 16$. 
per contact depending on the specific mesh size used in each model. An image of the simulated system is presented in Figure 3.11. The radius of the entire system $\left(r_{b}\right)$ was set at $0.5 \mathrm{~mm}$ with the radius of the metallic disk $\left(r_{a}\right)$ varied in order to produce filling factors ranging from $1 / 16$ to $15 / 16$ in increments of $1 / 16$. This is in accordance with the specification used by Solin et al. experimentally.

In order to define the conductivity tensor for the semiconducting and metallic regions the material parameters (zero field conductivity and charge carrier mobility) were required. This results in a conductivity tensor that is dependent on system coordinate $(\sigma(\mathrm{x}, \mathrm{y}))$. The material parameter values for each of the experimental systems investigated herein are given in Tables 3.1, 3.2 and 3.3. The material parameters represent values measured at room temperature, therefore the models produced using these values refer to room temperature results.

All models in this chapter have the same boundary conditions. At the system boundary (outer edge of the semiconducting disk) the normal component of the current density was set to zero $(\mathbf{n} \cdot \mathbf{J}=0)$. This meant that no external current entered the system and is referred to as electric insulation in Comsol Multiphysics. At the semiconductormetal interface the normal component of the current density was made continuous $\left(\mathbf{n} \cdot\left(\mathbf{J}_{1}-\mathbf{J}_{2}\right)=0\right)$, referred to as continuity in Comsol Multiphysics.

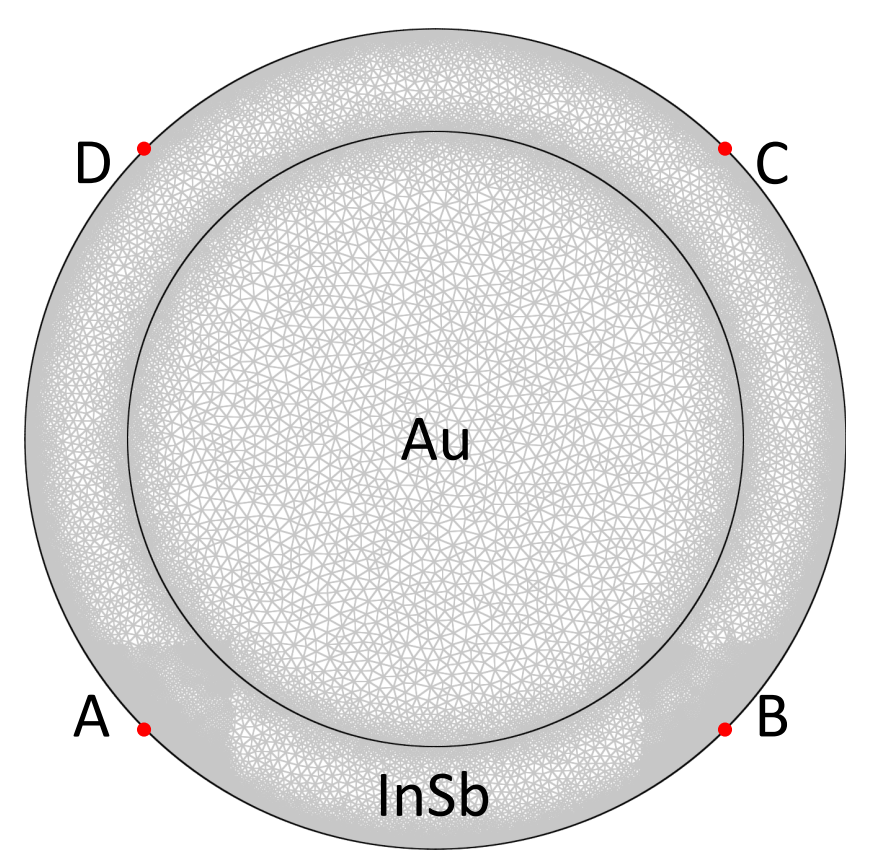

Figure 3.12: A typical mesh used for simulations for a system with a filling factor of $12 / 16$. Here, the mesh is more refined around the system perimeter and at the semiconductor-metal interface. The mesh consists of approximately 60,000 triangular mesh elements and has been refined around the current contacts ( $\mathrm{A}$ and $\mathrm{B}$ ) where the variation in potential is greatest. 
For the measurement of the EMR effect a constant current was input at contact A and output at contact $\mathrm{B}$. The resultant potential difference between the remaining two contacts $(\mathrm{C}$ and $\mathrm{D})$ was measured (as seen in Figure 3.11). Since the magnetoresistance does not depend on the magnitude of the current, the value was set at $1 \mu \mathrm{A}$ in order to achieve realistic voltages.

The system geometry was discretised by producing a triangular mesh. Since the electric potential varied by a larger amount around the outer edge of the disk (especially near the current contacts) and at the semiconductor-metal interface, the mesh was more refined at these locations than the rest of the system. The metallic inhomogeneity is effectively an equipotential surface, therefore the mesh inside the metallic region was not as refined as inside the semiconducting region. To improve the accuracy of the results, two square regions around current contacts $\mathrm{A}$ and $\mathrm{B}$ were refined. The method of selecting the appropriate mesh is discussed in Chapter 2 . Figure 3.12 shows the general appearance of a typical mesh used for simulations, this mesh contains approximately 60,000 triangular mesh elements.

Before the system was solved it was necessary to define a variable in order to calculate the magnetoresistance. This was achieved using the following expression:

$$
E M R=\frac{R_{A B, C D}(\mathbf{B})-R_{A B, C D}(\mathbf{0})}{R_{A B, C D}(\mathbf{0})}=\frac{V_{C D}(B)}{V_{C D}(0)}-1
$$

Here, $V_{C D}$ is the potential difference between contacts $\mathrm{C}$ and $\mathrm{D}$ and $R_{A B, C D}(\mathbf{B})=$ $V_{C D}(B) / I_{A B}$. The system was then solved for a range of magnetic fields (0 to $5 \mathrm{~T}$ ) using a parametric solver. The postprocessing in Comsol Multiphysics allows for many parameters to be investigated, including: electric potential; current density; and electric field. An example of the code (m-file) used in order to create these models in Version 3.4 of Comsol Multiphysics is given in Appendix D.

\subsection{Modelling in a Circular System Geometry}

In this section the results of the FEM models for circular geometry EMR devices are presented. Firstly, the systems of Solin et al. are verified. With such verification it is shown that the model is applicable to other systems. Such as those reported by Suh et al. and Lu et al., where different material parameters are used.

Figures 3.13 and 3.14 show the magnetoresistance against applied magnetic field for such circular geometry systems at various values of filling factor. The material parameters used were the same as those in the experimental systems of Solin et al., and can be seen 


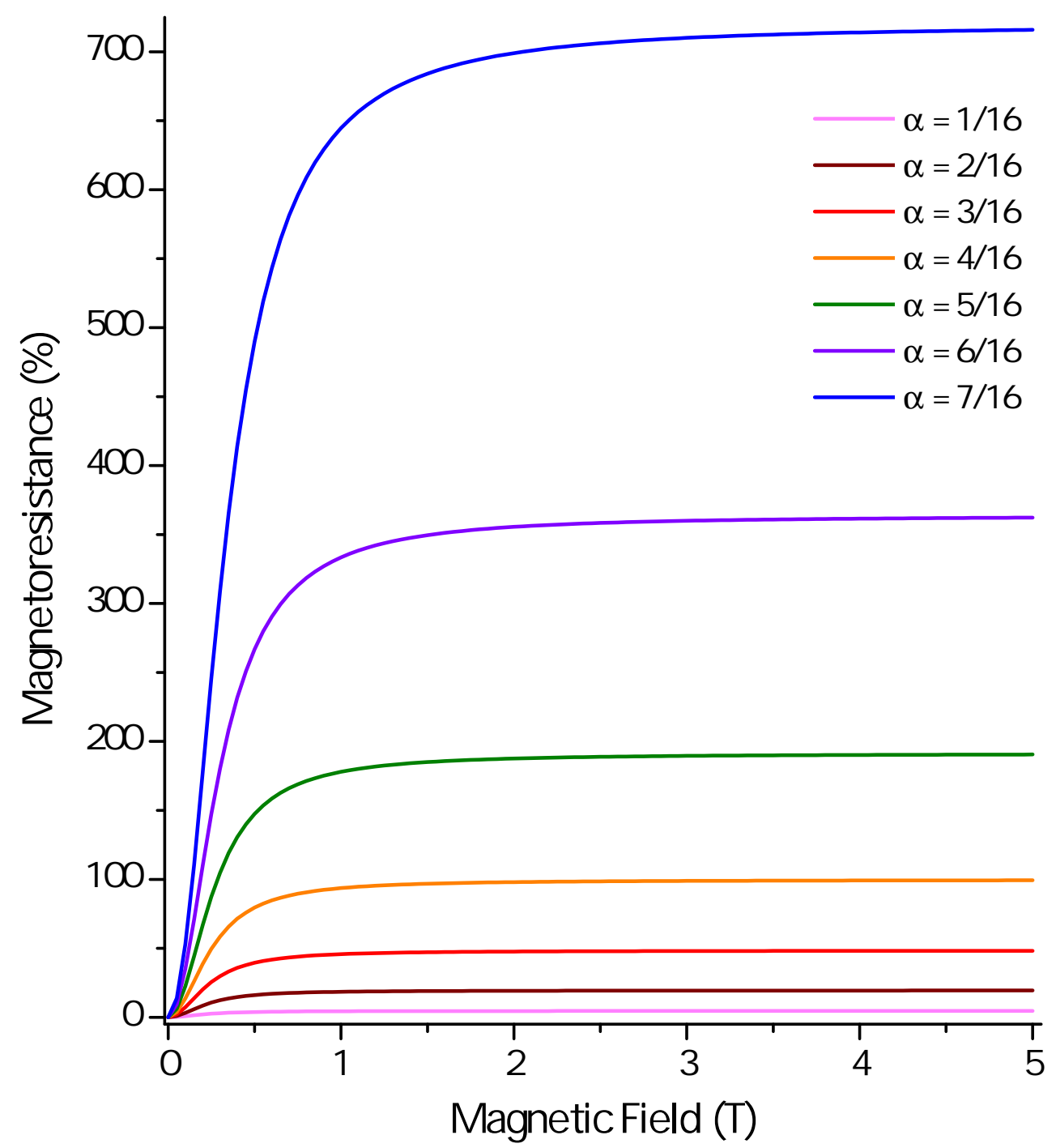

FIGURE 3.13: Magnetoresistance as a function of magnetic field for simulations based on circular EMR systems, for filling factors from 1/16 to $7 / 16$.

in Table 3.1. Here, we see that in general, larger magnetoresistance values occur for greater filling factors with saturation occurring for filling factors from 1/16 to 12/16. The current flow at high magnetic field (directed through the semiconductor) results in a higher system resistance when the filling factor is large as the width of semiconducting material is narrower. The system resistance at low magnetic fields is also reduced at large filling factors since the proportion of the current path that flows inside the metallic region increases, thus resulting in an increased magnetoresistance effect. Filling factors of above 13/16 do not saturate within the $5 \mathrm{~T}$ magnetic field range. The striking result from these figures is the magnitude of the EMR effect with the magnetoresistance reaching $1,730,000 \%$ at a $5 \mathrm{~T}$ magnetic field for filling factors of $13 / 16$. Generally, we see a good agreement with the experimental values of Solin et al. as presented in Figure 3.2 


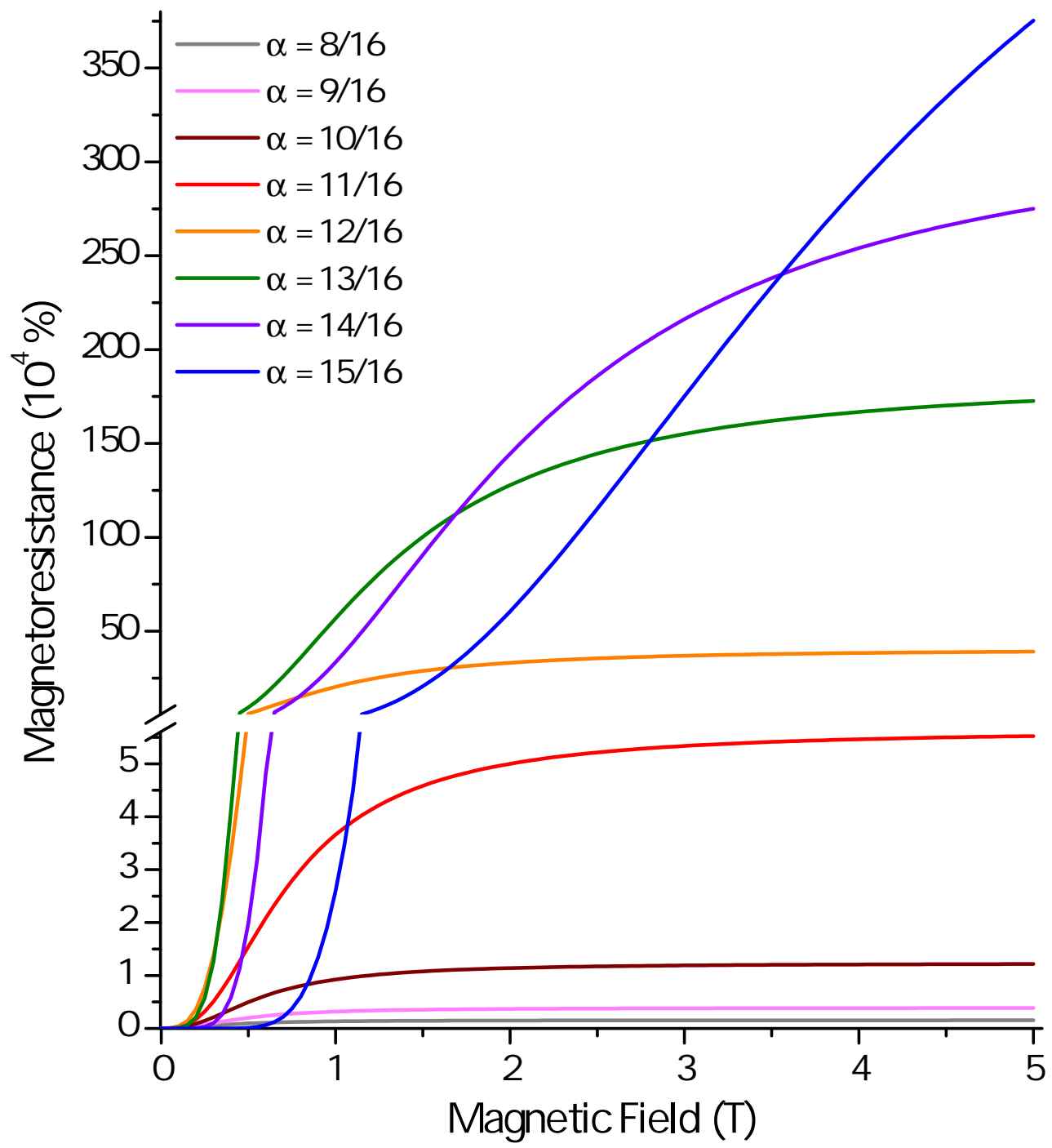

FIGURE 3.14: Magnetoresistance as a function of magnetic field for simulations based on circular EMR systems, for filling factors from 8/16 to 15/16.

Figure 3.15 makes comparison to experimental results more transparent where the magnetoresistance is plotted as a function of filling factors for five values of magnetic field $(0.05 \mathrm{~T}, 0.1 \mathrm{~T}, 0.25 \mathrm{~T}, 1 \mathrm{~T}$ and $5 \mathrm{~T})$. Here, we see that in general the largest values of magnetoresistance occur in systems with a large filling factor and at high magnetic fields. At small magnetic fields $(0.05 \mathrm{~T}, 0.1 \mathrm{~T}$ and $0.25 \mathrm{~T})$ the magnetoresistance values rise monotonically with filling factors from $1 / 16$ up to $12 / 16$. Above this value the magnetoresistance reduces dramatically. With a $1 \mathrm{~T}$ magnetic field the magnetoresistance peaks at a filling factor of 13/16. However, with a magnetic field of $5 \mathrm{~T}$ the magnetoresistance does not peak but continues to increase up to the largest filling factor of 15/16 (the increase in magnetoresistance above a filling factor of 13/16 is much smaller than below this value). In general we see a very good agreement between these results and the experimental results of Solin et al. which are given in Figure 3.3 . However, there 


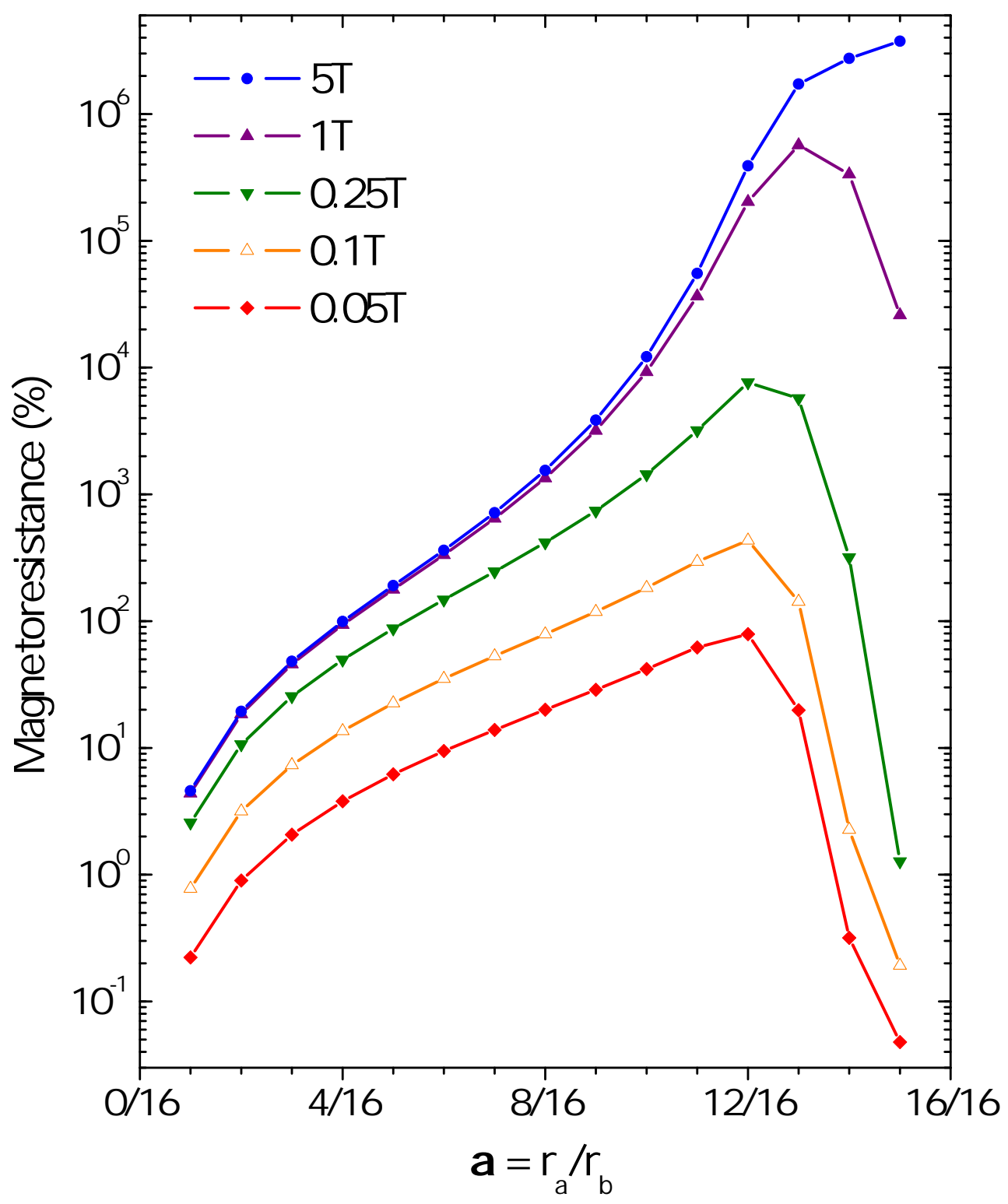

FIGURE 3.15: Magnetoresistance as a function of filling factor for a circular EMR device for five values of magnetic field: 0.05T $(\diamond)$; 0.1T $(\triangle)$; 0.25T $(\nabla)$; $1 \mathrm{~T}(\boldsymbol{\Delta})$; and $5 \mathrm{~T}(\bullet)$. The experimental results of Solin et al. for these systems are presented in Figure 3.3 for comparision.

are some important differences. With a magnetic field of $5 \mathrm{~T}$ the experimental results show a peak in the magnetoresistance at a filling factor of $13 / 16$. The results from the FEM model do not show this behaviour and the magnetoresistance is seen to increase up to a filling factor of $15 / 16$. The model appears to overestimate the magnetoresistance at large filling factors (14/16 and 15/16) and at large magnetic fields (5T). A possible explanation of this result is related to the differences between the idealised FEM model and the real experimental systems. The FEM results are based on a two-dimensional system that has: four point contacts positioned equidistant around the disk perimeter; a precise value of filling factor; and an ideal ohmic contact at the semiconductor-metal 
interface. The model also does not account for the ordinary magnetoresistance of the constituent materials which will always be present when a magnetic field is applied. This physical component of the total magnetoresistance should be small when compared to the extremely large geometrical component especially at high magnetic fields and large filling factors.

The interface between the semiconductor and the metal in the experimental systems was noted as being $19^{\circ}$ away from vertical, meaning the filling factor varied throughout the films thickness. This results in a very small uncertainty in the value of filling factor in the experimental system, but more importantly means that the current flow in the plane of the device does not meet the semiconductor-metal interface at right angles. This allows for the possibility of current entering the metallic region in the direction parallel ( $\mathrm{z}$ direction) to the applied magnetic field. This causes a reduction in the magnetoresistance since current flow in the $\mathrm{z}$ direction is not influenced by the Lorentz force and is not expelled from the metal by the application of magnetic field. Additionally, the contacts used experimentally had a finite size on the disk perimeter and encroached slightly into the semiconducting material. The combination of the finite sized contacts and non vertical semiconductor-metal interface in the experimental system is where the discrepancy at large filling factors and high magnetic field is thought to lie between the model and experimental results. The discrepancy between the experimental and modelled magnetoresistance, caused by finite sized experimental contacts, is increased for larger values of filling factor and for high magnetic fields. At these parameters the majority of the current is forced to flow through the narrow semiconducting region; any encroachment of the contacts would lead to a reduction in the experimental magnetoresistance value.

The model studied in this Thesis is very idealised but is still adequate to show a very good agreement with experimental results. A more sophisticated model specifically tailored to a single experimental system would be expected to produce results closer to those found in experiments, especially at high filling factors and high magnetic fields. In Chapters 4 and 5. modifications to this idealised model are considered and show that an even better agreement to experimental data can be obtained when experimental system details are considered. At low magnetic fields the experimental results are slightly larger than the modelled values. This could be due to the physical contribution to the magnetoresistance that is not considered in FEM simulations, as it is generally much smaller than the geometrical contribution at intermediate and high filling factors. Also, the differences between the modelled and experimental systems are not as significant at small filling factors, since only a small proportion of the current flows through the metallic region at zero field. If the distance between a current contact and the metallic region is greater than half of the displacement between the two current contacts, the lowest resistance route for the current (at zero field) is to avoid the metallic region and flow directly 


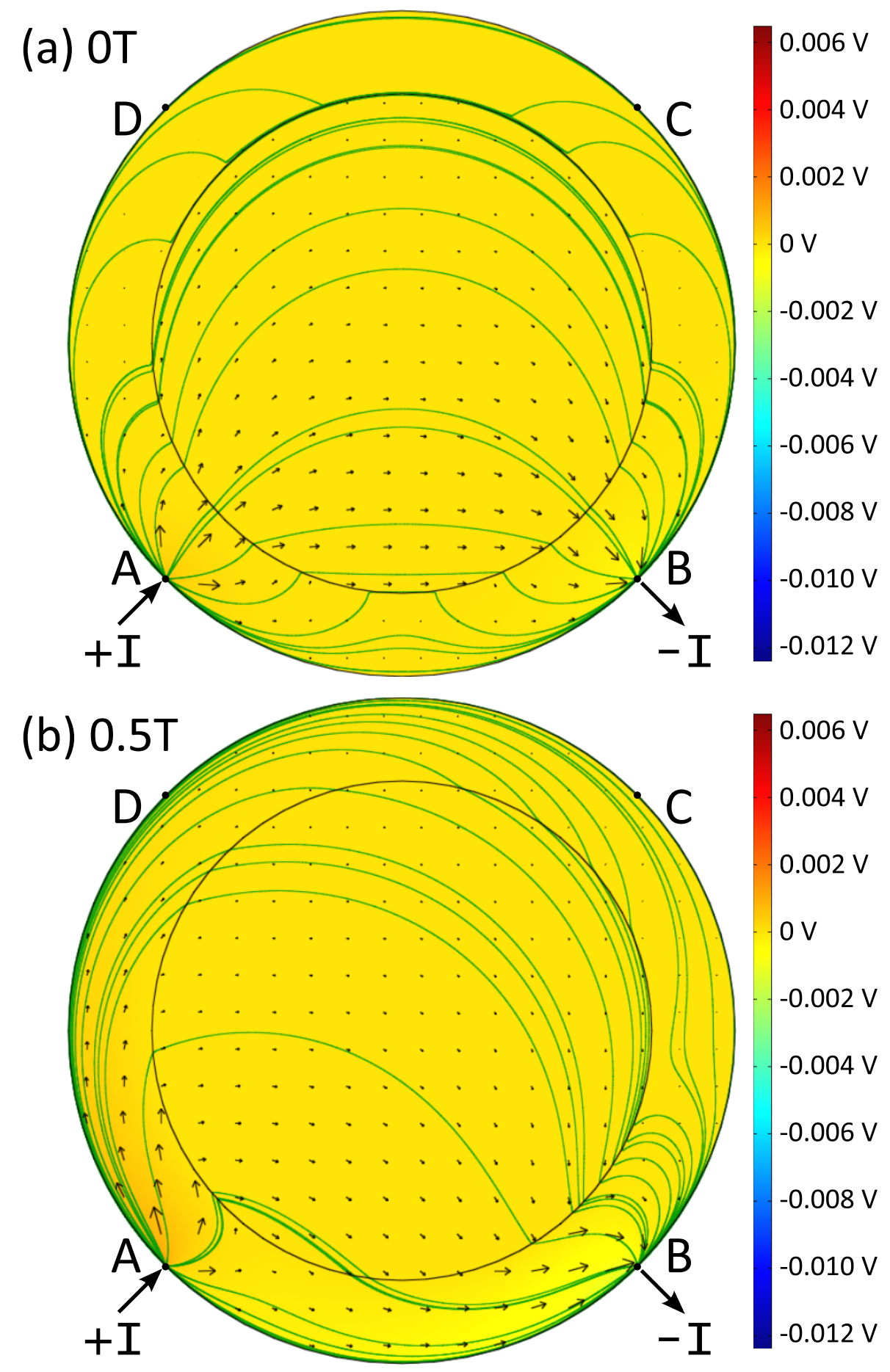

FIGURE 3.16: Visualisation of the current flow and the distribution of electrical potential throughout a modified van der Pauw disk with circular conducting region $(\alpha=12 / 16)$, produced via modelling in Comsol Multiphysics. Here, the background colour represents the distribution of electrical potential with the coloured bar showing the corresponding voltages (in units of volts). The current density is represented by both the green streamlines and the black arrows. The streamlines connect regions with the same current density, while the arrows show the magnitude and direction of the current flow at various points throughout the system. The six images in this figure represent different applied magnetic fields: (a) 0T; (b) 0.5T; (c) 1T; (d) 2.5T; (e) 4T; and (f) $5 \mathrm{~T}$. 


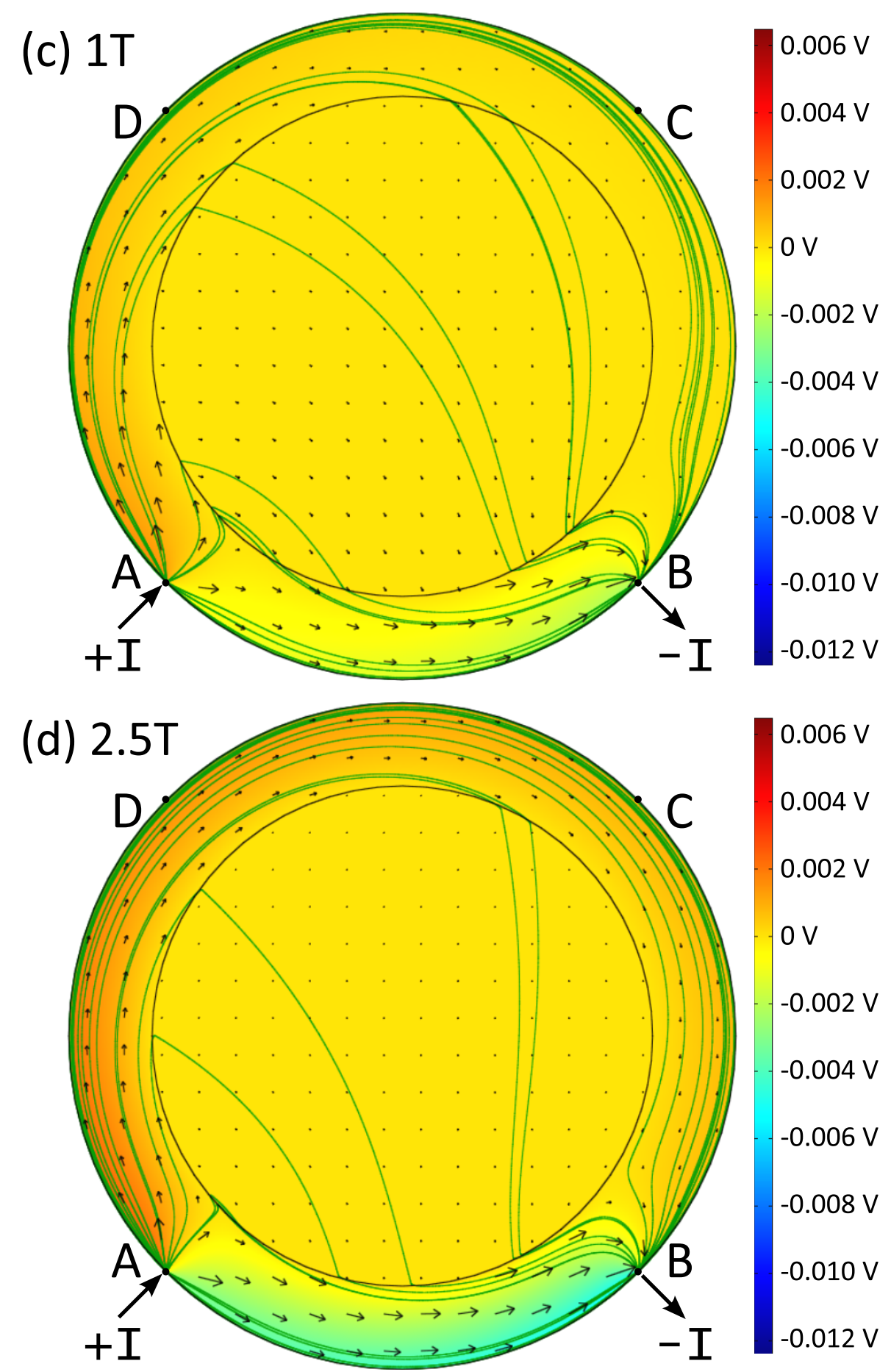

FIGURE 3.16: (Cont.) Visualisation of the current flow and the distribution of electrical potential throughout a modified van der Pauw disk with circular conducting region $(\alpha$ $=12 / 16$ ), produced via modelling in Comsol Multiphysics. Here, the background colour represents the distribution of electrical potential with the coloured bar showing the corresponding voltages (in units of volts). The current density is represented by both the green streamlines and the black arrows. The streamlines connect regions with the same current density, while the arrows show the magnitude and direction of the current flow at various points throughout the system. The six images in this figure represent different applied magnetic fields: (a) 0T; (b) $0.5 \mathrm{~T}$; (c) $1 \mathrm{~T}$; (d) $2.5 \mathrm{~T}$; (e) $4 \mathrm{~T}$; and (f) $5 \mathrm{~T}$. 


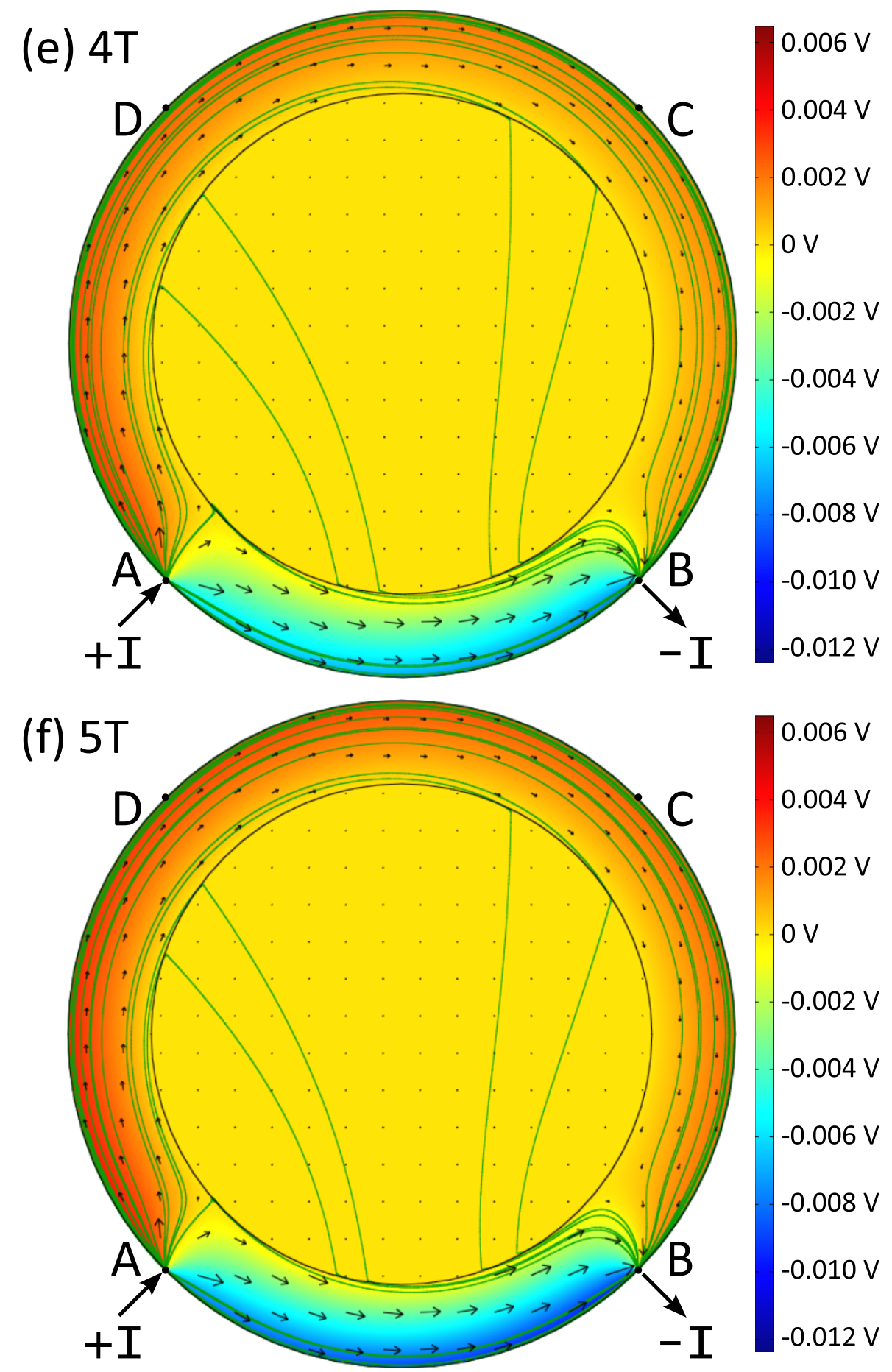

Figure 3.16: (Cont.) Visualisation of the current flow and the distribution of electrical potential throughout a modified van der Pauw disk with circular conducting region $(\alpha$ $=12 / 16)$, produced via modelling in Comsol Multiphysics. Here, the background colour represents the distribution of electrical potential with the coloured bar showing the corresponding voltages (in units of volts). The current density is represented by both the green streamlines and the black arrows. The streamlines connect regions with the same current density, while the arrows show the magnitude and direction of the current flow at various points throughout the system. The six images in this figure represent different applied magnetic fields: (a) 0T; (b) 0.5T; (c) 1T; (d) 2.5T; (e) 4T; and (f) $5 \mathrm{~T}$. 
between the current contacts. Therefore, in systems with small filling factors the zero field resistance of the system will not be substantially less than at high field, resulting in much smaller magnetoresistance values.

The FEM model presented here has been extremely useful with regards to visualising the variation of specific parameters throughout the system for a range of magnetic fields. Figure 3.16 is one such example where the variation in electric potential and current flow in the system are presented for six values of magnetic field in the range of $0 \mathrm{~T}$ to $5 \mathrm{~T}$. The geometry of the system used in this figure has a filling factor of 12/16 and uses the same material parameters as those in Figure 3.15 (see Table 3.1). Figure 3.16 allows for the visualisation of how the EMR mechanism arises. The surface plot shows the variation of the electric potential throughout the system with the scale given by the coloured bar. The green streamlines and the black arrows represent the flow of current. The streamlines connect regions of the same current density while the arrows give an indication of the magnitude and direction of the current flow at various points throughout the system.

Without magnetic field (see Figure 3.16(a)) we see the vast majority of the current flow throughout the system is directed through the conducting disk with almost no current flowing through the semiconducting region around the metal. This constitutes a regime of low overall system resistance, since the conductivity of the metallic region in this model is 2,430 times that of the semiconductor. This behaviour changes upon the application of a magnetic field. In Figure 3.16(b) we see that the current flow has changed. Although some current still flows through the metallic region, the magnetic field has acted to force a proportion of the current to flow through the semiconducting region. This increases the resistance of the system overall. An increase in magnetic field (Figure 3.16(c) - (f)) causes a greater proportion of the current to switch from flowing through the metallic region to flow through the semiconducting region. With the largest magnetic field of 5T in Figure 3.16(f) we see that virtually all of the current is directed through the semiconducting material and very little current flows through the metal. This situation is achieved for this system at a lower field than 5T. Therefore, the current flow at $2.5 \mathrm{~T}$ is very similar to that at $4 \mathrm{~T}$ and $5 \mathrm{~T}$, thus the magnetoresistance saturates (at approximately $1.5 \mathrm{~T}$ for this system).

In order to quantify the current expulsion from the metallic disk at high field a current cross-section has been produced and is given in Figure 3.17. Here, a cross-section of the system has been taken along the vertical diameter of the system. This figure shows where the largest proportion of the current flows from the left-hand side of the system to the right-hand side, for given values of magnetic field. The current density (normalised by the maximum value at the given magnetic field) is plotted, with the numbers in the diagram relating to the position along the cross-section on the plot. 


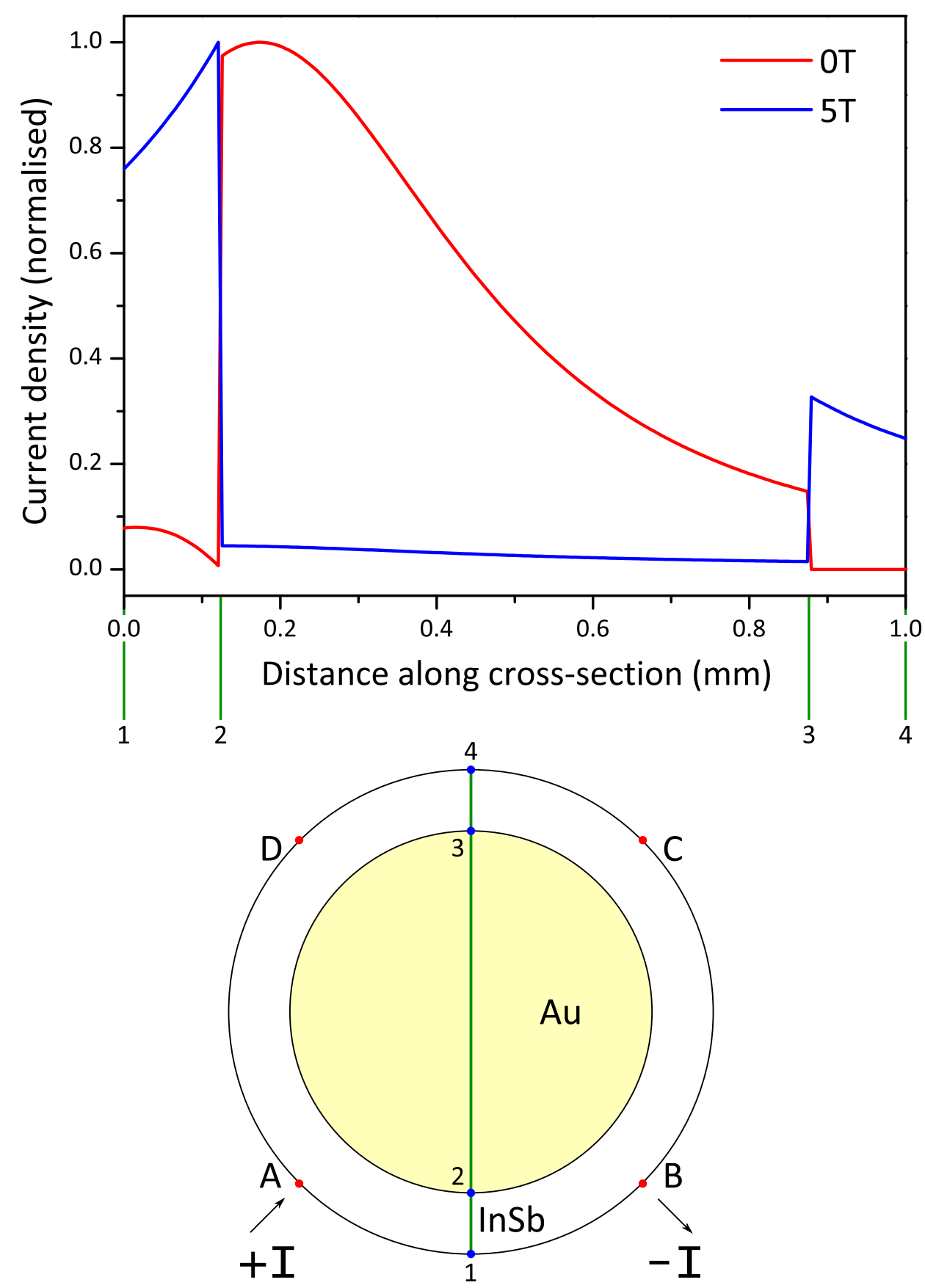

Figure 3.17: Current cross-section of a circular EMR system with a magnetic field of $0 \mathrm{~T}$ and $5 \mathrm{~T}$. Here, the diameter shown in green on the diagram indicates the cross-section that was taken. This line corresponds to the $\mathrm{x}$ axis of the plot with specific points of interest highlighted by the numbers 1 to 4 . The current density (normalised by the largest value in each case) is plotted along this cross-section for magnetic fields of 0T and $5 \mathrm{~T}$. 

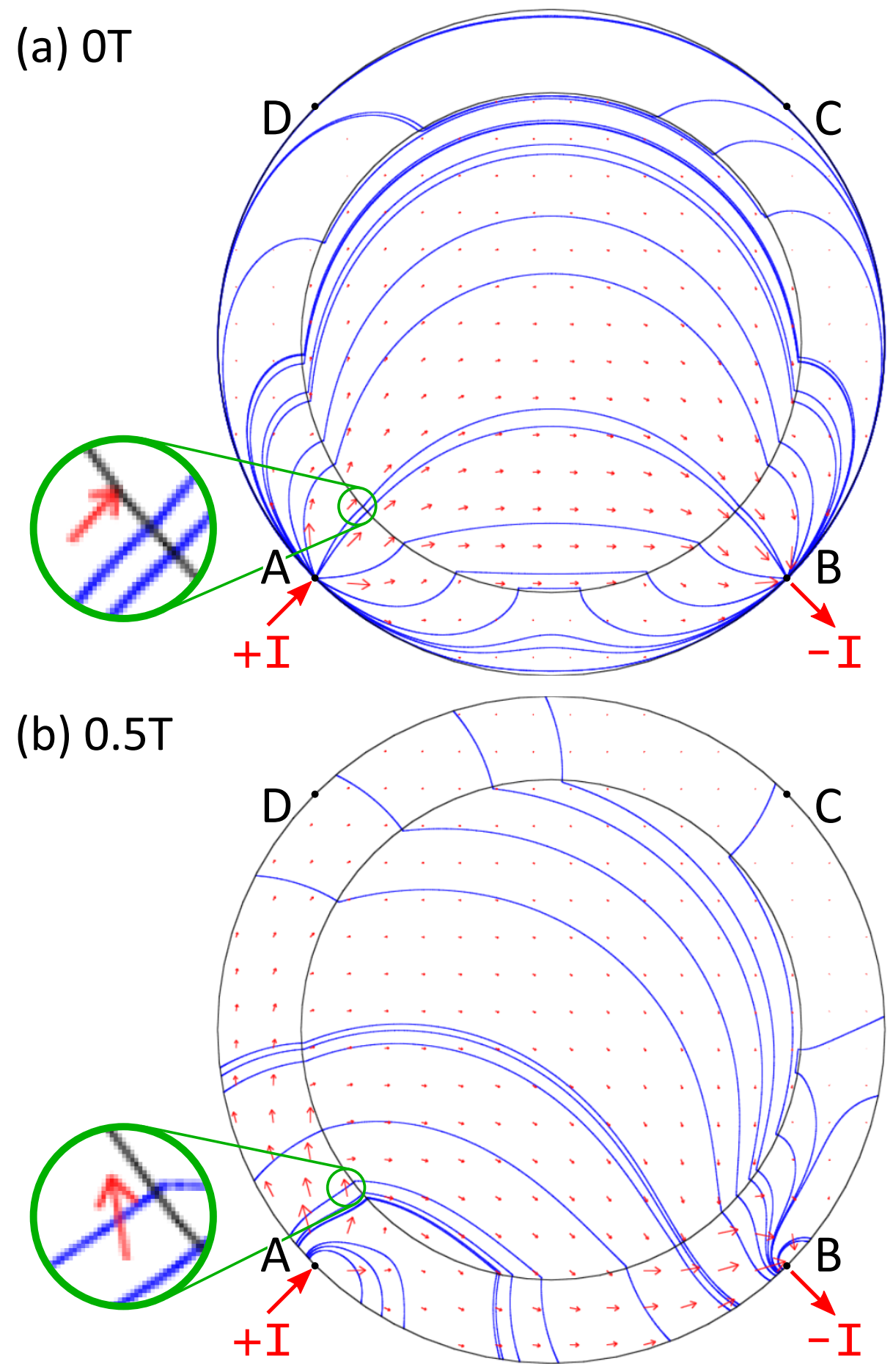

Figure 3.18: Visualisation of the current flow and the electric field lines throughout a modified van der Pauw disk with circular conducting region $(\alpha=12 / 16)$, produced via modelling in Comsol Multiphysics. Here, the electric field lines are shown in blue, while the red arrows represent the magnitude and direction of the current flow at various points throughout the system. The three images in this figure represent different applied magnetic fields: (a) 0T; (b) 0.5T; and (c) 5T. This figure shows how the Hall angle arises in such a system. At zero magnetic field the current and the electric field are aligned parallel (zero Hall angle). At an intermediate field of $0.5 \mathrm{~T}$ we see a non-zero Hall angle, here measured as $66^{\circ}$. Finally, with a large magnetic field of $5 \mathrm{~T}$ we see the current and the electric field are aligned almost perpendicular (Hall angle $\approx 90^{\circ}$ ). 


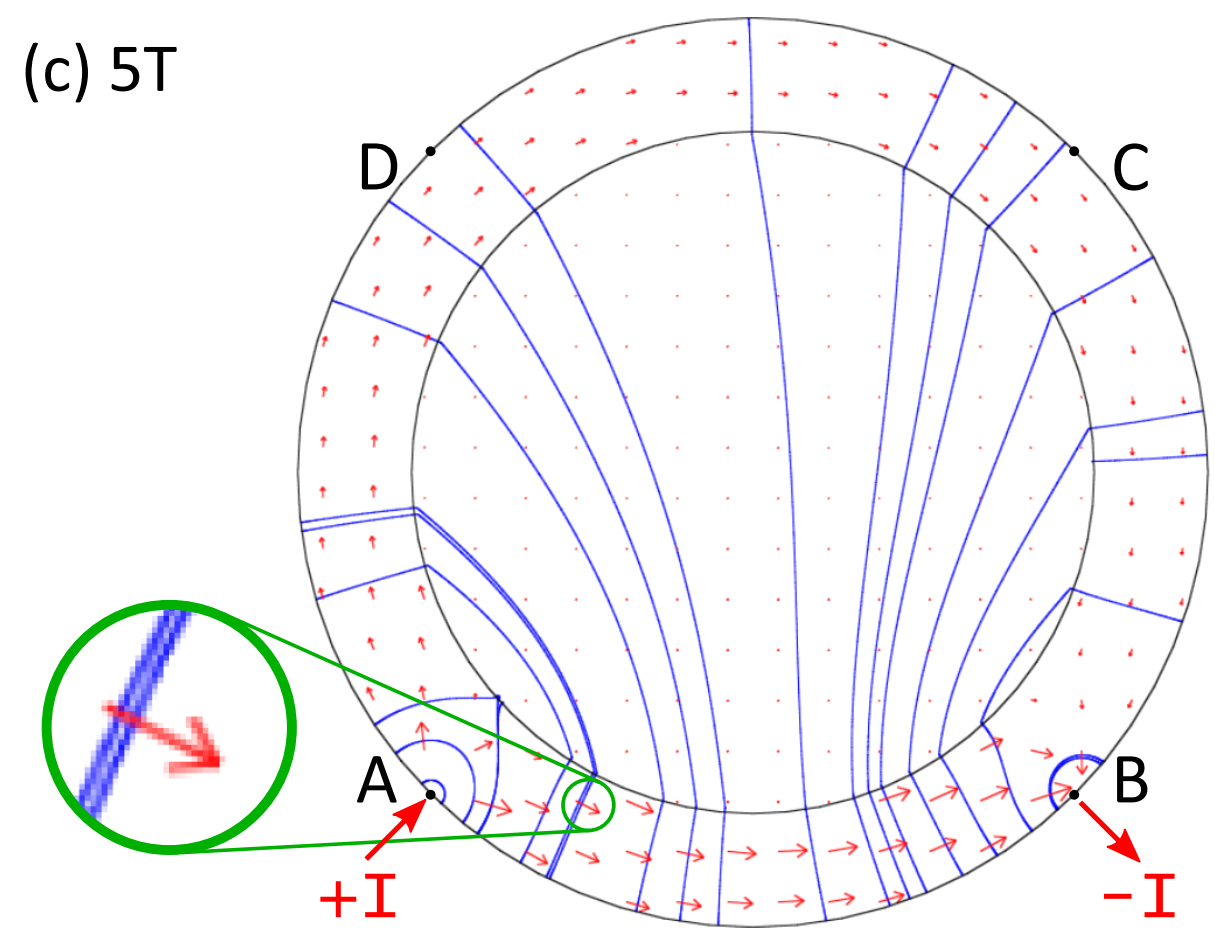

FiguRE 3.18: (Cont.) Visualisation of the current flow and the electric field lines throughout a modified van der Pauw disk with circular conducting region $(\alpha=12 / 16)$, produced via modelling in Comsol Multiphysics. Here, the electric field lines are shown in blue, while the red arrows represent the magnitude and direction of the current flow at various points throughout the system. The three images in this figure represent different applied magnetic fields: (a) 0T; (b) 0.5T; and (c) 5T. This figure shows how the Hall angle arises in such a system. At zero magnetic field the current and the electric field are aligned parallel (zero Hall angle). At an intermediate field of $0.5 \mathrm{~T}$ we see a non-zero Hall angle, here measured as $66^{\circ}$. Finally, with a large magnetic field of $5 \mathrm{~T}$ we see the current and the electric field are aligned almost perpendicular $\left(\right.$ Hall angle $\approx 90^{\circ}$ ).

Figure 3.17 visually shows how the EMR effect arises and quantifies the current switching between $0 \mathrm{~T}$ and $5 \mathrm{~T}$. We see that at zero field the semiconducting regions (between points 1 to 2 and 3 to 4 ) show the lowest values of current flow. The largest current flow occurs in the metallic region (between points 2 and 3). With a magnetic field of $5 \mathrm{~T}$ the current density is shown to have dramatically reduced in the metallic region, where a very small proportion of the current is still flowing. In contrast, the semiconducting regions now have the majority of the current flow travelling through them. This figure reinforces the points made in Figure 3.16 regarding the EMR mechanism, showing the dramatic current switching with the application of a magnetic field.

In order to explain the expulsion of the current from the metallic region at high fields it is necessary to look to Figure 3.18. Here, the current flow is plotted alongside the electric field throughout the system and is used to explicitly show the adaptation of the Hall angle for three values of magnetic field (0T, $0.5 \mathrm{~T}$ and $5 \mathrm{~T})$. Since the metallic region is effectively an equipotential surface we expect that the electric field lines align perpendicular to the 


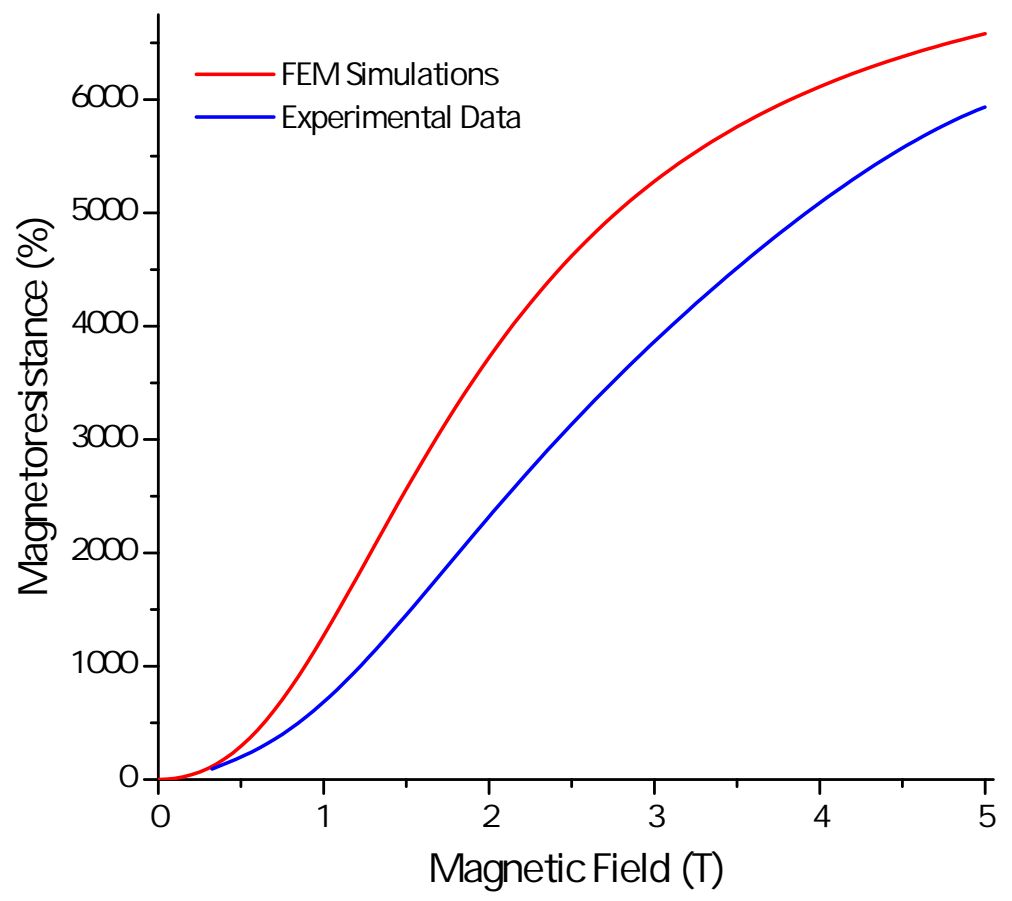

FIGURE 3.19: Magnetoresistance as a function of magnetic field for the FEM model and the corresponding experimental data for the systems of Suh et al. respectively. The system responsible for these results was an order of magnitude smaller than the systems of Solin et al. $\left(r_{b}=0.05 \mathrm{~mm}\right)$ with a filling factor of 0.6. The device was created using semiconducting InSb and metallic Au with the magnetoresistance measured from 0T to $5 \mathrm{~T}$. The material parameters used for simulation were taken from the experimental data and can be seen in Table 3.2

semiconductor-metal interface. At zero magnetic field the current density and the electric field are parallel with one another. This constitutes the low resistance regime, where the current flow is directed into the metallic region. The application of a magnetic field causes a non-zero Hall angle to appear (angle between the electric field and the current density). The application of a sufficiently large magnetic field causes the Hall angle to approach $90^{\circ}$. Since the electric field lines align perpendicular to the semiconductor-metal interface, the lines of current density will align parallel to the interface at large values of magnetic field. This results in the current density avoiding the metallic region and being forced to flow through the semiconducting material, constituting the high resistance regime. The results presented here strongly agree with the EMR mechanism described by Solin et al., which demonstrates that FEM is a very useful tool in the modelling of the EMR effect.

In Figure 3.18 we see that at zero magnetic field the current and electric field lines are parallel at the interface (therefore a Hall angle of zero) as expected. At $0.5 \mathrm{~T}$ we see an angle between the electric field and the current density near the semiconductor-metal interface. This was measured to be approximately $66^{\circ}$. Using Equation 2.8, substituting the semiconducting mobility given in Table 3.1 and the magnetic field of $0.5 \mathrm{~T}$, we 


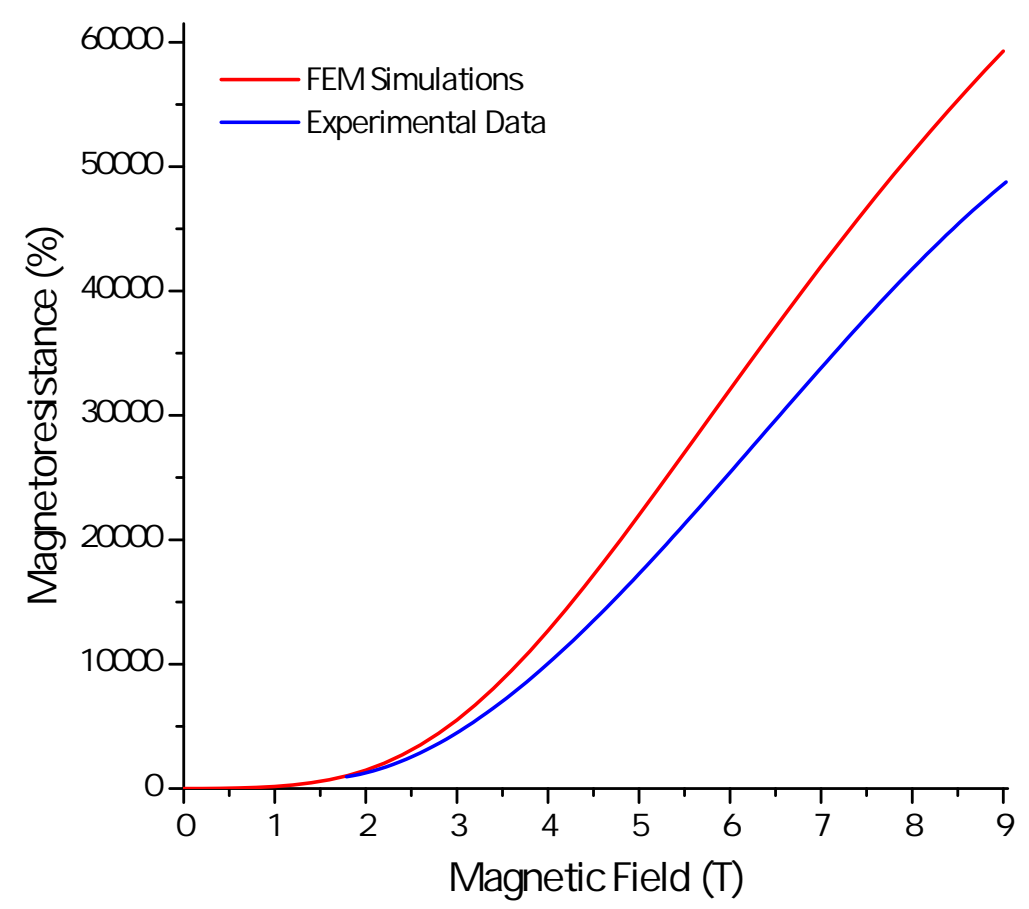

FIGURE 3.20: Magnetoresistance as a function of magnetic field for the FEM model and the corresponding experimental data for the systems of $\mathrm{Lu}$ et al. respectively. The system responsible for these results has a filling factor of $3 / 4$. The device was created using semiconducting graphene and metallic $\mathrm{Pd}$ with the magnetoresistance measured from $0 \mathrm{~T}$ to $9 \mathrm{~T}$. The material parameters used for simulation were taken from the experimental data and can be seen in Table 3.3 .

calculate the Hall angle to be $66.27^{\circ}$ which is remarkably close to the measured value. With a magnetic field of $5 \mathrm{~T}$ the Hall angle is almost $90^{\circ}$ at the interface, as expected (Equation 2.8 produces a Hall angle of $87.48^{\circ}$ ), therefore the current flow is directed around the semiconducting material avoiding the metallic region. This result confirms that the modelling procedure produces the expected results.

The model created here has been used to model other circular geometry experimental EMR systems. This was achieved by simply changing the relative device dimensions and material parameters to those of the experimental systems in question. Figures 3.19 and 3.20 compare the results from the FEM model to experimental data for the results of Suh et al. and $\mathrm{Lu}$ et al. respectively. These results show that an idealised FEM model of such experimental systems can produce a good representation of experimental data. The FEM models tend to overestimate the magnetoresistance which is once again due to the discrepancies between the idealised model and the experimental systems. The appearance of the EMR effect in each case was found to arise due to the same mechanism as observed in Figure 3.16 .

In order to model the experimental results of Suh et al. the FEM model was modified to include the material parameters for these systems (see Table 3.2. Figure 3.19 shows that 
the systems of Suh et al. produce smaller magnetoresistance values than those presented by Solin et al. seen in Figure 3.2 . The main reasons for this are the semiconducting films in the systems of Suh et al. have much lower mobilities (approximately reduced by a factor of four) and the systems are an order of magnitude smaller in dimensions. This essentially reduces the effectiveness of the magnetic field at expelling current from the metallic region. The mobility of a thin film is highly dependent on the growth procedure and growth conditions. For effective EMR devices high mobility films are desirable. In Chapter 5 the effect of the mobility of the semiconducting region is investigated in more detail. In general there is a good agreement between the model and the experimental results in Figure 3.19 considering the model is of an idealised geometry and does not include a resistance at the semiconductor-metal interface.

Figure 3.20 compares the magnetoresistance, as a function of magnetic field, for the experimental data of $\mathrm{Lu}$ et al. and a FEM model of such a system. The system responsible for these experimental results is based upon the same circular EMR geometry as previously used, however the materials in the system's construction are different. The semiconducting region was created from graphene with the metallic shunt created from palladium. This result shows that the EMR effect is a geometrical effect and can be produced in systems of different materials. The influence of the material parameters of an EMR system on the magnetoresistance are investigated in Chapter 5 . The FEM model in this case was created using the material parameters in Table 3.3.

We see that the FEM model shows a very good agreement with the experimental data from such systems, but is once again a slight overestimate of the experimental values. This is attributed to differences between the idealised model and the experimental systems in question. With the use of a model that mimics the specific experimental system (see Figure 3.7) the agreement would be expected to improve. The experimental magnetoresistance was found to be asymmetric with applied magnetic field. This was explained due to the fact that the metallic inclusion in the experimental system was not perfectly centred in the graphene disk (see in Figure 3.7). Lu et al. have produced a very similar FEM model of their own specific system and similarly found a very good agreement with experiment (see Figure 3.8). 


\section{Chapter 4}

\section{Influence of System Geometry}

In this chapter geometrical aspects of EMR systems are considered. The magnetoresistance of a system is made up of two parts: the physical and geometric contributions. The physical contribution originates from the magnetic field dependence of the material parameters. It is responsible for the OMR effect and is generally small for both metals and semiconductors. The geometric contribution arises from the geometry of the system in question; in the EMR effect the geometric contribution is dominant over the magnetoresistance. That is, the OMR that arises from the system's material parameters is smaller than the magnetoresistance caused by the system's geometry. This was apparent in Chapter 3, where the filling factor was found to strongly influence the observed EMR effect ${ }^{9[10] 12}$. In general, a larger filling factor produced higher magnetoresistance values up until very large values. The geometrical component of the magnetoresistance is comprised of the specific shape of the device including the relative dimensions of the metallic and semiconducting regions along with the placement of the contacts. At low field the magnetoresistance is found to increase quadratically with the dimensionless magnetic field. The form of this relation has been presented by Solin et al. and can be seen in Equation $4.1^{\text {r1 }}$.

$$
\operatorname{EMR}(B)=g(B)[\mu B]^{2}
$$

Here, a geometrical factor, $g(B)$, is introduced to scale the magnetoresistance effect, $\mu$ represents the carrier mobility of the dominant carrier and $\mathrm{B}$ is the magnitude of the applied magnetic field. In this chapter, the geometrical aspects of the EMR effect that have been studied previously are reviewed and new geometrical results based on FEM modelling presented. 


\subsection{Previous Geometrical Results}

Since the discovery of the EMR effect in devices of a circular geometry there has been a great deal of research into the properties of EMR devices of an equivalent linear geometry (see Figure 4.2). This linear geometry was produced from the original circular geometry using conformal mapping $14 \mid 15 / 71 / 72$. This technique transforms points from one complex plane onto another in such a way that the local angles between the two planes are preserved. It can be thought of as making a cut along the radius of a circular EMR device and unrolling it to produce a linear device in which the semiconducting and metallic regions are two adjacent rectangles (see Figure 4.1) 14 . The linear device geometry is advantageous in terms of practical applications, since linear devices are more readily fabricated with existing technology. A detailed look at the transformation between the circular and linear EMR devices can be found in Appendix B.

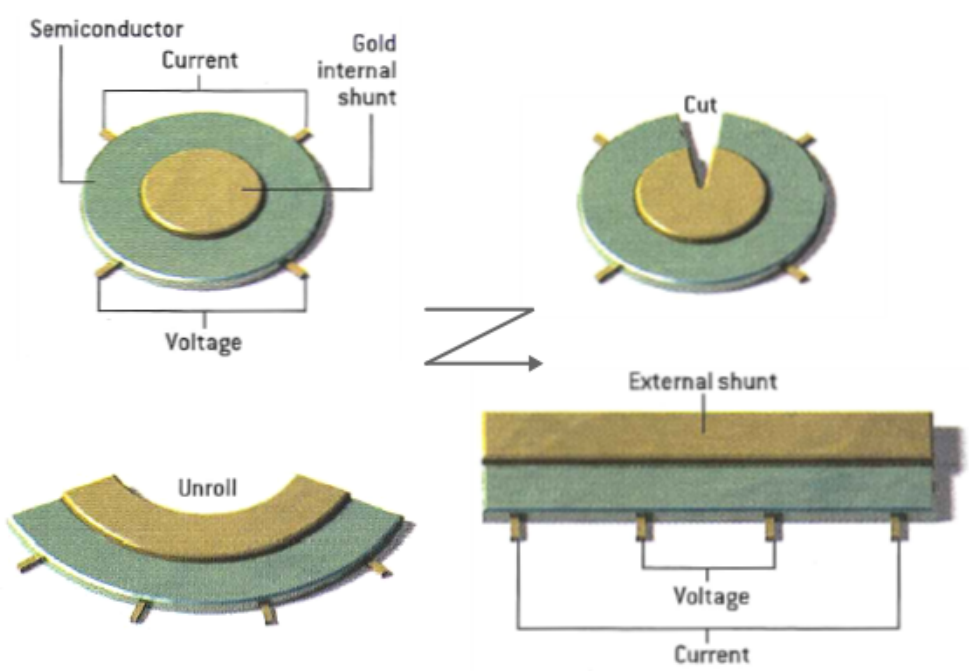

FIGURE 4.1: Diagram outlining the transformation between a circular and linear EMR device. A more detailed analysis of this transformation can be found in Appendix B. (Adapted from reference 14).

Experimental results have shown the existence of the EMR effect in devices with a linear geometry $\frac{1517185}{90}$. Magnetoresistance measurements at room temperature have been published for linear EMR device geometries by Solin et al. where these devices were made using the same materials (InSb and $\mathrm{Au}$ ) as in earlier circular devices. A sketch of a linear geometry EMR device is given in Figure 4.2 along with an electron micrograph image of a real nanoscopic linear EMR device in Figure 4.3 .

Solin et al. reported results from a macroscopic linear EMR device where the magnetoresistance was found to be larger than $100 \%$ in a magnetic field of $0.05 \mathrm{~T}^{15}$. This device has a length of $5 \mathrm{~mm}$ with the width of the conducting region up to $1 \mathrm{~mm}$. With an asymmetric contact configuration on the semiconducting region the magnetoresistance 

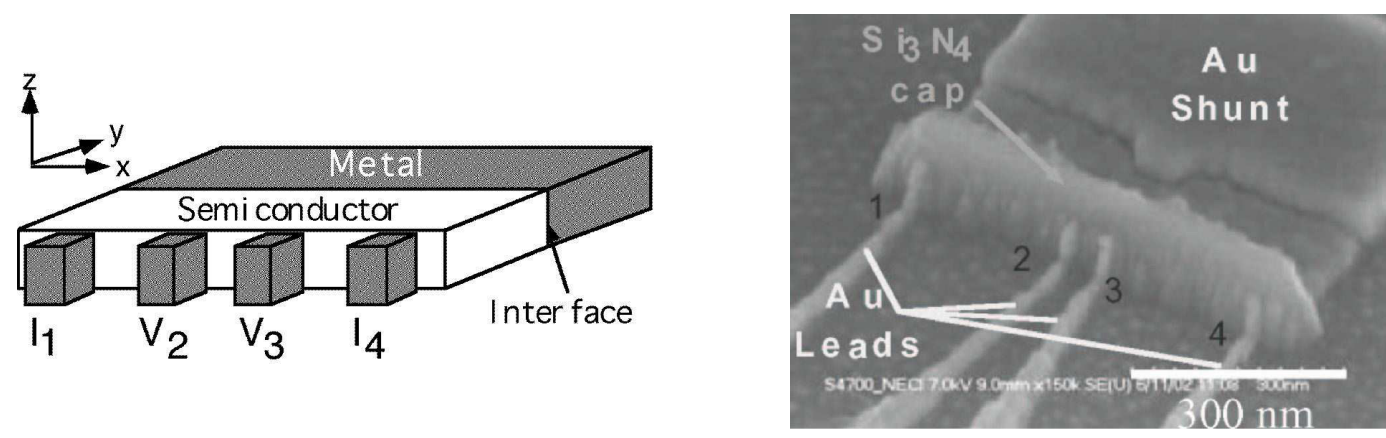

Figure 4.2: A schematic diagram of a linear geometry EMR hybrid configured for a four probe measurement. I and V refer to current and voltage probes respectively. (Reproduced from reference 15).

Figure 4.3: An electron micrograph of a linear geometry EMR hybrid. The semiconducting (InSb) region is capped by $\mathrm{Si}_{3} \mathrm{~N}_{4}$ with a gold metallic shunt and four contacts.

(Reproduced from reference 15).

was also found to be asymmetric. Thus, by using a finite magnetic field as the reference field instead of zero (as normal), the magnetoresistance could be increased. Since a potential application of the EMR effect is in magnetic field sensors used in data storage devices, this macroscopic sensor was required to be scaled down into the nanoscopic regime and operate at low magnetic fields (typically $0.05 \mathrm{~T}$ ). Solin et al. have produced nanoscopic linear EMR devices (see Figure 4.3) that produce room temperature magnetoresistance values of $4.75 \%$ and approximately $165 \%$ at magnetic fields of $0.05 \mathrm{~T}$ and $5 \mathrm{~T}$ respectively 71 . It was found that when a bias field (reference field) of $0.2 \mathrm{~T}$ was used, the achieved magnetoresistance jumped to $35 \%$ at $0.05 \mathrm{~T}$. When crossing into the nanoscopic regime the mean free path becomes larger than the sample size, this means crossing from diffusive to ballistic transport. In the ballistic transport regime the charge carriers undergo very few scattering processes from phonons as they move along the device. The EMR effect relies upon diffusive transport and is dramatically reduced in a ballistic regime. For the material parameters of the circular systems of Solin et al. (see Table 3.1) the mean free path in the metallic region is $0.04 \mu \mathrm{m}$. Therefore, when scaling EMR devices to submicron dimensions the EMR effect will be diminished unless diffusive transport can be maintained. Solin et al. realised that their nanoscopic device overcame this problem since the sidewalls of the device were found to be rippled with an approximate periodicity that enhanced the scattering and thus produced diffusive transport even with a reduced system size ${ }^{14}$.

A similar system, to the linear device of Solin et al. has also exhibited the EMR effect. A semiconductor-metal hybrid structure was formed from Au and a high mobility 2D electron system comprised of InAs/InGaAs ${ }^{85}$. The system created was $200 \mu \mathrm{m}$ long and the width of the semiconducting region varied between 7 and $70 \mu \mathrm{m}$. In these systems the magnetoresistance measurements were carried out at $4.2 \mathrm{~K}$ resulting in the largest magnetoresistance of $115,000 \%$ in a magnetic field of $1 \mathrm{~T}$ in the system with the largest 
filling factor. This value is very large, however this is not so surprising due to the low temperatures. At $4.2 \mathrm{~K}$ the mobility of the $2 \mathrm{D}$ electron system was $62,100 \mathrm{~cm}^{2} / \mathrm{Vs}$, this could also be increased to $149,000 \mathrm{~cm}^{2} /$ Vs using the persistent photo effect where the sample is illuminated by a short light pulse from an LED. In large magnetic fields pronounced Shubnikov-de Haas ( $\mathrm{SdH}$ ) oscillations were observed ${ }^{85}$.

As in the circular EMR devices, graphene has been used as the semiconductor in linear geometry EMR sensors ${ }^{88 / 89}$. In this instance, a gold rectangular shunt and four gold contacts were deposited onto a graphene flake that was located using Raman spectroscopy. The mobility of the graphene flakes is reported to be between $1,000 \mathrm{~cm}^{2} / \mathrm{Vs}$ and $5,000 \mathrm{~cm}^{2} / \mathrm{Vs}$ with measurements carried out at $300 \mathrm{~K}$. No explicit magnetoresistance results were presented in this work, however, the potential difference between the two voltage leads is shown to vary with magnetic field, which means a magnetoresistance effect is present. Graphene may be a good candidate for future EMR devices as its mobility can reach values which are orders of magnitude larger than those in these systems ${ }^{91}$.

The finite element method has been used previously to model linear EMR devices as well as circular geometry devices. In general, FEM models have been shown to give a very

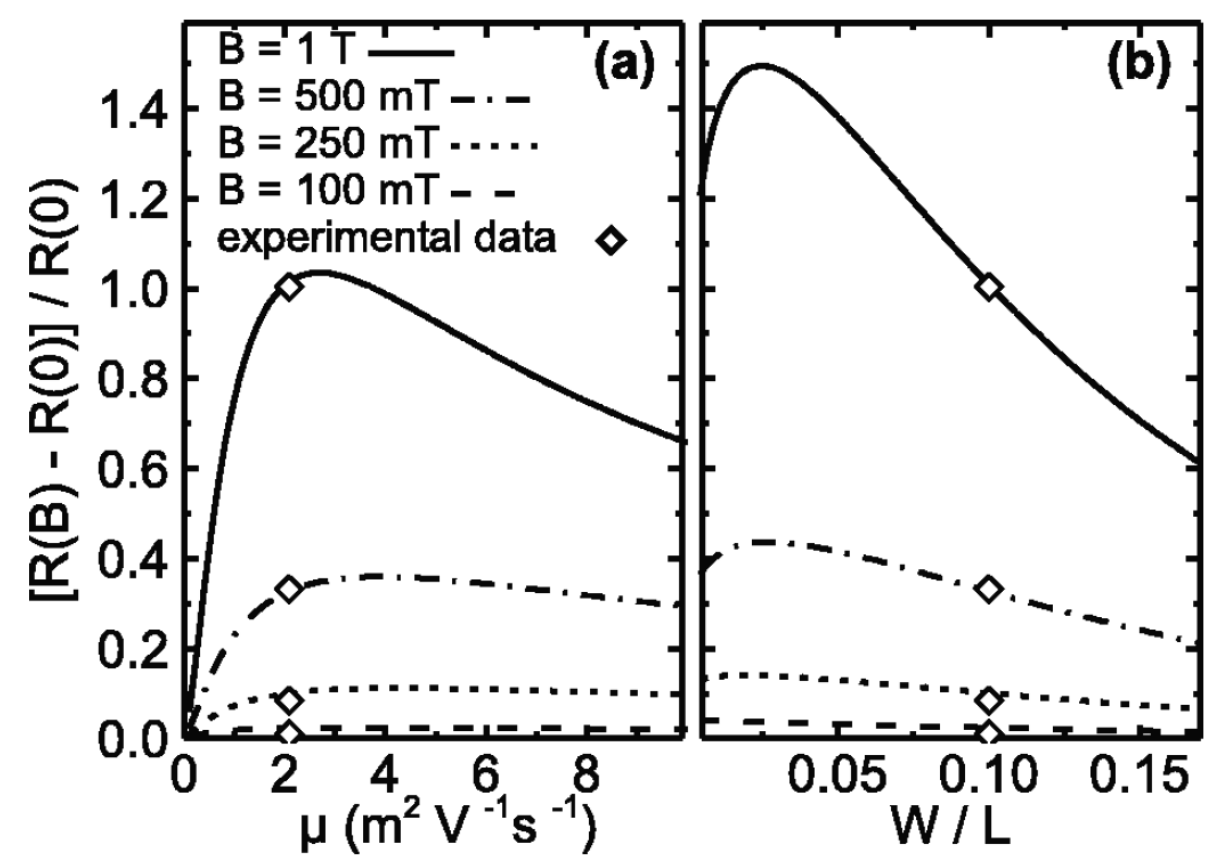

FIgURE 4.4: The magnetoresistance of a linear EMR device with symmetric contact configuration for various values of magnetic field: (a) as a function of electron mobility with W/L fixed at 0.1 (b) as a function of relative semiconductor width $(\mathrm{W} / \mathrm{L})$ for a fixed mobility of $2.09 \mathrm{~m}^{2} / \mathrm{Vs}$. Experimental results are depicted by the open diamonds $(\diamond)$ with the lines representing data from a FEM model. (Reproduced from reference 16). 
good agreement to experimental results $16|2187 / 88| 92 \mid 94$. This can be seen in Figure 4.4 where the FEM model data coincides very well with the experimental data for all values of magnetic field.

One of the strengths of using FEM modelling in such systems is that it allows for the observation of the EMR mechanism in such linear devices 17/1921/93. It has been shown that the mechanism for the expulsion of current from the metallic region in these linear geometry EMR systems is very similar to that observed in the circular EMR

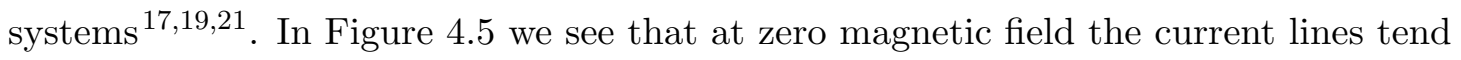
to take a short path, through the semiconducting region at the two current contacts directly into the metallic shunt, producing a low system resistance. At a large magnetic field of $10 \mathrm{~T}$ the current lines no longer enter the metallic region and only flow in the semiconducting region between the current contacts, producing a high system resistance. At intermediate magnetic fields the current flow switches between the two extremes with more of the current lines expelled from the metallic shunt with increasing field.

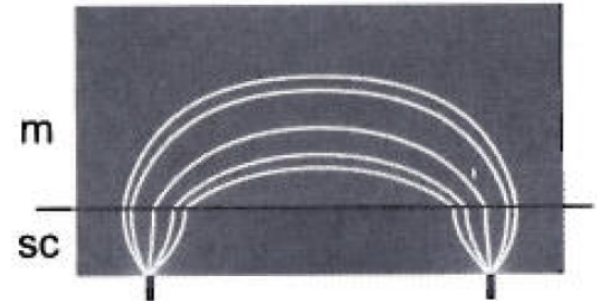

(a) $B=0 T$

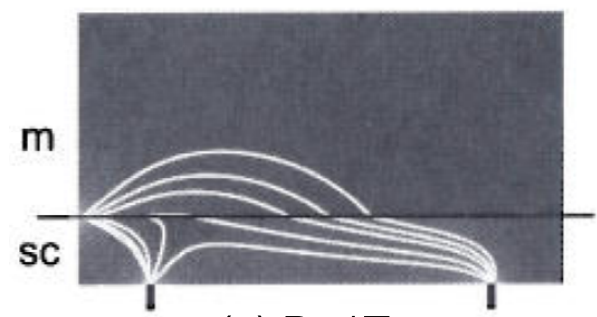

(c) $B=1 T$

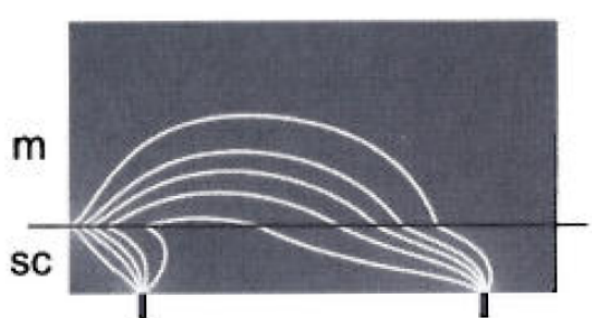

(b) $B=0.5 T$

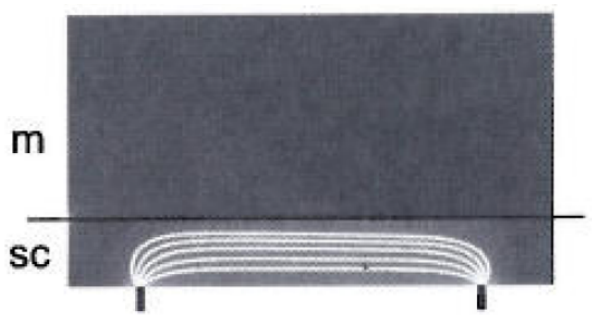

(d) $B=10 T$

FiguRE 4.5: Here, the current flow in a linear EMR device ( $\mathrm{m}$ and sc correspond to the metallic and semiconducting regions respectively) is indicated at four values of magnetic field. The white lines connect points with the same current density between the two indicated current contacts. The mobility of the semiconducting material in this device was $40,200 \mathrm{~cm}^{2} / \mathrm{Vs}$. (Adapted from reference 17).

A specific aspect of the linear EMR device geometry previously investigated is the $\mathrm{W} / \mathrm{L}$ ratio. This usually refers to the ratio of the width of the semiconducting region $\left(\mathrm{W}_{S}\right.$ in Figure 4.14) to the length of the device (L in Figure 4.14) for a fixed width of the metallic region 1611786 . However, the effect of changing the ratio of the width of the metallic region to the length with a fixed width of the semiconducting region has also been considered $15 \mid 20$. Figure 4.4(b) shows the variation of magnetoresistance with changes in $\mathrm{W}_{S} / \mathrm{L}$ for different 
magnetic fields as a result of FEM modelling. This model shows a very good agreement with experimental points, shown by the open diamonds. Larger magnetoresistance values tend to arise for small values of $\mathrm{W}_{S} / \mathrm{L}$. However, for very small values of $\mathrm{W}_{S} / \mathrm{L}$ the magnetoresistance in Figure 4.4(b) is reduced, therefore the optimal value of $\mathrm{W}_{S} / \mathrm{L}$ for such a linear device was 0.025 where the maximum magnetoresistance was observed. In Figure 4.4 (b) the magnetoresistance could be increased from approximately $100 \%$ in the experimental system with $\mathrm{W}_{S} / \mathrm{L}$ of 0.1 , to $150 \%$ for a system with $\mathrm{W}_{S} / \mathrm{L}$ of 0.025 . Also, disappearance of the magnetoresistance was predicted for values of $\mathrm{W}_{S}$ greater than half the distance between the current contacts. In this case the lowest resistance path for the current flow at zero field would not enter the metal at all, but instead flow in the semiconducting region, thus nullifying the EMR mechanism. This is equivalent to the circular EMR device with small filling factors. Here, only a small proportion of the current enters the metallic region as the lowest resistance path for the majority of the current is between the two current contacts avoiding the metal even at zero magnetic field. An increase in $\mathrm{W}_{S}$ for a fixed value of $\mathrm{L}$ and $\mathrm{W}_{M}$ (an increase in $\mathrm{W}_{S} / \mathrm{L}$ ) is equivalent to decreasing the filling factor in a circular EMR device. However, decreasing L (reducing the distance between current contacts) for a fixed value of $\mathrm{W}_{S}$ and $\mathrm{W}_{M}$ (an increase in $\mathrm{W}_{S} / \mathrm{L}$ ) decreases the proportion of current that enters the metallic region at zero field. The direct route from one current contact to the other through the semiconducting material becomes more favourable.

It has been found that the largest magnetoresistance values occur for large values of $\mathrm{W}_{M} / \mathrm{L}^{15120}$. Since it is counterproductive to $\mathrm{W}_{S} / \mathrm{L}$ to increase $\mathrm{W}_{M} / \mathrm{L}$ by reducing $\mathrm{L}$, $\mathrm{W}_{M}$ should be increased to achieve larger magnetoresistance values. For an effective linear EMR device the ratio $\mathrm{W}_{S} / \mathrm{L}$ must be small. Therefore, the distance between current contacts should be much greater than the width of the semiconducting region, and the width of the semiconducting region should be smaller than the width of the metallic region.

Another aspect of the geometry of linear EMR devices previously studied is the position and configuration of the current and voltage contacts. It has been found the EMR effect can be enhanced by changing the relative positions of the current and voltage contacts along the edge of the semiconducting material ${ }^{16}[18 / 20|87| 92$ 94 . Figure 4.6 shows such a linear EMR device where many potential contact positions are indicated 18 . In this arrangement we see two current contacts (labelled I+ and I-) with four proposed positions in which to measure the potential (labelled V1, V2, V3 and V4). A standard contact geometry, similar to the contact configuration in the circular EMR devices (as seen in Figure 3.11) arises when the potential difference is measured between contacts $\mathrm{V} 2$ and V4. This is known as a symmetric IVVI configuration since both voltage probes lie between the two current contacts and the contacts of the system are symmetrical 
about a vertical line in the centre of the device. Investigations into the positions of the contacts tend to fall into two categories. Firstly, the effect of having an asymmetric IVVI contact configuration has been investigated. In this configuration the two voltage probes still lie between the two current contacts but the symmetry about the horizontal line in the middle of the device is lost, this would be the case if the potential was measured between contacts V2 and V3 in Figure 4.6. The second common configuration is called the VIVI (or sometimes IVIV) configuration where only one of the voltage probes is located between two current contacts. A VIVI configuration could be achieved in the system in Figure 4.6 by measuring the potential difference between the voltage probes $\mathrm{V} 1$ and V2.

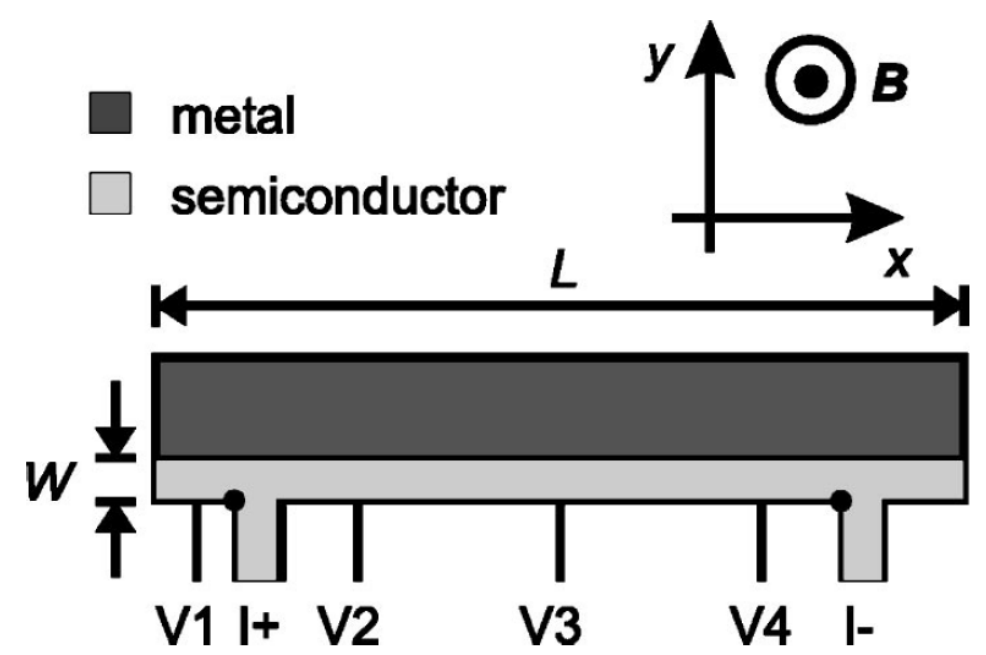

FIGURE 4.6: A sketch of a linear semiconductor-metal hybrid. Here, the current contacts are labelled I+ and I- while four positions of voltage probes are shown. Measuring the voltage with probes V2 and V4 constitutes a symmetric IVVI configuration. Measuring the voltage with probes V2 and V3 is an example of an asymmetric IVVI configuration. Measuring the voltage with probes V1 and V2 constitutes a VIVI configuration.

(Reproduced from reference [18).

In a symmetric IVVI contact configuration the resultant resistance is symmetrical with applied magnetic field, where the same resistance value is obtained for a positive or negative magnetic field of the same magnitude. The first thing to notice about the asymmetric IVVI contact configuration is that the resultant plot of resistance against applied magnetic field is also asymmetric ${ }^{17 / 18|20| 87 / 92}$. This can be observed in Figure 4.7 . This is similar to the asymmetrical magnetoresistance observed by Lu et al. in the circular EMR devices due to the asymmetry in the position of the central conducting region, in the semiconducting disk. In general, the research carried out on the asymmetrical IVVI contact configuration tends to show that the current sensitivity $(\mathrm{dR} / \mathrm{dB})$ and the magnetoresistance of an EMR device can be enhanced over the symmetrical IVVI configuration 20187 . This geometrical enhancement is typically of the order of a factor of 
$2^{20 \mid 87}$. It has been shown the enhancement from the symmetric IVVI contact configuration is largest when the distance between the voltage probes is reduced 20 . This highlights the strong influence the geometry of the contacts in the system has on the magnetoresistance of a linear EMR device.

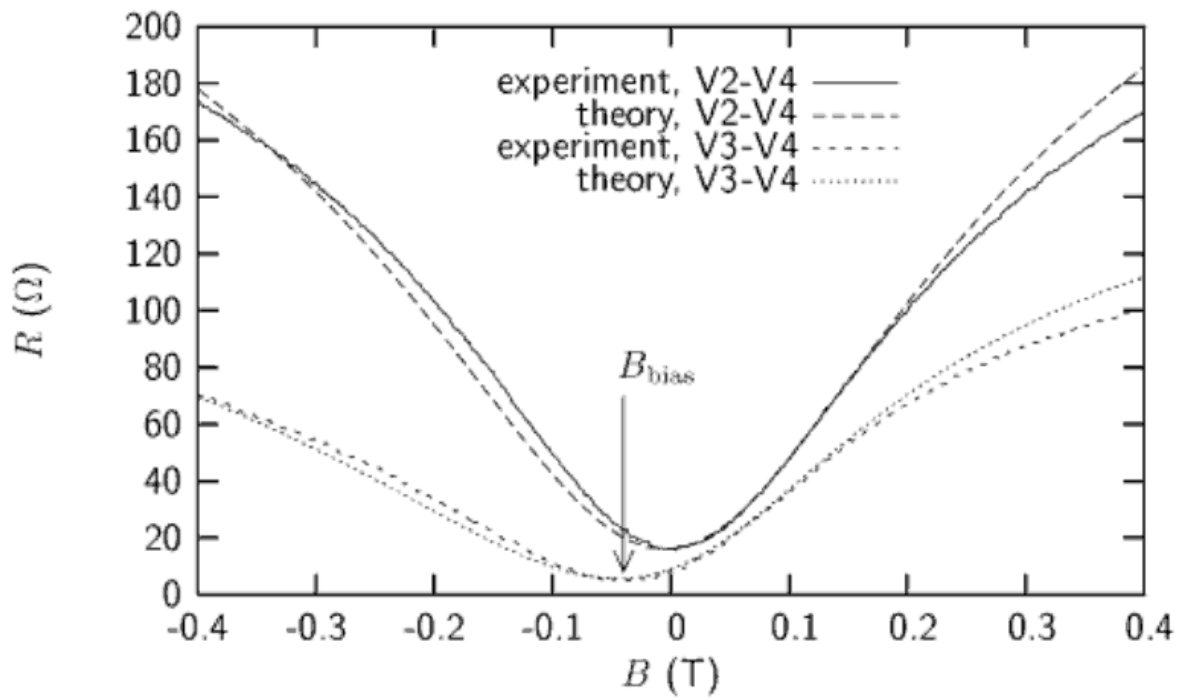

FIGURE 4.7: Resistance as a function of magnetic field (theoretical and experimental data) for a linear semiconductor-metal hybrid device with two contact configurations. The labels have been altered to be consistent with Figure 4.6. The configuration labelled $\mathrm{V} 2-\mathrm{V} 4$ is a symmetric IVVI contact configuration, while the configuration labelled V3-V4 is an asymmetric IVVI contact configuration. $\mathrm{B}_{\text {bias }}$ is the field at which the minimum resistance occurs. Here, $\mathrm{B}_{\text {bias }}=40 \mathrm{mT}$. (Adapted from reference 17).

Investigations into the VIVI contact configuration have also been reported. Here, only one voltage probe is located between the two current contacts $17|18| 92 \mid 94$. In this configuration the magnetoresistance is also asymmetric with magnetic field ${ }^{92}$. The current sensitivity is found to be enhanced over the traditional symmetric IVVI configuration; this enhancement is similarly of the order of a factor of two 18 .

An alternative contact configuration has been investigated by the use of a FEM model ${ }^{93}$. This configuration is similar to the symmetric IVVI configuration with an additional current contact placed between the two voltage probes. This five contact configuration is called the IVIVI configuration where the current is input into the device at the leftmost current contact, while the current is output at both of the other two current contacts (located at the middle and right of the device). The magnetoresistance effect was reported to be enhanced by a factor between 2.4 and 3.7 over that found for a standard IVVI contact configuration. Since the IVIVI contact configuration is not a symmetrical probe configuration (because of the one input current contact and two output current contacts) the potential distribution along the edge of the semiconductor is different in positive and negative magnetic fields, this produces an asymmetric EMR response. This contact 
configuration underlines the significant influence the geometry of the contacts has on the magnetoresistance in a linear EMR device, and that the optimal contact configuration may require more than four contacts in the system.

Considering the EMR effect is dominated by the geometrical component, very little research into the effect of the shape of the semiconducting and metallic regions has been carried out. An EMR device consisting of a square semiconducting region $(3 \mathrm{~mm} \times 3 \mathrm{~mm}$ $\mathrm{InSb})$ with a smaller square metallic region $(2.5 \mathrm{~mm} \times 2.5 \mathrm{~mm} \mathrm{Au})$ deposited on top has been reported ${ }^{95}$. This paper focused on the fabrication method of flash evaporation rather than the effect of a square device on the resultant magnetoresistance. The metallic shunt was not embedded in the semiconducting region and the magnetoresistance results are not nearly as large as the results from Solin et al. in the circular EMR devices. However, it does indicate that EMR devices with different geometries hold great potential for further investigation. Alternatively, it has been proposed that the circular EMR devices could be produced using less Au with the use of a metallic ring instead of a completely solid circle ${ }^{96}$. Here, the outer semiconducting disc contained a metallic ring with semiconducting material on the inside. It was found that devices of this nature exhibited an EMR effect. The reduction in the amount of Au used in the devices containing conducting rings instead of circles, resulted in smaller EMR values. The larger the semiconducting disc in the centre of the metal ring (i.e. the thinner the metallic ring) the more severe the reduction in the magnetoresistance.

\subsection{Geometrically Enhanced Magnetoresistance}

The EMR effect is unlike many other large magnetoresistance effects in that it depends more strongly on the geometrical contribution to the magnetoresistance than the physical one. We have seen that previous studies have investigated some aspects of the geometry of an EMR system, these include varying: the filling factor in circular devices; the ratio $\mathrm{W} / \mathrm{L}$ in linear geometry devices; and the size, position and number of contacts used. However, there has been very little work based upon the shape of the conducting region inside the device. Here, the effect of altering the shape of the metallic region in an EMR device has been investigated. Since the mechanism for the EMR effect relies upon the switching of current paths inside the device, it is reasonable to conclude that the mechanism will be influenced by the shape of the metallic region. We know that the EMR effect is strongly influenced with a change in filling factor, therefore the filling factor was fixed at $\alpha=8 / 16$ for all system geometries with only the shape of the metallic region was varied in each case. The system geometries investigated can be seen in Figure 4.8 . 


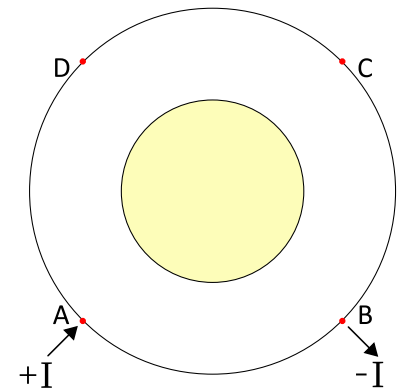

(a)

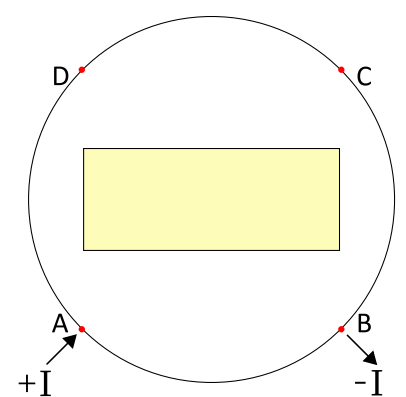

(d)

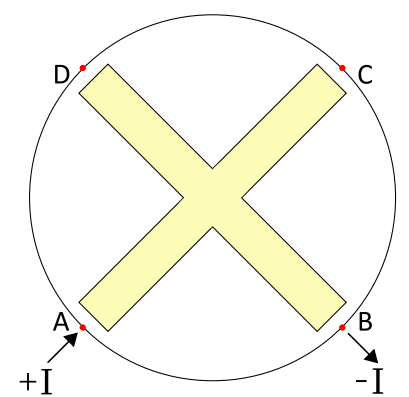

(g)

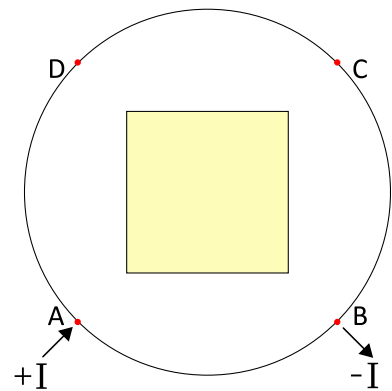

(b)

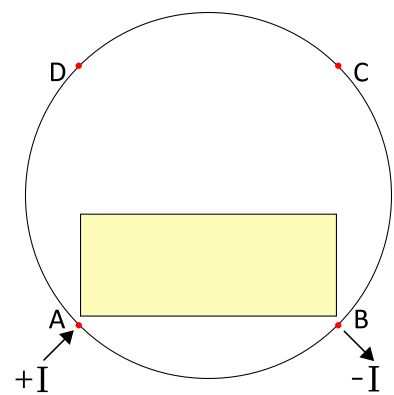

(e)

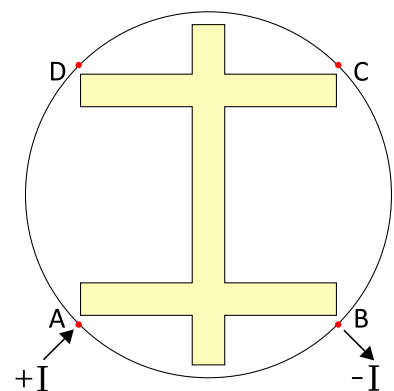

(h)

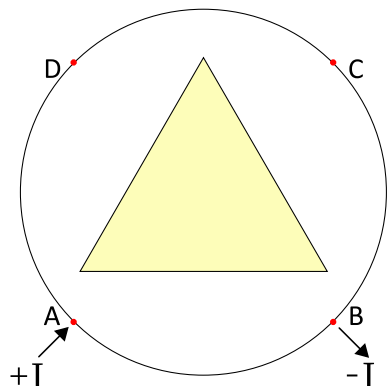

(c)

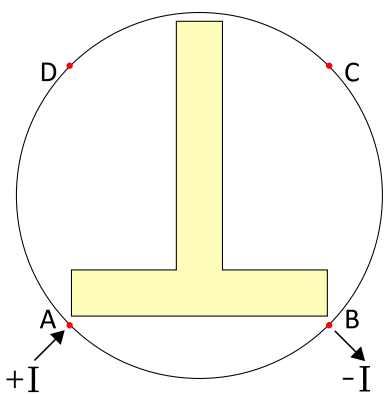

(f)

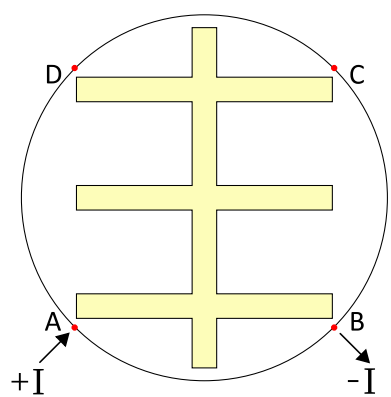

(i)

FIGURE 4.8: EMR system geometries where the shape of the metallic region has been varied while keeping the filling factor fixed $(\alpha=8 / 16)$. These geometries dramatically influence the magnetoresistance values produced in such systems.

These new geometries are compared with the original circular geometry seen in Figure 4.8(a) (as used by Solin et al.) for the same filling factor. The modelling of such systems is identical as for the circular geometry devices seen in Chapter 3 except the shape of the conducting region varied in each case. The material parameters of these systems are given in Table 3.1. The magnetoresistance results for these new geometries are given in Figure 4.9, as a function of applied magnetic field. The 9 lines on this plot correspond to the 9 different system geometries shown in Figure 4.8 .

From Figure 4.9 we see that the geometry of the conducting region has a dramatic influence on the resultant magnetoresistance of the device. We see that the magnetoresistance can 


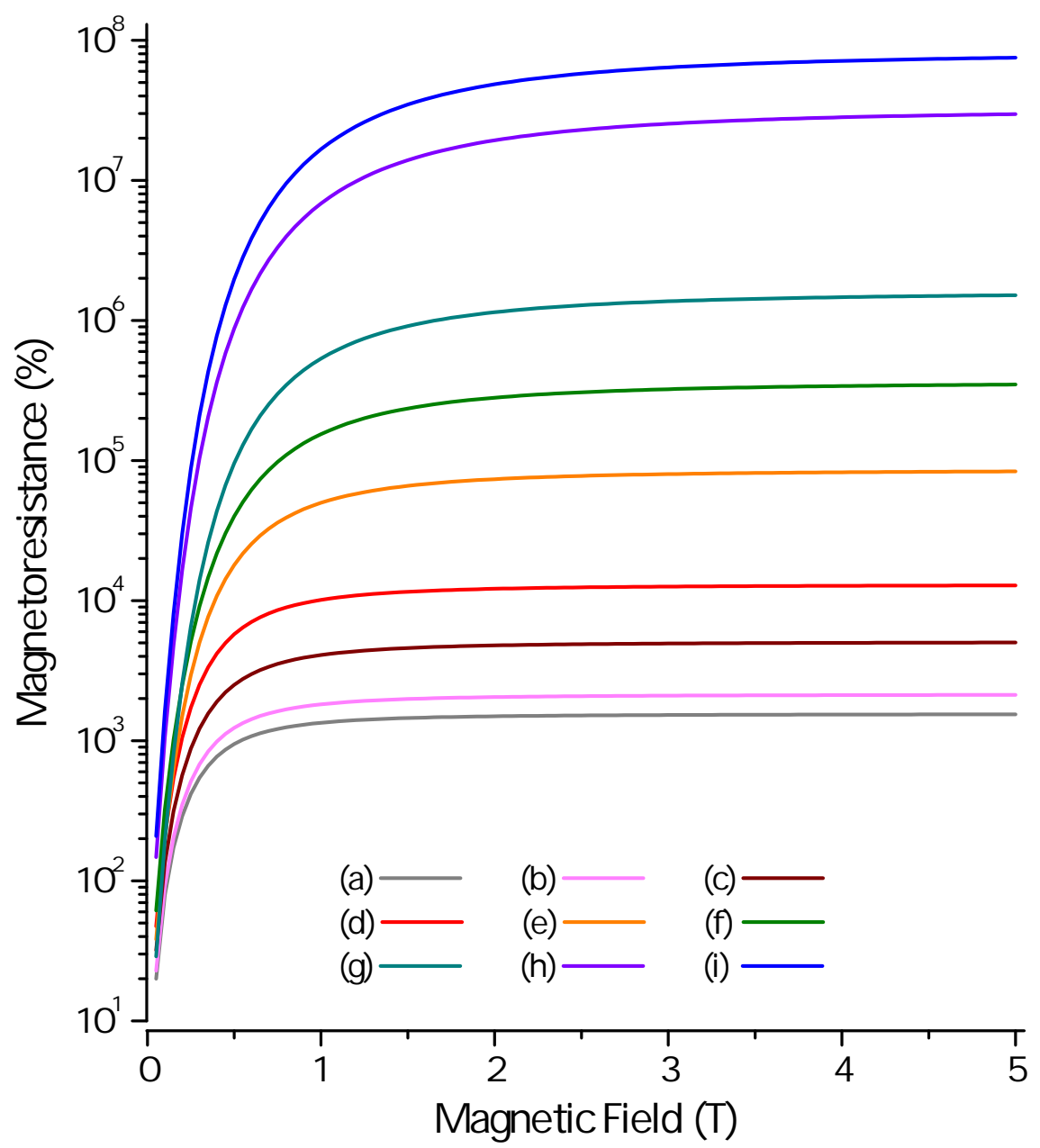

FIGURE 4.9: The magnetoresistance as a function of applied magnetic field for 9 EMR devices (as seen in Figure 4.8) each with a different shaped metallic region for a filling factor of $\alpha=8 / 16$. It is apparent that the shape of the conducting region has a dramatic impact on the magnetoresistance produced in each case.

be enhanced, over the circular geometry, by changing the shape of the metallic region in the device. Here, the device with circular metallic region has the lowest magnetoresistance of all the geometries investigated. Having a circular metallic region, results in a device with the largest distance between current contacts and the semiconductor-metal interface. Devices with metallic regions that move the concentration of metal away from the centre of the device result in larger magnetoresistance values. We also see that the region near the current contacts is very important for enhancement of the magnetoresistance, if we compare the two systems containing a rectangular metallic region in Figure 4.9 (d) and (e). Shifting the metallic region towards the current contacts results in an enhancement of the magnetoresistance by a factor of 6.5. The metallic regions become more branched in the devices seen in Figure 4.9 (f) - (i) and the magnetoresistance increases further. The largest enhancement occurs for the multi-branched structure in Figure 4.8(i) where the magnetoresistance is found to be over four orders of magnitude larger than the 


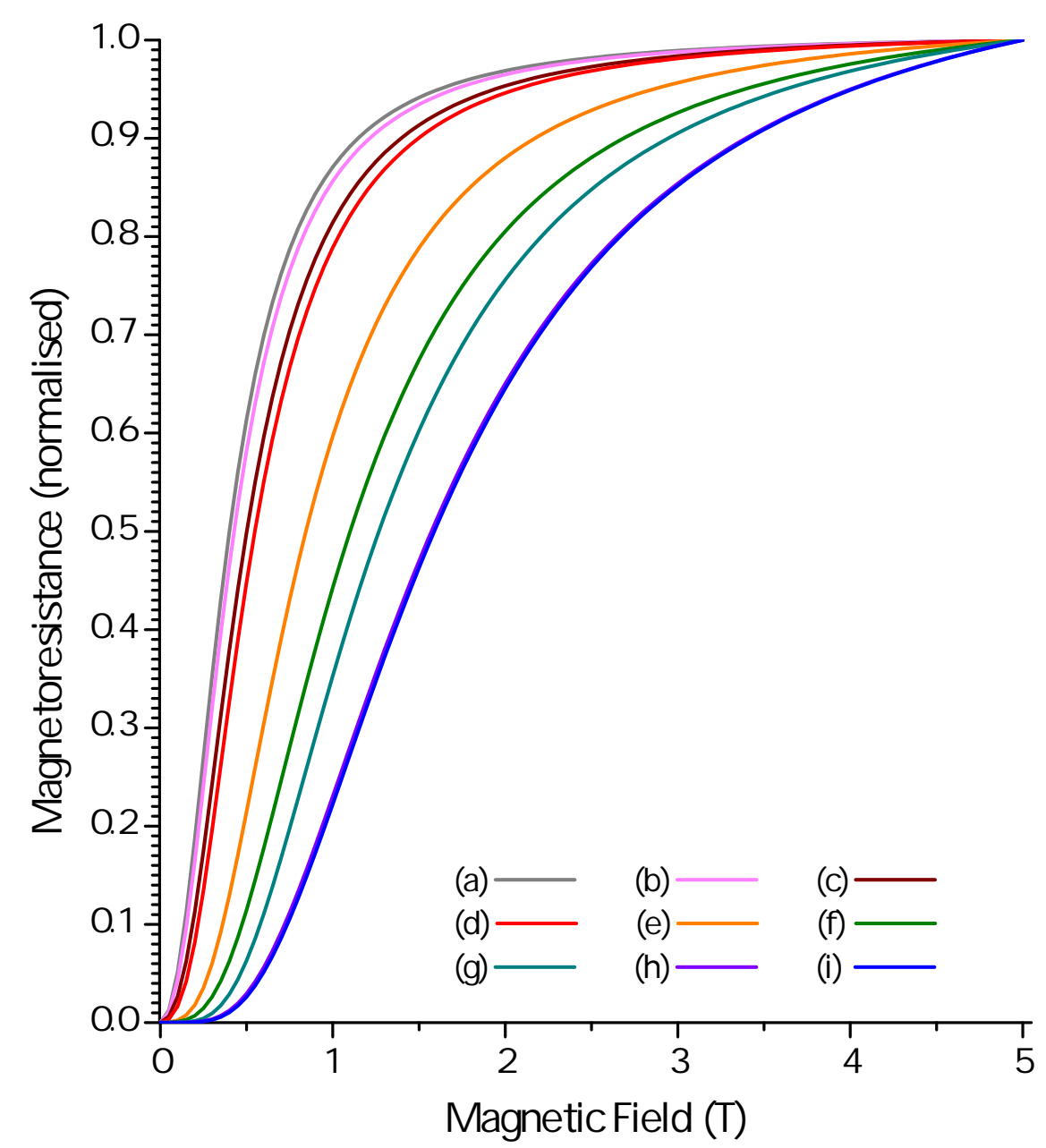

FIGURE 4.10: Normalised magnetoresistance as a function of applied magnetic field for 9 EMR devices (as seen in Figure 4.8) each with a different shaped metallic region for a filling factor of $\alpha=8 / 16$. Here, the functional form of the magnetoresistance is found to be dependent on the system geometry.

circular geometry, at a magnetic field of $5 \mathrm{~T}$ (see Figure 4.11). This is a huge geometrical enhancement for systems with the same filling factor.

In order to test whether the functional form of the magnetoresistance is dependent on the system geometry for each of the 9 geometries seen in Figure 4.8 (as suggested by Equation 4.1), the magnetoresistance has been normalised by its maximum value in each case. Figure 4.10 presents the normalised magnetoresistance for each geometry against magnetic field. Here, the functional form of the magnetoresistance is found to be dependent on the system geometry, with the more complex branched structures requiring a larger magnetic field in order to switch between the low and high resistance states.

The huge geometrical enhancement of the magnetoresistance observed in Figure 4.9 for the multi-branched geometry (Figure 4.8(a)) over the circular geometry device (Figure 4.8(i)) is quantified in Figure 4.11. Here, the geometrical enhancement is seen to be significant at 


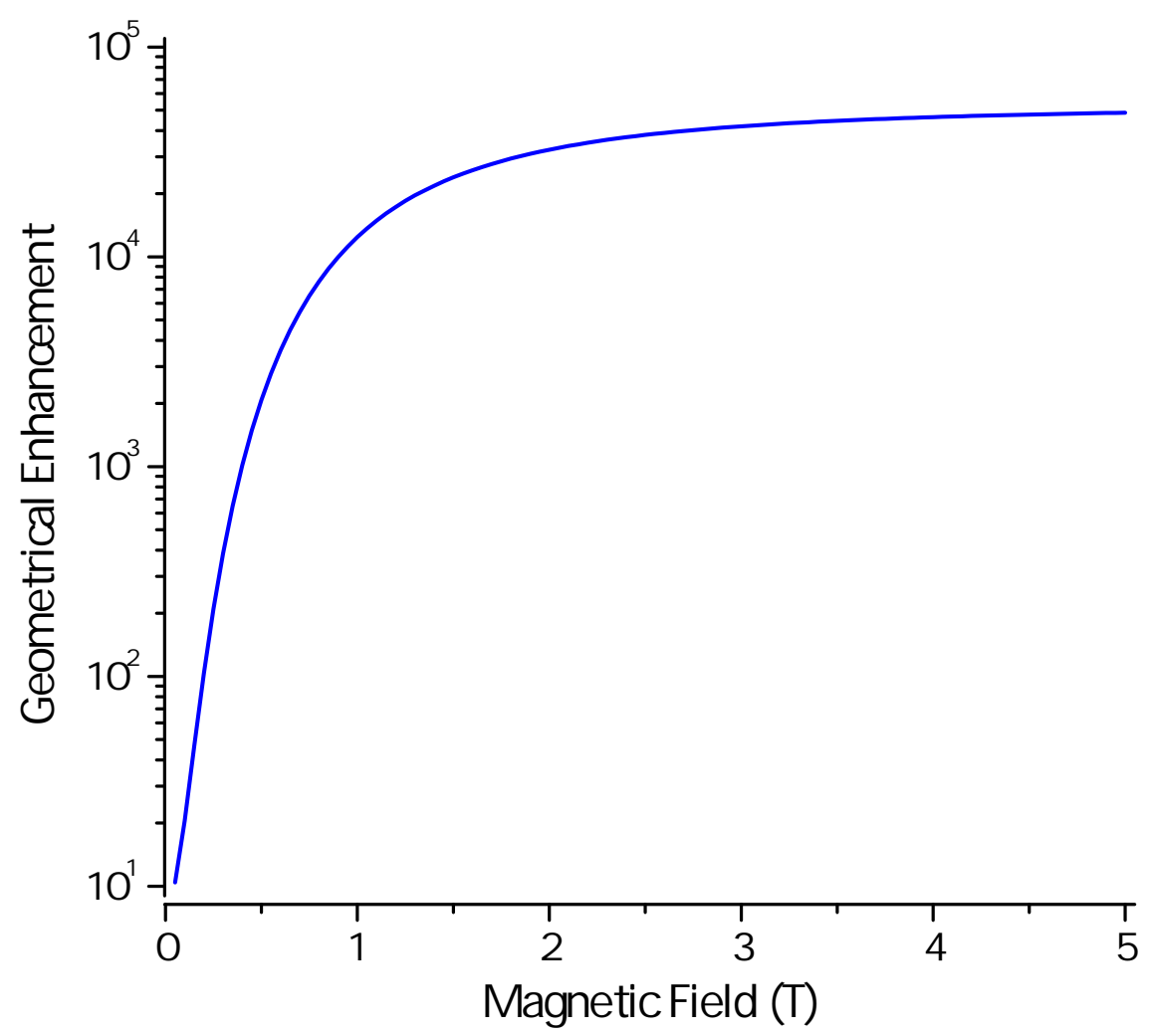

FIGURE 4.11: The factor of geometrical enhancement of the magnetoresistance between an EMR device with a branched metallic region over the circular metallic region (see Figure 4.8 (a) and (i)) as a function of magnetic field. The geometrical enhancement is huge at large magnetic fields reaching over four orders of magnitude.

low magnetic fields, reaching: an order of magnitude at $0.05 \mathrm{~T}$; two orders of magnitude at $0.2 \mathrm{~T}$; and three orders of magnitude at $0.4 \mathrm{~T}$. However, the most dramatic enhancement is observed at high magnetic fields where the enhancement exceeds four orders of magnitude in a magnetic field of $5 \mathrm{~T}$.

In order to see how this multi-branched geometry (Figure 4.8(i)) enhances the magnetoresistance and how the magnetoresistance effect arises, the change in current flow throughout the system for six values of magnetic field was investigated and can be seen in Figure 4.12. Here, we see that the current distribution is more complex and diverse than the one found for systems containing circular metallic droplets (see Figure 3.16). However, in general the same mechanism can be observed. From the current streamlines we see in all six cases the current flow is highly inhomogeneous and changes significantly with magnetic field. Without magnetic field the current direction is always perpendicular to the metallic surface and the majority of the current flows through the metallic region. The new multi-branched system geometry means the low resistance path has an extremely low resistance. The majority of the current flows through the lower horizontal bar (as observed in Figure 4.12(a) where the large horizontal black arrows indicate the largest current flow) with only an extremely small fraction of the current path passing through 


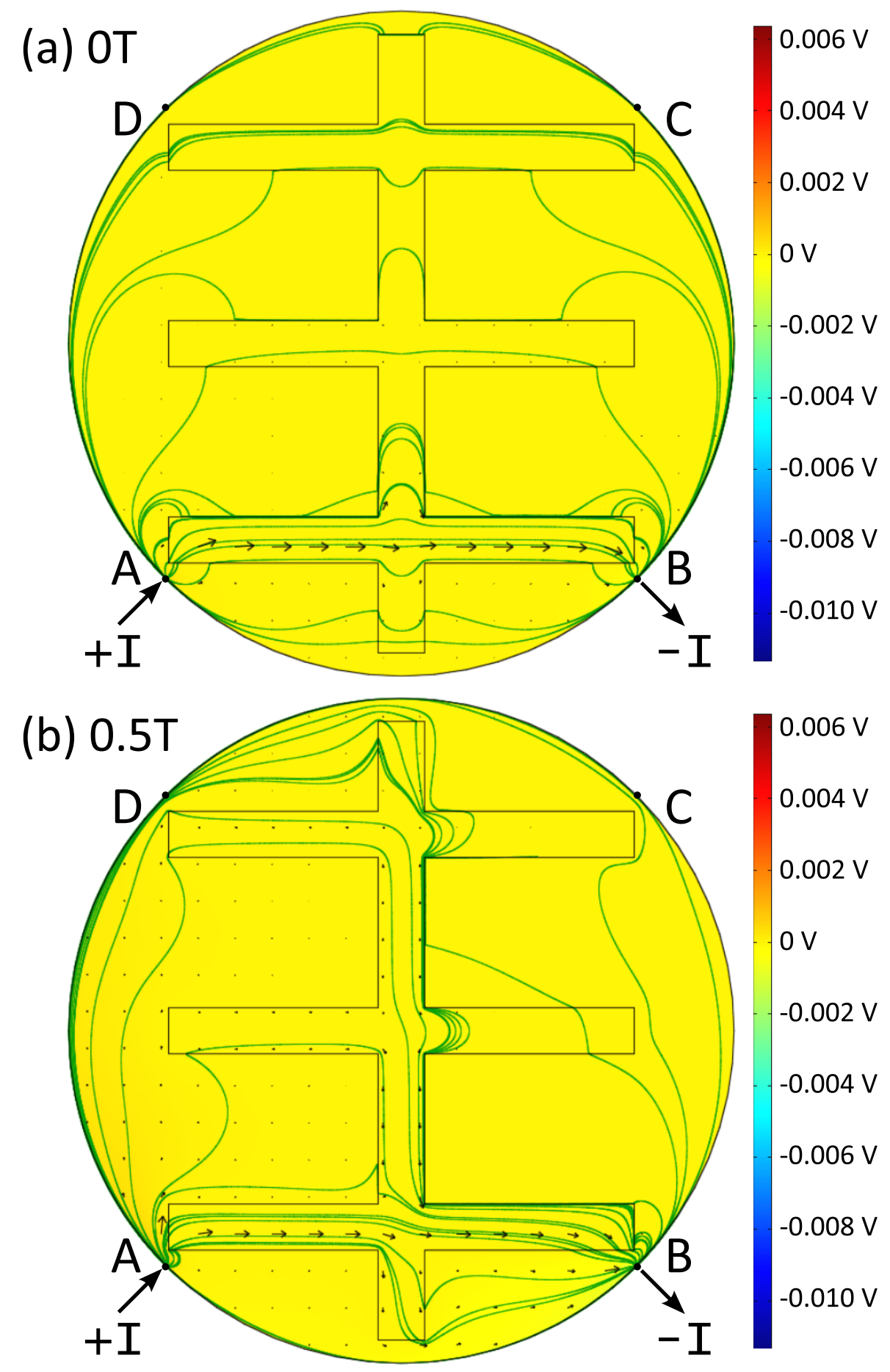

FIGURE 4.12: Visualisation of the current flow and the distribution of electrical potential throughout a modified van der Pauw disk with a multi-branched conducting region $(\alpha$ $=8 / 16)$, produced via modelling in Comsol Multiphysics. Here, the background colour represents the distribution of electrical potential with the coloured bar showing the corresponding voltages (in units of volts). The current density is represented by both the green streamlines and the black arrows. The streamlines connect regions with the same current density, while the arrows show the magnitude and direction of the current flow at various points throughout the system. The six images in this figure represent different applied magnetic fields: (a) 0T; (b) 0.5T; (c) 1T; (d) 2.5T; (e) 4T; and (f) 5T. 


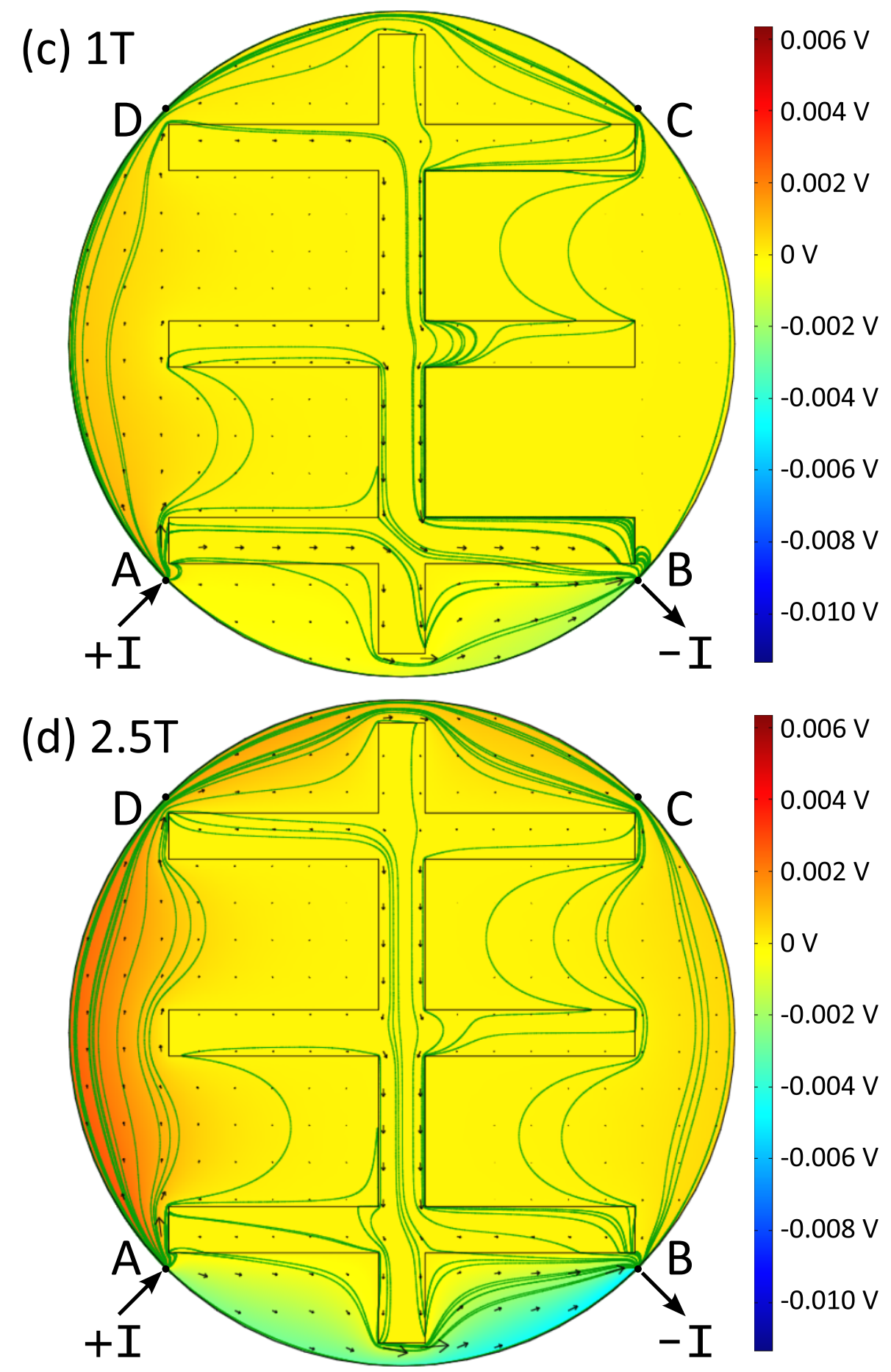

Figure 4.12: (Cont.) Visualisation of the current flow and the distribution of electrical potential throughout a modified van der Pauw disk with a multi-branched conducting region $(\alpha=8 / 16)$, produced via modelling in Comsol Multiphysics. Here, the background colour represents the distribution of electrical potential with the coloured bar showing the corresponding voltages (in units of volts). The current density is represented by both the green streamlines and the black arrows. The streamlines connect regions with the same current density, while the arrows show the magnitude and direction of the current flow at various points throughout the system. The six images in this figure represent different applied magnetic fields: (a) 0T; (b) 0.5T; (c) 1T; (d) 2.5T; (e) 4T; and (f) $5 \mathrm{~T}$. 


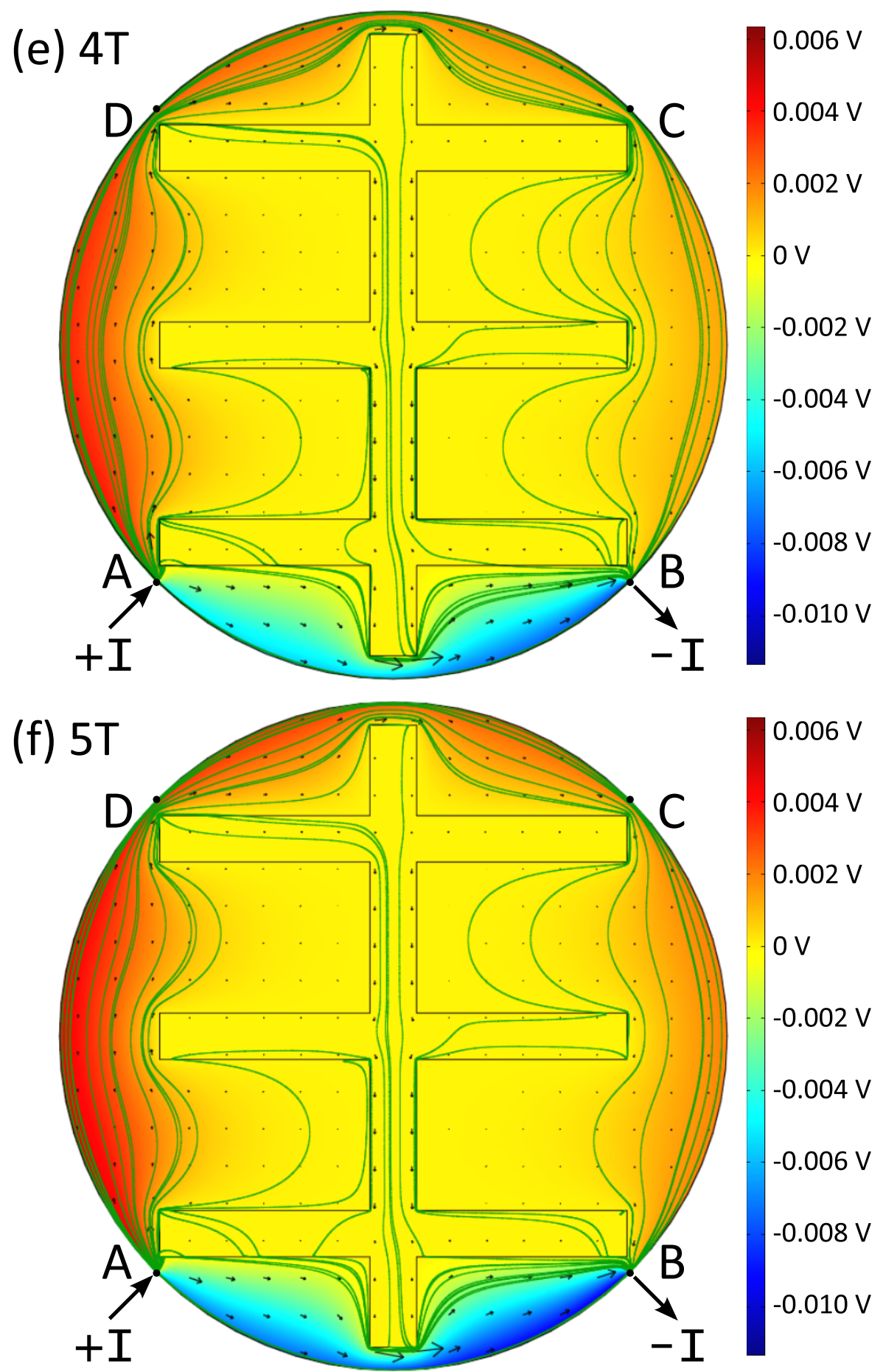

Figure 4.12: (Cont.) Visualisation of the current flow and the distribution of electrical potential throughout a modified van der Pauw disk with a multi-branched conducting region $(\alpha=8 / 16)$, produced via modelling in Comsol Multiphysics. Here, the background colour represents the distribution of electrical potential with the coloured bar showing the corresponding voltages (in units of volts). The current density is represented by both the green streamlines and the black arrows. The streamlines connect regions with the same current density, while the arrows show the magnitude and direction of the current flow at various points throughout the system. The six images in this figure represent different applied magnetic fields: (a) 0T; (b) 0.5T; (c) 1T; (d) 2.5T; (e) 4T; and (f) $5 \mathrm{~T}$. 
semiconducting material. The high field limit (see Figure 4.12(f)) also enhances the magnetoresistance due to the geometry. The application of a magnetic field forces an increasing proportion of the current to flow through the semiconductor (see Figures 4.12(b) to (e)). In large fields the current is tangential to the semiconductor-metal interface, thus is forced to avoid the metallic region. The subsequent path the current takes in the semiconductor is long and becomes extremely narrow at various points. The fact the current is forced along long paths, through narrow channels of semiconducting material, increases the resistance at high field and therefore enhances the magnetoresistance dramatically. However, this multi-branched structure does lead to an increase in the magnetic field at which the magnetoresistance saturates. The geometry in Figure 4.8(a) shows saturation in magnetic fields of the order of $1 \mathrm{~T}$. The multi-branched geometry in Figure 4.8(i) does not show saturation until a magnetic field of approximately 3T. From Figure 4.11 we see that the geometrical enhancement of the magnetoresistance observed is more significant at high magnetic fields. With a magnetic field of $0.4 \mathrm{~T}$ the enhancement is three orders of magnitude, below this the geometrical enhancement decreases rapidly to two orders of magnitude at $0.2 \mathrm{~T}$ and just one order of magnitude at $0.05 \mathrm{~T}$. The reason why the geometrical enhancement is so dramatic is due to the geometry not only increasing the difference in the system resistance between low and high magnetic fields, but also the system resistance at zero field is significantly reduced. This causes a huge enhancement in the magnetoresistance due to the definition given in Equation 2.1.

In order to quantify the switching of current paths from flowing through the metallic region at low field, to flowing through the semiconducting regions at high magnetic field, a vertical cross-section has been taken through the branched system with the current density plotted (see Figure 4.13). Here, the normalised current density is plotted along a vertical cross-section of the disk at low and high fields (0T and 5T). At zero field we see a maximum in the current density occurring between points 3 and 4 along the cross-section. This is consistent with Figure 4.12(a) where the majority of the current flows through the lower horizontal metallic bar. Since a huge majority of the current is flowing through this metallic region, the system's zero field resistance is much lower than for the circular geometry EMR device as seen in Figure 4.8(a). In contrast, at a magnetic field of $5 \mathrm{~T}$ we see the current density has local minima in regions where the horizontal metallic bars are located (between points 3 and 4,5 and 6 and 7 and 8). The maxima in the current density occur between points 1 and 2 and 9 and 10, these correspond to the thin semiconducting channels at the top and bottom of the device. With the current forced to flow through these narrow semiconducting channels the system's resistance is greatly enhanced. Switching between the two regimes occurs with the application of a magnetic field. At intermediate fields current flows through both semiconducting and 


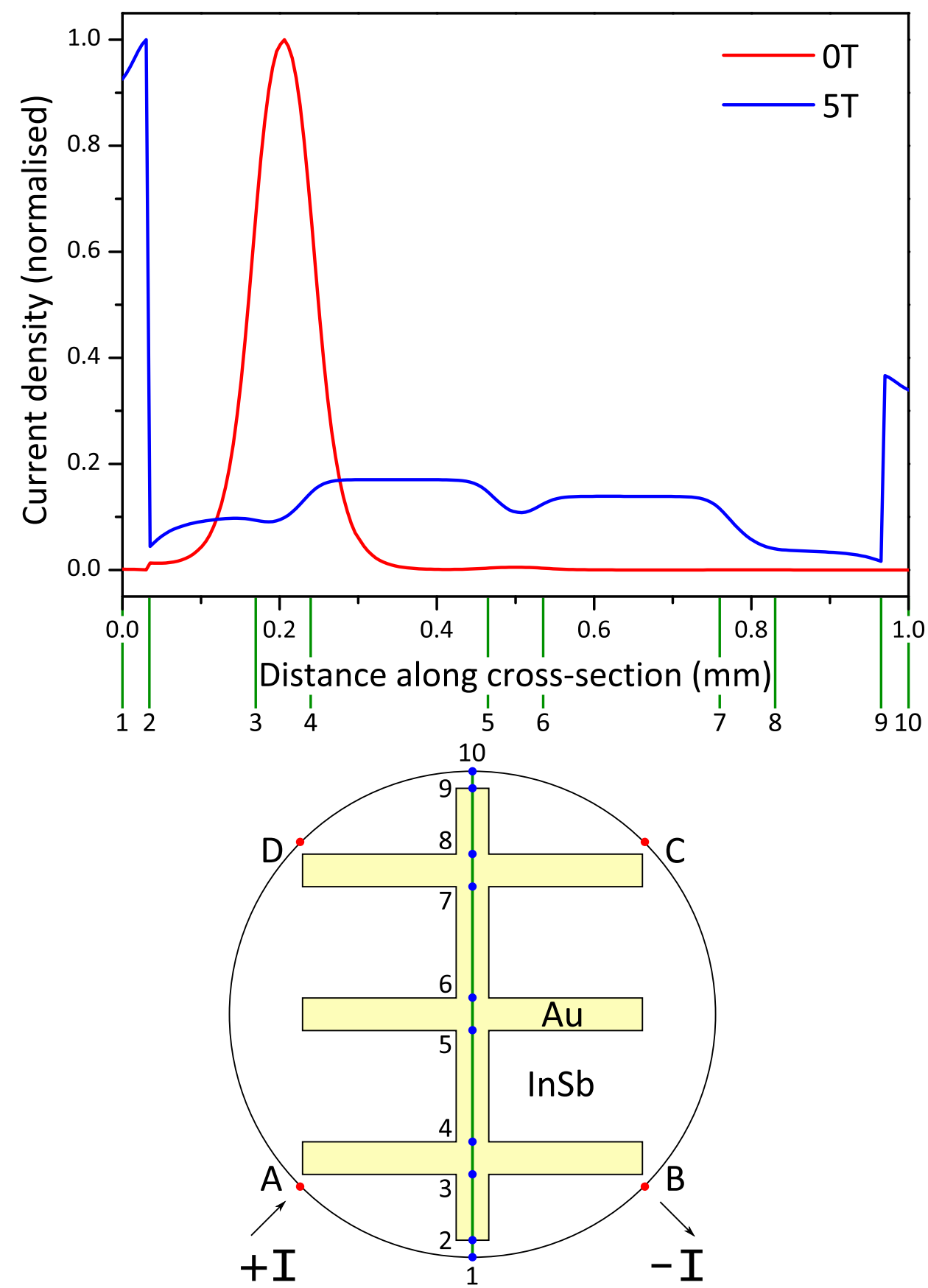

FiguRE 4.13: A cross-section showing the variation in current density (normalised) along the vertical line throughout the system for a multi-branched EMR device (as seen in Figure $4.8(\mathrm{i}))$. The points 1 to 10 on the cross-section correspond to points 1 to 10 on the $\mathrm{x}$ axis of the plot. 
metallic regions. An increase in magnetic field causes a larger fraction of the current to be expelled from the metal and flow through the semiconducting region.

The multi-branched geometry presented here may not be the optimal geometry in order to achieve a large EMR effect. However, it demonstrates the geometry of the metallic region has a huge influence on the resultant magnetoresistance and tailoring the design can lead to vast enhancements to the magnetoresistance. This geometrical enhancement could be very significant for applications of EMR systems whereby other important parameters of EMR devices could be compromised in conjunction with this multi-branched geometry in order to still achieve large EMR effects. For example, semiconducting materials with a lower mobility (e.g. silicon) could be used to produce EMR devices. This would be very beneficial for practical devices since silicon is a semiconductor with well established fabrication methods and techniques. This would allow EMR devices to be produced on a large-scale and at a reduced cost. The multi-branched geometry could be used to achieve larger magnetoresistance effects than circular geometry devices that have a larger filling factor. Therefore, the production of EMR devices based on this multi-branched structure would require less Au.

\subsection{Linear EMR Device Geometry}

Conformal mapping has been shown to convert circular geometry EMR devices (where the metallic shunt is internal in the system) into linear geometry devices (where the metallic shunt is external to the system). This linear EMR device should exhibit the same magnetoresistance mechanism as seen in the circular geometry counterparts. A detailed description of such mapping is presented in Appendix B. In this section a generic linear EMR device geometry is investigated in order to determine how the EMR mechanism occurs in such devices. The model presented could be used as a base for future investigations into specific features of these linear devices or to verify experimental results from a specific system. This would be achieved by adapting the dimensions and material parameters of this model. The model geometry used in this section is presented in Figure 4.14 .

In Figure 4.14 we see the same features as in the circular EMR devices. The system has a semiconducting region to which four contacts are attached, two of these contacts are for current input and output while the other two measure the resulting potential difference. Adjacent to the semiconducting region there is a metallic shunt. The magnetic field is applied in the same manner as in the circular devices, that is, perpendicular to the plane of the device. The device used in this section was investigated in the magnetic field range from 0 to $5 \mathrm{~T}$. The material parameters for the semiconductor (InSb) and the metal 


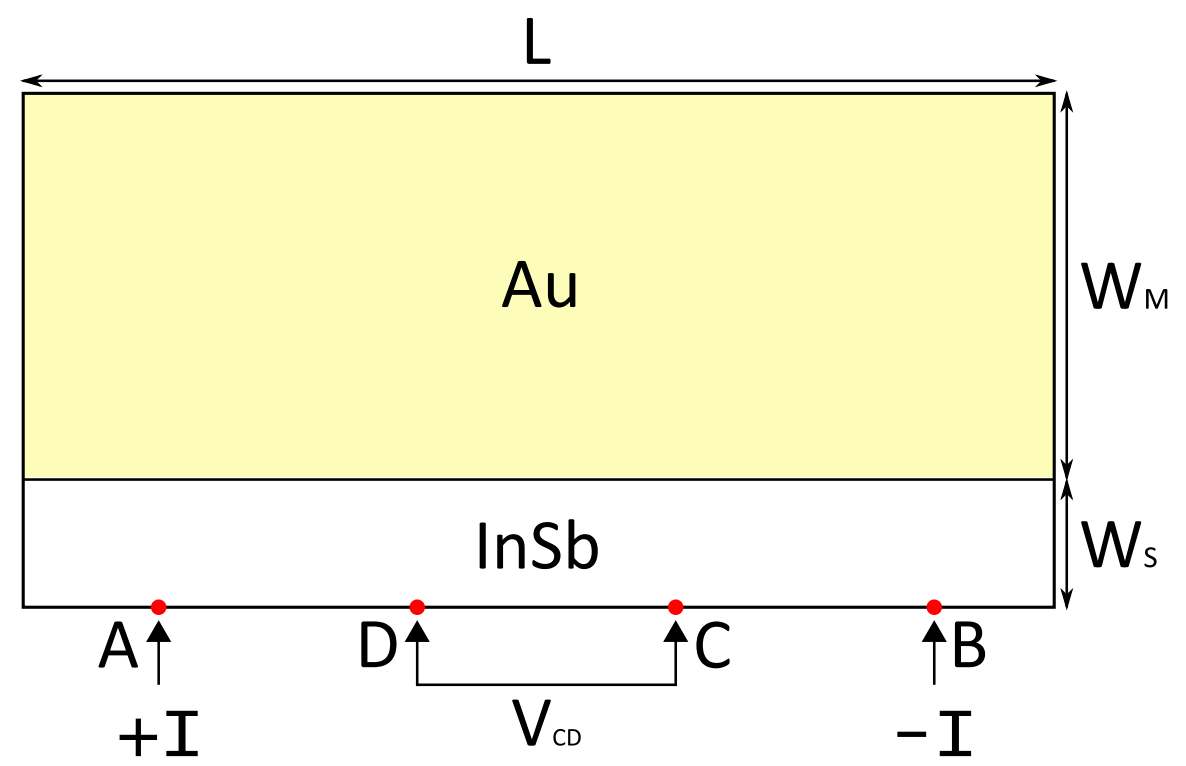

Figure 4.14: System geometry of a linear EMR device. Conformal mapping has shown that equivalent linear devices can be produced from circular geometry devices. This device has similar features to those of the circular devices as seen in Figure 4.8(a). Here, we see a semiconducting region with four contacts placed along the outside of the device. In this geometry the metallic region is said to be external as opposed to internal in the circular devices.

$(\mathrm{Au})$ are the same as in the circular devices by Solin et al. which are given in Table 3.1 . The system shown in Figure 4.14 has the following dimensions: length $(\mathrm{L})=3 \times 10^{-3} \mathrm{~m}$; width of metallic region $\left(\mathrm{W}_{M}\right)=11.25 \times 10^{-4} \mathrm{~m}$; and width of semiconducting region $\left(\mathrm{W}_{S}\right)=3.75 \times 10^{-4} \mathrm{~m}$. Contact $\mathrm{A}$ is positioned $\mathrm{L} / 8$ from the left-hand side of the device. Similarly, contact B is positioned L/8 from the right-hand side of the device. Contact D is positioned $3 \mathrm{~L} / 8$ from the left-hand side of the device, similarly contact $\mathrm{C}$ is positioned $3 \mathrm{~L} / 8$ from the right-hand side of the device. This means $75 \%$ of the device is made from metal, giving a filling factor $(\alpha)$ of 0.866 . This linear device is found to exhibit a large magnetoresistance. Figure 4.15 shows the magnetoresistance as a function of applied magnetic field for the linear device shown in Figure 4.14. Here, the magnetoresistance reaches over $3,000 \%$ in a $5 \mathrm{~T}$ magnetic field.

The magnetoresistance found in this device is smaller than the effect found in a circular device of corresponding filling factor. In order to explain this reduction in magnetoresistance we need to first understand how the EMR mechanism arises in this linear geometry device, therefore it is necessary to look at the current flow throughout the system. Figure 4.16 shows the variation in current throughout the device at 6 values of magnetic field (0T, $0.5 \mathrm{~T}, 1 \mathrm{~T}, 2.5 \mathrm{~T}, 4 \mathrm{~T}$ and $5 \mathrm{~T})$.

Without magnetic field (Figure 4.16(a)) the current appears to flow from contact A 


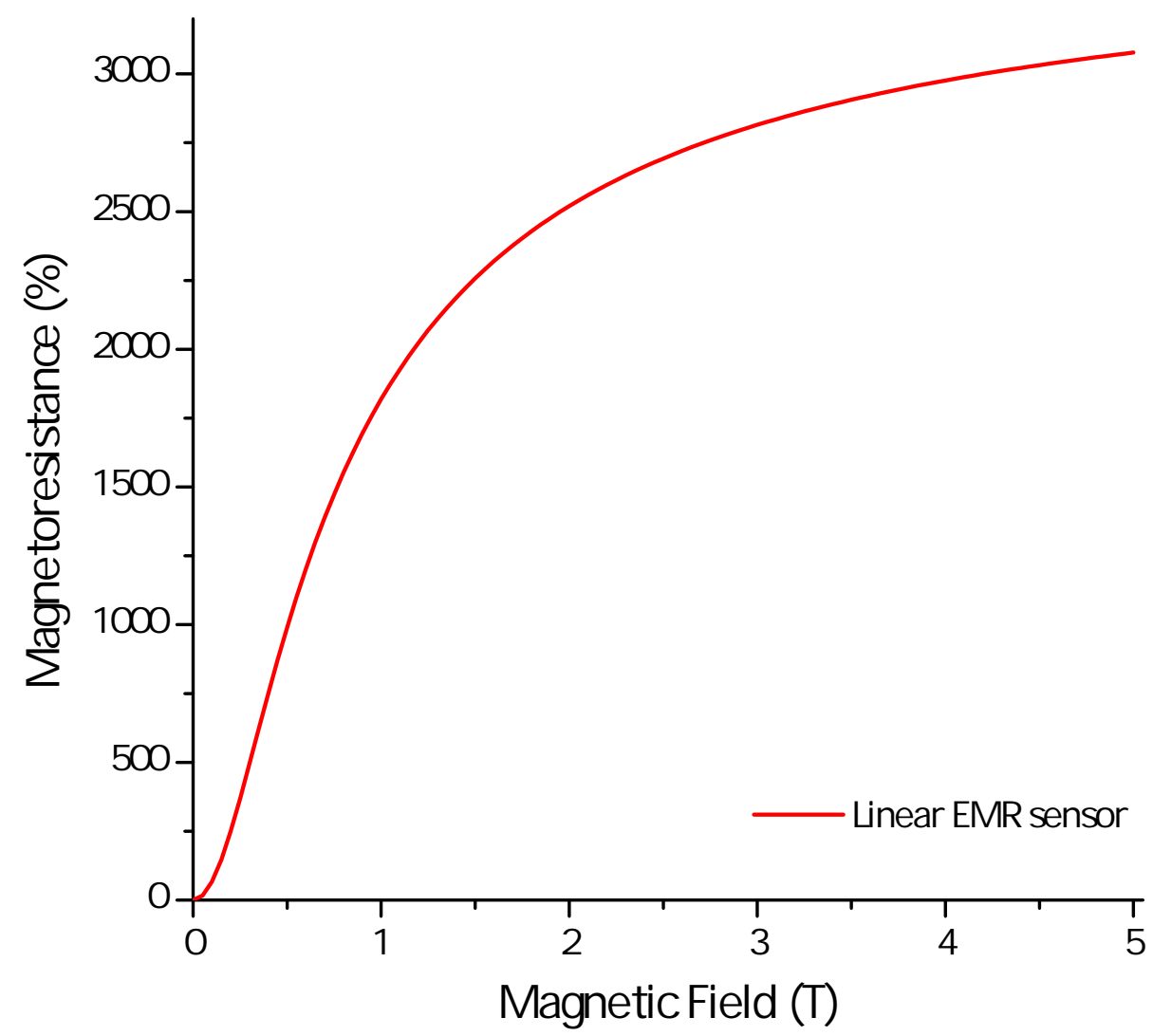

FIGURE 4.15: Modelled magnetoresistance as a function of applied magnetic field for a linear EMR device. The system responsible for these results has a filling factor of 0.866 (see Figure 4.14) and material parameters given in Table 3.1 .

towards the metallic shunt. The majority of the current flows through the metallic region before exiting the system at contact $\mathrm{B}$. The current appears to cross between the semiconductor and the metal at right angles to the interface. At zero field the Hall angle is zero and the current flow is parallel with the electric field lines, therefore current flows into the metallic region. This results in a very low system resistance at zero field since the metallic region is over 2,000 times more conductive than the semiconducting region.

The application of a large magnetic field of $5 \mathrm{~T}$ (Figure 4.16(f)) forces a huge majority of the current flow to be confined to the semiconducting channel and the current flow inside the metallic region almost appears to have vanished. In this high field regime the system's resistance is significantly greater than in the low field regime where the majority of the current flowed through the metallic region. The magnetic field acts to switch the current flow, from mainly through the metal at low fields, to predominantly through the semiconductor at high fields. This is very similar to the EMR mechanism observed in the circular geometry devices, but not equivalent. In Figure 3.16(f) we see that the current is forced to flow around the circular metallic region along two paths; either over (passing contacts $\mathrm{C}$ and D) or under (directly between contacts A and B). However, in the linear device geometry (see Figure $4.16(\mathrm{f})$ ) we see that the main current flow in the 


\section{(a) $0 \mathrm{~T}$}

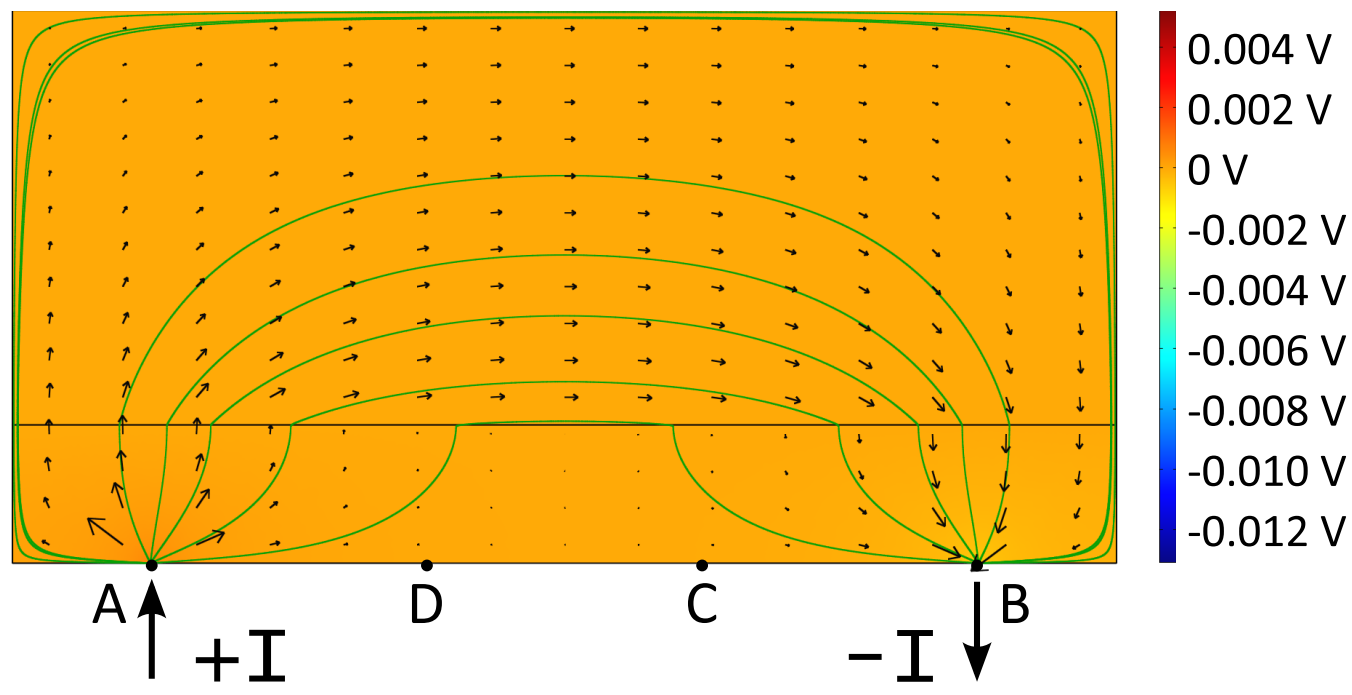

(b) $0.5 \mathrm{~T}$

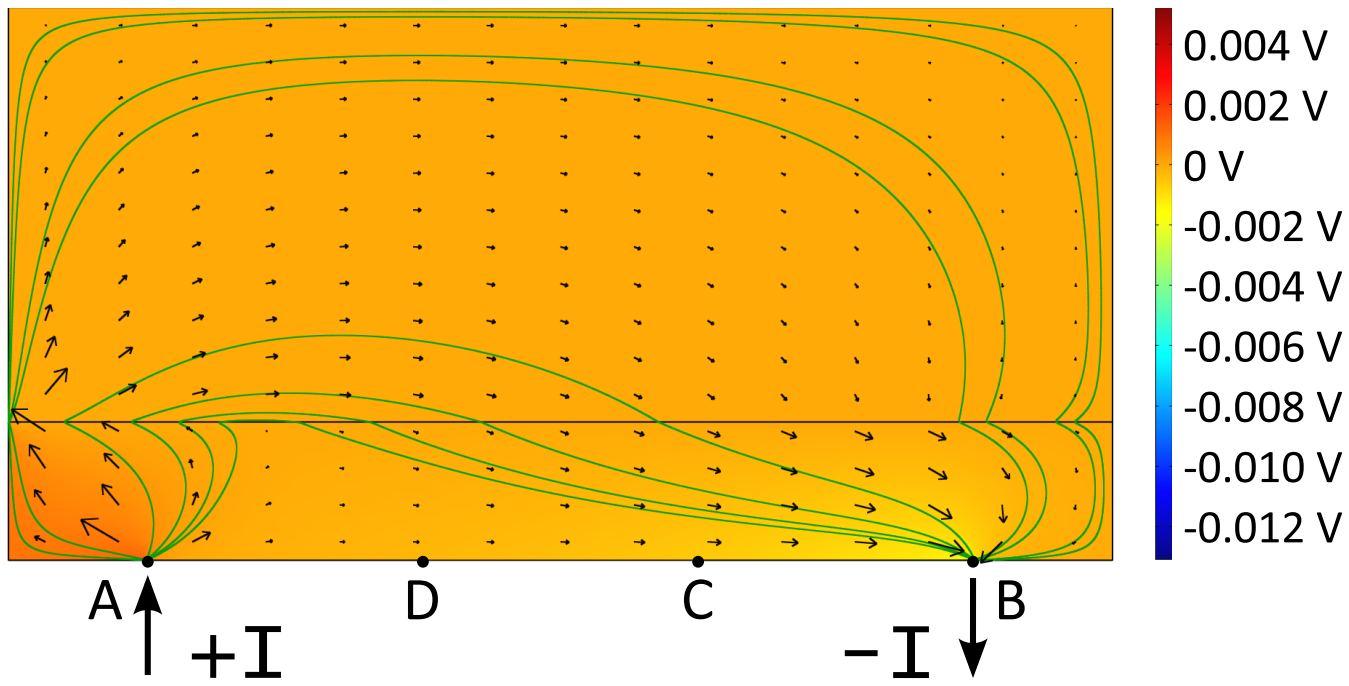

FIGURE 4.16: Visualisation of the current flow and the distribution of electrical potential throughout a linear EMR device $(\alpha=0.866)$, produced via modelling in Comsol Multiphysics. Here, the background colour represents the distribution of electrical potential with the coloured bar showing the corresponding voltages (in units of volts). The current density is represented by both the green streamlines and the black arrows. The streamlines connect regions with the same current density, while the arrows show the magnitude and direction of the current flow at various points throughout the system. The six images in this figure represent different applied magnetic fields: (a) 0T; (b) $0.5 \mathrm{~T}$; (c) $1 \mathrm{~T} ;$ (d) $2.5 \mathrm{~T}$; (e) $4 \mathrm{~T}$; and (f) $5 \mathrm{~T}$. 


\section{(c) $1 \mathrm{~T}$}

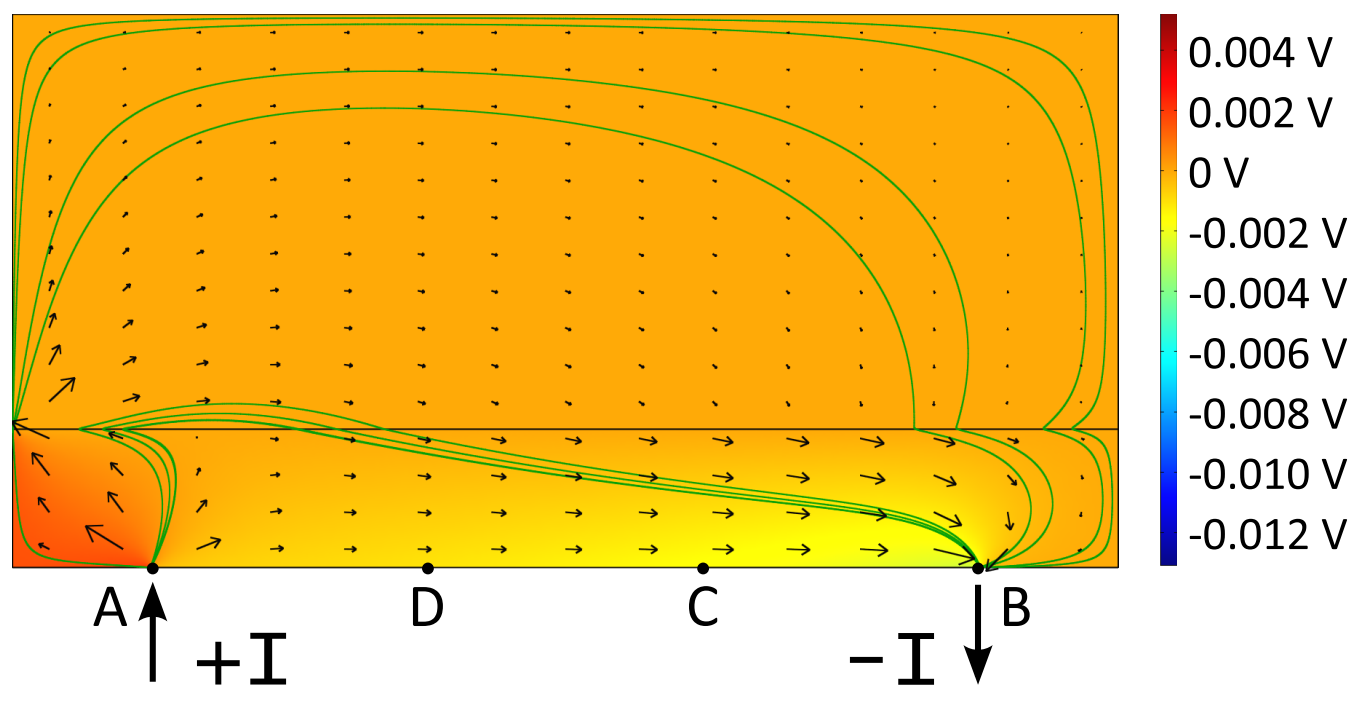

(d) $2.5 \mathrm{~T}$

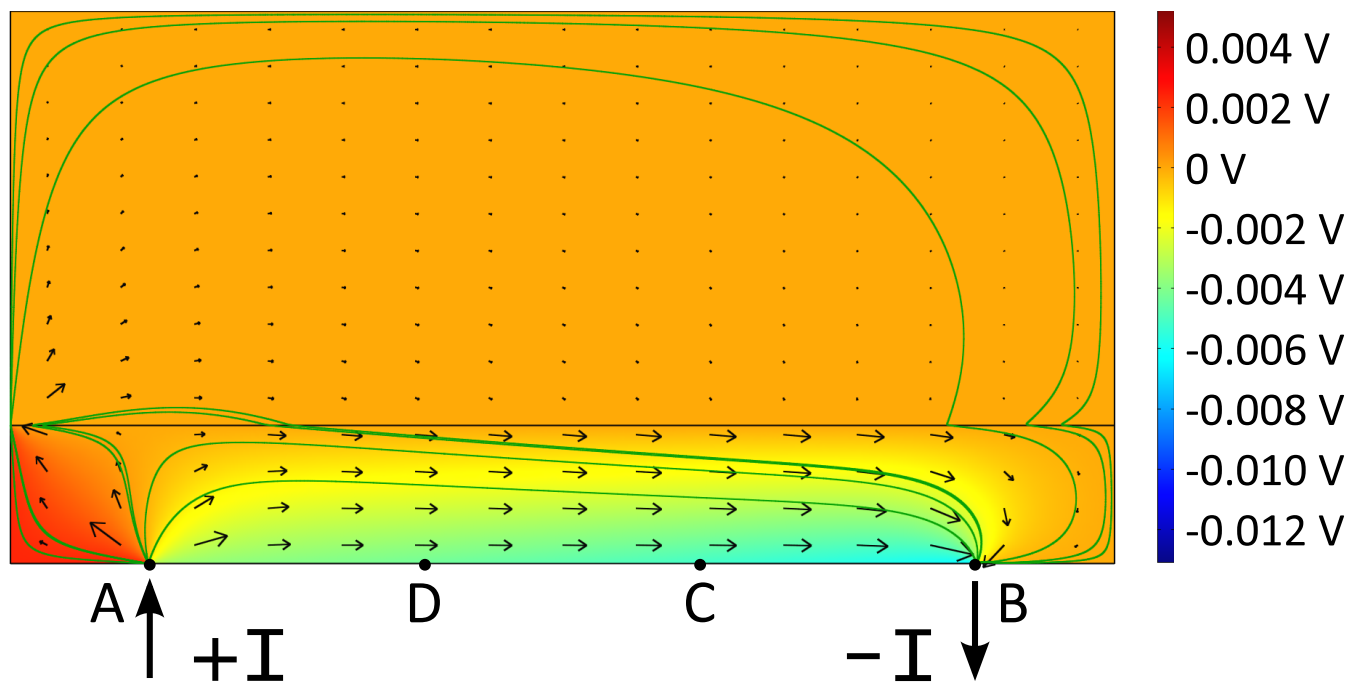

FiguRE 4.16: (Cont.) Visualisation of the current flow and the distribution of electrical potential throughout a linear EMR device $(\alpha=0.866)$, produced via modelling in Comsol Multiphysics. Here, the background colour represents the distribution of electrical potential with the coloured bar showing the corresponding voltages (in units of volts). The current density is represented by both the green streamlines and the black arrows. The streamlines connect regions with the same current density, while the arrows show the magnitude and direction of the current flow at various points throughout the system. The six images in this figure represent different applied magnetic fields: (a) 0T;

(b) $0.5 \mathrm{~T}$; (c) $1 \mathrm{~T}$; (d) $2.5 \mathrm{~T}$; (e) $4 \mathrm{~T}$; and (f) $5 \mathrm{~T}$. 


\section{(e) $4 \mathrm{~T}$}

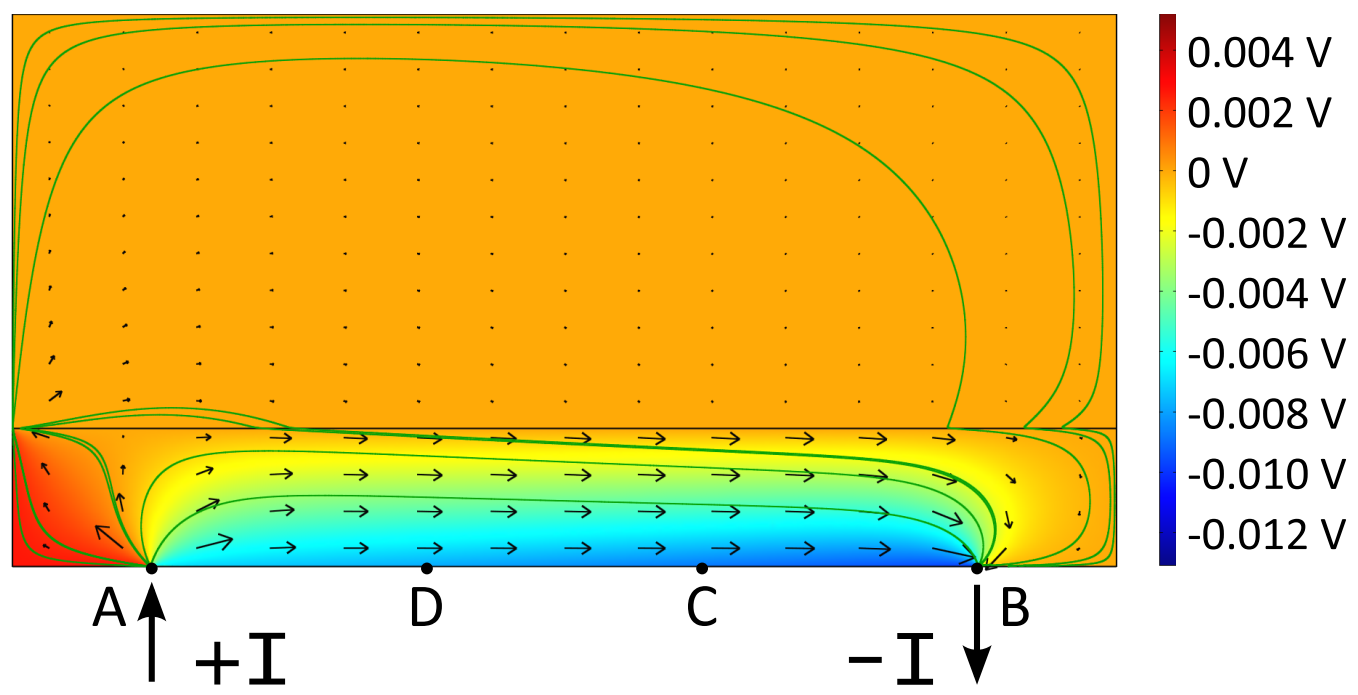

(f) $5 \mathrm{~T}$

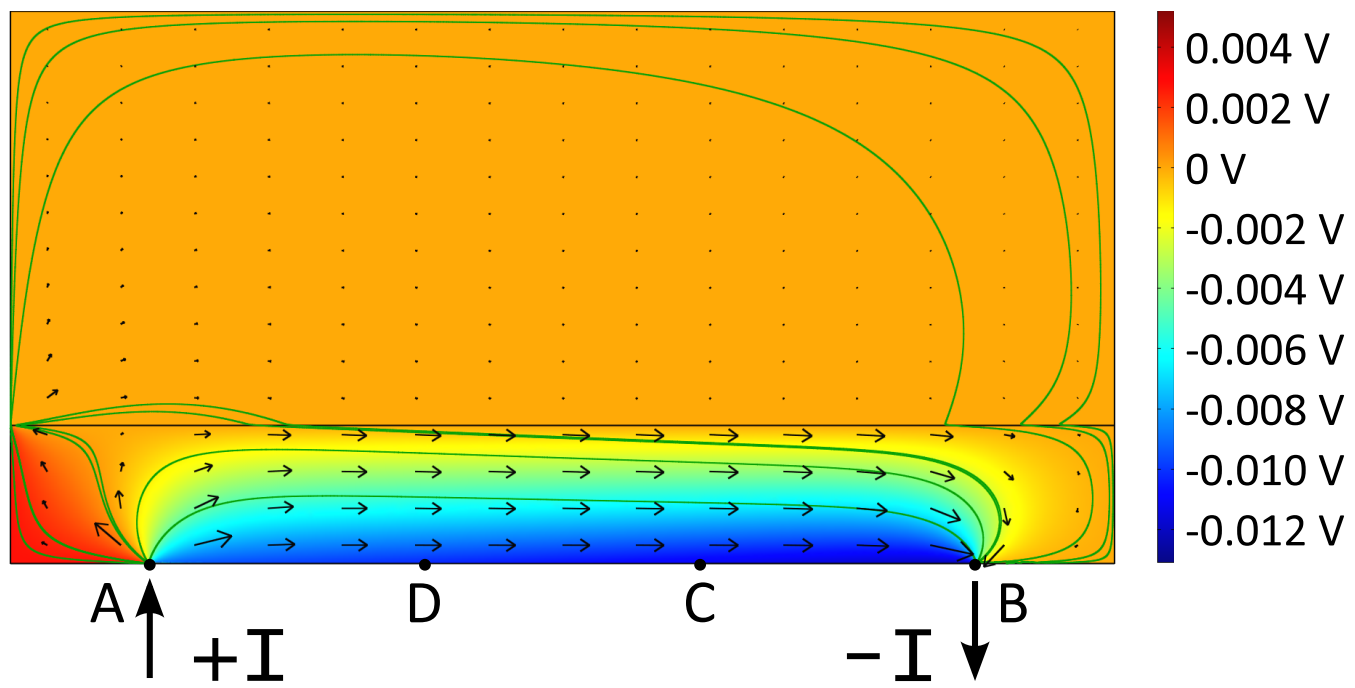

FiguRE 4.16: (Cont.) Visualisation of the current flow and the distribution of electrical potential throughout a linear EMR device $(\alpha=0.866)$, produced via modelling in Comsol Multiphysics. Here, the background colour represents the distribution of electrical potential with the coloured bar showing the corresponding voltages (in units of volts). The current density is represented by both the green streamlines and the black arrows. The streamlines connect regions with the same current density, while the arrows show the magnitude and direction of the current flow at various points throughout the system. The six images in this figure represent different applied magnetic fields: (a) 0T;

(b) $0.5 \mathrm{~T}$; (c) $1 \mathrm{~T}$; (d) $2.5 \mathrm{~T}$; (e) $4 \mathrm{~T}$; and (f) $5 \mathrm{~T}$. 
semiconducting region at high magnetic field occurs between contacts A and B (passing contacts $\mathrm{C}$ and $\mathrm{D}$ ). In this linear geometry the second current path does not exist, since when the current meets the left hand edge of the device it cannot directly reach contact $\mathrm{B}$ and is forced into the metallic region at the semiconductor-metal interface. This is a direct consequence of the cut created when transforming between the two device geometries via conformal mapping.

This feature causes a decrease in the system resistance at high magnetic field and therefore reduces the magnitude of the EMR effect. Additionally, the linear device created when conformal mapping is used on a circular EMR device geometry (see Figure 3.11) is not equivalent to the linear device presented here (see Appendix B for more details). These factors result in a reduction of the magnitude of the EMR effect observed in the linear device geometry over the circular device geometry for the same filling factor, as seen in Figure 4.15 .

At intermediate magnetic fields the transition between the low and high field cases does occur, with varying degree, as in the circular geometry devices. Even at a small magnetic field of $0.5 \mathrm{~T}$ a proportion of the current flow is forced to flow through a larger region of semiconductor. In Figure 4.16(b) the main current flow appears to be from contact A into the metallic region, then the current re-enters the semiconducting region approximately halfway along the device (between contacts D and C). In Figure 4.16(c) the magnetic field of $1 \mathrm{~T}$ has significantly increased the current flowing (from left to right) in the semiconducting region and therefore reduced the current flowing in the metallic region. Figures 4.16.d) and (e) show a system where the majority of the current is flowing through the semiconducting region and the magnetoresistance has begun to saturate. From Figure 4.15, saturation of the magnetoresistance appears to be approaching at approximately 3T. The expulsion of current from the metallic region in high field can be further seen when looking at the current profile along a cross-section of the system as seen in Figure 4.17.

In Figure 4.17 we see that without a magnetic field the current density peaks between points 2 and 3 along the cross-section, corresponding to inside the metallic region. The current flow in the semiconducting region (between points 1 and 2) is very small. This creates a low field regime of low system resistance. The application of a strong magnetic field causes the maximum current flow to arise in the semiconducting region (between points 1 and 2) with the current flow in the metallic region dramatically reduced. This plot reinforces the EMR mechanism in such linear device geometries. The cross-section is similar to that found for the circular EMR devices if only part of the cross-section is considered. By only considering the current plot between points 1 and 3 in Figure 3.17 . a very similar pattern is seen for the linear device geometry. 

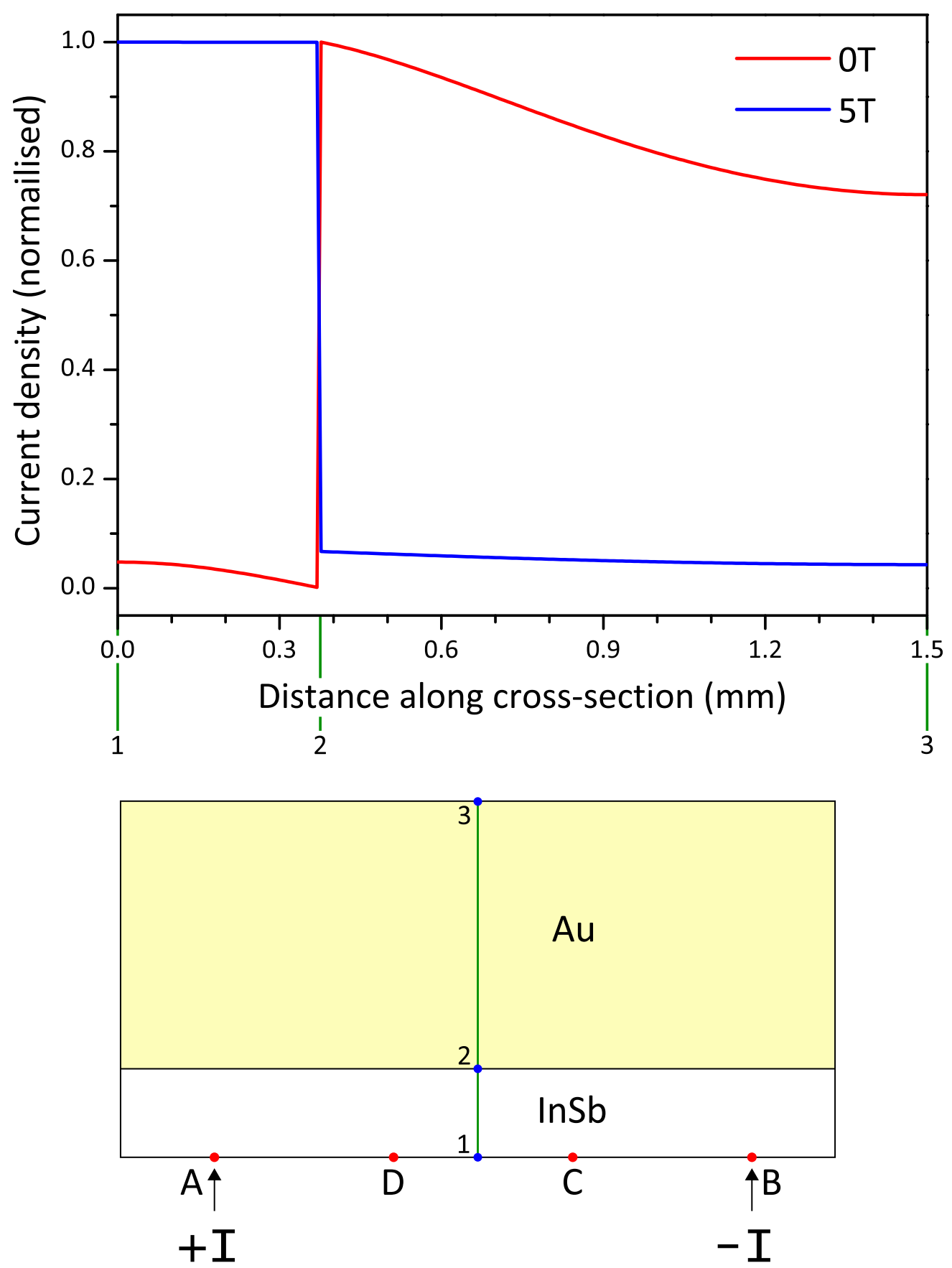

FIGURE 4.17: A cross-section showing the variation in current density (normalised) along the vertical line throughout the system for a linear geometry EMR device with filling factor $\alpha=0.866$ (as seen in Figure 4.14). The points 1 to 3 on the cross-section correspond to points 1 to 3 on the $\mathrm{x}$ axis of the plot. 
In this section, it has been shown that the EMR effect can occur in EMR devices of a linear (externally shunted) geometry. The mechanism for the EMR effect arises in a similar manner as in circular device geometries. However, when comparing a linear device geometry (as in Figure 4.14) to a circular device geometry (as in Figure 3.11) the magnetoresistance results cannot be expected to reach the same magnitude. In the linear device geometry the magnitude of the magnetoresistance is reduced compared to the circular device geometry as the system resistance at high magnetic fields is reduced by the current flow that is forced into the metallic at the semiconductor-metal interface at the left hand side of the device. Practically, a linear device geometry means the fabrication of sensors can be implemented with existing nano-electronic technology therefore reducing the cost of such devices $\frac{14}{14}$. 


\section{Chapter 5}

\section{Influence of Material Parameters}

It has already been established that the EMR effect is a geometric effect. The shape of the system and placement of the contacts has a larger impact on the magnetoresistance than the physical ordinary magnetoresistance of the two components. However, the material parameters of EMR devices are still an important aspect of their design. This section reviews previous research based upon the variation of material parameters in EMR devices. Furthermore, the effect of three specific parameters on the magnetoresistance are investigated: the conductivity ratio; the mobility of the semiconducting region; and the introduction of an interface resistance 16 [17]19 21/86/97/98.

\subsection{Previous Investigations of Material Parameters}

We know the material parameters of EMR devices are an important factor that influence the resultant magnetoresistance. The affect of material conductivity and mobility has been previously investigated theoretically and experimentally in linear EMR devices. Since the EMR mechanism relies upon the switching of current paths from the metallic (low resistance) to semiconducting region (high resistance) upon the application of a magnetic field, we know that a significant difference is required between the conductivities of the metal and semiconductor. In the systems of Solin et al. the conductivity of the metal is 2,430 times that of the semiconductor, large magnetoresistance effects are also reported for systems where the difference varies an order of magnitude either side of this value $^{10[12}$. In order to quantify the effect of this parameter on the magnetoresistance it has been investigated in section 5.2 .

The influence of the material conductivities on the magnetoresistance of linear EMR systems has been the subject of previous investigations ${ }^{17|19| 20}$. In one such investigation 


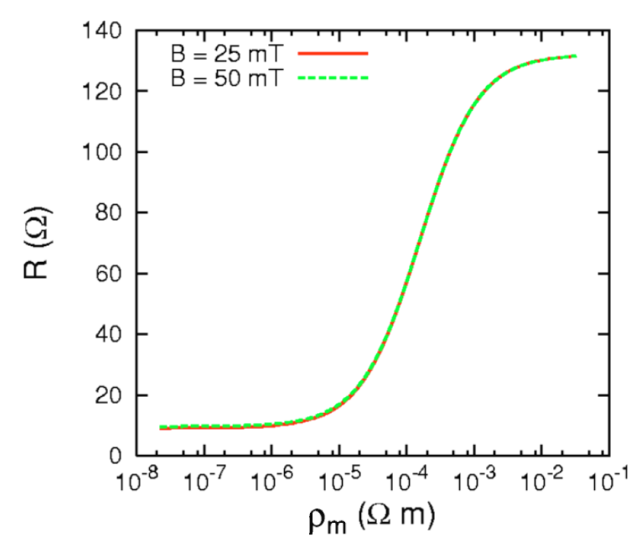

FiguRE 5.1: The influence of the metal resistivity on the resistance of a linear EMR device (FEM model) for two values of magnetic field, $25 \mathrm{mT}$ (red solid line) and $50 \mathrm{mT}$ (green dashed line). (Reproduced from reference [19).

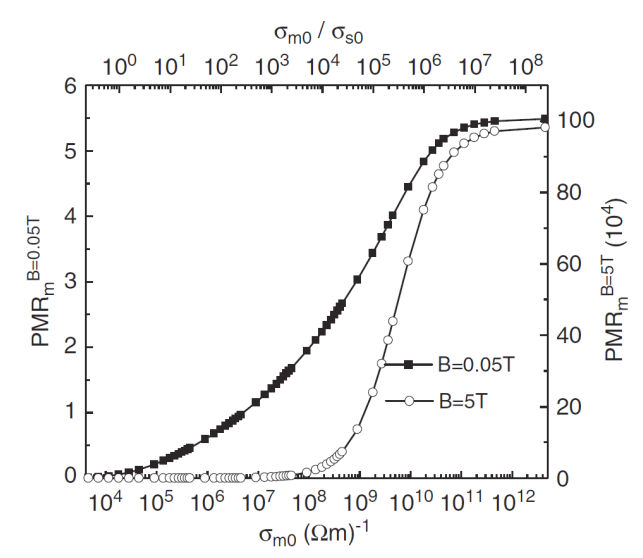

FiguRE 5.2: Magnetoresistance as a function of the conductivity ratio $\left(\sigma_{M} / \sigma_{S}\right)$ for a linear EMR device (FEM model with $\alpha=0.91$ ) for two values of magnetic field, $0.05 \mathrm{~T}$ (filled squares) and 5T (open circles). (Reproduced from reference 20).

the resistivity of the semiconducting region was fixed $\left(\rho_{S}=7 \times 10^{-5} \Omega \mathrm{m}\right)$ and only the resistivity of the metallic region varied. Figure 5.1 shows a plot of system resistance as a function of resistivity of the metallic component for such a linear EMR system 19 . We see that in general an increase in the resistivity of the metal above $2 \times 10^{-6} \Omega \mathrm{m}$ causes the system resistance to rapidly increase. A metal resistivity of below $10^{-6} \Omega \mathrm{m}$ (metal conductivity of $10^{6}$ or greater) does not reduce the system resistance any further. Similarly, an increase in metal resistivity above $0.01 \Omega \mathrm{m}$ does not further increase the system resistance, at this point the metallic region no longer acts as a shunt and the main current flow is directed through the semiconducting region. It was concluded that the EMR effect does not dramatically deteriorate when a metal with a higher resistivity (potentially 100 times larger than $\mathrm{Au}$ ) is used in the construction of an EMR device ${ }^{17 / 19}$.

In such devices it is useful to define the conductivity ratio: $\sigma_{M} / \sigma_{S}$. The subscripts $\mathrm{M}$ and $\mathrm{S}$ correspond to metallic and semiconducting regions respectively. This relative difference between the conductivities of the two materials is the important parameter, not their absolute values. In Figure 5.2 the magnetoresistance of a linear EMR device is presented as a function of conductivity ratio for two values of magnetic field 20 . Generally, we see that larger magnetoresistance values are observed at larger values of conductivity ratio, at both magnetic fields. The increase in magnetoresistance occurs in a much narrower range of conductivity ratio at high field compared to low field. A conductivity ratio of $10^{4}$ is required for a significant magnetoresistance to be achieved ${ }^{20}$. Consequently, a less pure metal could be used in the production of EMR devices and still yield a large magnetoresistance effect. When the conductivity ratio reaches a value of $10^{7}$ or above, the magnetoresistance is not increased further as it has saturated. For this system to 
reach its maximum magnetoresistance the conductivity ratio is required to reach $10^{7}$. For the systems of Solin et al. the conductivity ratio was much lower than this value at 2,430 .

Another material parameter that has a strong influence on the magnetoresistance of an EMR device is the mobility of the semiconducting region. From the experimental results of Solin et al. an expression describing the magnetoresistance at low fields for an EMR system was introduced as follows ${ }^{9}$.

$$
\operatorname{EMR}(B)=g(B)[\mu B]^{2}
$$

Here, $g(B)$ is a geometric factor associated with the system, $\mu$ is the carrier mobility of the semiconducting material and B is the applied magnetic field. This shows the mobility of the semiconducting material is an important parameter in EMR systems. This is also apparent from the experimental EMR devices that have differing material parameters. For circular EMR systems, the largest EMR effect was found to occur in systems with the largest semiconducting mobility of $45,500 \mathrm{~cm}^{2} / \mathrm{Vs}^{9}$. Systems with lower mobility semiconducting films tend to result in smaller magnetoresistance values $\frac{10[12}{2}$. This trend suggests the need for a high mobility semiconductor but since the experimental systems vary in many other ways this indication cannot be relied upon alone.

The effect of the mobility of the semiconducting region on the magnetoresistance has been studied for linear EMR devices $16|17| 19|20| 97$. The treatment of the mobility has been carried out in two ways in these studies, either: a change in mobility with a fixed value of carrier concentration resulting in different values of conductivity $16|17| 19 \mid 97$; or a change in mobility with a fixed value of conductivity resulting in different carrier concentrations 20 . The difference between these two approaches is significant. When the carrier concentration is fixed a change in the mobility affects both the dimensionless magnetic field $(\beta=\mu B)$ and the zero field conductivity that forms the conductivity tensor. An increase in the dimensionless magnetic field tends to increase the magnetoresistance as the Hall angle is large for a given magnetic field. However, increasing the mobility with a fixed carrier concentration causes an increase in the semiconductor conductivity. This acts to reduce the magnetoresistance effect (as seen in Section 5.2) when there is not a significant difference between the conductivity of the metal and the semiconductor. This means this approach is more complex than when the zero field conductivity is fixed. Fixing the value of zero field conductivity means a change in mobility only influences the dimensionless magnetic field. Figures 5.3 and 5.4 show results for the analysis of the first type (where a change in $\mu$ changes $\sigma$ with $\mathrm{n}$ constant), while Figure 5.5 considers 
a change in mobility of the second approach (where a change in $\mu$ changes $\mathrm{n}$ with $\sigma$ constant).

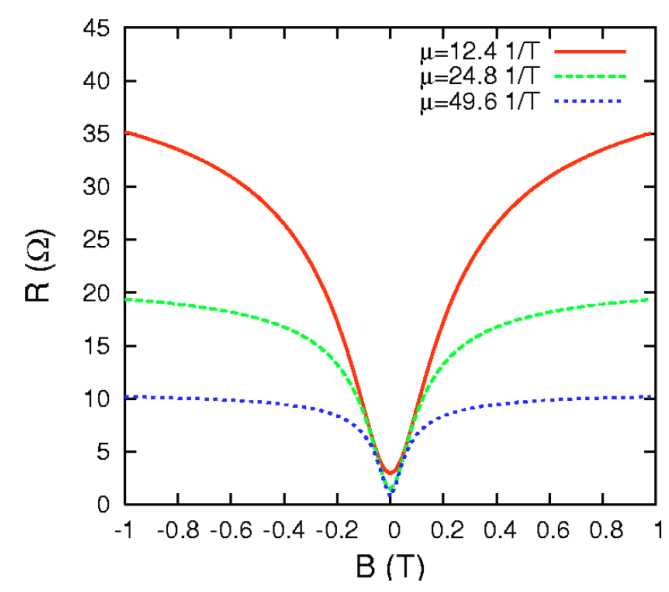

FiguRE 5.3: The resistance as a function of applied magnetic field for three values of semiconductor mobility: $12.4 \mathrm{~m}^{2} / \mathrm{Vs}$ (red solid line); $24.8 \mathrm{~m}^{2} / \mathrm{Vs}$ (green dashed line); and $49.6 \mathrm{~m}^{2} / \mathrm{Vs}$ (blue dashed line). These results were created from a FEM model with a constant carrier density for a linear geometry EMR device.

(Reproduced from reference [19).

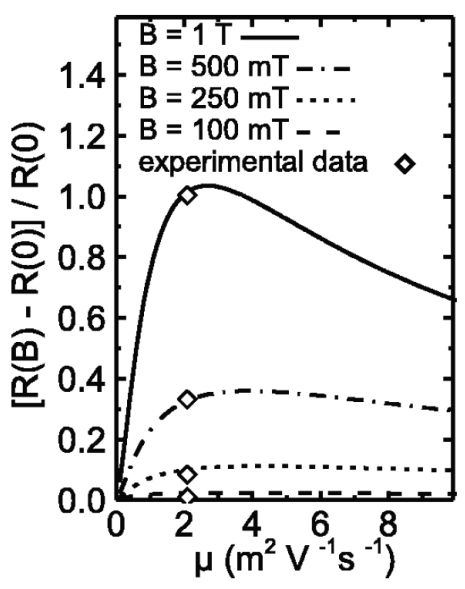

Figure 5.4: Magnetoresistance as a function of semiconductor mobility at four values of magnetic field $(100 \mathrm{mT}, 250 \mathrm{mT}, 500 \mathrm{mT}$ and $1 \mathrm{~T})$ showing the comparison to experimental data. These results were created from a FEM model with a constant carrier density for an EMR device of a linear geometry. (Reproduced from reference 16).

From Figure 5.3 the resistance of a linear EMR device is plotted as a function of magnetic field for three values of semiconductor mobility. We see that in general the resistance difference between the zero field value and the value at $1 \mathrm{~T}$ decreases with an increase in semiconductor mobility. However, the zero field resistance of the system appears to decrease with an increase in mobility. Since the magnetoresistance is defined as the change in resistance divided by the zero field value, it is not immediately apparent how the magnetoresistance would be affected by mobility in this case.

Figure 5.4 shows the change in magnetoresistance with semiconductor mobility for four values of magnetic field, for a similar linear EMR device as in Figure 5.3 . Here, we see an increase in magnetoresistance at low values of mobility, then a reduction in magnetoresistance at large mobilities. This behaviour can be explained in terms of the competition between an increase in dimensionless magnetic field (acting to increase the magnetoresistance) and a decrease in the conductivity ratio (acting to decrease the magnetoresistance). At low values of mobility an increase causes a larger Hall angle at a given magnetic field, thus increasing the magnetoresistance, with the conductivity of the semiconductor sufficiently lower than that of the metal. Therefore, we see the magnetoresistance increase with mobility until it reaches a peak at a mobility of approximately $3 \mathrm{~m}^{2} / \mathrm{Vs}$. For values of mobility above this, the conductivity of the 


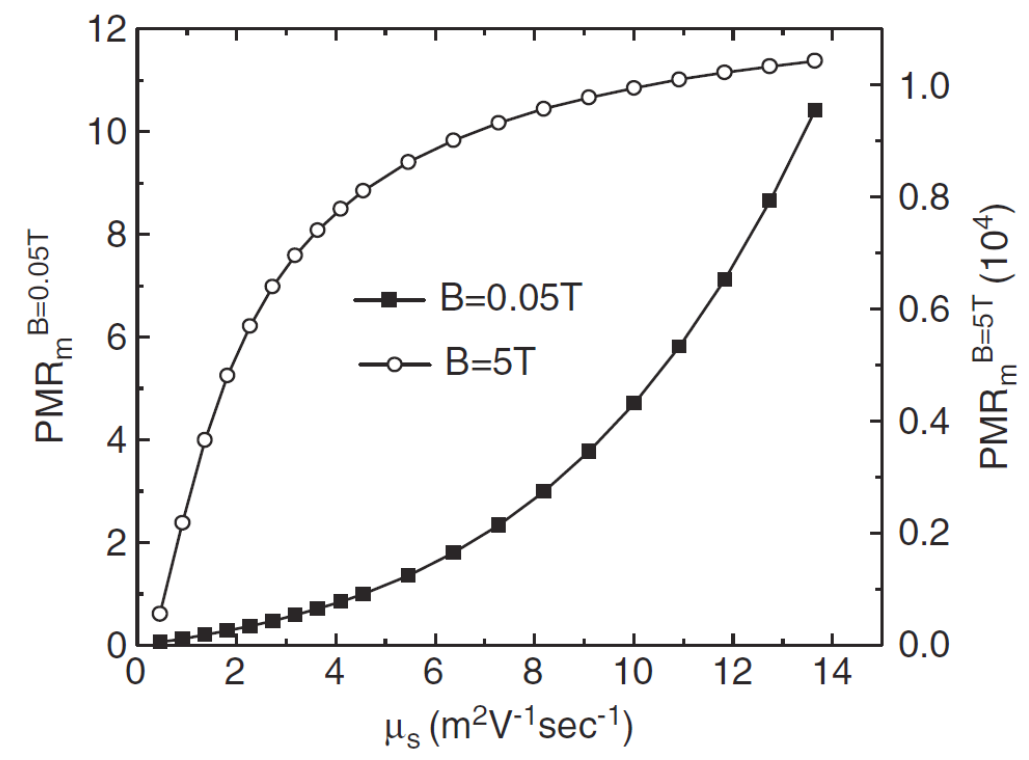

FIGURE 5.5: Magnetoresistance as a function of semiconductor mobility for two values of magnetic field, $0.05 \mathrm{~T}$ (filled squares) and $5 \mathrm{~T}$ (open circles) for a FEM model of a linear EMR system. We see a quadratic dependence of the magnetoresistance with mobility at low field and signs of saturation for large mobilities at high fields. (Reproduced from reference 20 ).

semiconductor has changed sufficiently to reduce the conductivity ratio and thus cause a reduction in the magnetoresistance. Due to this peak in the magnetoresistance it was suggested that for future EMR devices the mobility could be tuned to enhance the magnetoresistance effect.

Figure 5.5 shows the magnetoresistance of a linear EMR device as a function of semiconductor mobility at two magnetic fields, low field (0.05T) and high field (5T). Here, the results were created using a FEM model whereby the zero field conductivity was kept constant with a change in mobility, with the variability accounted for by different carrier concentrations 20 . Therefore, a change in mobility affects the dimensionless magnetic field only. The dependence of the magnetoresistance on the magnetic field at low fields in Figure 5.5 shows a quadratic behaviour which is consistent with Equation 5.1 . At high field we see a reduced rate of increase in magnetoresistance with semiconductor mobility suggesting saturation occurring for large mobilities. At large mobilities the Hall angle is enhanced for a given value of magnetic field. Therefore, the switching of current between the metallic and semiconducting regions is more effective. As a result of this behaviour it was noted that to achieve large magnetoresistance values, high mobility semiconductors are required in the system construction. In the same work, the effect of the mobility of the metallic region on the magnetoresistance was presented. It was found the mobility of the metal did not greatly influence the magnetoresistance over a large range of values. However, the magnetoresistance was reduced when the mobility of the metallic region was above $100 \mathrm{~m}^{2} / \mathrm{Vs}^{20}$. 


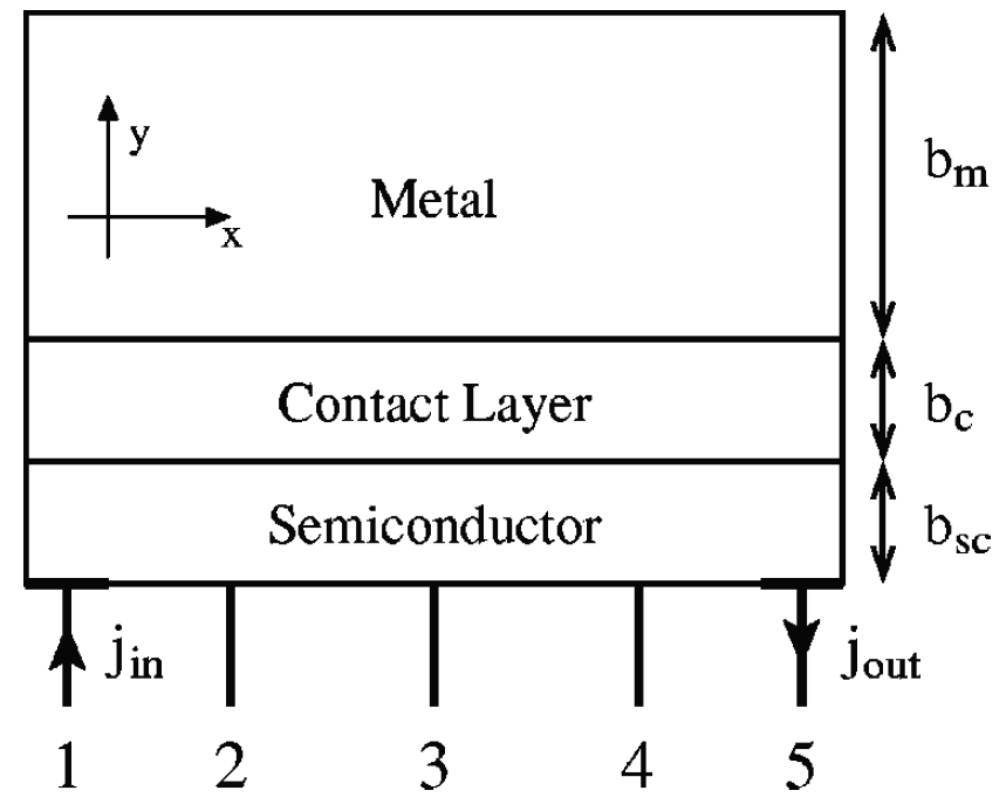

FIGURE 5.6: The introduction of an intermediate layer in order to model the contact resistance at the semiconductor-metal interface in a linear EMR system. (Reproduced from reference 19).

At the interface between the semiconducting and metallic regions in an experimental EMR system, the current flow undergoes some resistance. This may be due to the quality of the contact as a result of fabrication techniques or to the formation of a Schottky barrier. Since basic FEM models assume an ideal ohmic contact at the interface, more realistic results can be obtained when the semiconductor-metal interface is considered. The interface resistance of linear EMR devices has previously been investigated 1 [17/19/21/86/97/98 . Figure 5.7 shows the variation of system resistance with magnetic field, for linear EMR devices with varying values of contact resistivity. Here, the contact resistance is modelled by the introduction of an intermediate layer between the semiconductor and the metal (see Figure 5.6). The conductivity tensor in the intermediate region took the following form:

$$
\hat{\sigma}=\left(\begin{array}{ll}
0 & 0 \\
0 & \sigma_{c}
\end{array}\right)
$$

This provides two purposes: firstly, the current flow was directed along the y axis across the interface; and secondly, the magnetic field did not influence the value of the contact resistance. From Figure 5.7 we see that for the largest value of contact resistivity the system resistance is high for all magnetic fields. This is because even at low fields the high interface resistance prohibits current from entering the metallic region. In general the zero field resistance increases with contact resistivity, as expected. At high fields the resistance 


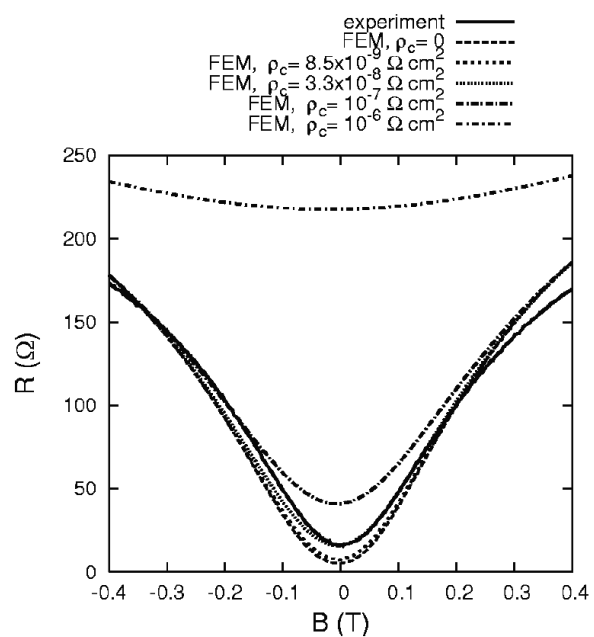

Figure 5.7: Resistance of a linear EMR device as a function of magnetic field for various values of contact resistance, compared to experimental data. The largest value of contact resistance dramatically increases the system resistance at all magnetic fields. (Reproduced from reference 19).

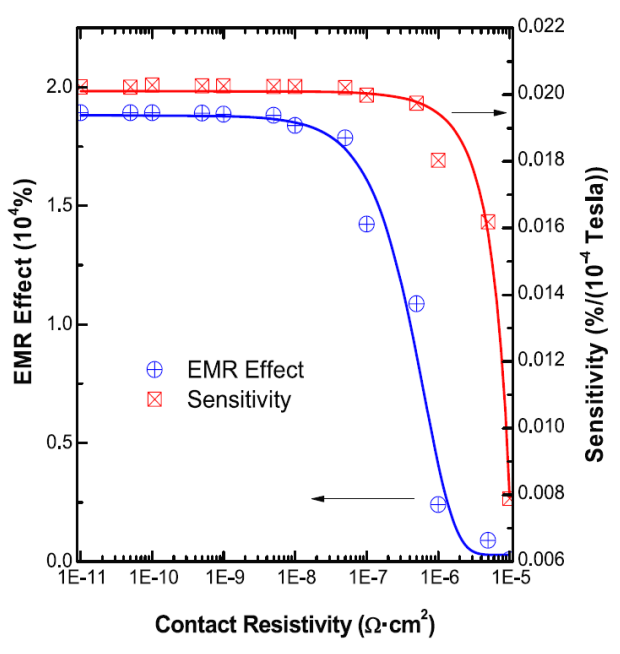

Figure 5.8: Magnetoresistance as a function of contact resistivity (shown in blue) for a FEM model of a linear geometry EMR device, at an applied magnetic field of $1 \mathrm{~T}$. We see that large values of contact resistivity dramatically reduce the EMR effect. (Reproduced from reference

21).

tends to be largely unaffected by the introduced contact resistivity. This is because the majority of the current flow at higher fields is directed through the semiconducting region, and therefore does not cross the semiconductor-metal interface. In Figure 5.7 the contact resistivity was used as a parameter in order to fit the results of the FEM model to experimental data. The best fit between the model and the experimental data was found for a contact resistivity of $3.3 \times 10^{-8} \Omega \mathrm{cm}^{2}$. It was noted that this value was an overestimate of the contact resistivity, since all variability between the model and the experimental system was accounted for solely by the introduction of a contact resistance.

Figure 5.8 shows a similar approach to modelling the interface resistance for a linear EMR device. Here, the magnetoresistance is plotted (seen in blue) as a function of contact resistivity over a range from $10^{-11}$ to $10^{-5} \Omega \mathrm{cm}^{2}$. The EMR effect drops off exponentially for a value of contact resistivity of $10^{-8} \Omega \mathrm{cm}^{2}$ or larger 21 . This result is consistent with that in Figure 5.7, and suggests that for a large EMR effect to occur in linear EMR devices the contact resistivity must be below a critical value, above which the EMR effect vanishes. Here, the units of $\rho_{c}$ are $\Omega \mathrm{cm}^{2}$ since the width $\left(b_{c}\right)$ and conductivity $\left(\sigma_{c}\right)$ of the contact layer and the thickness of the system were combined to produce a specific contact resistance that could be directly compared to an experimental result $\frac{19}{}$. 


\subsection{Influence of Conductivity}

In order to investigate the effect of varying the zero field conductivity of both the semiconducting and metallic regions on the magnetoresistance, we have seen the conductivity ratio is a useful parameter, $\sigma_{M} / \sigma_{S}$. For a circular geometry EMR system with filling factor of 8/16 (identical to those seen in Chapter 3 ) the magnetoresistance was measured for various values of conductivity ratio, with the charge carrier mobilities fixed to those given in Table 3.1. The magnetoresistance as a function of magnetic field (0T to 5T), for various values of conductivity ratio, for a circular EMR system with filling factor of $8 / 16$ is presented in Figure 5.9. Here, a conductivity ratio of 2,430 corresponds to that found in the systems of Solin et al. (material parameters given in Table 3.1).

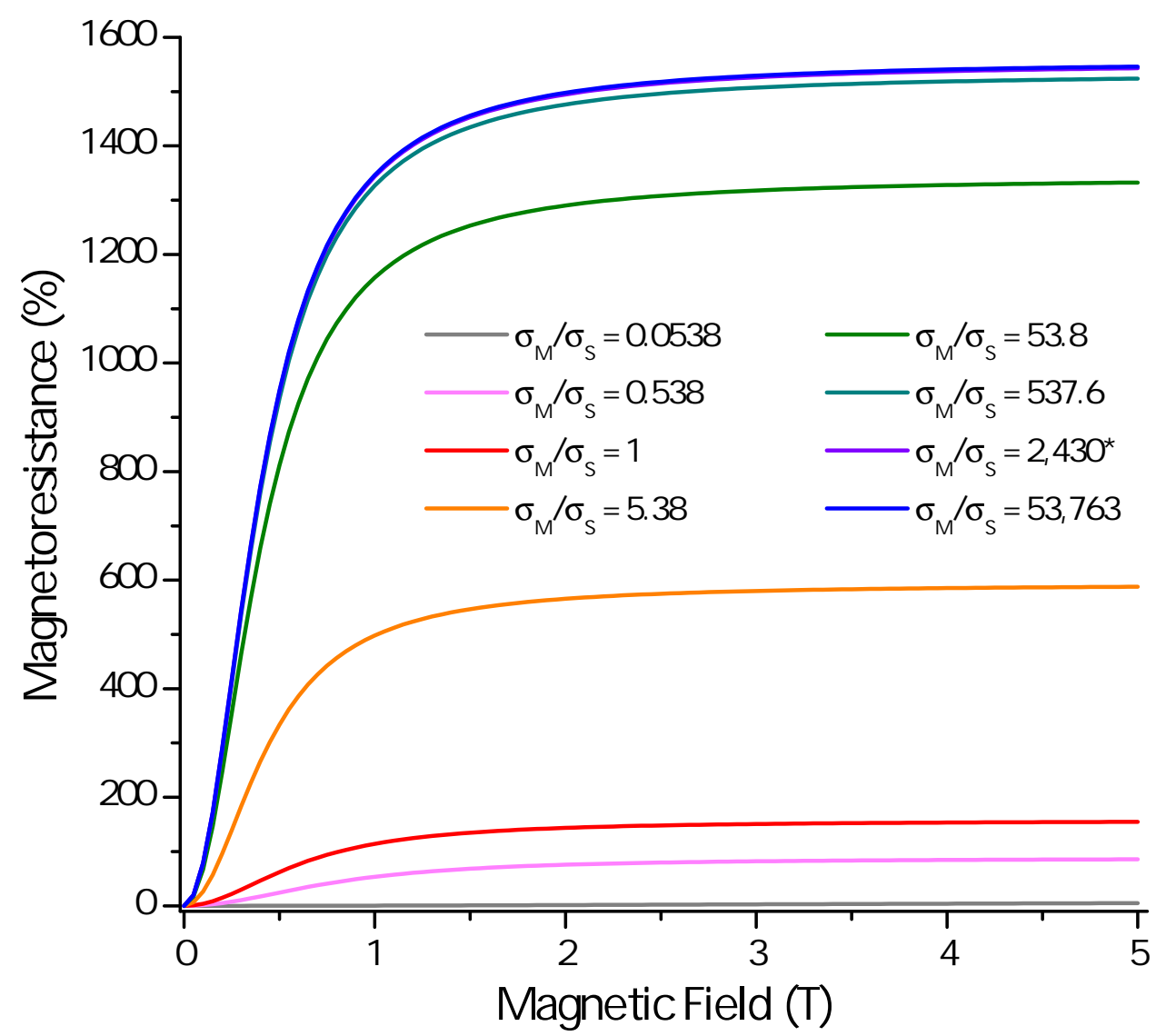

Figure 5.9: Magnetoresistance as a function of applied magnetic field for various values of conductivity ratio $\left(\sigma_{M} / \sigma_{S}\right.$, see legend) for a modified van der Pauw disk with filling factor of $8 / 16$. We see that the greatest magnetoresistance values arise for large values of conductivity ratio (when the conductivity of the metal is significantly greater than that of the semiconductor). The magnetoresistance for the two largest values of conductivity ratio are almost indistinguishable from one another on this plot.

In general, we see the highest values of magnetoresistance are achieved for the largest values of conductivity ratio. Conductivity ratio values of 537.6 or above result in very similar magnetoresistance values where a maximum has been reached. Therefore, any 
further increase in conductivity ratio would not increase the magnetoresistance above this value. The conductivity ratio appears to have a strong effect on the magnetoresistance when its value is 53.8 or below. For small values of conductivity ratio ( 1 or less) the metallic region no longer acts as a short circuit and the EMR effect vanishes. This is consistent with the EMR mechanism where the majority of the current would flow through the semiconducting region for all values of magnetic field. Figure 5.9 highlights the need for the metallic region to have a significantly higher conductivity than the semiconducting region.

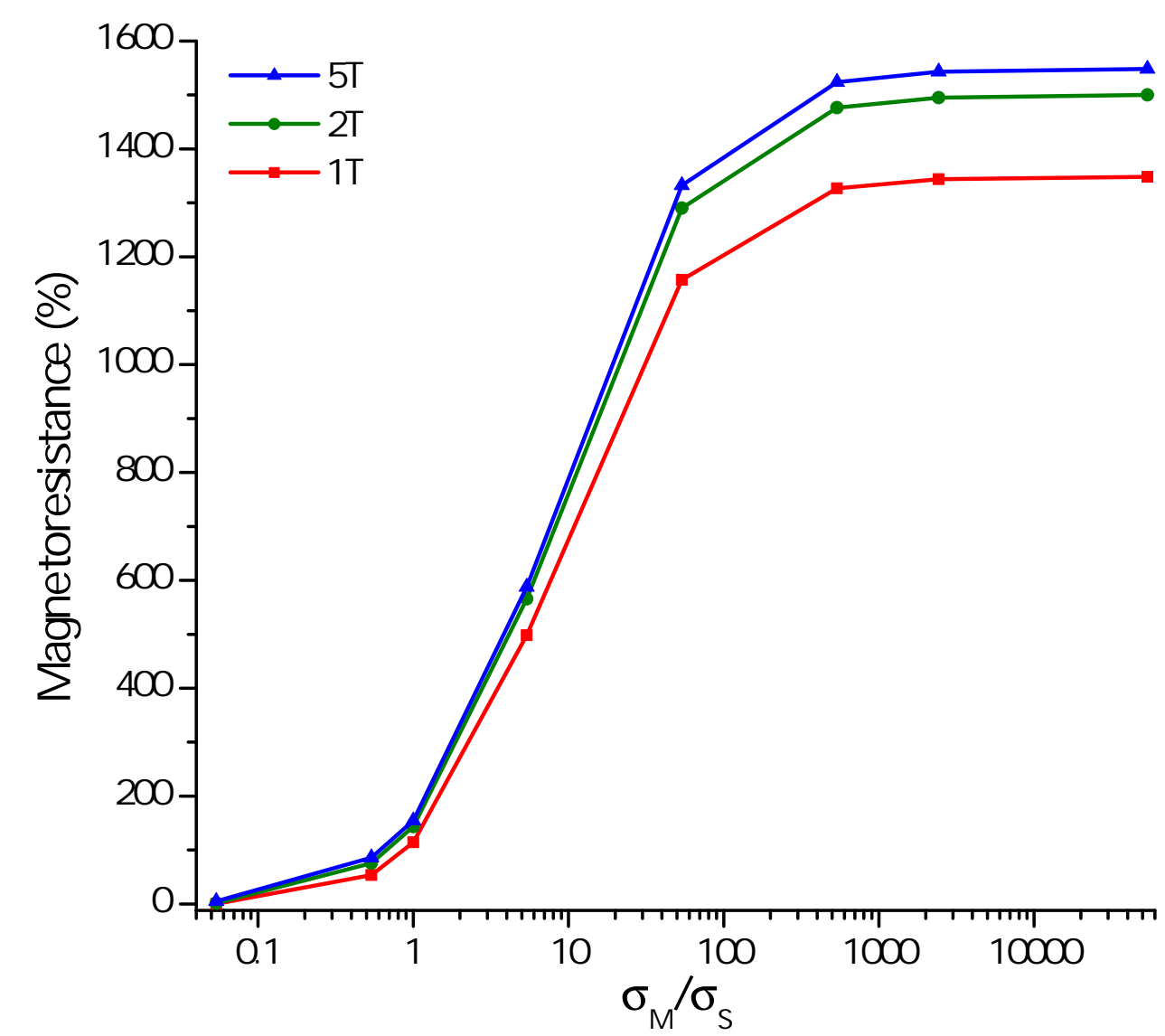

Figure 5.10: Magnetoresistance against conductivity ratio $\left(\sigma_{M} / \sigma_{S}\right)$ for three values of magnetic field: $1 \mathrm{~T}$ (red); $2 \mathrm{~T}$ (green); and $5 \mathrm{~T}$ (blue). These results are for the same system as in Figure 5.9 with filling factor of 8/16. In order for a significant magnetoresistance to arise the conductivity ratio is required to be larger than two orders of magnitude. The systems studied by Solin et al. have a conductivity ratio of 2,430 and are therefore well within this criteria.

In order to observe this in more detail we look at Figure 5.10 where the magnetoresistance has been plotted as a function of conductivity ratio for three values of magnetic field (1T, $2 \mathrm{~T}$ and $5 \mathrm{~T})$. Here, we see the same general trend for all three values of magnetic field. For large values of conductivity ratio (larger than two orders of magnitude) we see the magnetoresistance has reached its maximum value. When the conductivity ratio drops below two orders of magnitude we see the magnetoresistance drops rapidly, 
with it vanishing for small values. This dramatic reduction of the magnetoresistance is significant as it shows a clear threshold which the conductivity ratio is required to be above for the effective performance of any future EMR devices. The conductivity ratio of 2,430 (as in the systems of Solin et al.) is well above the minimum two orders of magnitude and therefore a large EMR effect could be obtained using metals with lower conductivities than Au. Section 5.1 reviewed previous investigations into the effect of the conductivity ratio for linear geometry EMR devices ${ }^{19 \mid 20}$. The results presented here for circular geometry devices are in agreement with previous work which is reassuring since the two device geometries should behave in the same manner due to conformal mapping. However, the threshold above which the conductivity ratio should lie is given as four orders of magnitude (larger than $10^{4}$ ) for linear geometry devices. Since the value reported in this Thesis is significantly lower it suggests the range of potential materials that could be used in the production of an EMR device may be broader than current expectations.

\subsection{Influence of Mobility}

A similar method to that used in Section 5.2 was employed to investigate the effect of the charge carrier mobility of the semiconducting region on the magnetoresistance of the system. The conductivity and mobility of the metallic region and the conductivity of the semiconducting region were fixed to the values given in Table 3.1. The mobility of the semiconducting region was then varied (thus producing different values of $\mathrm{n}$ for different values of $\mu$ with $\sigma$ constant). The magnetoresistance, as a function of magnetic field, can be seen in Figure 5.11 for various values of semiconductor mobility for a circular EMR system with filling factor of $8 / 16$. The mobility of the semiconductor varies from 500 to $200,000 \mathrm{~cm}^{2} / \mathrm{Vs}$; including the value of the experimental system of Solin et al. of $45,500 \mathrm{~cm}^{2} / \mathrm{Vs}$.

Saturation of an EMR device occurs when the majority of the current has been expelled from the metallic region and is forced to flow through the semiconducting material upon the application of a magnetic field. This occurs when the Hall angle $\left(\tan \theta_{\mathrm{H}}=\mu B_{z}\right)$ approaches $90^{\circ}$. Therefore, we would expect that a larger semiconductor mobility would produce a larger Hall angle at a given magnetic field, resulting in a greater proportion of the current being expelled from the metallic region, causing saturation to occur at a lower magnetic field. The observations presented in this Thesis (seen in Figure 5.11) are consistent with this mechanism. In general a higher semiconductor mobility results in a larger magnetoresistance value at a given magnetic field. The three largest values of semiconductor mobility $\left(\mu_{S}>20,000 \mathrm{~cm}^{2} / \mathrm{Vs}\right)$ show saturation of the magnetoresistance 


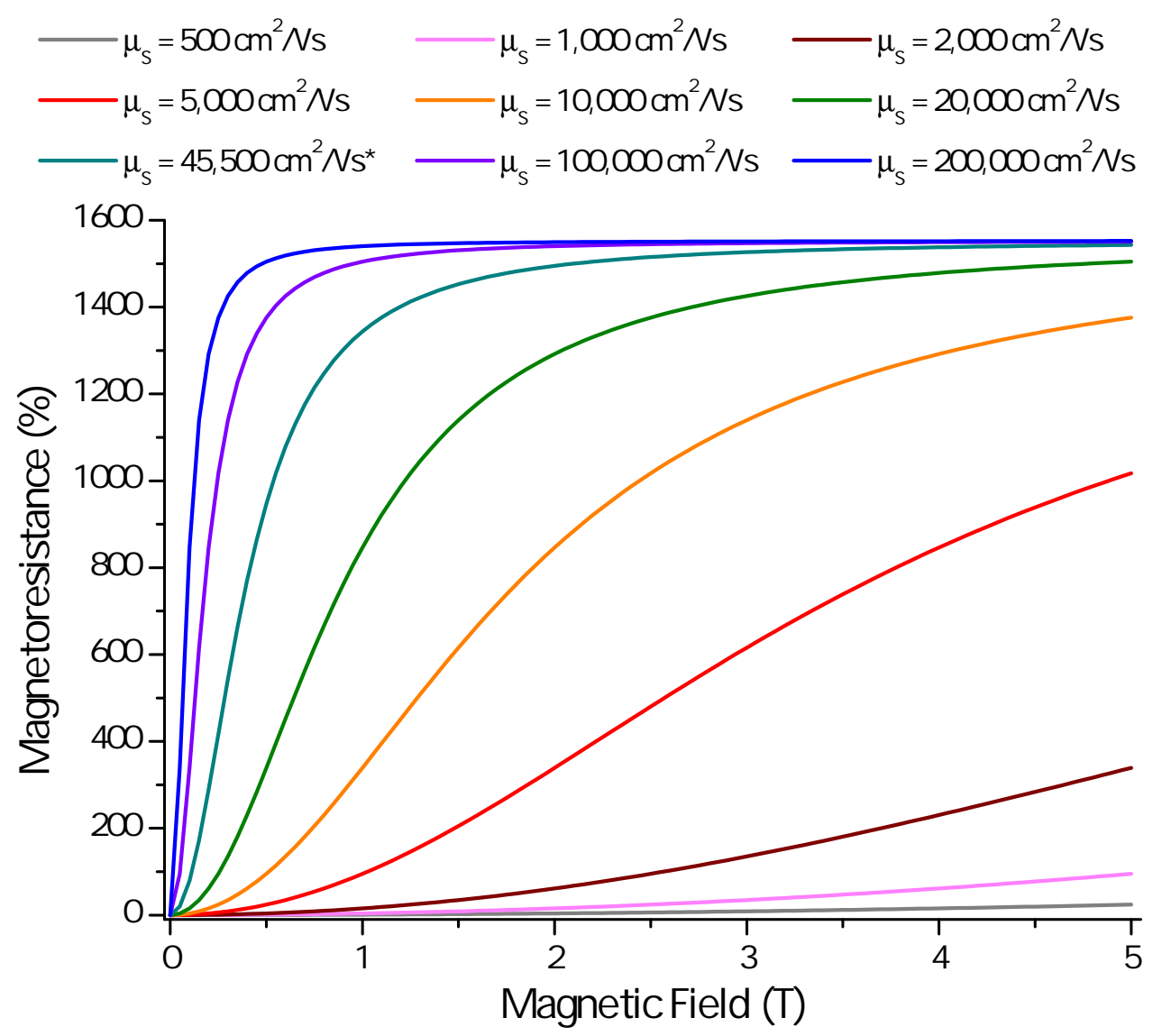

FIgURE 5.11: Magnetoresistance as a function of applied magnetic field for various values of semiconductor mobility for a modified van der Pauw disk with filling factor of $8 / 16$. Here, the mobility of the metallic region was fixed at $\mu_{M}=50 \mathrm{~cm}^{2} /$ Vs (the same as that used in Solin et al. experiments). We see that larger values of semiconductor mobility result in the magnetoresistance saturating at a smaller magnetic field and thus producing larger magnetoresistance values at a given field (below the saturation point).

The semiconductor mobility in the systems of Solin et al. was: $\mu_{S}=45,500 \mathrm{~cm}^{2} / \mathrm{Vs}$.

at $5 \mathrm{~T}$. Here, these three magnetoresistance values converge to the maximum achievable value (approximately 1,500\%). Increasing the semiconductor mobility acts to reduce the magnetic field at which the magnetoresistance saturates. The magnetoresistance of systems with mobilities of $20,000 \mathrm{~cm}^{2} /$ vs or below have not achieved saturation in a magnetic field of $5 \mathrm{~T}$ and would require increasingly high magnetic fields to do so. Figure 5.12 shows the variation in magnetoresistance with semiconductor mobility for two values of magnetic field $(0.05 \mathrm{~T}$ and $5 \mathrm{~T})$. Here, the lines have been fitted in order to show the trend in each case. At low magnetic field, the magnetoresistance increases quadratically with semiconductor mobility, in agreement with equation 5.1 .

Since practical applications of magnetic field sensors mainly occur in low magnetic fields, future EMR devices must be produced from high mobility semiconductors, in order to achieve the large EMR values at these fields. Investigations into the effect of the charge carrier mobility of the semiconducting material have been previously reported 


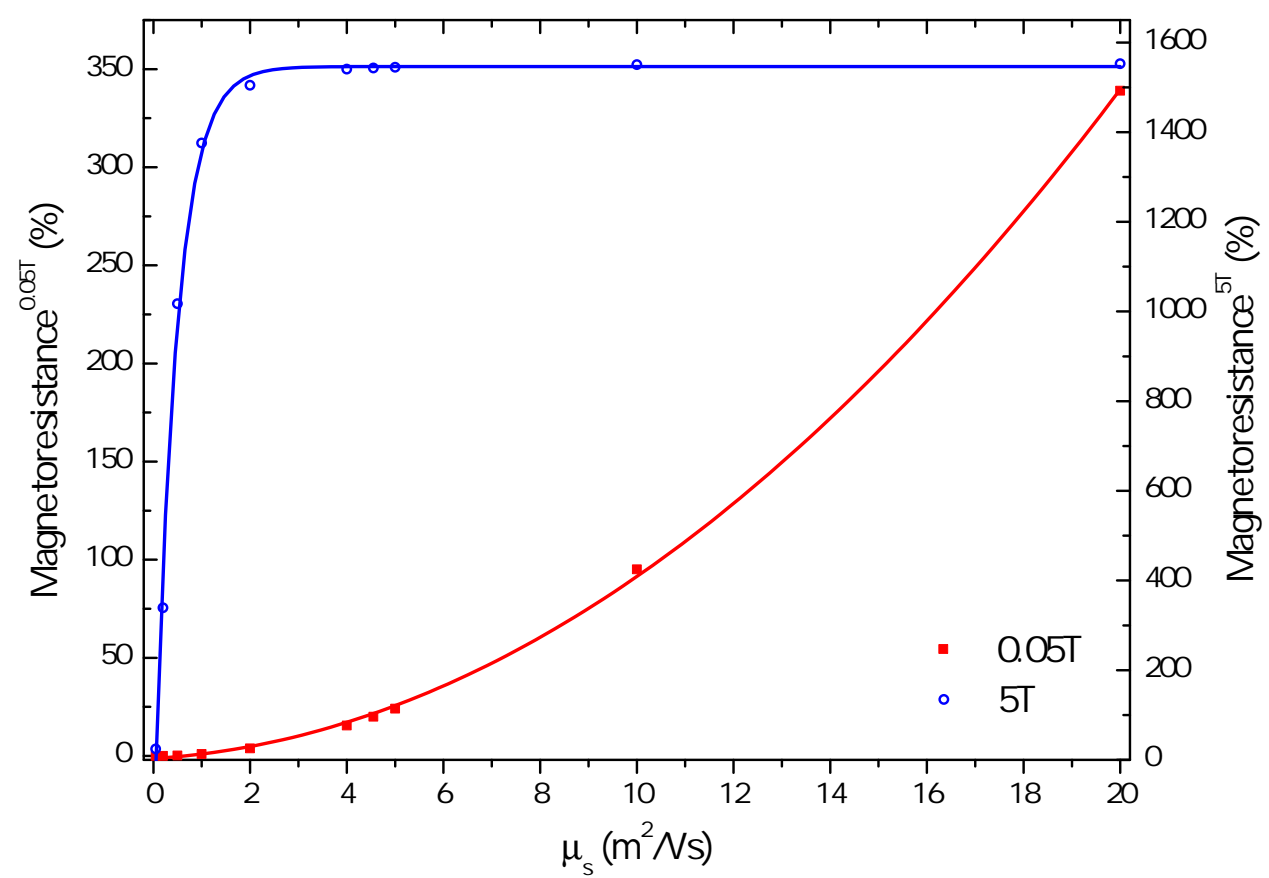

Figure 5.12: Magnetoresistance against semiconductor mobility for two values of magnetic field, $0.05 \mathrm{~T}$ (red filled squares) and $5 \mathrm{~T}$ (blue open circles). The system responsible for these results is the same as in Figure 5.11 with a filling factor of 8/16. The lines have been fitted in order to visualise the trend in each case. At $0.05 \mathrm{~T}$ we see a quadratic dependence of the magnetoresistance with semiconductor mobility.

for linear geometry EMR devices $\frac{16|19| 20}{}$. The work of Holz et al. reports a decrease in magnetoresistance with an increase in semiconductor mobility. However, their analysis differs from that presented in this Thesis. In their analysis the carrier density is kept constant, with a change in mobility causing the zero field conductivity to vary, along with the Hall angle ${ }^{\sqrt{16}}$. The approach described here is consistent with that of Rong et al. where the variation of the semiconductors mobility is absorbed by a change in the carrier density resulting in a constant zero field conductivity 20 . Furthermore, the results presented here are consistent with those found by Rong et al. for linear geometry EMR devices, which is particularly apparent when Figures 5.5 and 5.12 are compared.

Figure 5.13 presents the magnetoresistance against dimensionless magnetic field $(\mu B)$ for a modified van der Pauw disk with filling factor of 8/16 (same data as in Figure 5.11). Here, we see that all nine values of semiconductor mobility investigated in Figure 5.11 are equivalent and are found to overlap producing a single curve. Figure 5.13(a) shows the magnetoresistance increases quadratically with dimensionless magnetic field at low magnetic fields, in agreement with Equation 4.1. The magnetoresistance can be seen to saturate for large magnetic fields in Figure 5.13(b). This result shows that both the magnetic field and the semiconductor mobility are required to be maximised in order for a large EMR effect to be observed. 

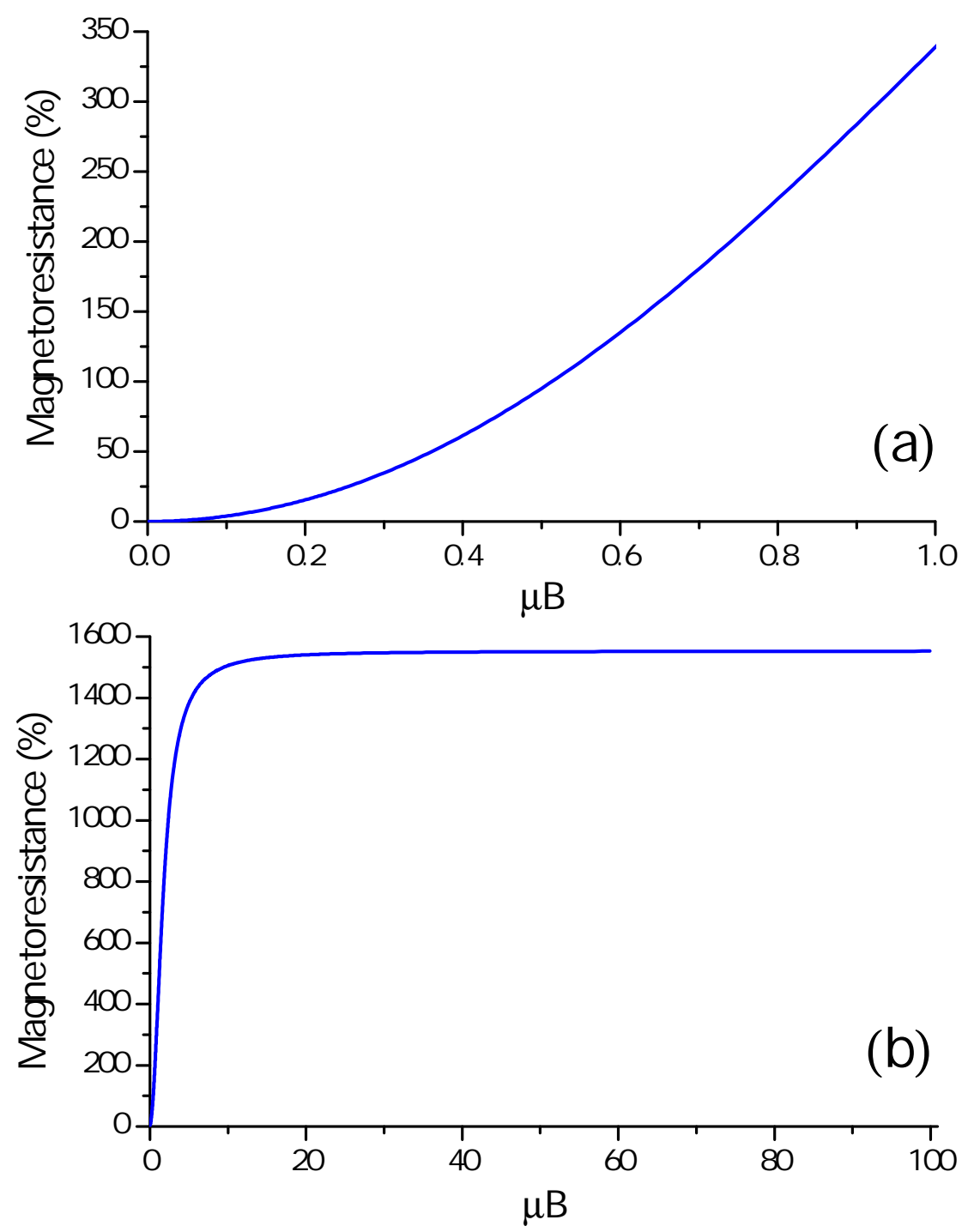

FIgURE 5.13: Magnetoresistance against dimensionless magnetic field $(\mu B)$ for a modified van der Pauw disk with filling factor of 8/16: (a) and (b) present the low and high magnetic field cases. Here, the results for the nine values of semiconductor mobility presented in Figure 5.11 are found to overlap when plotted against the dimensionless magnetic field, resulting in a single curve. In (a), at low values of $\mu B$, we see the magnetoresistance increase quadratically, while saturation of the magnetoresistance can be observed in (b) for high magnetic fields.

\subsection{Influence of Interface Resistance}

The formation of a Schottky barrier at a semiconductor-metal interface results in a reduction of current flow between the two materials, with the interface having an associated contact resistance. In order to model the effect of the formation of a Schottky barrier a contact resistance at the semiconductor-metal interface was introduced with the addition of a thin intermediate layer (see Figure 5.14.)

The thickness of the intermediate layer was set at $1 \%$ of the system radius, with the 


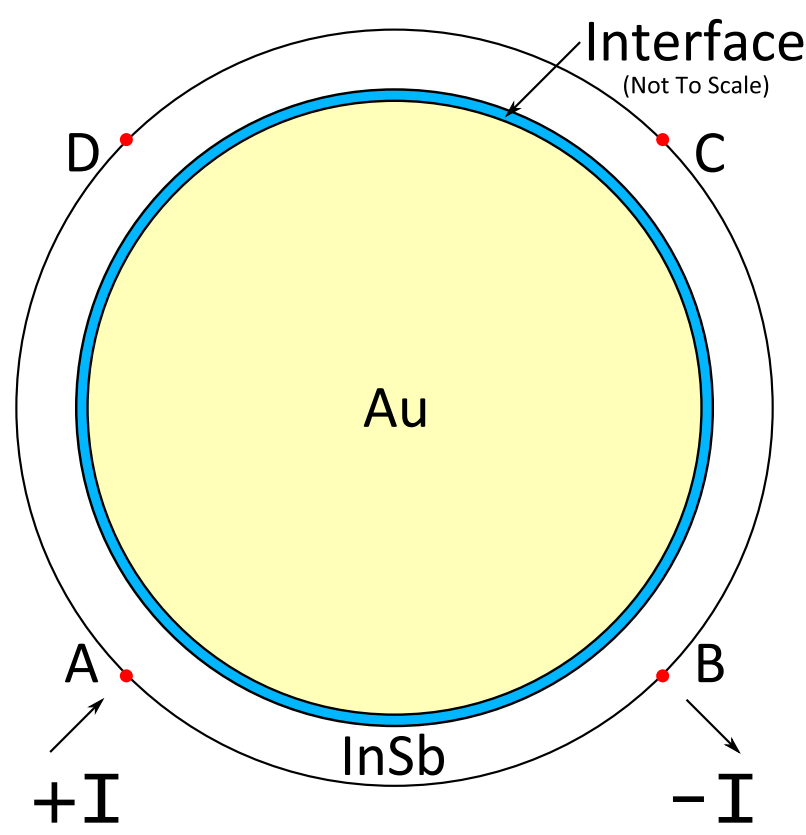

Figure 5.14: The geometry of a modified van der Pauw disk used to model the effect of a contact resistance at the semiconductor-metal interface. The system consists of an outer semiconducting disk ( $1 \mathrm{~mm}$ diameter) with a concentric embedded metallic inclusion (filling factor of 13/16). A thin intermediate layer (thickness of $5 \mu \mathrm{m}$ ) is introduced at the semiconductor-metal interface, with four contacts placed equidistant around the disks perimeter.

radius equal to $r_{a}+\left(r_{b} / 100\right)$. The conductivity tensor in this intermediate region was defined differently to the rest of the system, being of the following form

$$
\hat{\sigma}=\left(\begin{array}{cc}
\sigma_{0} & 0 \\
0 & \sigma_{0}
\end{array}\right) .
$$

Therefore, the interface conductivity is independent of the applied magnetic field. The resistivity tensor is the inverse of the conductivity tensor, therefore the resistivity of the intermediate layer is described by the following expression

$$
\hat{\rho}=\left(\begin{array}{cc}
\rho_{c} & 0 \\
0 & \rho_{c}
\end{array}\right) .
$$

Here, the contact resistivity has units of $\Omega \mathrm{m}$ as the conductivity of the intermediate layer has simply been inverted. The magnetoresistance as a function of magnetic field for various values of contact resistivity $\left(\rho_{c}\right)$ is presented in Figure 5.15 for a circular EMR device with filling factor of 13/16 (see Figure 5.14). The figure also contains the magnetoresistance for a model of the same system without the consideration of a contact resistance for comparison to experimental data. 


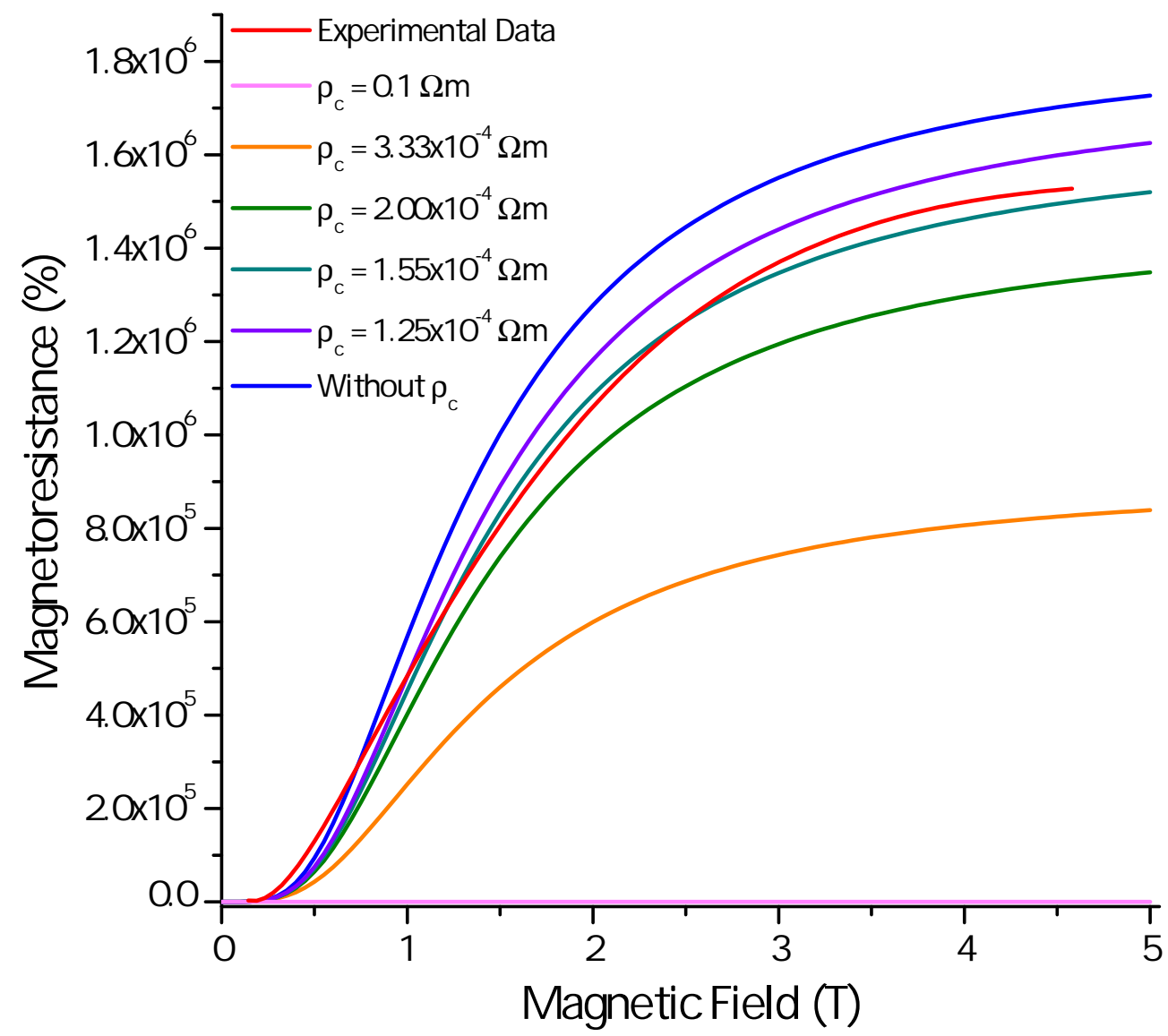

FIGURE 5.15: Magnetoresistance as a function of applied magnetic field for various values of semiconductor-metal contact resistivity. The system responsible for these results (see Figure 5.14 has a filling factor of 13/16 and contains an intermediate contact layer with thickness of $5 \mu \mathrm{m}$. The experimental data (extracted) is shown in red and the corresponding system without the intermediate interface is shown in blue. We see that, consistent with the mechanism for the effect, larger values of contact resistivity reduce the magnetoresistance. We find that an interface resistivity of $1.55 \times 10^{-4} \Omega \mathrm{m}$ was required to fit the model to the experimental data, thus giving an overestimate of the system's interface resistivity.

From Figure 5.14 the largest magnetoresistance value occurs for the system without the interface resistance considered. In general we see that increasing the interface resistivity reduces the magnetoresistance observed, with the EMR effect vanishing altogether in an extreme case with the largest interface resistivity $\left(\rho_{c}=0.1 \Omega \mathrm{m}\right)$. The mechanism for the EMR effect relies on the switching of current from the metallic region to the semiconducting region upon the application of magnetic field (i.e. current crossing the interface). Therefore, it would be natural to expect the interface resistivity to play an important role in the EMR effect. A system with a large resistance to the flow of current at the interface would therefore not be expected to exhibit the EMR effect, as current flow into the metallic region would be restricted at zero field. With a finite interface resistance the EMR effect would be expected to be reduced from the case without an interface resistance, since a lower proportion of the current would contribute to the effect 

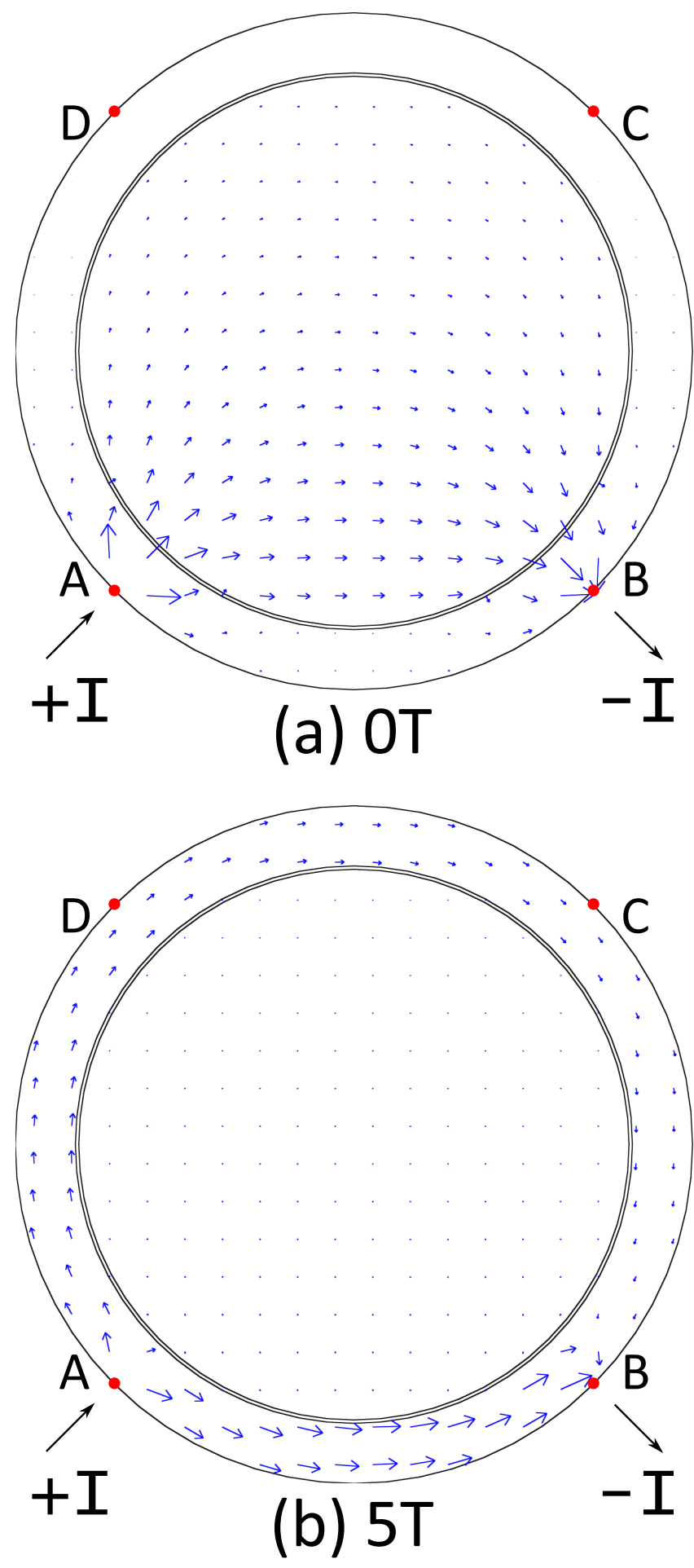

FIGURE 5.16: Here, the current flow is presented for four circular modified van der Pauw disks (all with filling factor of 13/16): (a) and (b) represent a system with contact resistivity of $1.55 \times 10^{-4} \Omega \mathrm{m}$ at magnetic fields of 0 and $5 \mathrm{~T}$ respectively. While (c) and (d) represent a system with contact resistivity of $0.1 \Omega \mathrm{m}$ at magnetic fields of 0 and $5 \mathrm{~T}$ respectively. We see that the EMR mechanism still holds for small values of interface resistance while large values cause the current to flow through the semiconducting disk in both the high (5T) and low (0T) field regimes. This explains the drop in magnetoresistance with increased interface resistance as observed in Figure 5.15 

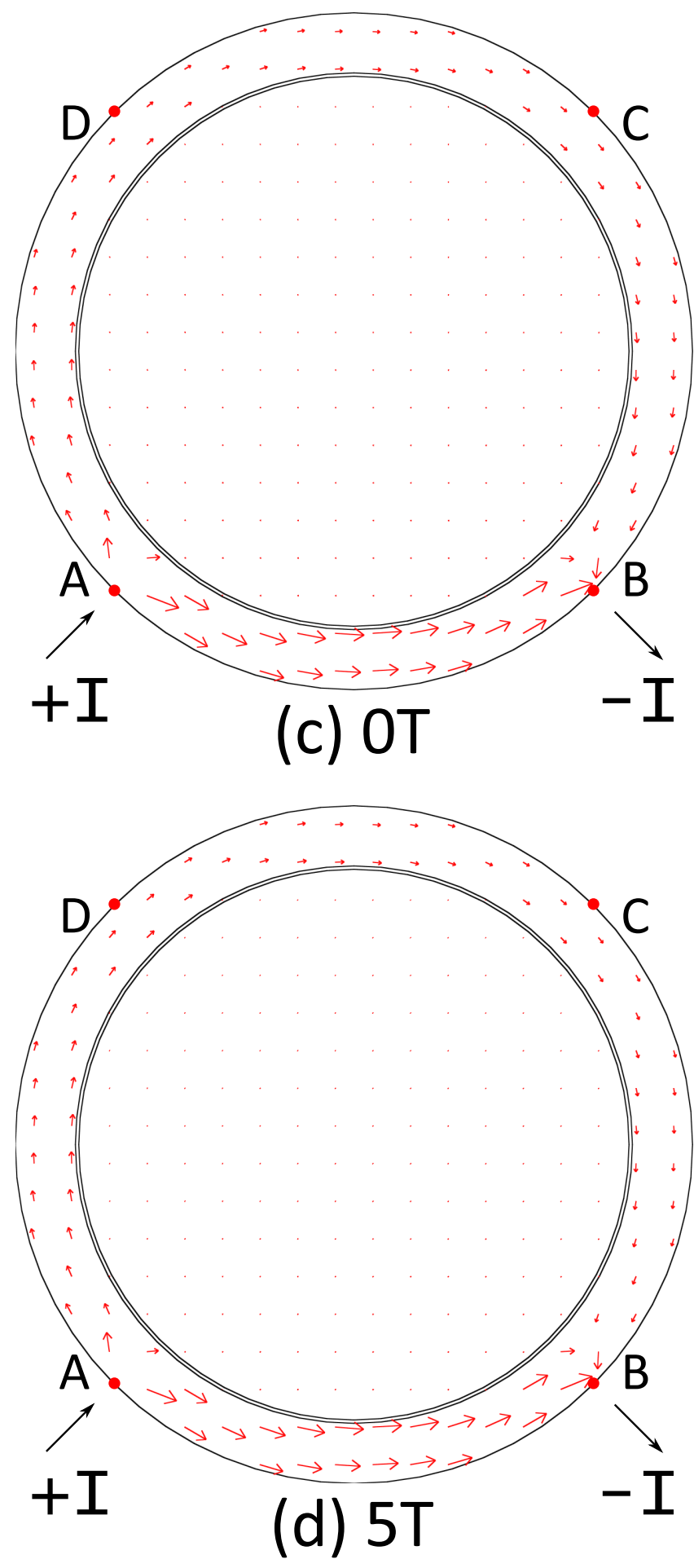

Figure 5.16: (Cont.) Here, the current flow is presented for four circular modified van der Pauw disks (all with filling factor of 13/16): (a) and (b) represent a system with contact resistivity of $1.55 \times 10^{-4} \Omega \mathrm{m}$ at magnetic fields of 0 and $5 \mathrm{~T}$ respectively. While (c) and (d) represent a system with contact resistivity of $0.1 \Omega \mathrm{m}$ at magnetic fields of 0 and $5 \mathrm{~T}$ respectively. We see that the EMR mechanism still holds for small values of interface resistance while large values cause the current to flow through the semiconducting disk in both the high $(5 \mathrm{~T})$ and low (0T) field regimes. This explains the drop in magnetoresistance with increased interface resistance as observed in Figure 5.15 
by entering the metal at zero field. As expected, this is observed in Figure 5.15 .

In order to investigate how the interface resistivity affects the magnetoresistance in the models it is necessary to look at the current flow throughout two systems with different values of contact resistivity $\left(\rho_{c}=1.55 \times 10^{-4} \Omega \mathrm{m}\right.$ for Figures 5.16 (a) and (b) and $\rho_{c}=0.1 \Omega \mathrm{m}$ for Figures $5.16(\mathrm{c})$ and $\left.(\mathrm{d})\right)$ at 0 and $5 \mathrm{~T}$ respectively. This can be seen in Figure 5.16. Here, the arrows represent the direction and relative magnitude of the current flow at various points throughout the systems.

For a system with a reasonably small interface resistivity of $1.55 \times 10^{-4} \Omega \mathrm{m}$, Figures 5.16 (a) and (b) show the expected behaviour of an EMR system where the switching of current paths arise upon the application of a magnetic field. From Figure 5.16(a) we see that at zero magnetic field the majority of the current flows from the semiconducting region, across the interface layer, and into the metallic region of the system. From Figure 5.16(b); with a magnetic field of $5 \mathrm{~T}$, we see the majority of the current flow throughout the system has been expelled from the metal and forced to flow through the semiconducting region. A much larger interface resistivity of $0.1 \Omega \mathrm{m}$ is present in the systems shown in Figures 5.16(c) and (d). This large interface resistivity no longer results in the same behaviour as observed in Figures 5.16(a) and (b). Both Figures 5.16(c) and (d) show an almost identical current flow throughout the systems. The EMR effect vanishes for such a large value of interface resistivity (seen in Figure 5.15) since switching of current paths does not occur, the majority of the current flows through the semiconducting region irrespective of the applied magnetic field in each case. The interface appears to block the flow of current into the metallic region at zero magnetic field.

Since the introduction of an interface resistance reduces the resultant EMR effect, it has been used as a parameter to fit the FEM model to existing experimental data for the same system. The interface resistivity responsible for the best fit to the experimental data being $1.55 \times 10^{-4} \Omega \mathrm{m}$ (see Figure 5.15). It is noted this value is an overestimate of the interface resistivity of the experimental system as all of the variability between the model and the experimental system is accounted for in this parameter, where other factors will also contribute. The effect of the interface resistance on the system resistance of a linear EMR device has previously been reported ${ }^{19|97| 98}$. The low field resistance of these devices was shown to increase with increased interface resistance, resulting from the current flow being directed through the semiconducting region, suppressing the appearance of an EMR effect. The results reported here for EMR devices with a circular geometry are consistent with these findings. For the production of future EMR devices it is important to create a system with a low interface resistance as it is shown to limit the effectiveness of the device. The formation of a Schottky barrier at the semiconductor-metal interface is one such undesirable effect that should be minimised. 


\section{Chapter 6}

\section{Magnetoresistance in Disordered Systems}

This chapter investigates other magnetoresistance effects that have arisen in inhomogeneous semiconductor-metal hybrid systems. This concentrates on the unusually large magnetoresistance effect observed in silver-rich silver chalcogenides. Two inhomogeneous models are then proposed showing a geometric magnetoresistance effect, that increases linearly with respect to the applied magnetic field, can arise in such systems.

\subsection{Magnetoresistance in the Silver Chalcogenides}

\subsubsection{Experimental Data}

The silver chalcogenides are chemical compounds of the form $\mathrm{Ag}_{2} \mathrm{X}$, where $\mathrm{X}$ denotes one of the calcogens, for example $\mathrm{S}$, Se, or Te. At high temperatures ( $\alpha$ phase) these compounds are superionic conductors. However, at lower temperatures ( $\beta$ phase, below approximately 400K) they are non-magnetic n-type semiconductors that exhibit no appreciable magnetoresistance $\mathrm{e}^{22 \mid 99}$. In 1997 silver-rich silver chalcogenides of the form $\mathrm{Ag}_{2+\delta} \mathrm{Se}$ and $\mathrm{Ag}_{2+\delta}$ Te were reported to exhibit a large and unusually linear magnetoresistance that remained even at room temperature ${ }^{22}$. Altering the stoichiometry of the compounds with the addition of a small excess $(\delta=0.01)$ of silver, resulted in a large magnetoresistance effect of up to $200 \%$ in a magnetic field of $5.5 \mathrm{~T}$ at room temperature ${ }^{22}$. The large magnetoresistance effects in the silver-rich silver chalcogenides have been reproduced experimentally, with the linear magnetoresistance observed up to magnetic fields of 55T (as seen in Figure 6.2 23133134100 106. 


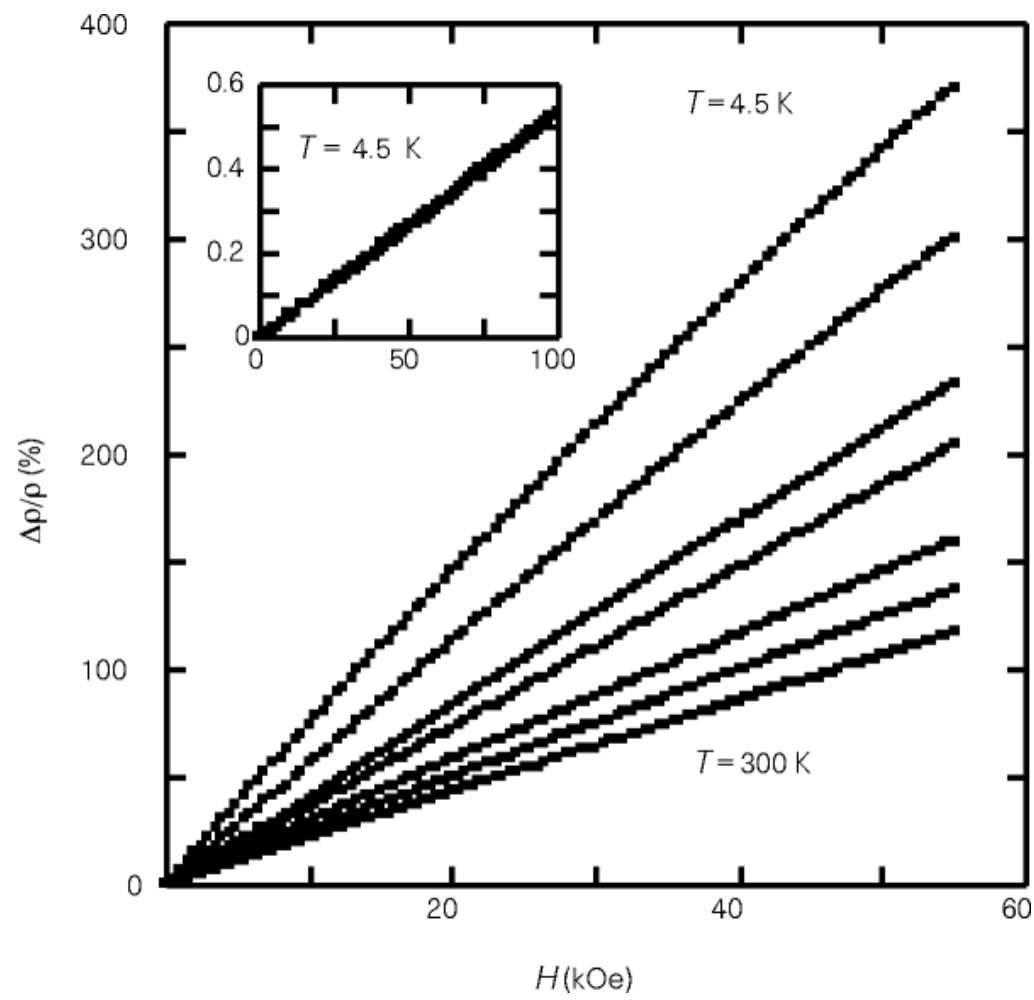

Figure 6.1: Magnetoresistance for $\mathrm{Ag}_{2+\delta} \mathrm{Se}(\delta=0.01)$ as a function of applied magnetic field $(1 \mathrm{~T}=10 \mathrm{kOe})$ for various values of temperature in the range of 4.5 to $300 \mathrm{~K}$. The insert shows the linear field dependence continuing down to fields of a few Oe. (Reproduced from reference 22).

This magnetoresistance effect is unusual since the dependence on magnetic field is linear for a very wide range of magnetic fields, with no sign of saturation even up to very high fields of $55 \mathrm{~T}^{23}$. The magnetoresistance appears to increase linearly with magnetic field for fields larger than 0.001T. Below this value the increase is quadratic. These characteristics make the silver chalcogenides hugely promising candidates for a new range of magnetoresistive devices. These materials are non-magnetic and therefore the signal to noise ratio has the potential to be much larger than devices made from existing technologies (such as GMR that contain ferromagnetic materials). The material parameters of the silver chalcogenide $\mathrm{Ag}_{2+\delta}$ Se are summarised in Table 6.1.

\begin{tabular}{lccc}
\hline & Conductivity & Mobility & Carrier Conc. \\
& $\sigma(\Omega \mathrm{m})^{-1}$ & $\mu\left(\mathrm{cm}^{2} / \mathrm{Vs}\right)$ & $\mathrm{n}\left(\mathrm{m}^{-3}\right)$ \\
\hline $\mathrm{Ag}_{2} \mathrm{Se}$ & $5 \times 10^{4}$ & 2,500 & $1.25 \times 10^{24}$ \\
$\mathrm{Ag}$ & $6.62 \times 10^{7}$ & 70 & $5.91 \times 10^{28}$ \\
\hline
\end{tabular}

TABLE 6.1: Material parameters of the silver chalcogenide materials at zero magnetic field and at room temperature 22

There has been great interest in the microstructure of the silver chalcogenides in recent years due to the large magnetoresistance effect observed 2433/34/104 108. The microstructure 


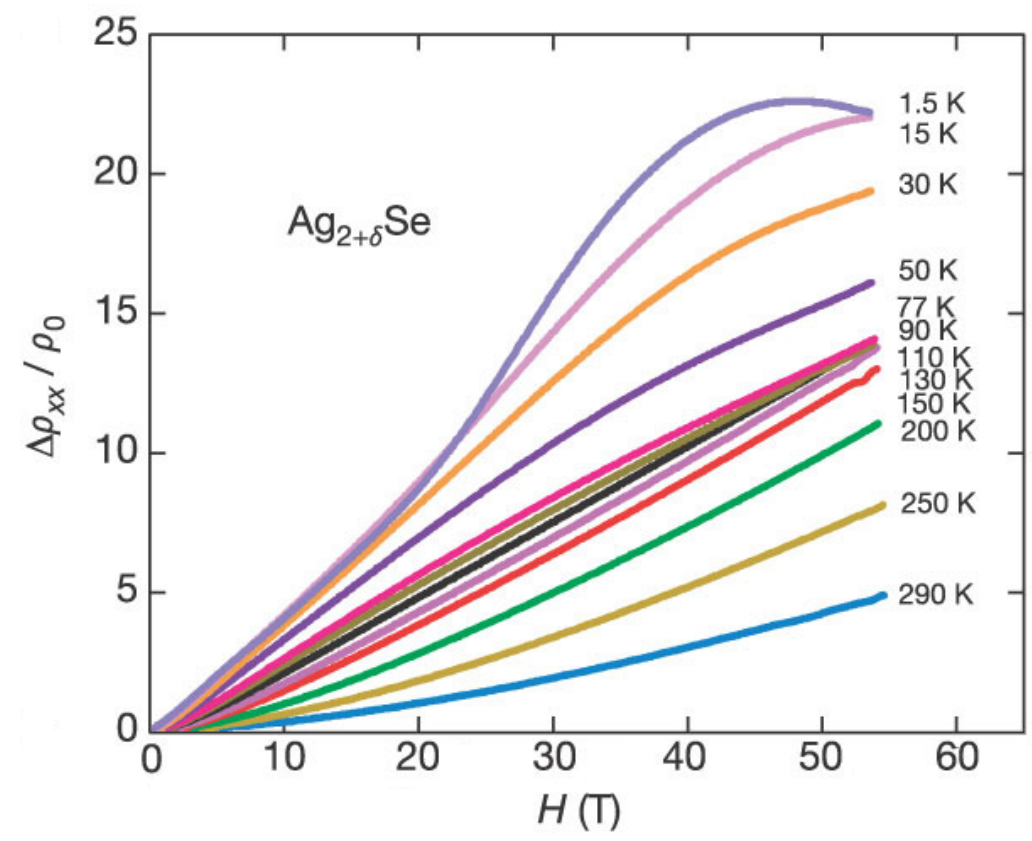

Figure 6.2: Magnetoresistance for $\mathrm{Ag}_{2+\delta} \mathrm{Se}\left(\delta=10^{-4}\right)$ as a function of applied magnetic field (up to $55 \mathrm{~T}$ ) for various values of temperature in the range of $1.5 \mathrm{~K}$ to $290 \mathrm{~K}$.

(Reproduced from reference 23).

of these materials has been shown to have a two-phase character, with the excess silver forming droplets and connecting paths along grain boundaries and at dislocations in the materials. The existence of nanoscale silver inhomogeneities in silver selenide was first reported by Ohachi with subsequent research confirming the formation of such features in the microstructure $2433[34 \mid 104$ 108 . Figure 6.3 shows the formation of silver paths and droplets on the surface of $\mathrm{Ag}_{2} \mathrm{Se}$ with these features primarily located at dislocations or along grain boundaries 24 .

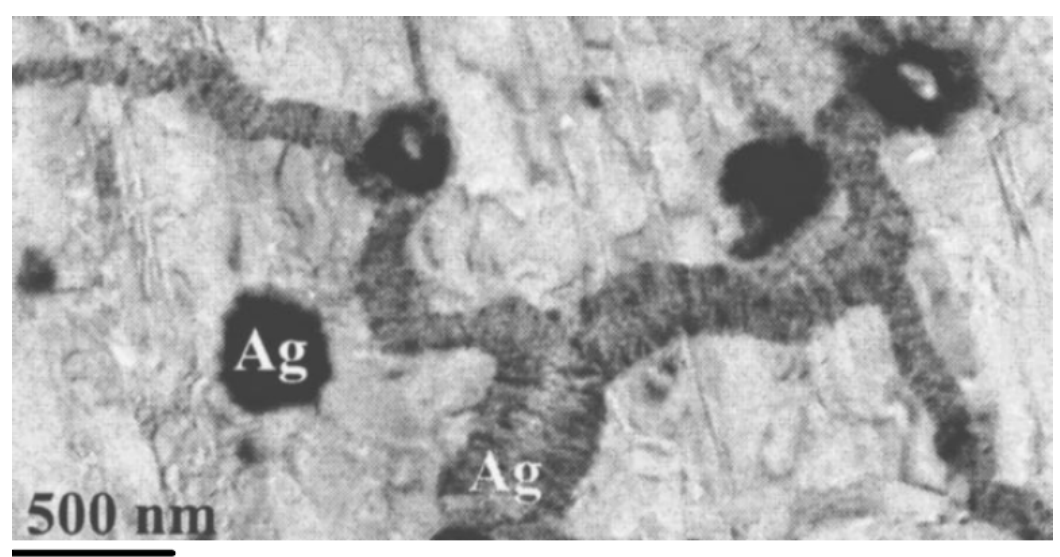

FiguRE 6.3: The microstructure of a silver chalcogenide compound showing the coexistence of silver nanoparticles and silver paths. (Reproduced from reference 24).

Figure 6.3 shows that both conducting silver branches and clusters can arise in the silver chalcogenides. If the volume fraction of silver with respect to the grain boundary and 
dislocation volume is greater than $30 \%$ (the percolation threshold) then the nanoscale silver clusters become interconnected. If the excess of silver is further increased, the volume of the grain boundaries and dislocations is completely filled thus forming a series of interconnecting silver branches. If the silver excess is increased further metallic clusters appear inside the grains. These are not connected to others and therefore have a much smaller effect on the sample's conductivity ${ }^{24}$. The formation of the silver paths and droplets is dependent on the film preparation and the excess of silver in the material $(\delta)^{2433}$. A very small excess (of the order of a few percent) of silver in the stoichiometry of the materials can produce a percolating silver network in the microstructure that increases the conductivity by more than an order of magnitude ${ }^{34}$. The appearance of the silver branches and droplets on the nanoscale in the silver chalcogenides is presented as the reason for the appearance of such a large and linear magnetoresistance effect in such materials ${ }^{33}$. The physical mechanism for both the magnetoresistance of the silver chalcogenides and the EMR effect may be related. This is suggested due to the geometrical nature of both effects in addition to similarities in system composition (e.g. a two-phase nature consisting of non-magnetic metallic and semiconducting components). However, the magnetoresistance in the silver chalcogenides increases linearly with magnetic field and does not saturate while the EMR effect observed in circular van der Pauw geometry devices is quadratic with magnetic field and tends to saturate at finite magnetic field. Subsequently, a large and linear magnetoresistance effect in a ternary matrix material, namely gold-rich $\mathrm{Ag}_{3} \mathrm{Au}_{1.1} \mathrm{Te}_{2}$ has been reported ${ }^{109}$. In this system two kinds of inhomogeneities are present (both $\mathrm{Ag}$ and $\mathrm{Au}$ ) which may enhance the possibilities of the production of materials with an inhomogenous microstructure.

\subsubsection{Previous Theoretical Approaches}

Abrikosov proposed a theoretical model of quantum magnetoresistance (QMR) in order to explain the appearance of a positive and linear magnetoresistance effect in the silver chalcogenides 35 37. This model predicts a QMR effect to arise in specific materials which are inhomogeneous on the atomic scale where a semiconducting matrix is inter-dispersed with metallic clusters. This model requires the material to be a gapless semiconductor with a linear energy spectrum. In such materials the QMR effect is positive and varies linearly with magnetic field even at room temperature and in small magnetic fields. It is suggested the presence of small excess clusters of silver in $\mathrm{Ag}_{2+\delta}$ Se may cause the QMR effect to arise $\sqrt{35}[37 / 110$.

An alternative classical explanation for the large and linear transverse magnetoresistance observed in the silver chalcogenides has been proposed by Parish and Littlewood $25[38 \mid 39$. 
Here, a two-dimensional resistor network made up of four terminal resistors was investigated numerically under the influence of a transverse magnetic field (see Figure 6.4). Four terminal resistors were required in order to account for the Hall components of the resistivity. To model a strongly inhomogeneous medium, an inhomogeneous spatial distribution of the charge carrier mobility of each of the resistors in the network was introduced to the model (a spatially varying conductivity tensor). This so-called random resistor network model has been shown to produce a similarly large and linear magnetoresistive response when there is strong disorder in the mobility and a sufficient network size ${ }^{25 \mid 38}$. The magnetic field at which the magnetoresistance switches from a quadratic to a linear dependence on magnetic field is dependent on the mobility distribution in the network. For an inhomogeneous conducting material this disorder in the mobility arises from the material's microstructure. Subsequently, the model has been extended to three-dimensions to investigate the longitudinal magnetoresistance response ${ }^{39 / 45 / 47}$. In an attempt to more realistically model the silver chalcogenides the distribution of the mobility of the resistors was changed to a quasi-random nature, with a good agreement found between the model and existing experimental data ${ }^{46}$.

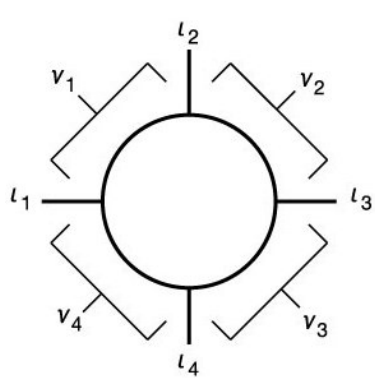

(a)

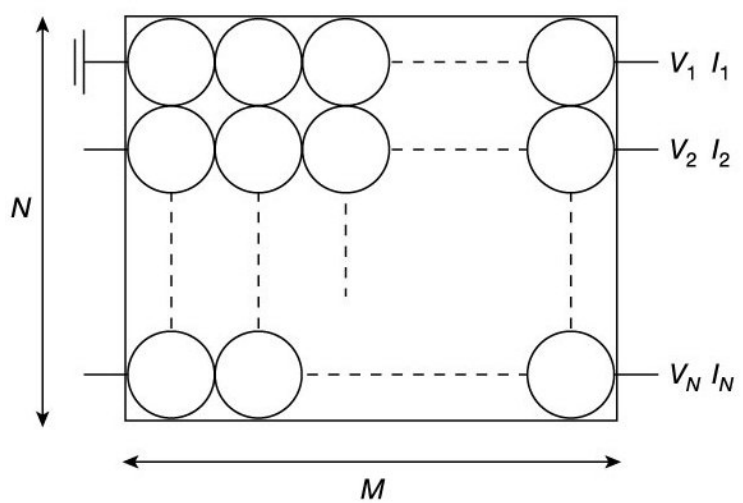

(b)

Figure 6.4: Two-dimensional four terminal resistor network of Parish and Littlewood. Showing (a) a four terminal resistor consisting of a homogeneous disk with four equally spaced contacts on the perimeter and (b) the resultant four terminal resistor network. (Adapted from reference 25).

Many other theoretical models have been proposed that exhibit magnetoresistance effects in inhomogeneous two-component systems. The appearance of a large and linear magnetoresistance effect has been shown to arise in planar and layered, strongly inhomogeneous two-phase systems by Bulgadaev and Kusmartsev는 $\underline{43}$. Three different inhomogeneous structures are considered: the random droplet model (one phase forms randomly sized droplets in the other); random parquet model (one phase forms randomly sized parquets in the other); and the effective medium model. Explicit expressions for the effective conductivity of such systems for various values of volume concentration and 
magnetic field have been obtained using the exact dual transformation and conductivity theory. The magnetoresistance of the random parquet model appears for a large range of volume concentrations while the other two models require the volume concentration to be between 0.3 and 0.5 in order to achieve the largest magnetoresistance response. It was thus suggested parquet shaped (rectangular) inclusions may be more significant than droplets in the microstructure of a material. This approach is very general and could be applied to a large range of two-phase inhomogeneous systems. A similar approach that considers the silver chalcogenides as two-dimensional two-component composites has shown a good agreement with experimental data of the silver chalcogenides ${ }^{44}$. In this case the largest magnetoresistance was obtained for a volume concentration of 0.2.

Bergman and Strelniker have also shown that composite materials with a columnar microstructure can result in a magnetoresistance effect in a strong uniform magnetic field 50 52. Guttal and Stroud have calculated the effective resistivity of a macroscopically disordered two component conductor in two-dimensions using the effective medium approximation 48149 . The obtained magnetoresistance is large and non-saturating at all non-zero values of volume concentration, with a linear dependence on magnetic field obtained when the concentration is 0.5. Magier and Bergman derived an expression for the magnetoresistance of a two-phase two-dimensional composite material when the volume concentration is equal for each phase ${ }^{53}$. In this case the magnetoresistance was linear with magnetic field, for large fields. For other values of volume concentration the magnetoresistance was found to saturate.

\subsection{Modelling in a Disordered System Geometry}

Two models have been produced with the use of the FEM in an attempt to model inhomogeneous semiconductor-metal hybrid systems. These models were based upon the microstructure of the silver chalcogenides, where a large linear magnetoresistance has been reported. Both models reported here have the same filling factor and material parameters. Since the results of these models are to be compared to the results of the silver chalcogenide systems, the material parameters and filling factor mimic those in experimental systems. The material parameters used in both of the following models can be seen in Table 6.1. The filling factor in both cases was set to $\alpha=0.32$ where only $10 \%$ of the system's area is metallic; in reasonable agreement with the discovered microstructure of the silver chalcogenides. The first model is a random branch and droplet model $(\mathrm{RBDM})$ in which randomly sized, positioned and orientated branches and droplets are created in a semiconducting material. Here, the system is similar in construction to the circular systems seen in Chapter 3, where the outer semiconducting disk is circular with 


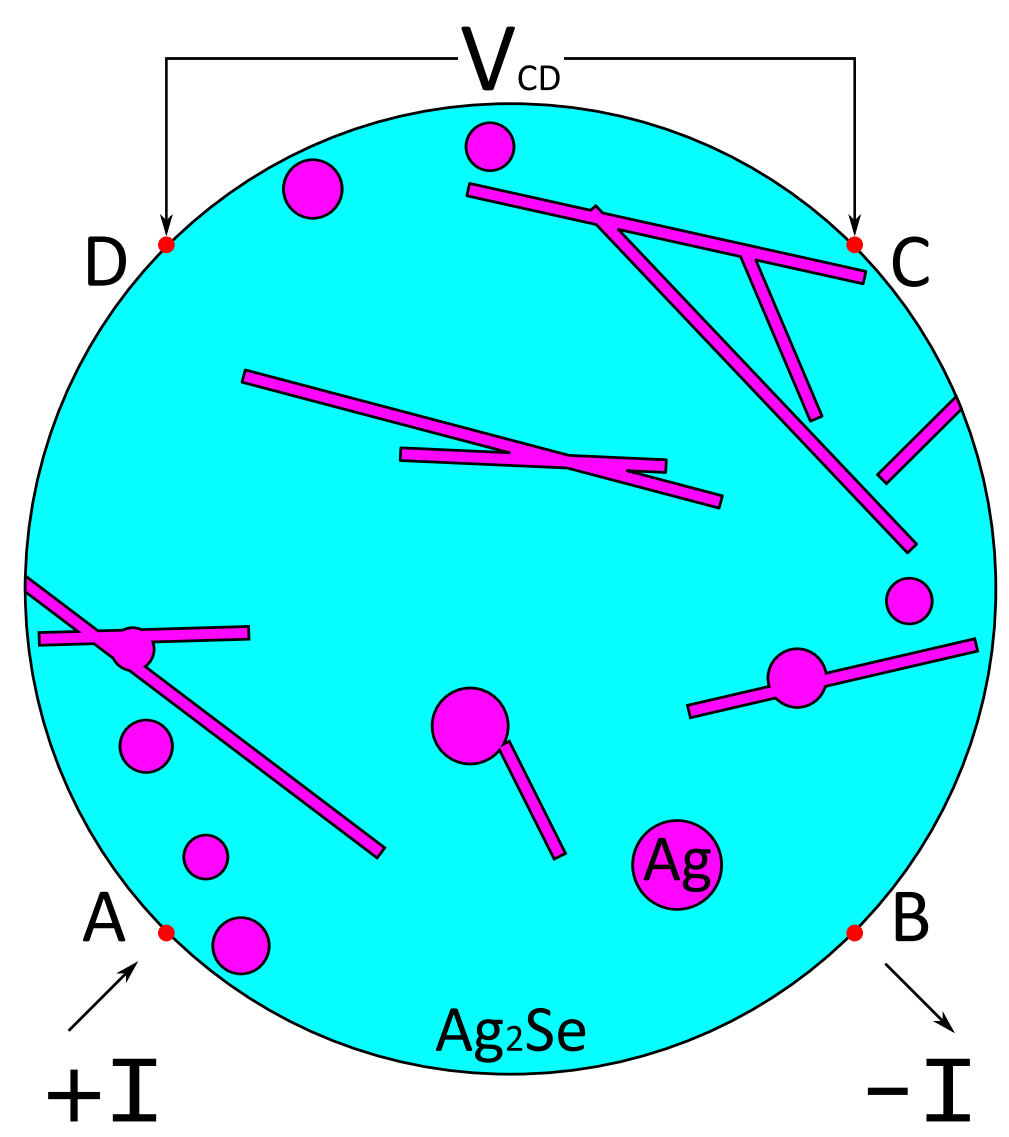

FiguRE 6.5: The system geometry of the inhomogeneous random branch and droplet model (RBDM) with filling factor $\alpha=0.32$ ( $10 \%$ of the system area is metallic). An equal area of silver metal is used to produce randomly sized, positioned and orientated branches with the other half forming randomly sized and positioned droplets in a semiconducting $\mathrm{Ag}_{2}$ Se disk (diameter of $1 \mathrm{~mm}$ ).

four contacts placed equidistant around the system perimeter. However, in this model the metallic region is formed in an inhomogeneous branch and droplet manner producing an inhomogeneous structure as shown in Figure 6.5. Here, half of the metallic area is formed of randomly sized and positioned droplets whilst the other half of the metallic inclusions comprise of randomly sized, positioned and orientated branches. The random nature of the positions of the branches and droplets forms the system geometry seen in Figure 6.5 .

The second model is another inhomogeneous semiconductor-metal system with the geometry based upon a 10x10 grid. The four contacts are placed at the corners of the square grid. In this model $10 \%$ of the squares are assigned as metallic at random, with the remaining squares semiconducting. The system geometry for this model can be seen in Figure 6.6. By randomly assigning a fraction of the squares in the $10 \times 10$ grid as metallic, with the remaining semiconducting, an inhomogeneous semiconductor-metal system can be modelled in a very general manner. 


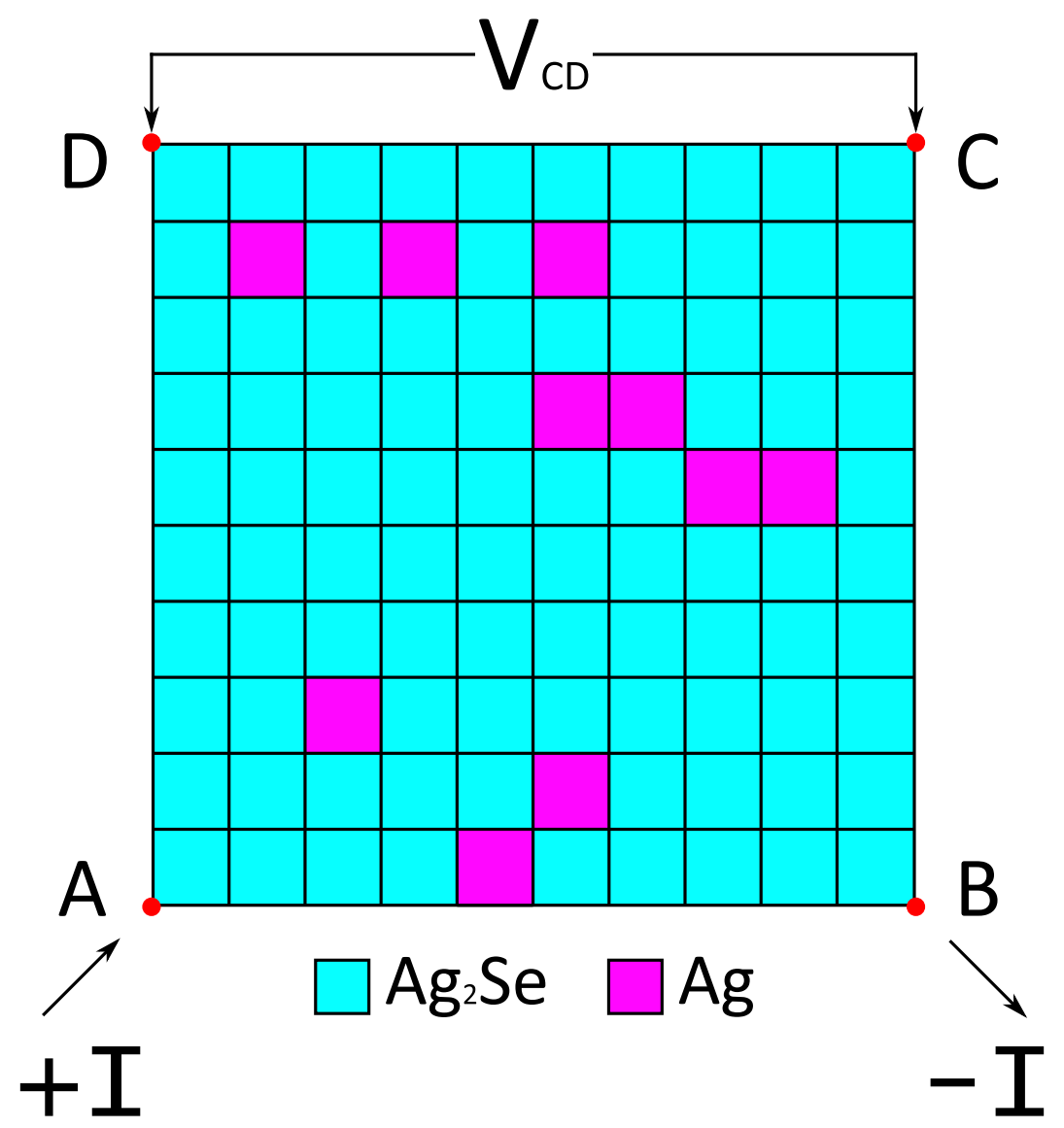

Figure 6.6: The system geometry of the inhomogeneous 10x10 grid model $(0.5 \mathrm{~mm}$ square) with filling factor $\alpha=0.32$. Here, 10 of the 100 squares are randomly assigned as silver metal with the remaining squares semiconducting $\mathrm{Ag}_{2} \mathrm{Se}$.

For both models the point settings and boundary conditions are the same as used in previous models. Namely, current set to flow between two adjacent contacts with the other two contacts used to measure the potential difference in the system. The boundary condition on the system perimeter was set to electrical insulation and the internal boundary condition was set to continuity.

The results presented in Figure 6.7 show the magnetoresistance as a function of applied magnetic field for the RBDM, 10x10 grid model and the corresponding experimental data for the silver chalcogenides. In these models the magnetic field ranges from 0 to $5.5 \mathrm{~T}$ and the material parameters are representative of those at room temperature, as in experiments. We see both models generally agree very well with the experimental data, with a quasi-linear dependence of magnetoresistance on magnetic field that continues through the entire range of magnetic field. This result is remarkable due to the very general nature of the model geometries. These results show the RBDM and 10x10 grid model are very capable of modelling the magnetoresistance that has been observed in the silver chalcogenides. In order to understand how the magnetoresistance arises in 


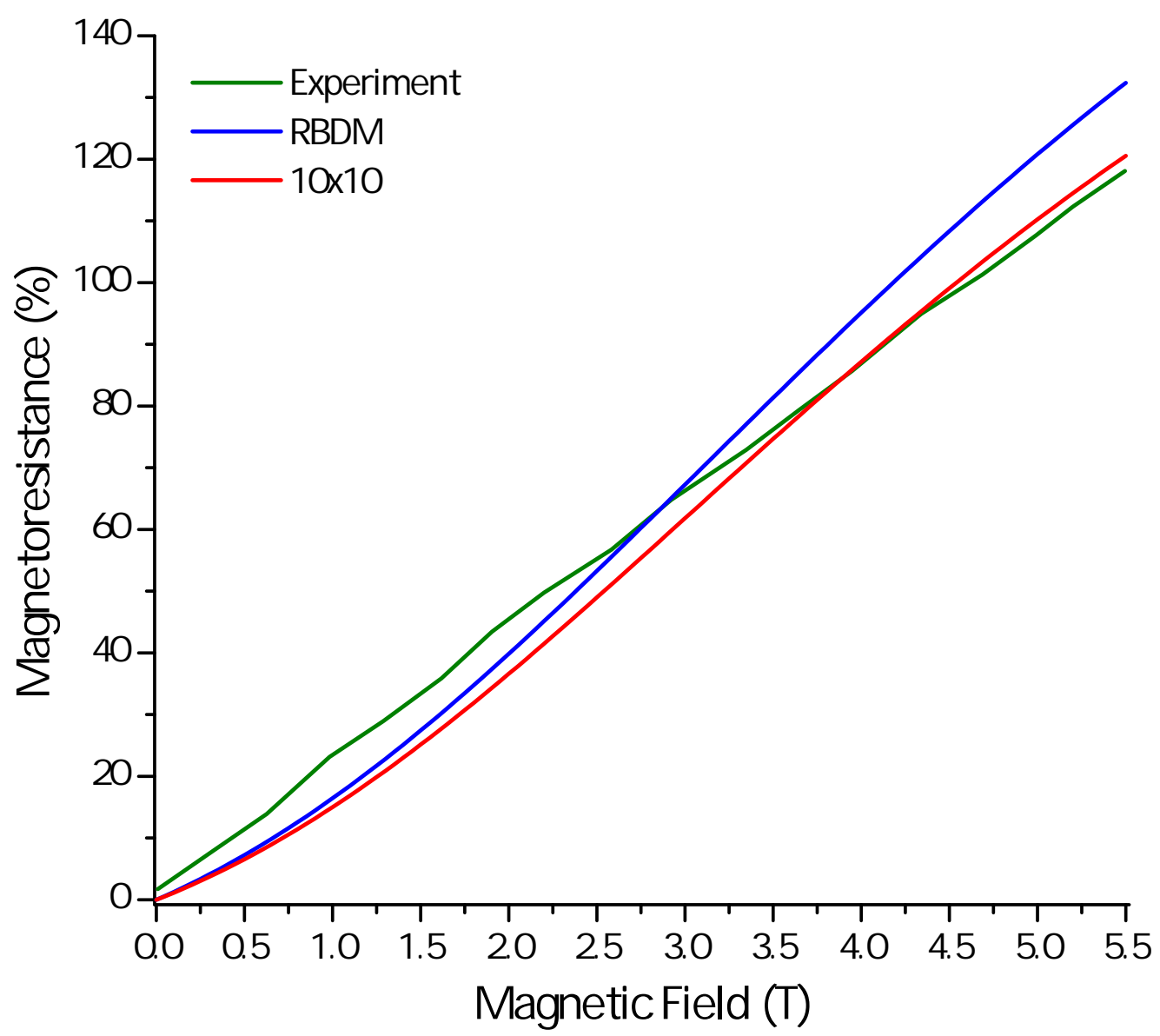

Figure 6.7: Magnetoresistance as a function of magnetic field for: the RBDM (with filling factor of $\alpha=0.32$ as seen in Figure 6.5); the 10x10 grid model (with filling factor of $\alpha=0.32$ as seen in Figure 6.6); and experimental data for the silver chalcogenides 22 .

such systems it is necessary look at the current flow throughout the systems at various values of magnetic field (0T, 3T, 5.5T and 50T). This can be seen in Figures 6.8 and 6.9 . Figures 6.8 and 6.9 generally show the same features in the current flow at a given value of magnetic field. Overall, we see that magnetic field has a large impact on the current flow in the system of both the RBDM and the 10x10 grid model. The current flow exhibited in these figures is much more complex than in the case of the circular EMR devices described in Chapter 3 which reflects the greater complexity of the system geometries over the circular disks.

Without magnetic field (Figures 6.8(a) and 6.9(a)) we see a large current flow through the metallic regions. This is seen clearly in the RBDM system when looking at the current flow into the metallic droplet closest to contact B. This is also the case in the 10x10 grid model when looking at the current flow into the metallic region closest to contact A. This is the same behaviour as in the circular EMR devices, except it occurs at many locations throughout the system. The Hall angle is zero at zero magnetic field 
and so the current tends to flow along the electric field lines; that is perpendicular to the semiconductor-metal interface and into the metal, resulting in a low system resistance.

When a very large magnetic field of $50 \mathrm{~T}$ is applied to the two systems we see a different behaviour, see Figures 6.8(d) and 6.9(d). In these figures it is apparent that the current flow is dramatically reduced in the metallic regions. The current now flows mainly through the semiconducting material and in most cases avoids the metallic regions altogether. This can be seen when looking at the current flow in the same metallic droplet in the RBDM system that is closest to contact B, whereby the current bypasses the metallic region. Similarly in the 10x10 grid model system, when observing the current flow in the vicinity of the metallic region closest to contact $A$, the current flow avoids the metallic region. This behaviour causes the system resistance in an applied magnetic field to be larger than that at zero field, thus producing a positive magnetoresistance effect.

In general this is the same effect as observed in the circular EMR system, shown in Chapter 3. Since the current flow is more complex in the RBDM and 10x10 grid models, there are some exceptions. In the RBDM we see more current flow in a 50T magnetic field in the diagonal metallic branch that is closest to contact A than observed at zero magnetic field. This appears since these systems cannot simply be thought of as many circular EMR devices found in the same system. However, the current flow around one metallic region influences the current flow around another metallic region. In this case we see that increasing magnetic field causes expulsion of current from the circular droplets near contact A, but this expulsion causes a larger proportion of current to flow through the diagonal metallic branch. This is seen much more frequently when looking at the current flow at magnetic fields of $3 \mathrm{~T}$ and $5.5 \mathrm{~T}$ respectively.

The magnetoresistance found in both the RBDM and 10x10 grid model is large but significantly lower than that observed in the systems of Solin et al. with a circular EMR device geometry. There are some important reasons for this; firstly, the semiconductor mobility in the silver chalcogenides $\left(2,500 \mathrm{~cm}^{2} / \mathrm{Vs}\right)$ is almost 20 times lower than the semiconductor used in the circular EMR devices. As we have seen in Chapter 5 this means in order to produce the same Hall angle as in the high mobility systems a much larger magnetic field is required. Therefore, the expulsion of current from the metallic regions in the RBDM and the 10x10 grid model is not as pronounced as in the circular geometry EMR systems for the same magnetic field. In order to achieve a strong expulsion of current from the metallic regions, a much larger field of approximately $50 \mathrm{~T}$ is required. In this large field, the current expulsion is more apparent and can be seen in Figures 6.8(d) and 6.9 (d). Secondly, the filling fraction in the silver chalcogenides, RBDM and the $10 \times 10$ grid model is relatively low $(\alpha=0.32)$. From Chapter 3 it was shown that in general larger filling factors result in larger magnetoresistance effects. 


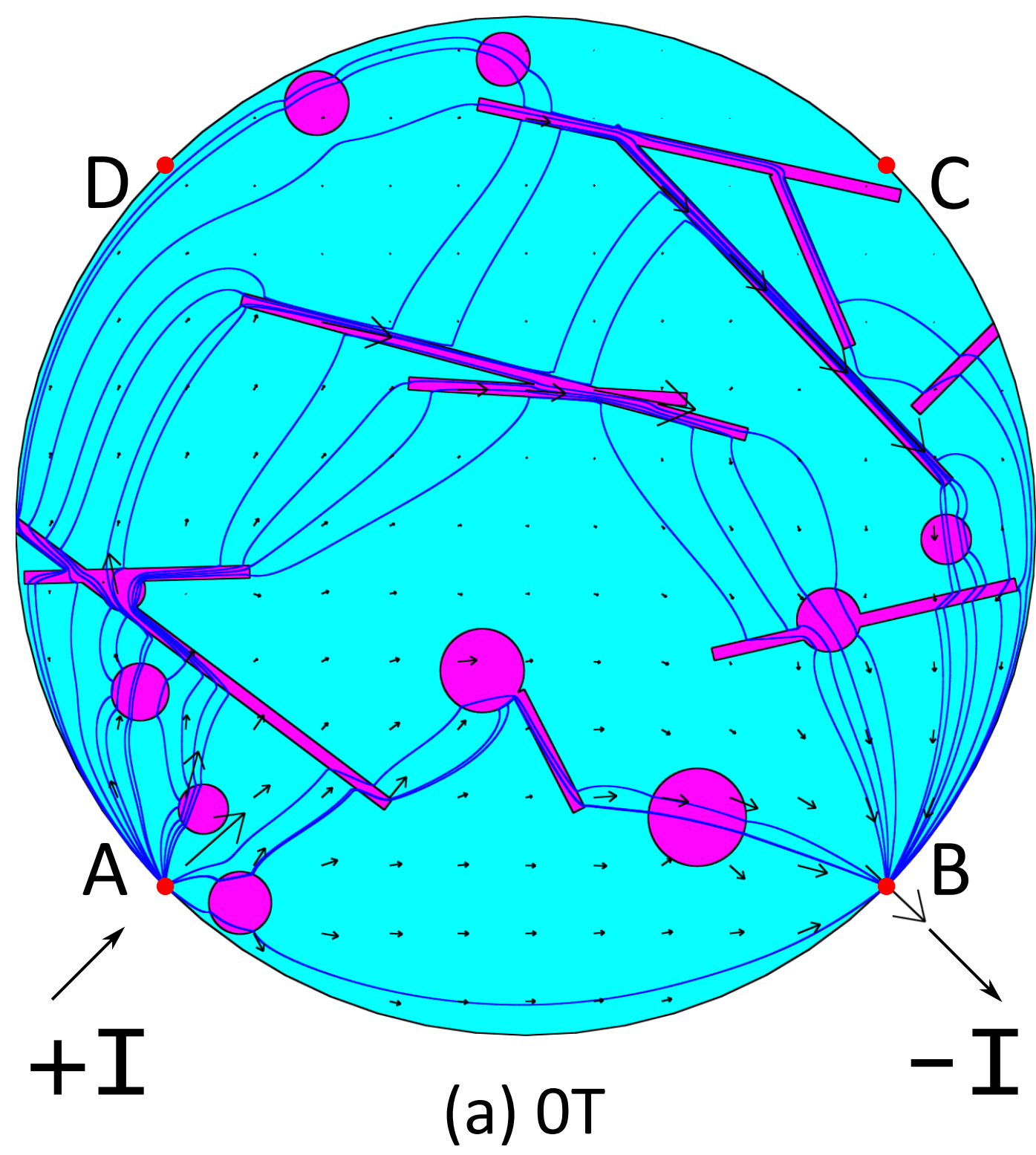

Figure 6.8: Visualisation of the current flow throughout a RBDM system (with filling factor of $\alpha=0.32$ as seen in Figure 6.5 produced via modelling in Comsol Multiphysics. The background colour represents the two phases, silver metal (pink) and semiconducting $\mathrm{Ag}_{2} \mathrm{Se}$ (cyan). The current density is represented by both the blue streamlines and black arrows. The streamlines connect regions with the same current density, while the arrows show the magnitude and direction of the current flow at various points throughout the system. The four images in this figure represent different applied magnetic fields: (a) $0 \mathrm{~T}$; (b) 3T; (c) $5.5 \mathrm{~T}$; and (d) 50T. 


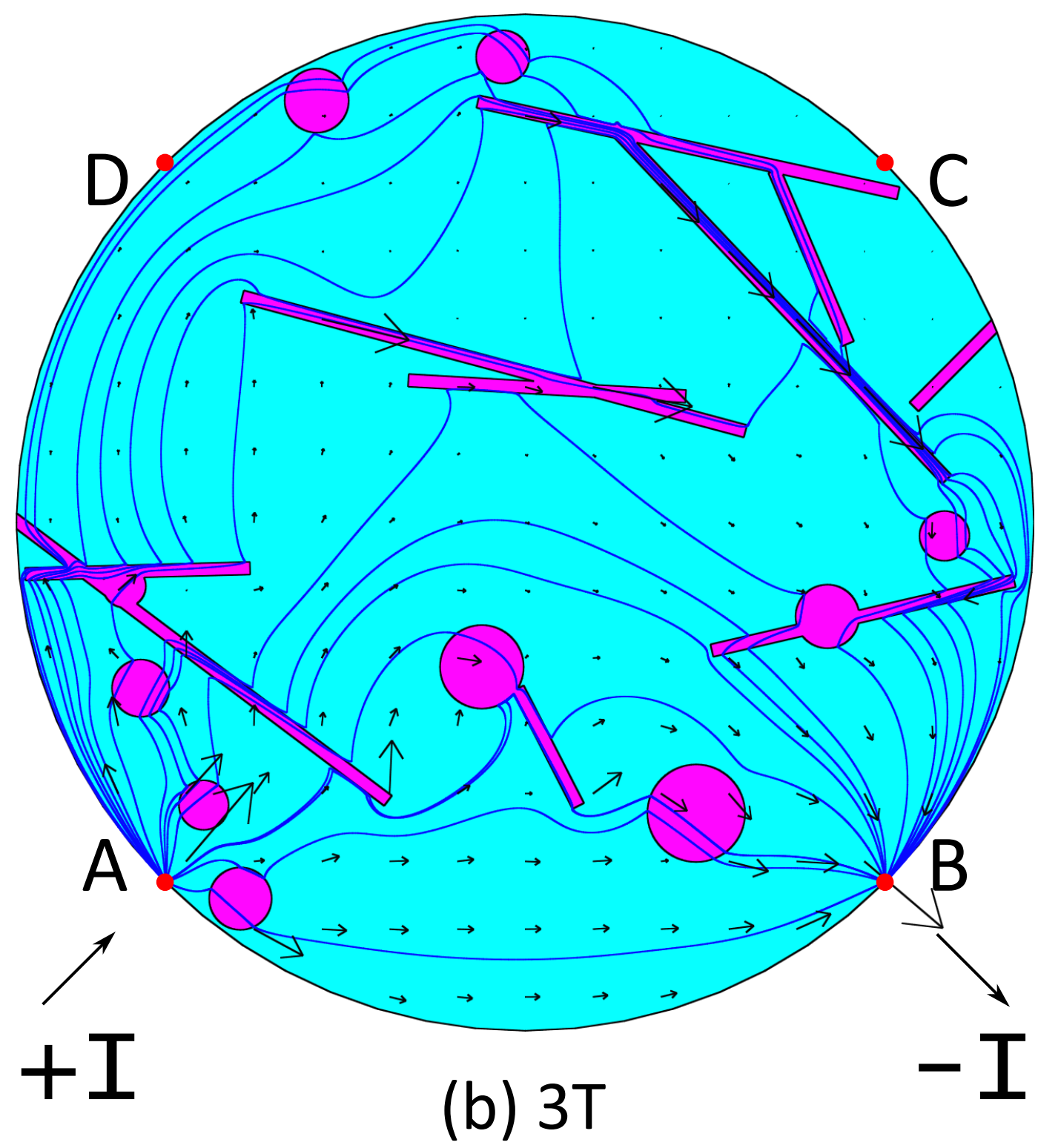

Figure 6.8: (Cont.) Visualisation of the current flow throughout a RBDM system (with filling factor of $\alpha=0.32$ as seen in Figure 6.5 produced via modelling in Comsol Multiphysics. The background colour represents the two phases, silver metal (pink) and semiconducting $\mathrm{Ag}_{2} \mathrm{Se}$ (cyan). The current density is represented by both the blue streamlines and black arrows. The streamlines connect regions with the same current density, while the arrows show the magnitude and direction of the current flow at various points throughout the system. The four images in this figure represent different applied magnetic fields: (a) 0T; (b) 3T; (c) 5.5T; and (d) 50T. 


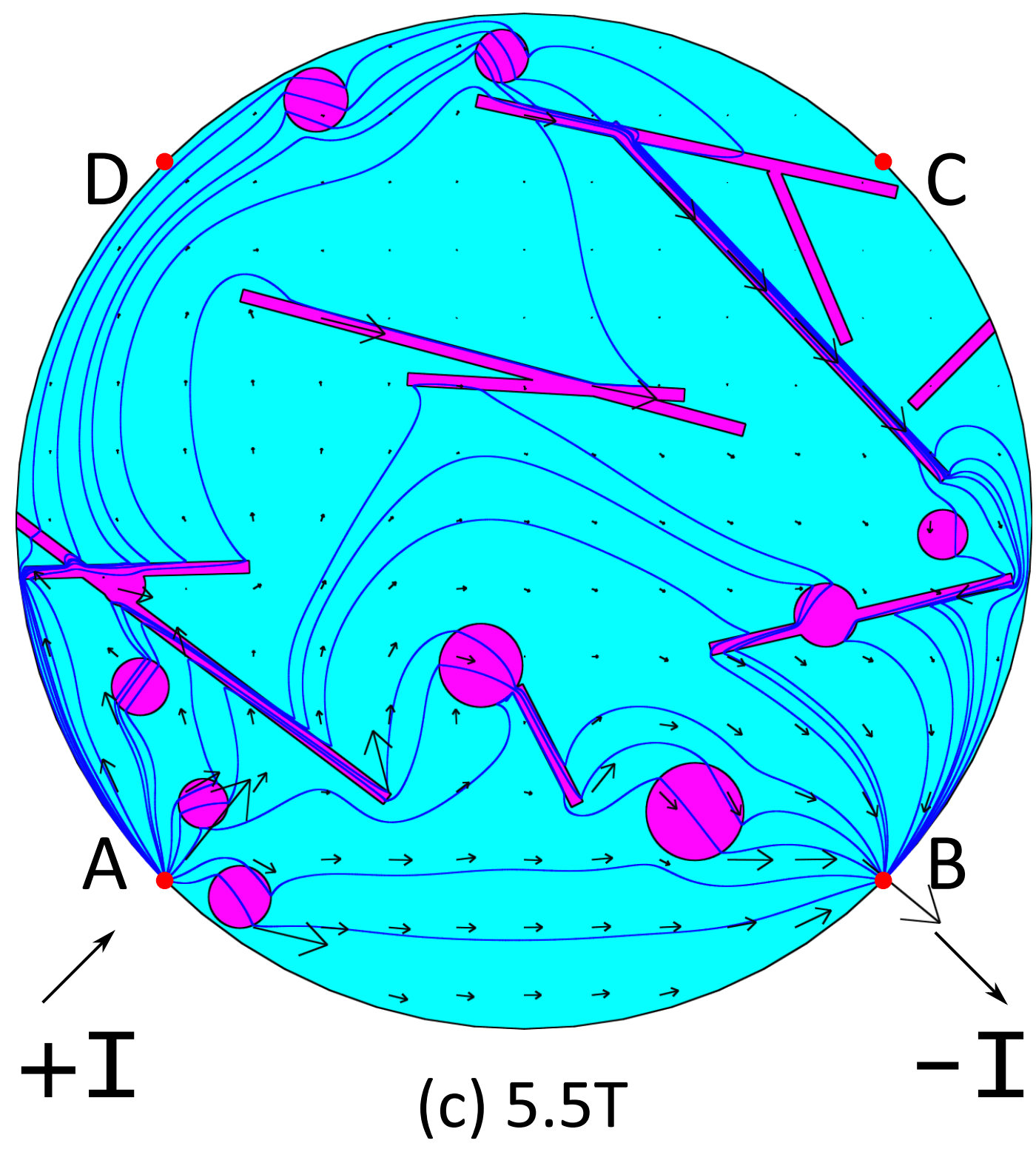

FiguRE 6.8: (Cont.) Visualisation of the current flow throughout a RBDM system (with filling factor of $\alpha=0.32$ as seen in Figure 6.5 produced via modelling in Comsol Multiphysics. The background colour represents the two phases, silver metal (pink) and semiconducting $\mathrm{Ag}_{2} \mathrm{Se}$ (cyan). The current density is represented by both the blue streamlines and black arrows. The streamlines connect regions with the same current density, while the arrows show the magnitude and direction of the current flow at various points throughout the system. The four images in this figure represent different applied magnetic fields: (a) 0T; (b) 3T; (c) 5.5T; and (d) 50T. 


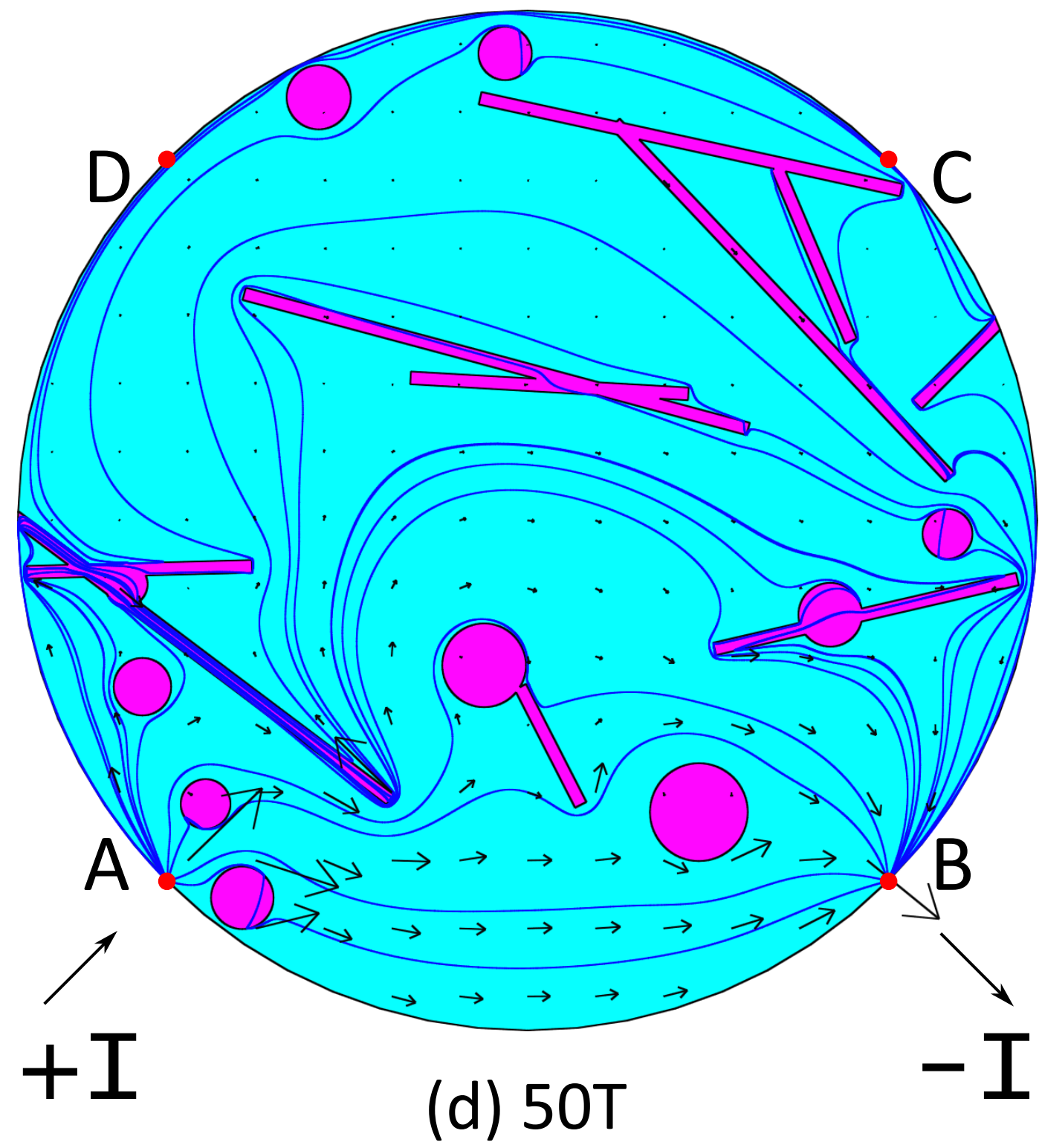

Figure 6.8: (Cont.) Visualisation of the current flow throughout a RBDM system (with filling factor of $\alpha=0.32$ as seen in Figure 6.5 produced via modelling in Comsol Multiphysics. The background colour represents the two phases, silver metal (pink) and semiconducting $\mathrm{Ag}_{2} \mathrm{Se}$ (cyan). The current density is represented by both the blue streamlines and black arrows. The streamlines connect regions with the same current density, while the arrows show the magnitude and direction of the current flow at various points throughout the system. The four images in this figure represent different applied magnetic fields: (a) 0T; (b) 3T; (c) 5.5T; and (d) 50T. 


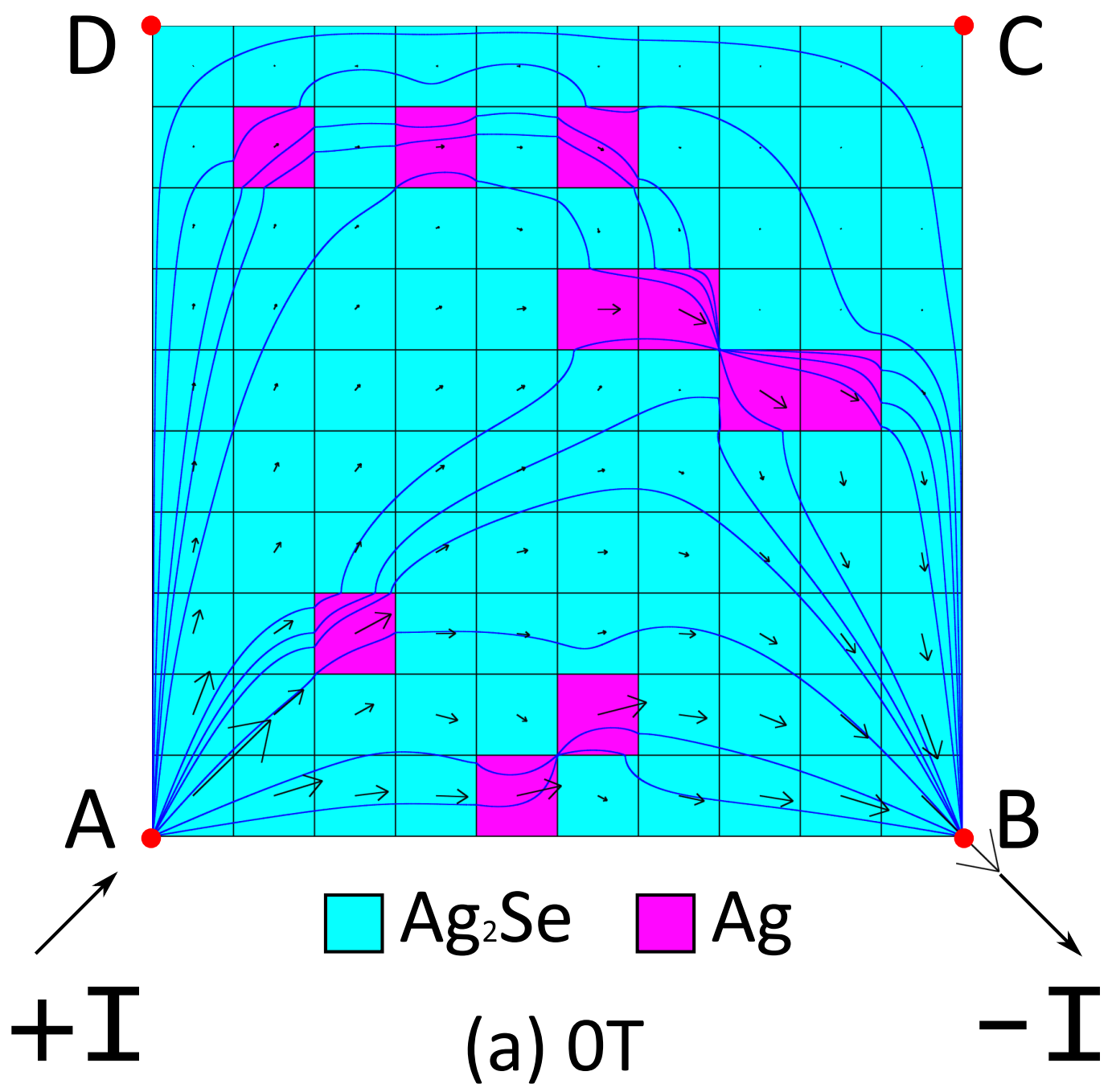

FIGURE 6.9: Visualisation of the current flow throughout a 10x10 grid model system (with filling factor of $\alpha=0.32$ as seen in Figure 6.6) produced via modelling in Comsol Multiphysics. The background colour represents the two phases, silver metal (pink) and semiconducting $\mathrm{Ag}_{2} \mathrm{Se}$ (cyan). The current density is represented by both the blue streamlines and black arrows. The streamlines connect regions with the same current density, while the arrows show the magnitude and direction of the current flow at various points throughout the system. The four images in this figure represent different applied magnetic fields: (a) 0T; (b) 3T; (c) 5.5T; and (d) 50T. 


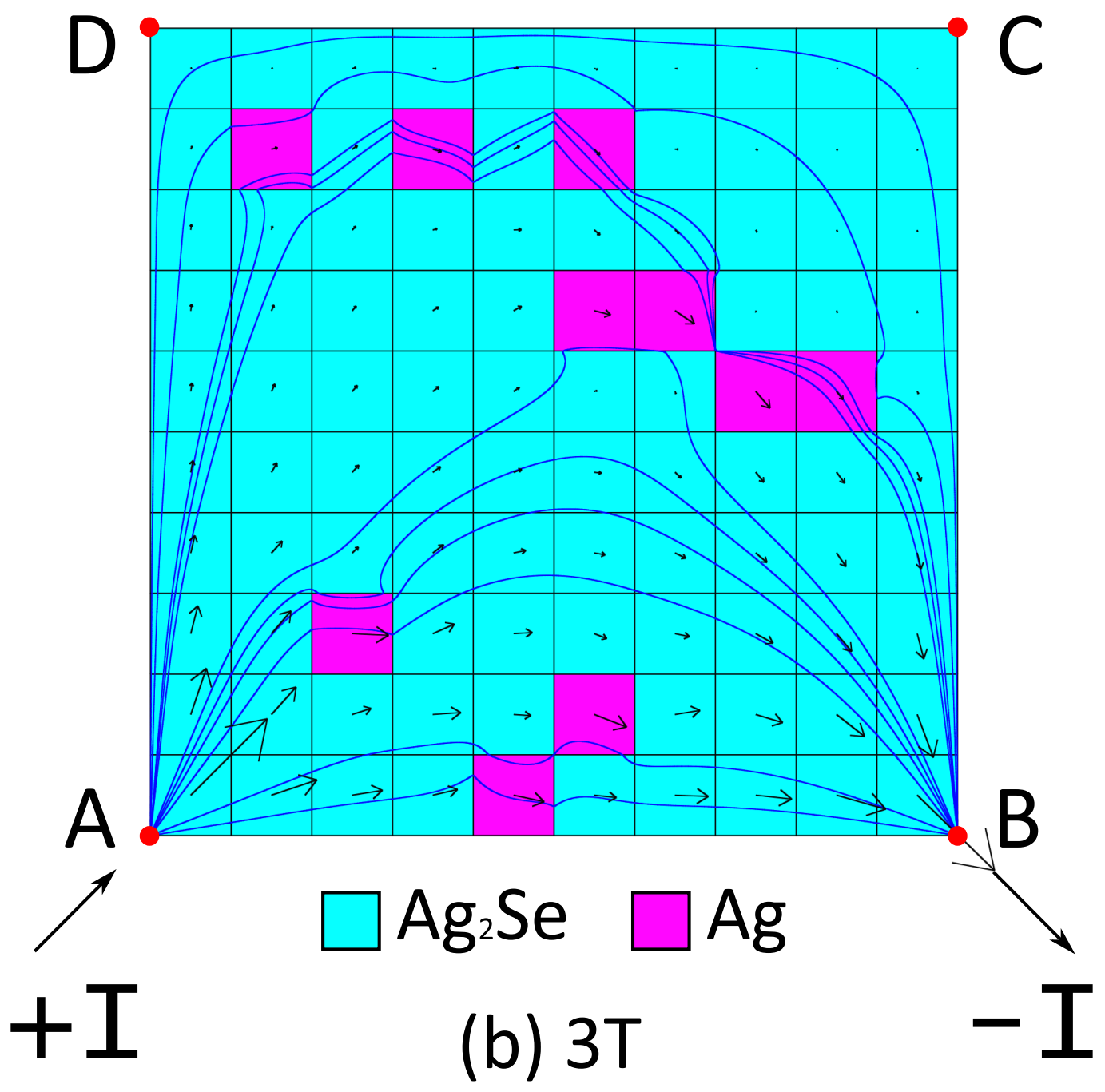

Figure 6.9: (Cont.) Visualisation of the current flow throughout a 10x10 grid model system (with filling factor of $\alpha=0.32$ as seen in Figure 6.6 produced via modelling in Comsol Multiphysics. The background colour represents the two phases, silver metal (pink) and semiconducting $\mathrm{Ag}_{2} \mathrm{Se}$ (cyan). The current density is represented by both the blue streamlines and black arrows. The streamlines connect regions with the same current density, while the arrows show the magnitude and direction of the current flow at various points throughout the system. The four images in this figure represent different applied magnetic fields: (a) 0T; (b) 3T; (c) 5.5T; and (d) 50T. 


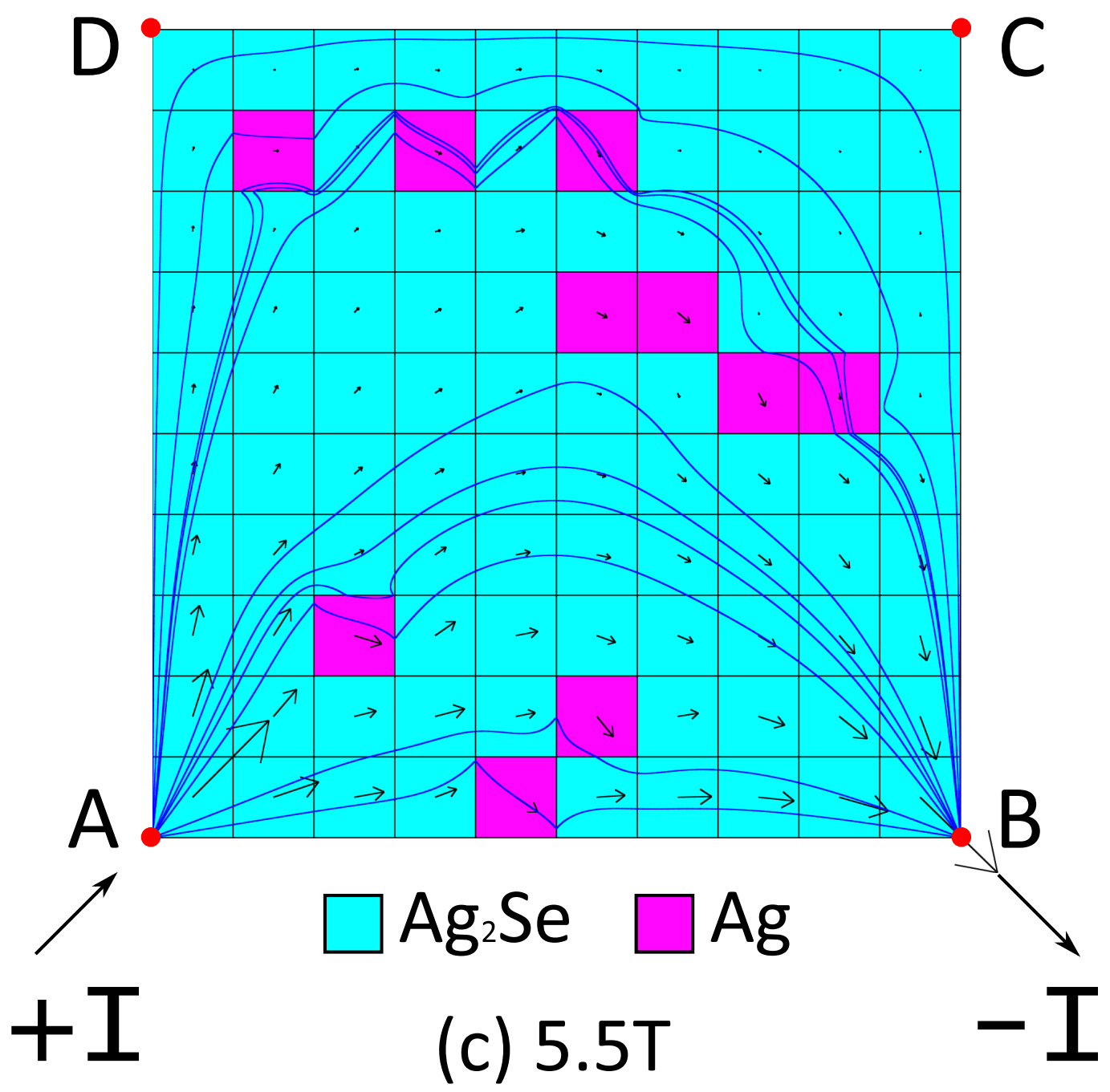

Figure 6.9: (Cont.) Visualisation of the current flow throughout a 10x10 grid model system (with filling factor of $\alpha=0.32$ as seen in Figure 6.6 produced via modelling in Comsol Multiphysics. The background colour represents the two phases, silver metal (pink) and semiconducting $\mathrm{Ag}_{2} \mathrm{Se}$ (cyan). The current density is represented by both the blue streamlines and black arrows. The streamlines connect regions with the same current density, while the arrows show the magnitude and direction of the current flow at various points throughout the system. The four images in this figure represent different applied magnetic fields: (a) 0T; (b) 3T; (c) 5.5T; and (d) 50T. 


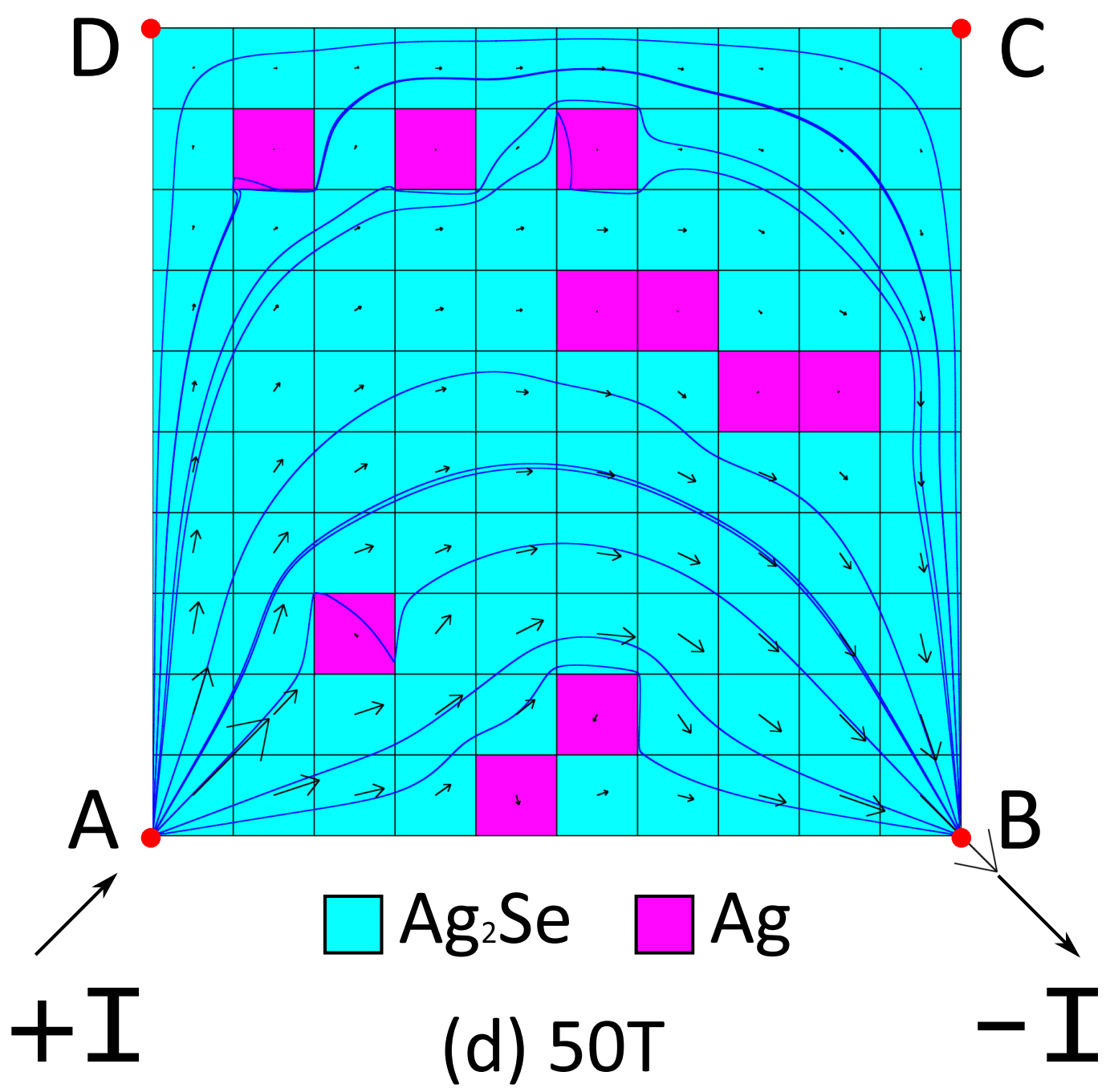

Figure 6.9: (Cont.) Visualisation of the current flow throughout a 10x10 grid model system (with filling factor of $\alpha=0.32$ as seen in Figure 6.6 produced via modelling in Comsol Multiphysics. The background colour represents the two phases, silver metal (pink) and semiconducting $\mathrm{Ag}_{2} \mathrm{Se}$ (cyan). The current density is represented by both the blue streamlines and black arrows. The streamlines connect regions with the same current density, while the arrows show the magnitude and direction of the current flow at various points throughout the system. The four images in this figure represent different applied magnetic fields: (a) 0T; (b) 3T; (c) 5.5T; and (d) 50T. 
Figures 6.8(b), 6.8(c), 6.9(b) and 6.9(c) show the current flow in the two systems when in intermediate fields; that is, fields at which the resultant Hall angle is significantly larger than zero but is far away from approaching $90^{\circ}$. In this situation the current flow is in a transitional state between that observed at zero field, where the metallic regions had a large amount of current flowing through them, and the case at very high field where the current in general avoided the metallic regions. In the RBDM this behaviour appears as the increase in the angle of the current flow at the interface between semiconductor and metal. This can be seen in the metallic droplet closest to contact B in Figures 6.8(b) and 6.8 (c). The magnetic field is not strong enough to expel the current from the metallic region but the current flow is affected, resulting in a larger proportion of the current flowing in the semiconducting material than at zero field. In the 10x10 grid model this behaviour can be seen in the three metallic regions nearest the top of the system. The current flow through these regions forms zigzag patterns. The magnetic field is not strong enough to completely expel the current but instead influences its path causing a larger proportion of current to flow through the semiconducting material.

The structure of the RBDM and 10x10 grid system is inhomogeneous and far removed from the symmetrical circular EMR devices. This may be a reason for the observed quasi-linear dependence of the magnetoresistance on magnetic field. This linear nature could be explained by the combination of the effect of many metallic droplets in the same system. As we have seen from Figures 6.8 and 6.9, the current flow in one metallic droplet appears to be influenced by the current flow of the other droplets in the system. An increase in magnetic field does not lead to expulsion of current from all metallic regions, in fact the current flow in a single metallic region may increase due to the changes in current flow in its vicinity. Therefore, the increase in magnetoresistance is not as rapid as for circular EMR devices. This could explain the quasi-linear dependence of the magnetoresistance on magnetic field.

In order to reinforce the patterns seen in the current flow in Figures 6.8 and 6.9 the current along a small cross-section in both the RBDM and 10x10 grid models can be seen in Figures 6.10 and 6.11 respectively. In Figure 6.10 the current flow through a single circular droplet is shown for two values of magnetic field, 0T and 50T. At zero magnetic field the current is a maximum between points 2 and 3 in the cross-section. This region corresponds to the conducting droplet where a large current flows. However, the expulsion of current from this conducting region is clear at $50 \mathrm{~T}$ where the current flow in the semiconducting material, in the vicinity of the droplet, is much larger than inside the metallic region itself.

Similarly, in Figure 6.11 the current flow through a single square conducting region is shown for two values of magnetic field, $0 \mathrm{~T}$ and 50T. Here, at zero magnetic field the 

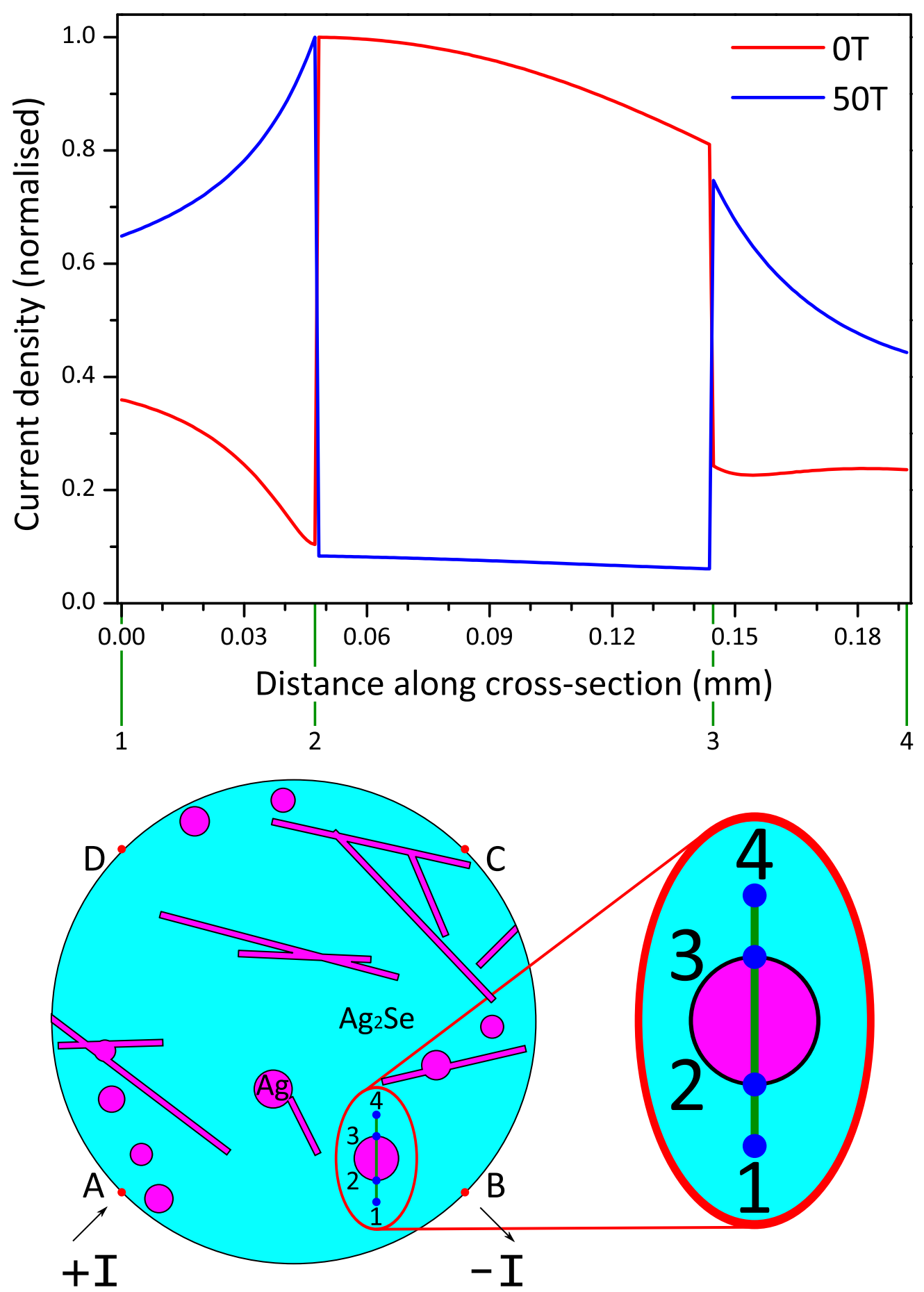

Figure 6.10: The total current density (normalised) along a small cross-section of a RBDM system (as seen in Figure 6.5) for two values of magnetic field, 0T and 50T. Here, points 1 to 4 on the vertical cross-section correspond to points 1 to 4 on the $\mathrm{x}$ axis of the plot. 

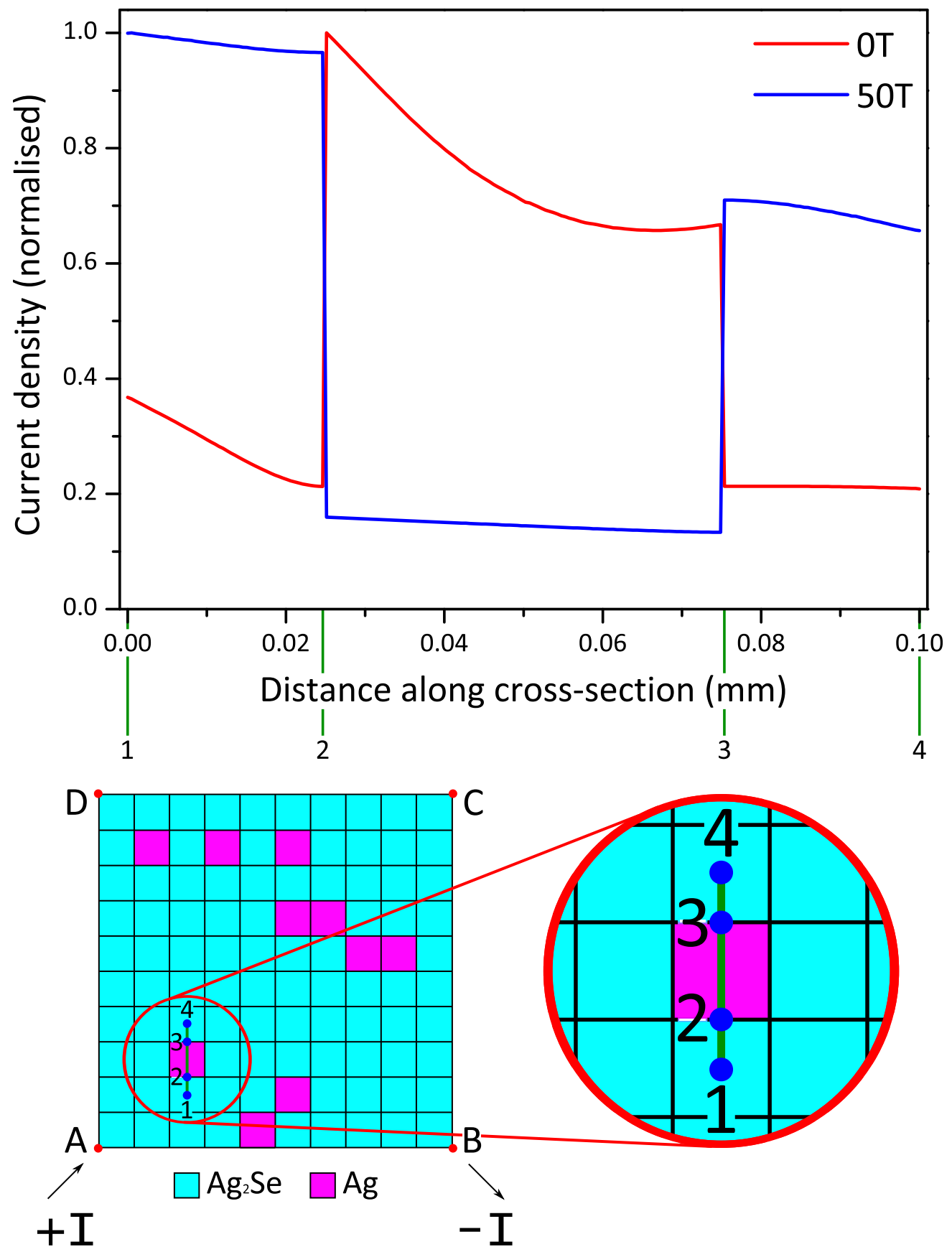

Figure 6.11: The total current density (normalised) along a small cross-section of a 10x10 grid model system (as seen in Figure 6.5 for two values of magnetic field, 0T and 50T. Here, points 1 to 4 on the vertical cross-section correspond to points 1 to 4 on the $\mathrm{x}$ axis of the plot. 
current flow is greatest inside the metallic region (between points 2 and 3 ) as shown in the current flow diagram in Figure 6.8(a). When a 50T magnetic field is applied the current is once again seen to be much greater around the conducting region than inside. This expulsion of current is the basis of the magnetoresistance in both the RBDM and the 10x10 grid models, with the switching between the low and high field extremes occurring at intermediate magnetic fields. Both Figures 6.10 and 6.11 show similar behaviour, this is much the same as the cross-section for an individual circular EMR device seen in Figure 3.17 .

Since the structure of both the RBDM and 10x10 grid model were determined by the use of random numbers, alternative configurations should exhibit a similar behaviour. Figures 6.12(i) and (j) show the magnetoresistance against magnetic field for multiple configurations (Figures 6.12(a) - (h)) of the RBDM and 10x10 grid model. All of the configurations here have the same filling factor $(\alpha=0.32)$ and use the material parameters of the silver chalcogenide materials, as given in Table 6.1. In Figures 6.12(i) and (j), we see that the magnitude of the quasi-linear magnetoresistance observed for both the RBDM and 10x10 grid model is influenced by the specific system configuration in each case. For the RBDM system the average magnitude of the magnetoresistance in a magnetic field of $5.5 \mathrm{~T}$ is $136.7 \%$ with a standard deviation of $29.1 \%$. While the $10 \mathrm{x} 10$ grid model exhibits a mean magnetoresistance at $5.5 \mathrm{~T}$ of $115.1 \%$ with a standard deviation of $17.3 \%$. This spead in magnetoresistance results from the variety observed in the structure of each system configuration in Figure 6.12. Since each configuration has a complex system geometry a complex current flow will result. The complexity of the current flow means that upon the application of a magnetic field the expulsion of current from one conducting region may cause current to flow through another conducting region that did not carry much current at zero field. This causes the magnetoresistance to increase at a slower rate than in the case of circular EMR devices and results in the observed magnetoresistance having a quasi-linear dependence on magnetic field. For much larger systems (containing many more conducting inclusions and far away from the single droplet-like case of Solin et al.) the resultant magnetoresistance would be expected to increase linearly with magnetic field due to the averaging of the effects of all of the conducting regions.

The two inhomogeneous models considered in this Thesis are extremely general and can therefore be applied to model the magnetoresistance in any two-phase system where one component has a significantly larger conductivity than the other. These models have shown the large linear magnetoresistance of the silver chalcogenides can be explained due to an inhomogeneous distribution of conducting silver in a semiconducting $\mathrm{Ag}_{2} \mathrm{Se}$ material. 

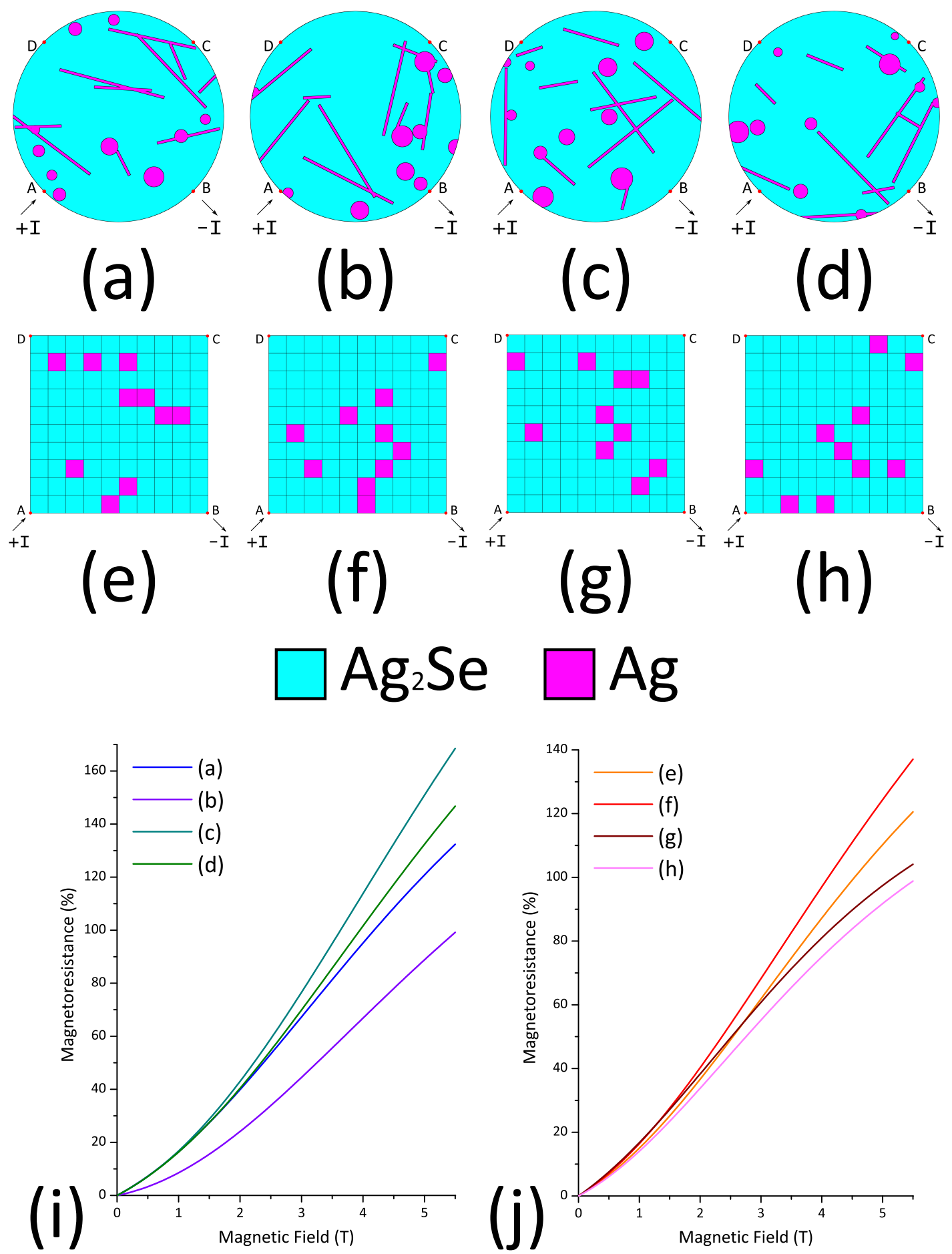

FiguRE 6.12: Multiple configurations of the RBDM (see (a) - (d)) and the 10x10 grid model (see (e) - (h)) presented alongside the corresponding magnetoresistance for each case as a function of magnetic field (see (i) and (j) for the RBDM and 10x10 grid model respectively). Configurations (a) and (e) are the ones that have been investigated in more detail in this chapter, as seen in Figures 6.5 and 6.6. All configurations have a

filling factor of $\alpha=0.32$ and the same material parameters as given in Table 6.1 


\section{Chapter 7}

\section{Conclusion}

This Thesis has numerically investigated the effect of extraordinary magnetoresistance (EMR) as well as the magnetoresistance of disordered semiconductor-metal hybrids with the use of the finite element method (FEM). Models of such inhomogeneous semiconductormetal systems were created using the Comsol Multiphysics software which has allowed for specific aspects of these systems to be investigated.

The first two chapters introduced the topic of magnetoresistance in semiconductor-metal hybrids and established a background knowledge essential for the work reported in subsequent chapters. An overview of previously discovered forms of magnetoresistance is presented, which places the EMR effect in context. Electron transport under the influence of a transverse magnetic field is considered with the introduction of the magnetoconductivity tensor and a brief account of the Hall effect provided. Finally, the FEM is discussed including an overview of the steps required in order to produce such a model using Comsol Multiphysics.

Chapter 3 reviews research on the EMR effect including experimental data from multiple sources. The circular geometry systems in which the effect was discovered are reviewed and the modelling procedure required to create a realistic model of such systems including the simplifications necessary to do so are discussed. Results of the models are then presented with the magnetoresistance plotted as a function of both magnetic field and filling factor. Generally, these models are in very good agreement with existing experimental data considering their limitations. The simulations tend to be an overestimate of the experimentally measured magnetoresistance, which is attributed to the deviation of the models from the experimental systems. The models produce a clear visualisation of the process by which the magnetoresistance arises in such systems by looking at the current flow at various values of magnetic field. The expulsion of current from the metallic region 
upon the application of magnetic field and the appearance of the Hall angle is clearly observed.

Chapter 4 advances the existing model by investigating the influence of the geometry of the conducting region on the magnetoresistance. Here, a review is given of previous alterations to the circular EMR device geometry, including the production of linear device geometries with the use of conformal mapping and different contact configurations that have been shown to enhance the magnetoresistance. The effect of the shape of the conducting region on the magnetoresistance was then investigated in 9 systems, each with a different shape of metallic component. The magnetoresistance of EMR devices was found to be extremely sensitive to the geometry of the conducting region, with a multibranched conducting region producing the largest enhancement to the magnetoresistance. This enhancement reached over four orders of magnitude when compared to a device with a traditional circular metallic region of the same filling factor at a magnetic field of $5 \mathrm{~T}$. This geometrical enhancement may be practically significant as it could be used to offset the effect of poor material parameters and thus expand the number of potential materials that could be used to produce effective future EMR sensors. Reviewing the current distribution throughout this multi-branched system, the nature of the geometrical enhancement is clearly observed. Using conformal mapping, circular geometry EMR devices have been shown to transform to an equivalent linear device geometry. Such a linear EMR device has been modelled and, as expected, the EMR mechanism is shown to arise.

In Chapter 5 the effect on the magnetoresistance of varying the material parameters of circular geometry EMR devices was investigated. Initially, results from previous studies into the effect of changing material parameters in linear EMR devices were reviewed. The specific material parameters investigated were: the mobility of the semiconducting material; the ratio of the conductivity of the metallic to semiconducting regions; and the effect of a finite resistance introduced at the semiconductor-metal interface (modelled by the introduction of a thin intermediate layer at the interface where the conductivity is not dependent on magnetic field). Investigation into these parameters has led to the discovery of a range of optimal values in which future EMR devices should adhere in order for the observation of a significant EMR effect. Firstly, the conductivity of the metallic region is required to be two orders of magnitude or greater than the conductivity of the semiconducting region. In addition, the charge carrier mobility of the semiconducting region is required to be large as it has been shown to strongly influence the magnetoresistance. Finally, a small contact resistance at the semiconductor-metal interface is required as it was found to reduce the observed magnetoresistance; vanishing altogether for large values. The introduction of a finite interface resistance has produced a model that is more realistic when compared to the experimental systems. Using the 
interface resistance as a parameter, the model was fitted to experimental data and the contact resistance of the system estimated.

Chapter 6 departs from traditional EMR systems and focuses on magnetoresistance arising in more complex and disordered structures. Here, the unusual large, linear and unsaturating magnetoresistance observed in the silver-rich silver chalcogenides is reviewed along with many proposed theoretical models for the observed magnetoresistance effect. This Thesis offers two new models in order to describe general two-phase disordered semiconductor-metal hybrid systems. Both models have been shown to produce a similarly large and quasi-linear magnetoresistance as observed in the silver chalcogenides. The first model is the random branch and droplet model (RBDM) where a small proportion of metal in the system was formed into circular droplets and rectangular bars that have randomly distributed sizes, positions and orientations. The model was based upon the microstructure of silver-rich silver chalcogenides. The second model is similar in construction to the random resistor network model of Parish and Littlewood. Here, a 10x10 square grid has a random distribution of metallic squares in a semiconducting material ${ }^{25}$. Both models show very good agreement with existing experimental data from silver-rich silver chalcogenide materials for the same material parameters.

The work presented in this Thesis provides an increased understanding of the EMR effect, specifically investigating the mechanism behind the effect and how it is influenced by changes in system geometry and material parameters. This research highlights the potential for future production of EMR devices from a larger range of materials than those currently considered. Consequently, EMR devices could be produced more readily with existing manufacturing processes in a more cost-effective manner. In considering a two-phase semiconductor-metal system with a highly disordered structure it has been shown that magnetoresistance can increase linearly with magnetic field, which is also a desirable property for practical magnetoresistive sensors. The EMR effect has generated much interest since its discovery due to the huge potential for improvements in magnetic field sensors across many fields. One such development lies in the production of the next generation of hard disk drives, where smaller more sensitive magnetic field sensors are required in the ongoing search for larger data densities. Future research into the EMR effect is required if it is to be realised in future practical devices. The modelling techniques presented in this Thesis provide a highly adaptable framework which can be modified to meet any potential system requirements. Therefore, further FEM modelling of EMR systems could optimise the performance of EMR devices. This optimisation could investigate the effect of the shape of the device and positioning of the electrical contacts. There is also potential to produce an experimental system containing a metallic region with a multi-branched structure in order to further investigate the geometrical enhancement reported here. 


\section{Appendix A}

\section{The van der Pauw Method}

The van der Pauw method has become a standard technique for the practical measurement of the sheet resistance of a thin film material ${ }^{28}$. It can be used to determine the sheet resistance of a flat conducting sample of an arbitrary shape if the following conditions are satisfied $26 \mid 27$ :

- The contacts are at the circumference of the sample;

- The contacts are sufficiently small;

- The sample is homogeneous in thickness; and

- The surface of the sample is singly connected i.e. the sample does not have any isolated geometric holes.

In this technique, four small successive ohmic contacts (A, B, C and D) are placed on the surface of the sample at arbitrary places along the circumference (see Figure A.1(a) Points A and B serve as a source and sink for an applied current $\left(I_{\mathrm{AB}}\right)$ with contacts $\mathrm{C}$ and $\mathrm{D}$ used to measure the voltage drop $\left(V_{\mathrm{CD}}=V_{\mathrm{C}}-V_{\mathrm{D}}\right)$. This allows for the resistance $R_{\mathrm{AB}, \mathrm{CD}}$ to be defined as

$$
R_{\mathrm{AB}, \mathrm{CD}}=\frac{\left|V_{\mathrm{CD}}\right|}{I_{\mathrm{AB}}},
$$

similarly we can define $R_{\mathrm{BC}, \mathrm{DA}}$

$$
R_{\mathrm{BC}, \mathrm{DA}}=\frac{\left|V_{\mathrm{DA}}\right|}{I_{\mathrm{BC}}} .
$$

\footnotetext{
${ }^{*}$ The notation differs from that of the original papers but is consistent with the work in this Thesis.
} 
The van der Pauw measurement method is based on the following relationship between $R_{\mathrm{AB}, \mathrm{CD}}$ and $R_{\mathrm{BC}, \mathrm{DA}}$

$$
\exp \left(\frac{-\pi d}{\rho} R_{\mathrm{AB}, \mathrm{CD}}\right)+\exp \left(\frac{-\pi d}{\rho} R_{\mathrm{BC}, \mathrm{DA}}\right)=1
$$

Here, $\mathrm{d}$ is the thickness of the sample and $\rho$ is the resistivity of the material. The resistivity of the material can therefore be determined if the sample thickness and the two resistances $R_{\mathrm{AB}, \mathrm{CD}}$ and $R_{\mathrm{BC}, \mathrm{DA}}$ are known. The resistivity $\rho$ can be explicitly expressed in the following form

$$
\rho=\frac{\pi d}{\ln 2} \frac{R_{\mathrm{AB}, \mathrm{CD}}+R_{\mathrm{BC}, \mathrm{DA}}}{2} f .
$$

Here, $\mathrm{f}$ is a function of the ratio $R_{\mathrm{AB}, \mathrm{CD}} / R_{\mathrm{BC}, \mathrm{DA}}$ and can take a value between 0 and 1 (see Table A.1 $\sqrt{26 / 27 / 29}$ ). Large values of this ratio are undesirable and can suggest that either the contacts are not suitable or that the sample is inhomogeneously doped 78 .

\begin{tabular}{cc}
\hline$\frac{R_{\mathrm{AB}, \mathrm{CD}}}{R_{\mathrm{BC}, \mathrm{DA}}}$ & $\mathrm{f}$ \\
\hline \hline 1 & 1.00 \\
5 & 0.82 \\
10 & 0.70 \\
20 & 0.59 \\
100 & 0.40 \\
1000 & 0.26 \\
\hline
\end{tabular}

TABle A.1: Approximate values of the function $\mathrm{f}$, which is required to measure the resistivity of a sample of arbitrary shape via the van der Pauw method $26|27| 29$.

The measurement of the resistivity is simplified when $\mathrm{f}$ is equal to 1 , therefore $R_{\mathrm{AB}, \mathrm{CD}}=$ $R_{\mathrm{BC}, \mathrm{DA}}$. This is the case when the sample in question has a line of symmetry with contacts A and C placed at either end while contacts B and D are placed symmetrically either side of this line (see Figure A.1(b)). In this case only one resistance measurement is required to determine the resistivity of the sample, Equation A.4 is reduced to the following

$$
\rho=\frac{\pi d}{\ln 2} R_{\mathrm{AB}, \mathrm{CD}}
$$

For a circular disk, the four contacts (A, B, C and D) should be placed at the corners of a square (equidistant around the disk's perimeter) as illustrated in Figure A.2. 


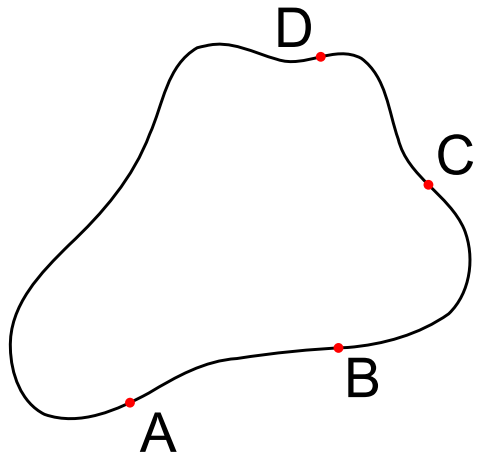

(a)

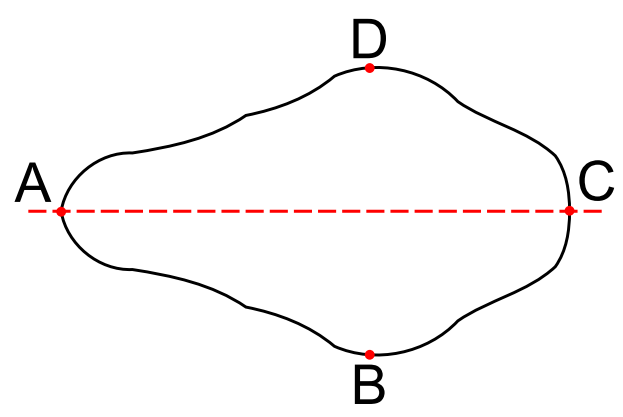

(b)

Figure A.1: (a) An arbitrary shaped disk: the van der Pauw method requires two resistance measurements in order to determine the sheet resistivity. (b) A disk containing a line of symmetry: this simplification allows the sheet resistivity to be obtained with only one resistance measurement 26 26.

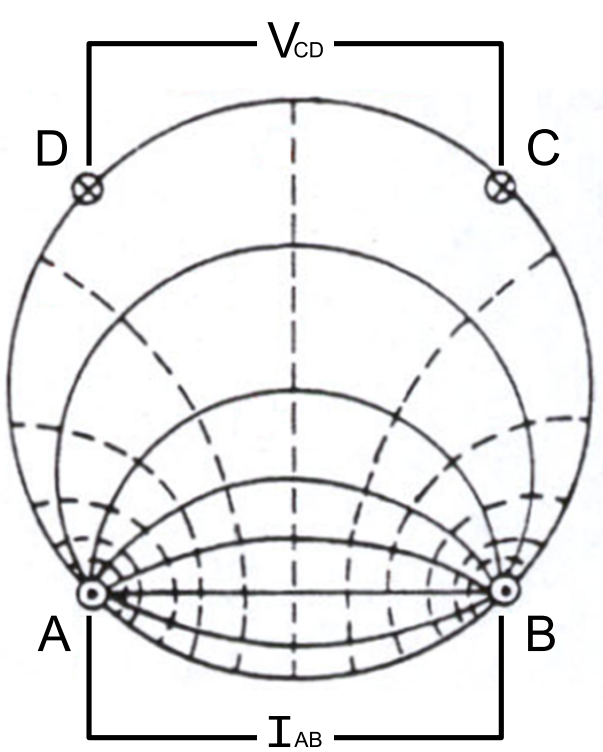

Figure A.2: The van der Pauw method for a circular disk can be used to determine the resistivity of the material with the need for only one resistance measurement. Here, the current streamlines (continuous lines) and equipotentials (dashed lines) in the system are presented. (Adapted from reference 28). 
Experimentally, the point contacts will not be perfect and so will produce associated errors in the measurement of the resistivity. These errors can be estimated, three specific cases of non-ideal contacts were considered by van der Pauw 27 . Figure A.3 demonstrates the three situations, which are:

- (a) One contact has length 1 along the circumference;

- (b) One contact has length 1 perpendicular to the circumference; and

- (c) One contact, although a point contact, is situated a distance 1 from the circumference.

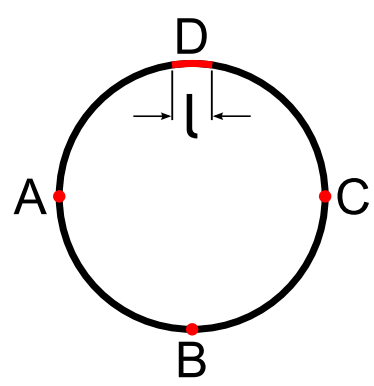

(a)

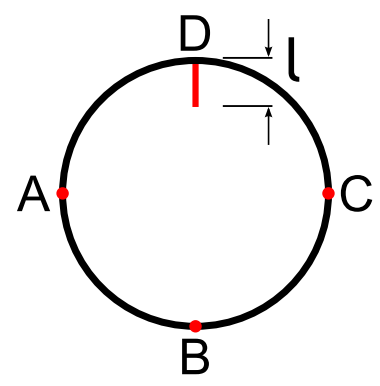

(b)

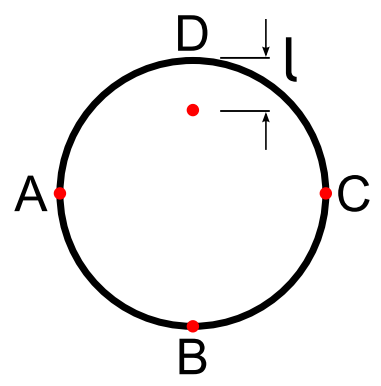

(c)

Figure A.3: The three cases of non-ideal electrical contacts where the resultant errors were considered by van der Pauw 27 .

The estimates of the errors for these three cases are summarised in Table A.2 ${ }^{26}$. In these expressions, $\varphi$ is the diameter of the van der Pauw disk that is being considered. In practice none of the contacts will be ideal and therefore, to the first approximation, the total error is the sum of the errors per contact ${ }^{27}$.

\begin{tabular}{cc}
\hline case & $\frac{\frac{\Delta \rho}{\rho}}{\mathrm{a}} \approx \frac{-l^{2}}{16 \varphi^{2} \ln 2}$ \\
\hline \hline $\mathrm{b}$ & $\approx \frac{-l^{2}}{4 \varphi^{2} \ln 2}$ \\
\hline $\mathrm{c}$ & $\approx \frac{-l^{2}}{2 \varphi^{2} \ln 2}$ \\
\hline
\end{tabular}

TABLE A.2: Summary of the error in the resistivity originating from three specific cases of non-ideal contacts within the van der Pauw method 27 . 


\section{Appendix B}

\section{Conformal Mapping}

Conformal mapping is a mathematical technique that maps points from one complex plane onto another. This type of transformation preserves the local angles between the two planes. This technique has been utilised in order to map an EMR device with a circular geometry (internal metallic shunt) into a simpler linear (rectangular) geometry (external metallic shunt) ${ }^{76}$. A simplistic interpretation of this transformation is to think about making a cut along the radius of the circular geometry device and 'unrolling' it into a linear device. The nature of conformal mapping means the two devices should be electrically identical. The production of a linear EMR device is advantageous for practical applications since the fabrication techniques for such a device are simpler and therefore more cost-effective. The specific conformal mapping used in this case is the bilinear transformation

$$
z(t)=-i \frac{t+i}{t-i}
$$

Here, $\mathrm{z}=\mathrm{x}+$ iy and $\mathrm{t}=\mathrm{r}+$ is with the complex conjugates $\bar{z}=\mathrm{x}-$ iy and $\bar{t}=\mathrm{r}-$ is respectively. In this section the bilinear transformation is applied to a circular EMR geometry in order to produce a linear EMR device*. The real and imaginary parts of $\mathrm{z}$ can be expressed as follows

$$
\begin{aligned}
& \operatorname{Re}(z)=\frac{1}{2}(z+\bar{z}), \\
& \operatorname{Im}(z)=\frac{1}{2 i}(z-\bar{z}) .
\end{aligned}
$$

\footnotetext{
${ }^{*}$ The analysis here adheres to that of a similar device as presented in the book Hall Effect Devices ${ }^{76}$
} 

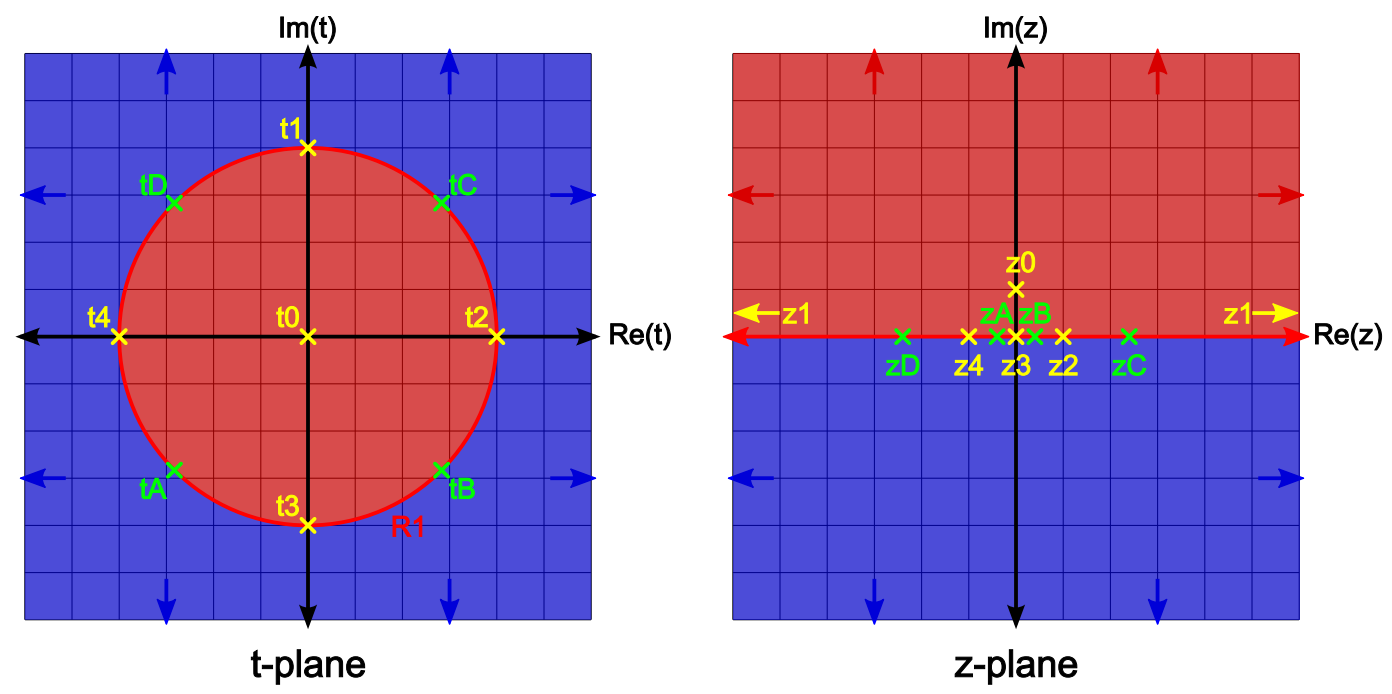

FiguRE B.1: Mapping of a unit circle (R1) centred at the origin and various points of interest from the complex t-plane to the complex z-plane with the use of the bilinear transformation. Points inside the circle correspond to points in the positive half of the complex z-plane.

Initially, a circle of unit radius centred at the origin of the t-plane is mapped onto the z-plane. In order to do this we consider the real and imaginary components of $\mathrm{z}$ by substituting Equation B.1 into Equation B.2 and B.3 respectively

$$
\begin{aligned}
& \operatorname{Re}(z)=\frac{t+\bar{t}}{t \bar{t}+i t-i \bar{t}+1}, \\
& \operatorname{Im}(z)=\frac{1-t \bar{t}}{t \bar{t}+i t-i \bar{t}+1} .
\end{aligned}
$$

Since, $t \bar{t}=|t|^{2}=r^{2}+s^{2}$ these expressions become

$$
\begin{aligned}
& \operatorname{Re}(z)=\frac{2 r}{r^{2}+s^{2}-2 s+1}, \\
& \operatorname{Im}(z)=\frac{1-r^{2}-s^{2}}{r^{2}+s^{2}-2 s+1} .
\end{aligned}
$$

For a unit circle centred at the origin $t \bar{t}=1$, this can be used to reduce the real and imaginary parts of $\mathrm{z}$

$$
\operatorname{Re}(z)=x=\frac{r}{1-s}
$$




$$
\operatorname{Im}(z)=y=0
$$

All the points on the unit circle in the t-plane, map onto the real axis of the z-plane. For points where $t \bar{t}<1$, the imaginary part of $\mathrm{z}$ (given in Equation B.7) is positive. This is because $|s|<t \bar{t}<1$ for points inside the unit circle. Therefore a unit circle centred at the origin in the t-plane, maps onto the positive imaginary axis in the z-plane (see Figure B.1). Certain points of interest on the t-plane can be mapped onto the z-plane. Using Equations B.1, B.6, B.7, B.8 and B.9 we can find the corresponding points from the t-plane in the z-plane. Table B.1 shows the real and imaginary parts of the points in the $t$ and z-planes.

\begin{tabular}{cccccc}
\hline $\begin{array}{c}\text { Point on } \\
\text { t-plane }\end{array}$ & $\operatorname{Re}(\mathrm{t})$ & $\operatorname{Im}(\mathrm{t})$ & $\begin{array}{c}\text { Point on } \\
\text { z-plane }\end{array}$ & $\operatorname{Re}(\mathrm{z})$ & $\operatorname{Im}(\mathrm{z})$ \\
\hline $\mathrm{t} 0$ & 0 & 0 & $\mathrm{z} 0$ & 0 & 1 \\
$\mathrm{t} 1$ & 0 & 1 & $\mathrm{z} 1$ & $\infty$ & 0 \\
$\mathrm{t} 2$ & 1 & 0 & $\mathrm{z} 2$ & 1 & 0 \\
$\mathrm{t} 3$ & 0 & -1 & $\mathrm{z} 3$ & 0 & 0 \\
$\mathrm{t} 4$ & -1 & 0 & $\mathrm{z} 4$ & -1 & 0 \\
$\mathrm{tA}$ & $\frac{-1}{\sqrt{2}}$ & $\frac{-1}{\sqrt{2}}$ & $\mathrm{zA}$ & $\frac{-1}{1+\sqrt{2}}$ & 0 \\
$\mathrm{tB}$ & $\frac{1}{\sqrt{2}}$ & $\frac{-1}{\sqrt{2}}$ & $\mathrm{zB}$ & $\frac{1}{1+\sqrt{2}}$ & 0 \\
$\mathrm{tC}$ & $\frac{1}{\sqrt{2}}$ & $\frac{1}{\sqrt{2}}$ & $\mathrm{zC}$ & $\frac{1}{-1+\sqrt{2}}$ & 0 \\
$\mathrm{tD}$ & $\frac{-1}{\sqrt{2}}$ & $\frac{1}{\sqrt{2}}$ & $\mathrm{zD}$ & $\frac{-1}{-1+\sqrt{2}}$ & 0 \\
\hline
\end{tabular}

TABLE B.1: The real and imaginary components of corresponding points in the $t$ and z-planes as seen in Figure B.1

This simple unit circle produces a linear device that is infinite in the positive imaginary $\mathrm{z}$ axis and both the positive and negative real $\mathrm{z}$ axis. In order to produce a finite sized linear device some cuts are required to be made to the circular device in the t-plane. In practice a line is introduced in the z-plane in order to bound the device and this line is then mapped back onto the t-plane. A horizontal line at $\operatorname{Im}(z)=y 2$ is introduced in the z-plane in order to eliminate all values of $\mathrm{z}$ with an imaginary part greater than $\mathrm{y} 2$. The introduction of this line and the corresponding cut to the device in the t-plane can be seen in Figure B.2.

In order to map this horizontal line back to the t-plane we set Equation B.7 equal to y2 and rearrange. This results in the formula for a circle in the t-plane

$$
r^{2}+\left(s-\frac{y 2}{y 2+1}\right)^{2}=\frac{1}{(y 2+1)^{2}} .
$$



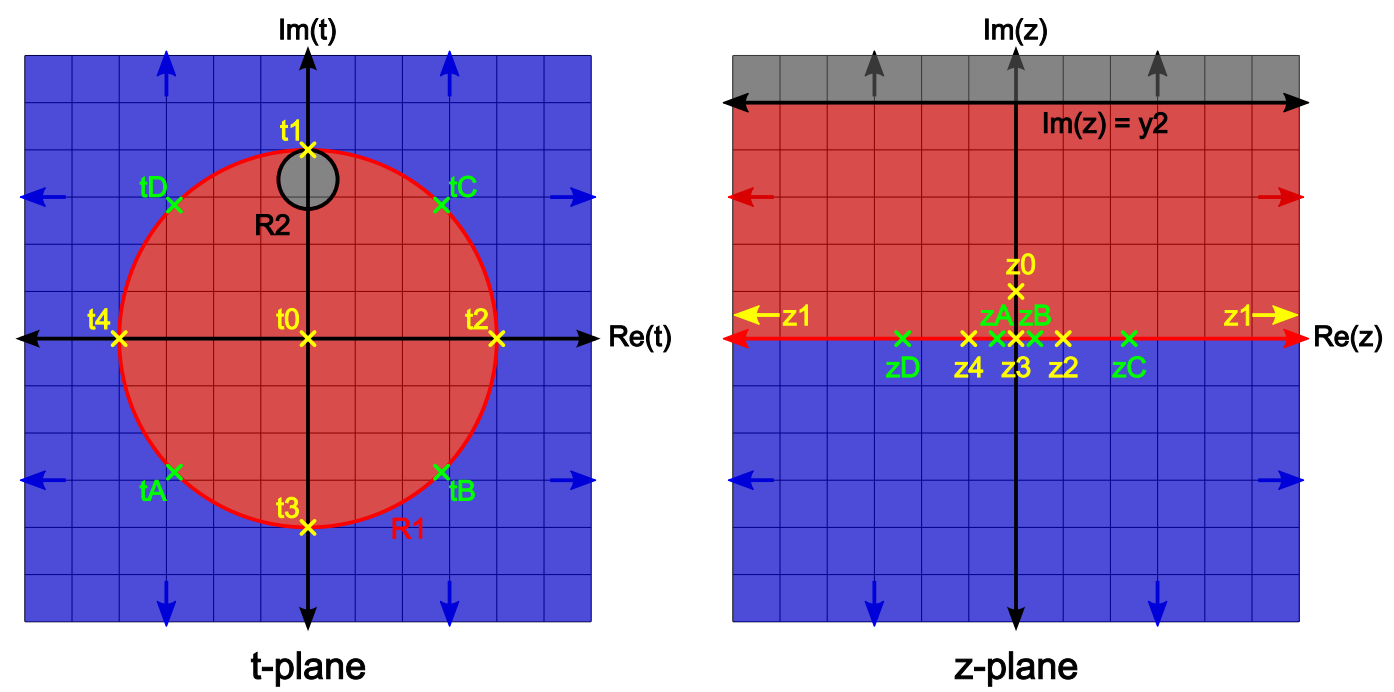

Figure B.2: The addition of a line with $\operatorname{Im}(\mathrm{z})=\mathrm{y} 2$ in the $\mathrm{z}$-plane eliminates all values of $\mathrm{z}$ with $\operatorname{Im}(\mathrm{z})>\mathrm{y} 2$ thus making the device finite in the $\operatorname{Im}(\mathrm{z})$ direction. This line corresponds to a circle in the t-plane (R2).

The radius of this circle is $R 2=\frac{1}{y^{2+1}}$ and is centred at the point $\left(0, \frac{y 2}{y^{2}+1}\right)$ in the t-plane. To produce a linear device with values of $z$ that have finite real components, a vertical line is introduced in the $\mathrm{z}$-plane $(\operatorname{Re}(\mathrm{z})=\mathrm{x} 3)$ to exclude all values of $\mathrm{z}$ with a real part greater than $x 3$. This is done by setting Equation B.6 equal to $x 3$ and rearranging. This produces the formula for another circle in the t-plane

$$
\left(r-\frac{1}{x 3}\right)^{2}+(s-1)^{2}=\frac{1}{x 3^{2}}
$$

The radius of this circle is $R 3=\frac{1}{x^{3}}$ and is centred at the point $\left(\frac{1}{x^{3}}, 1\right)$ in the t-plane.

By introducing a similar vertical line at $\operatorname{Re}(\mathrm{z})=\mathrm{x} 4$ all values of $\mathrm{z}$ with a real part less than $\mathrm{x} 4$ are eliminated from the linear device in the $\mathrm{z}$-plane, resulting in a finite device. This line at $\operatorname{Re}(\mathrm{z})=\mathrm{x} 4$ produces another circle in the t-plane

$$
\left(r-\frac{1}{x 4}\right)^{2}+(s-1)^{2}=\frac{1}{x 4^{2}}
$$

The radius of this circle is $R 4=\frac{1}{x 4}$ and is centred at the point $\left(\frac{1}{x 4}, 1\right)$ in the t-plane.

The introduction of these two vertical lines in the z-plane and the corresponding circles in the t-plane can be seen in Figure B.3.

Finally, a second region (representing the metallic disk) can be created in the linear device by introducing a horizontal line at $\operatorname{Im}(\mathrm{z})=\mathrm{y} 5$ in the z-plane. This results in the 

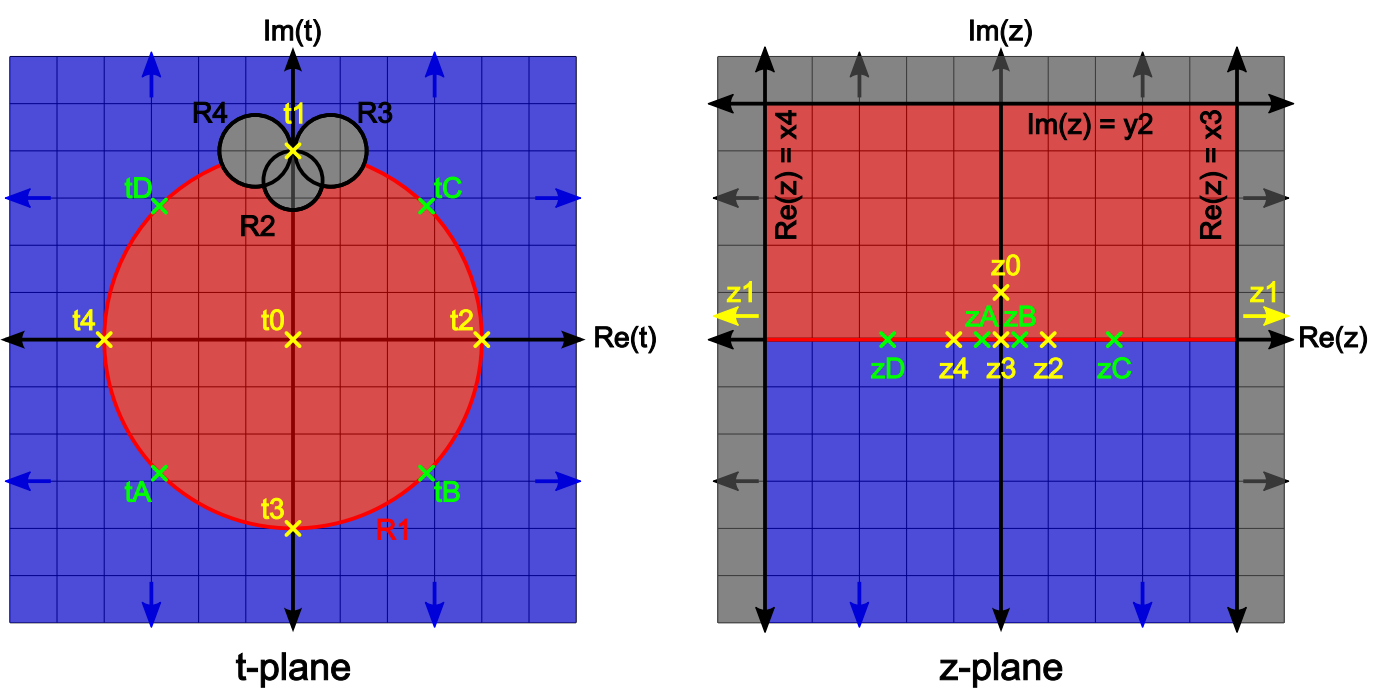

Figure B.3: The addition of two vertical lines in the $z-p l a n e(\operatorname{Re}(z)=x 3$ and $\operatorname{Re}(z)=x 4)$ eliminate all values of $\mathrm{z}$ with $\operatorname{Re}(\mathrm{z})>\mathrm{x} 3$ and $\operatorname{Re}(\mathrm{z})<\mathrm{x} 4$, producing a finite sized rectangular device in the z-plane. These two lines correspond to two circles in the t-plane (R3 and $\mathrm{R} 4)$.

introduction of a circle in the t-plane (of the same form as previously seen when the line $\operatorname{Im}(\mathrm{z})=\mathrm{y} 2$ was introduced in the z-plane)

$$
r^{2}+\left(s-\frac{y 5}{y 5+1}\right)^{2}=\frac{1}{(y 5+1)^{2}} .
$$

The radius of this circle is $R 5=\frac{1}{y 5+1}$ and is centred at the point $\left(0, \frac{y 5}{y 5+1}\right)$ in the t-plane. The region inside this circle in the t-plane corresponds to the rectangle bounded by the lines $\operatorname{Im}(\mathrm{z})=\mathrm{y} 2, \operatorname{Im}(\mathrm{z})=\mathrm{y} 5, \operatorname{Re}(\mathrm{z})=\mathrm{x} 3$ and $\operatorname{Re}(\mathrm{z})=\mathrm{x} 4$ in the $\mathrm{z}$-plane.

The final device produced can be seen in Figure B.4. Here, the bilinear transformation has been used to create a linear geometry EMR device from a circular geometry device. There are some differences between the circular device, used initially by Solin et al. when the EMR effect was discovered, and the one presented here that maps to the linear device. In this section we have a circular geometry device with an off-centre metallic region (seen in green in Figure B.4 with three small circular cuts removed in order to produce a finite sized linear device. Also, the contacts tA, tB, tC and tD that are equidistantly placed around the circumference of the circular device in the t-plane correspond to points that are not equally distributed along the real axis in the z-plane. Finally, a doubling of the filling fraction in the circular device in the t-plane does not lead to a doubling of the filling fraction of the linear device in the z-plane. As a result of these factors there will be some discrepancy between the resultant EMR effect produced from the circular device geometry of Solin et al., and a device based on the linear geometry produced here. 


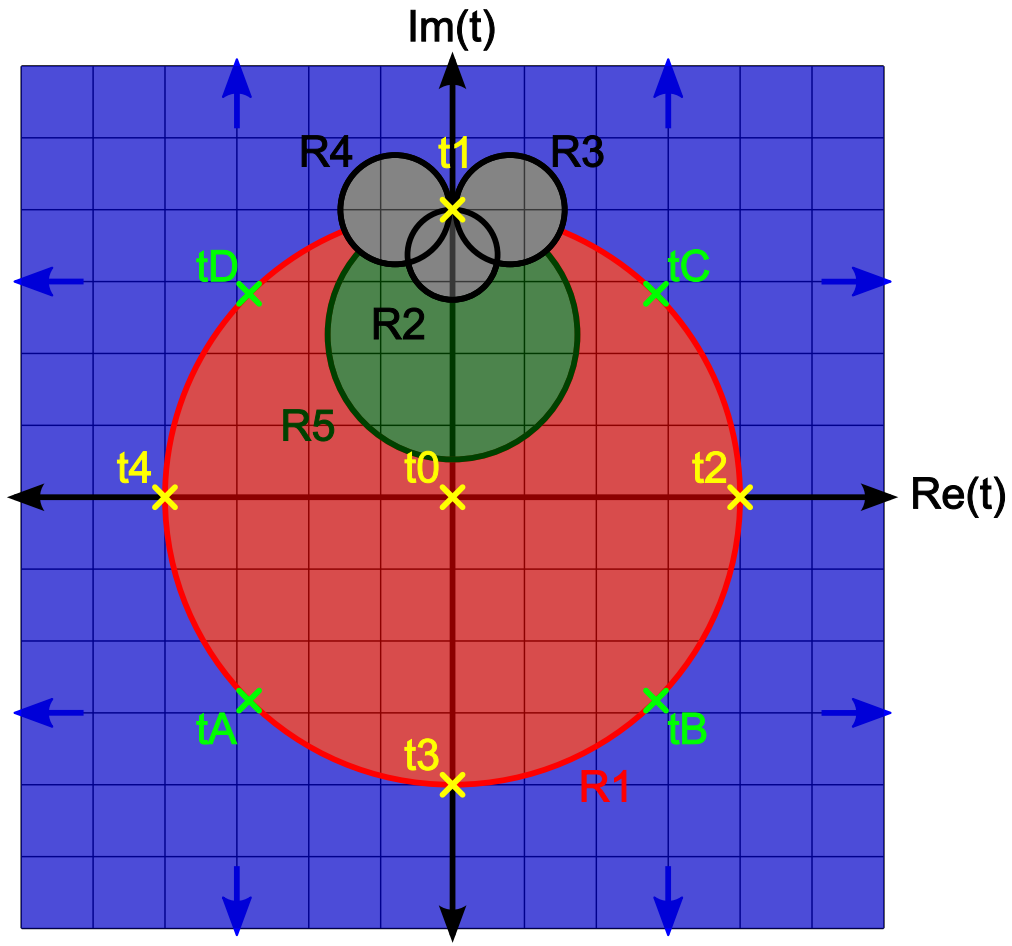

\section{t-plane}

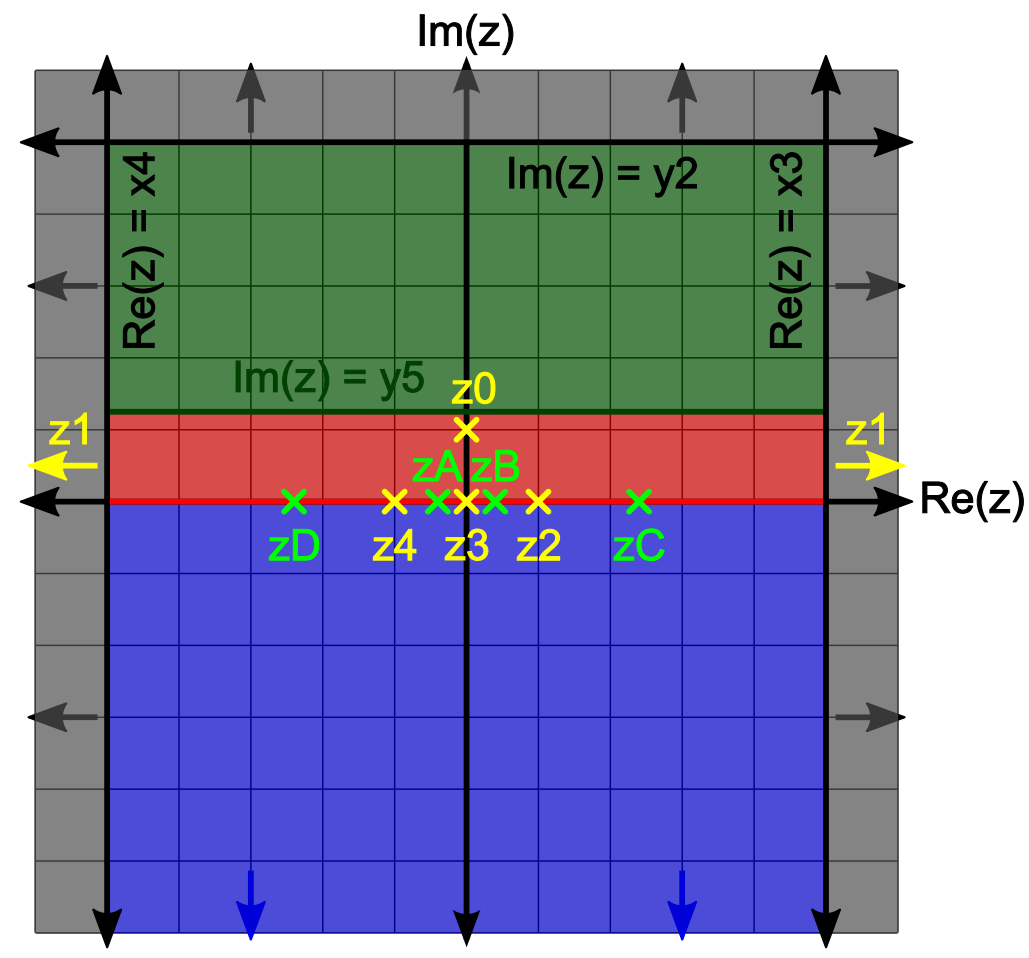

\section{z-plane}

FiguRE B.4: Introducing a second horizontal line in the z-plane at $\operatorname{Im}(\mathrm{z})=\mathrm{y} 5$ produces a rectangular device with two separate regions. This line corresponds to the circle R5 in the t-plane and thus showing how a circular geometry can be mapped into a linear rectangular one. 


\section{Appendix $\mathrm{C}$}

\section{Research Poster}

The following research poster (along with similar ones) has been exhibited at various conferences, summer schools and poster competitions between 2009 and 2011 (see list below). It was designed in order to try and convey the concept of extraordinary magnetoresistance to a non-subject specialist audience.

\section{Poster Presentations}

- TeraMat09, Benasque, Spain, December 2009.

- Graduate School Poster Competition, Loughborough University, UK, May 2010.

- Physics by the Lake Summer School, University of Cumbria, UK, July 2010.

- Science Poster Competition, Loughborough University, UK, April 2011. (Awarded Commendation Prize)

- NES 2011, Bath, UK, May 2011.

- SigmaPhi 2011, Larnaca, Cyprus, July 2011. 


\section{Extraordinary Magnetoresistance: Sensing The Future}

Magnetoresistance is a change in electrical resistance due to a magnetic field. Extraordinary Magnetoresistance (EMR) is a new form of this effect discovered in 2000 . We propose a new system that increases the magnetoresistance by a factor of 10,000 . EMR has a huge number of potential uses in the sensing of magnetic fields, especially in producing future hard disks.

1. Potential Applications of EMR

- The EMR effect could lead to improved magnetic field sensors with higher sensitivity, and the possibility of developing faster and higher capacity computer hard disks.

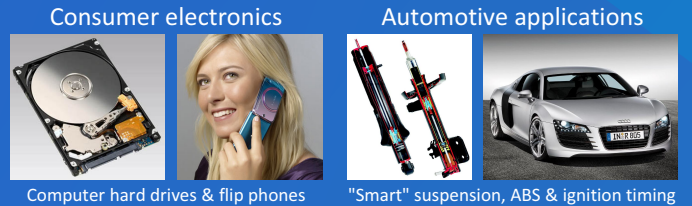

2. Experimental Discovery

- EMR was discovered in systems where a metallic disk was embedded in a larger semiconducting disk. Many types of magnetoresistance are known, with EMR producing the largest change in resistance of them all.
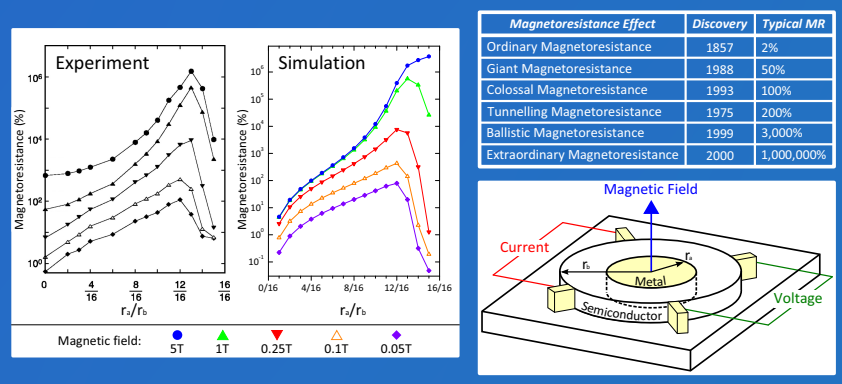

\section{Results and Modelling}

- We have shown that the EMR effect strongly depends on the changing the shape of the metallic region

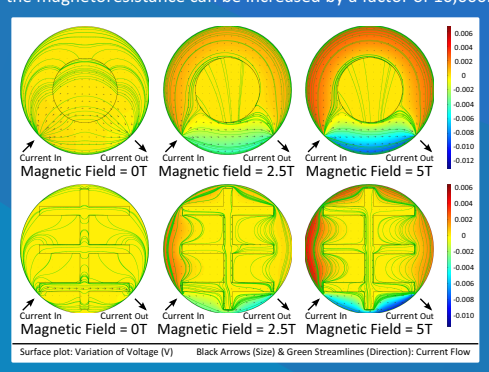

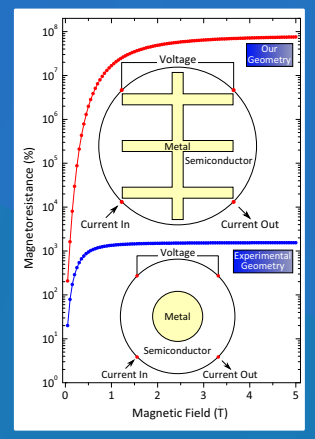

Medical applications

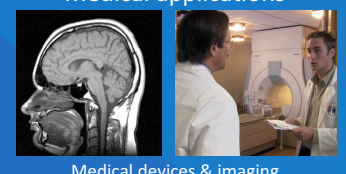

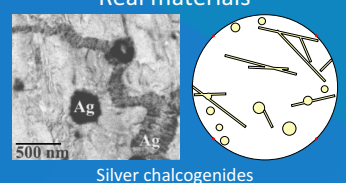

3. The Mechanism Behind EMR

- The electrical resistance of a semiconductor is over 2000 times larger than that of a metal.

- Consider a semiconductor containing a region of metal, apply a voltage across the system and the majority of the current will flow through the metal
(low resistance path) when there is no magnetic field.

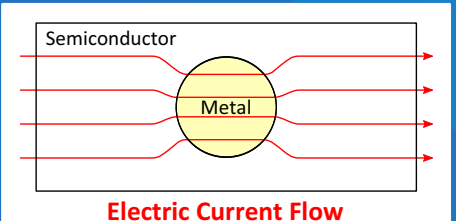

Electric Current Flow

- Electric field lines align at right angles to a metallic surface and with no magnetic field the current flows along these lines into the metal.

- However, when a magnetic field is applied to the system the current is forced to flow at right angles to the metallic surface.

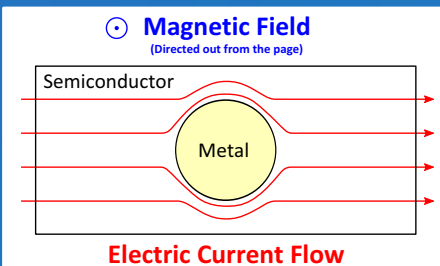

Electric Current Flow

- This forces the current to flow away from the metal and through the semiconductor along a path of much higher resistance.

We have verified existing experimental EMR results and found a new shape that strongly influences the magnetoresistance. With magnetic field sensors now an everyday part of modern life, a vast number of improvements could be realised with this new technology.

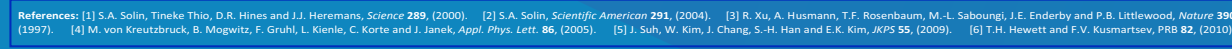

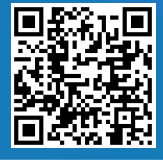

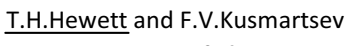
Department of Physics
Contact Details: T.H.Hewett@lboro.ac.uk
Loughborough University 


\section{Appendix D}

\section{Comsol Multiphysics Code}

The following code was implemented for the simulation of magnetoresistance of systems containing a circular conducting region (as for the results seen in Chapter 3). The code can be executed as an m-file by COMSOL Multiphysics Version 3.4 (requires Comsol script functionality). In order to produce a different sized metallic droplet the value of $\mathrm{n}$ can be varied (between 1 and 15). This code is the basis of other more complex systems that require manipulation of the geometric parameters. The material parameters included here are those found in the experimental systems of Solin et al. when the EMR effect was discovered (see Table 3.1).

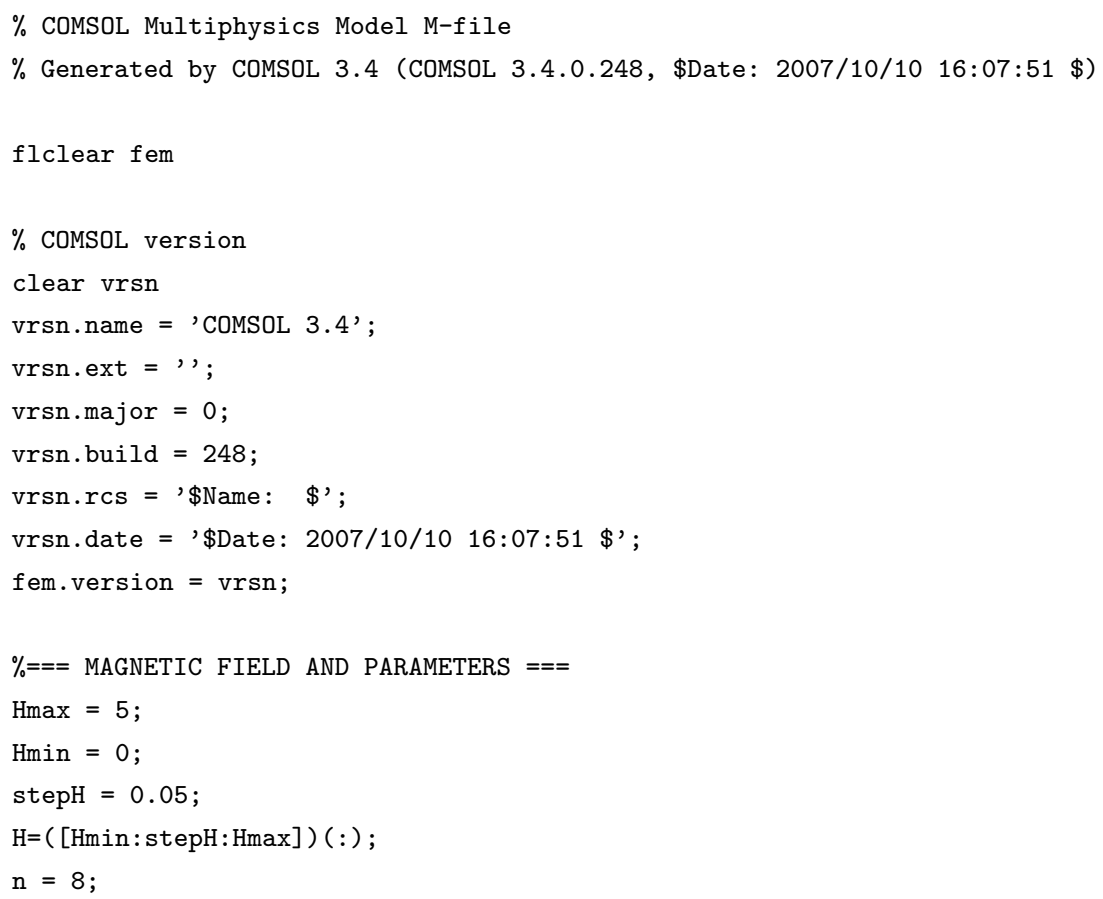




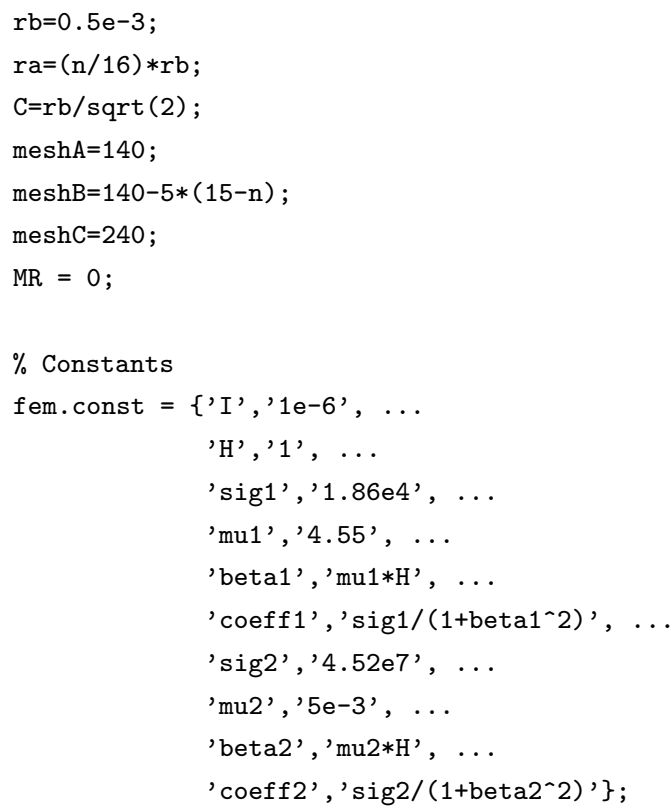

\section{$\%$ Geometry}

g1=circ2 (num2str(rb) , 'base', 'center', 'pos', \{'0', '0'\}, 'rot' , '0') ;

g2=circ2 (num2str(ra), 'base', 'center', 'pos', $\{$ '0', '0'\}, 'rot', '0') ;

parr $=\{$ point $2(C, C)\}$;

g3=geomcoerce ('point', parr);

parr $=\{$ point $2(-\mathrm{C}, \mathrm{C})\}$;

g4=geomcoerce ('point', parr);

parr $=\{$ point $2(-\mathrm{C},-\mathrm{C})\}$;

g5=geomcoerce ('point', parr);

parr $=\{$ point $2(\mathrm{C},-\mathrm{C})\}$;

g6=geomcoerce ('point', parr);

$\%$ Analyzed geometry

clear p s

$\mathrm{p} . \mathrm{objs}=\{\mathrm{g} 3, \mathrm{~g} 4, \mathrm{~g} 5, \mathrm{~g} 6\}$;

p. name $=\left\{\right.$ 'C' $^{\prime}, D^{\prime}$, , $^{\prime}$ ' , 'B' $\}$;

p.tags $=\left\{{ }^{\prime} g 3^{\prime},,^{\prime} g 4^{\prime},,^{\prime} g 5^{\prime},{ }^{\prime} g 6^{\prime}\right\}$;

s. objs $=\{\mathrm{g} 1, \mathrm{~g} 2\}$

s. name $=\{$ 'Semiconductor', 'Metal' $\}$;

s.tags $=\{$ 'g1', 'g2'\};

fem.draw=struct ('p',p, 's', s);

fem. geom=geomcsg (fem);

fem.mesh=meshinit $(\mathrm{fem}, \ldots$

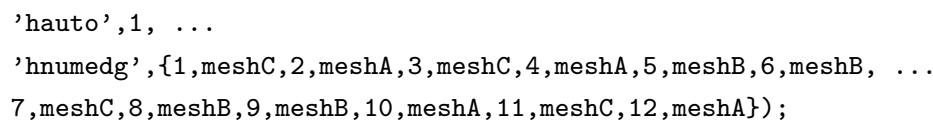




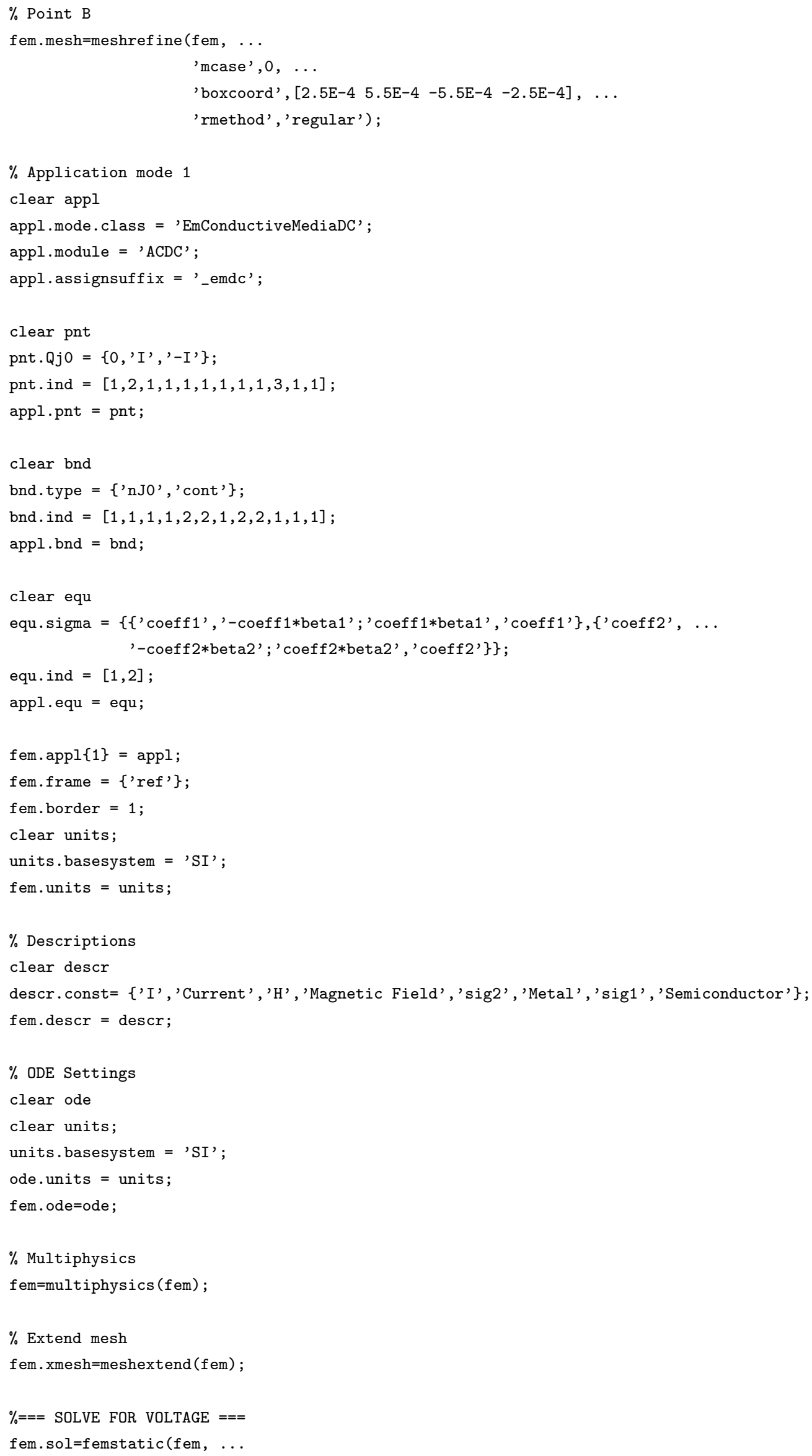




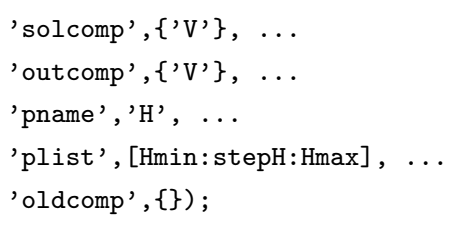

$\%$ Save current fem structure for restart purposes

$f e m 0=f e m$;

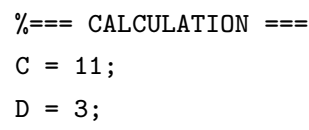




\section{Bibliography}

[1] J. De Launay, R. L. Dolecek, and R. T. WebBer, Journal of Physics and Chemistry of Solids 11, 37 (1959).

[2] J. Nickel, Magnetoresistance Overview, Hewlett-Packard Technical Report HPL-95-60, 1995

[3] M. N. Baibich, J. M. Broto, A. Fert, F. N. Van Dau, F. Petroff, P. Etienne, G. Creuzet, A. Friederich, and J. Chazelas, Physical Review Letters 61, 2472 (1988).

[4] G. XIaO, What is Spintronics, Brown University, 2011

[5] M. Walter, Magnetic Tunneling Junction (MTJ), University of Konstanz Technical Report, 2009.

[6] P. Schiffer, A. P. Ramirez, W. Bao, and S.-W. Cheong, Physical Review Letters 75, 3336 (1995).

[7] N. García, M. Muñoz, V. V. Osipov, E. V. Ponizovskaya, G. G. Qian, I. G. Saveliev, and Y.-W. ZHAO, Journal of Magnetism and Magnetic Materials 240, 92 (2002).

[8] J. Heremans, Journal of Physics D: Applied Physics 26, 1149 (1993).

[9] S. A. Solin, T. Thio, D. R. Hines, and J. J. Heremans, Science 289, 1530 (2000).

[10] J. Suh, W. Kim, J. Chang, S.-H. Han, and E. K. KIm, Journal of the Korean Physical Society 55, 577 (2009).

[11] A. L. Friedman, J. T. Robinson, F. K. Perkins, and P. M. Campbell, Applied Physics Letters 99, $022108(2011)$.

[12] J. Lu, H. Zhang, W. Shi, Z. Wang, Y. Zheng, T. Zhang, N. Wang, Z. Tang, and P. Sheng, Nano Letters 11, 2973 (2011).

[13] J. Moussa, L. R. Ram-Mohan, J. Sullivan, T. Zhou, D. R. Hines, and S. A. Solin, Physical Review B 64, $184410(2001)$.

[14] S. A. Solin, Scientific American 291, 45 (2004).

[15] S. A. Solin, ArXiv Condensed Matter e-prints (2006).

[16] M. Holz, O. Kronenwerth, and D. Grundler, Applied Physics Letters 83, 3344 (2003).

[17] M. Holz, The Extraordinary Magnetoresistance of Semiconductor-Metal Hybrid Structures: Modeling and Optimization, Cuvillier Verlag, 2004

[18] M. Holz, O. Kronenwerth, and D. Grundler, Applied Physics Letters 86, 072513 (2005).

[19] M. Holz, O. Kronenwerth, and D. Grundler, Physical Review B 67, 195312 (2003).

[20] C.-B. Rong, H.-W. Zhang, J.-R. Sun, and B.-G. Shen, Journal of Magnetism and Magnetic Materials 301, 407 (2006). 
[21] J. Sun and J. Kosel, Journal of Superconductivity and Novel Magnetism, DOI: 10.1007/s10948-011-1256-8 (2011).

[22] R. Xu, A. Husmann, T. F. Rosenbaum, M.-L. Saboungi, J. E. Enderby, and P. B. Littlewood, Nature 390, 57 (1997).

[23] A. Husmann, J. B. Betts, G. S. Boebinger, A. Migliori, T. F. Rosenbaum, and M.-L. Saboungi, Nature 417, 421 (2002).

[24] M. von Kreutzbruck, B. Mogwitz, F. Gruhl, L. Kienle, C. Korte, and J. Janek, Applied Physics Letters 86, $072102(2005)$.

[25] M. M. Parish and P. B. Littlewood, Nature 426, 162 (2003).

[26] L. J. VAn Der PAuw, Philips Research Reports 13 (1958).

[27] L. J. VAn Der Pauw, Philips Technical Review 20, 220 (1958).

[28] M. Shur, GaAs Devices and Circuits, Plenum Press, 1986

[29] K. Seeger, Semiconductor Physics, Springer Verlag, 2004

[30] C. Kittel, Quantum Theory of Solids, Wiley, 1963

[31] W. Thompson, Proceedings of the Royal Society of London 8, 546 (1857).

[32] A. B. PipPard, Magnetoresistance in Metals, Cambridge University Press, 1989.

[33] B. Mogwitz, C. Korte, J. Janek, M. von Kreutzbruck, and L. Kienle, Journal of Applied Physics 101, 043510 2007).

[34] M. von Kreutzbruck, G. Lembke, B. Mogwitz, C. Korte, and J. Janek, Physical Review B 79, 035204 (2009).

[35] A. A. Abrikosov, Europhysics Letters 49, 789 (2000).

[36] A. A. Abrikosov, Journal of Physics A: Mathematical and General 36, 9119 (2003).

[37] A. A. Abrikosov, Physical Review B 58, 2788 (1998.

[38] M. M. Parish and P. B. Littlewood, Physical Review B 72, 094417 (2005).

[39] J. Hu, M. M. Parish, and T. F. Rosenbaum, Physical Review B 75, 214203 (2007).

[40] S. A. Bulgadaev and F. V. Kusmartsev, Physics Letters A 337, 449 (2005).

[41] S. A. Bulgadaev and F. V. Kusmartsev, Physics Letters A 342, 188 (2005).

[42] S. A. Bulgadaev, Physics Letters A 344, 280 (2005).

[43] S. A. Bulgadaev and F. V. Kusmartsev, Physics Letters A 336, 223 (2005).

[44] J. Xu, D. Zhang, F. Yang, Z. Li, Z. Deng, and Y. Pan, Journal of Physics D: Applied Physics 41, 115003 (2008).

[45] J. Xu and D. Zhang, Communications in Theoretical Physics 55, 532 (2011).

[46] J. Xu, D. Zhang, Z. Deng, F. Yang, Z. LI, and Y. Pan, Chinese Physics Letters 25, 4124 (2008).

[47] J. Xu, D. Zhang, F. Yang, Z. LI, and Y. Pan, Journal of Applied Physics 104, 113922 (2008).

[48] V. Guttal and D. Stroud, Physical Review B 73, 085202 (2006). 
[49] D. Stroud, Physical Review B 12, 3368 (1975).

[50] D. J. Bergman and Y. M. Strelniker, Physical Review B 49, 16256 (1994).

[51] D. J. Bergman and Y. M. Strelniker, Physical Review B 59, 2180 (1999).

[52] D. J. Bergman and Y. M. Strelniker, Physical Review B 60, 13016 (1999).

[53] R. Magier and D. J. Bergman, Physical Review B 74, 094423 (2006).

[54] G. T. Meaden, Electrical Resistance of Metals, Heywood Books, 1966

[55] A. W. Rushforth, K. Výborný, C. S. King, K. W. Edmonds, R. P. Campion, C. T. Foxon, J. Wunderlich, A. C. Irvine, P. Vašek, V. Novák, K. Olejník, J. Sinova, T. Jungwirth, and B. L. Gallagher, Physical Review Letters 99, 147207 (2007).

[56] G. Binasch, P. Grünberg, F. Saurenbach, and W. Zinn, Physical Review B 39, 4828 (1989).

[57] S. Ikeda, J. Hayakawa, Y. Ashizawa, Y. M. Lee, K. Miura, H. Hasegawa, M. Tsunoda, F. Matsukura, and H. Ohno, Applied Physics Letters 93, 082508 (2008).

[58] M. Julliere, Physics Letters A 54, 225 (1975).

[59] A. P. Ramirez, Journal of Physics: Condensed Matter 9, 8171 (1997).

[60] G. H. Jonker and J. H. van Santen, Physica 16, 337 (1950).

[61] S. Jin, T. H. Tiefel, M. McCormack, R. A. Fastnacht, R. Ramesh, and L. H. Chen, Science 264, 413 (1994).

[62] C. Zener, Physical Review 82, 403 (1951).

[63] N. García, M. Muñoz, and Y.-W. ZhaO, Physical Review Letters 82, 2923 (1999).

[64] S. Z. Hua and H. D. Chopra, Physical Review B 67, 060401 (2003).

[65] H. D. Chopra and S. Z. Hua, Physical Review B 66, 020403 2002.

[66] B. Doudin and M. Viret, Journal of Physics: Condensed Matter 20, 083201 (2008).

[67] W. F. Egelhoff Jr., L. Gan, H. Ettedgui, Y. Kadmon, C. J. Powell, P. J. Chen, A. J. Shapiro, R. D. McMichael, J. J. Mallett, T. P. Moffat, M. D. Stiles, and E. B. Svedberg, Journal of Magnetism and Magnetic Materials 287, 496 (2005).

[68] W. F. Egelhoff Jr., L. Gan, H. Ettedgui, Y. Kadmon, C. J. Powell, P. J. Chen, A. J. Shapiro, R. D. McMichael, J. J. Mallett, T. P. Moffat, M. D. Stiles, and E. B. Svedberg, Journal of Applied Physics 95, 7554 (2004).

[69] J. Campbell, K. Cheung, L. Yu, J. Suehle, A. OAtes, and K. Sheng, IEEE Electron Device Letters 32 , 75 (2011).

[70] T. R. Jervis and E. F. Johnson, Solid-State Electronics 13, 181 (1970).

[71] S. A. Solin, D. R. Hines, J. S. Tsai, Y. A. Pashkin, S. J. Chung, N. Goel, and M. B. Santos, IEeE Transactions on Magnetics 38, 89 (2002).

[72] T. Zhou, D. R. Hines, and S. A. Solin, Applied Physics Letters 78, 667 (2001).

[73] S. A. Solin, D. R. Hines, A. C. H. Rowe, J. S. Tsai, Y. A. Pashin, S. J. Chung, N. Goel, and M. B. Santos, Applied Physics Letters 80, $4012(2002)$.

[74] E. H. Putley, The Hall Effect and Semiconductor Physics, Dover Publications, 1968. 
[75] N. W. Ashcroft and N. D. Mermin, Solid State Physics, Cengage Learning, 1976

[76] R. S. Popović, Hall Effect Devices, Adam Hilger, 1991

[77] T. Chakraborty and P. Pietiläinen, The Quantum Hall Effects: Fractional and Integral, Springer Verlag, 1995

[78] P. Y. Yu and M. Cardona, Fundamentals of Semiconductors, Springer Verlag, 1999.

[79] N. Miura, Physics of Semiconductors in High Magnetic Fields, Oxford University Press, 2008.

[80] J. Fish and T. Belytschko, A First Course in Finite Elements, Wiley, 2007

[81] Y. C. PAO, A First Course In Finite Element Analysis, Allyn and Bacon, 1986

[82] N. Kim and B. SANKar, Introduction to Finite Element Analysis and Design, Wiley, 2009

[83] Comsol, AC/DC Module User's Guide, Comsol Multiphysics, 2006

[84] F. S. Terra, A. A. Higazy, G. M. Mahmoud, and A. M. Mansour, Indian Journal of Physics 84, 265 (2010).

[85] C. H. Möller, O. Kronenwerth, D. Grundler, W. Hansen, C. Heyn, and D. Heitmann, Applied Physics Letters 80, 3988 (2002).

[86] M. Hoener, O. Kronenwerth, C. Heyn, D. Grundler, and M. Holz, Journal of Applied Physics 99, $036102(2006)$.

[87] A. S. Troup, D. G. Hasko, J. Wunderlich, and D. A. Williams, Applied Physics Letters 89, 022116 (2006).

[88] S. Pisana, P. M. Braganca, E. E. Marinero, and B. A. Gurney, Nano Letters 10, 341 (2010).

[89] S. Pisana, P. M. Braganca, E. E. Marinero, and B. A. Gurney, IEeE Transactions on Magnetics 46, $1910(2010)$.

[90] T. D. Boone, L. Folks, J. A. Katine, S. Maat, E. Marinero, S. Nicoletti, M. Field, G. J. Sullivan, A. IKhlassi, B. Brar, and B. A. Gurney, IEEE Transactions on Magnetics 45, 3270 (2006).

[91] J. H. Chen, C. Jang, S. Xiao, M. Ishigami, and M. S. Fuhrer, Nature Nanotechnology 3, 206 (2008).

[92] M. Holz, O. Kronenwerth, and D. Grundler, Applied Physics Letters 87, 172501 (2005).

[93] C.-B. Rong, H.-W. Zhang, J.-R. Sun, and B.-G. Shen, Applied Physics Letters 89, 052503 (2006).

[94] J. Moussa, L. R. Ram-Mohan, A. C. H. Rowe, and S. A. Solin, Journal of Applied Physics 94, 1110 (2003).

[95] F. S. Terra, A. A. Higazy, G. M. Mahmoud, and A. M. Mansour, International Journal of Nanoelectronics and Materials 3, 53 (2010).

[96] K. Rosahl, Magnetoresistance in Inhomogeneous Media, Loughborough University, 2008.

[97] M. Holz, O. Kronenwerth, and D. Grundler, Physica E 21, 897 (2004).

[98] C. H. Möller, D. Grundler, O. Kronenworth, C. Heyn, and D. Heitmann, Journal of Superconductivity 16, 195 (2003).

[99] P. Junod, Helvetica Physica Acta 32, 567 (1959).

[100] S. S. Manoharan, S. J. Prasanna, D. E. Kiwitz, and C. M. Schneider, Physical Review B 63, 212405 (2001). 
[101] I. S. Chuprakov and K. H. Dahmen, Applied Physics Letters 72, 2165 (1998).

[102] B. Q. Liang, X. Chen, Y. J. Wang, and Y. J. Tang, Physical Review B 61, 3239 (2000).

[103] G. Beck and J. Janek, Physica B 308, 1086 (2001).

[104] G. Beck, C. Korte, J. Janek, F. Gruhl, and M. von Kreutzbruck, Journal of Applied Physics 96, 5619 (2004).

[105] J. Janek, B. Mogwitz, G. Beck, M. von Kreutzbruck, L. Kienle, and C. Korte, Progress in Solid State Chemistry 32, 179 (2004).

[106] G. BeCK and J. JaneK, Solid State Sciences 10, 776 (2008).

[107] Y. Kumashiro, T. Ohachi, and I. Taniguchi, Solid State Ionics 86, 761 (1996).

[108] L. Kienle, V. Duppel, B. Mogwitz, J. Janek, M. von Kreutzbruck, A. Leineweber, and A. Simon, Crystal Growth \& Design 11, 2412 (2011).

[109] L. Kienle, V. Duppel, G. Lembke, B. Mogwitz, J. Janek, M. von Kreutzbruck, and A. Simon, Solid State Sciences 12, $1770(2010)$.

[110] W. Zhang, R. Yu, W. Feng, Y. Yao, H. Weng, X. DaI, and Z. Fang, Physical Review Letters 106, $156808(2011)$. 